\title{
Broadband, Reconfigurable and Multifunctional Elastic Wave Control with Smart Metamaterials
}

\begin{tabular}{c} 
A Dissertation \\
Presented to \\
the Faculty of the Graduate School \\
University of Missouri-Columbia \\
In Partial Fulfillment \\
of the Requirement for the Degree \\
Doctor of Philosophy \\
Pangyang Chen \\
\hline
\end{tabular}


The undersigned, appointed by the dean of Graduate School have examined the dissertation entitled

\section{Broadband, Reconfigurable and Multifunctional Elastic}

\section{Wave Control with Smart Metamaterials}

Presented by Yangyang Chen, a candidate for degree of doctor of philosophy, and hereby certify that, in their opinion, it is worthy of acceptance.

Professor Guoliang Huang

Professor Zaichun "Frank" Feng

Professor Noah D. Manring

Professor Jian Lin

Professor Zhen Chen 


\section{Acknowledgement}

I will start by thanking my supervisor, Dr. Guoliang Huang, for the opportunities and supports he has given to me during my entire $\mathrm{Ph}$. D. studies. His encouragement, patience and guidance allow me to have a pleasant and fulfilling research experience for the past five years. He trained me how to define a problem, how to solve it individually and how to prepare the academic career. As the most important mentor in my life, he taught me how to extend from a point to a surface and how to do an important and impacted research. His active and insight attitudes in academic and industrial researches and in life consistently influence me. Thank you for always being there whenever I need advice.

I would like to thank professors Zaichun "Frank" Feng, Noah D. Manring, Jian Lin and Zhen Chen for being part of my thesis committee, for patiently answering my many emails and for dedicating me some of their time.

I am grateful for the numerous discussions with all the current and former members in Dr. Huang's group, which makes my Ph. D. experience fruitful and fun. Special thanks go to Dr. Hussein Nassar, Dr. Rui Zhu and Dr. Jin Hu for their collaborations on some of the projects presented in the dissertation. I also highly appreciate the help provided by Dr. Rui Zhu, Xiaopeng Li and Miles V. Barnhart on sample fabrications and experimental testing.

The financial support from the Air Force Office of Scientific Research is also gratefully acknowledged.

Finally, I would like to thank my wife and my parents for their encourage and love during this journey. 


\section{Contents}

Acknowledgement .............................................................................................................................. ii

Abstract ................................................................................................................................ V

Chapter 1 Introduction ................................................................................................. 1

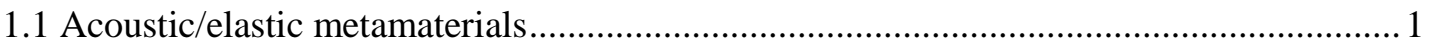

1.2 Tunable phononic crystals and metamaterials .............................................................. 4

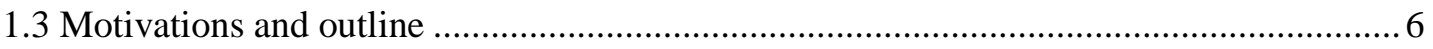

Chapter 2 An Active Elastic Metamaterial with Tunable Wave Band Gaps ........... 9

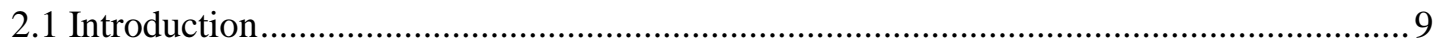

2.2 Band gap control in active elastic metamaterials ........................................................ 9

2.3 Band gap control in active elastic metamaterial plate ..................................................... 20

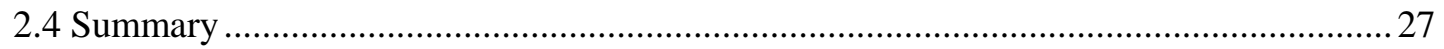

Chapter 3 Experimental Demonstration of the Tunable Wave Band Gaps ........... 28

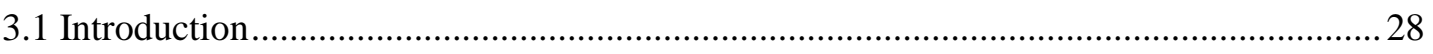

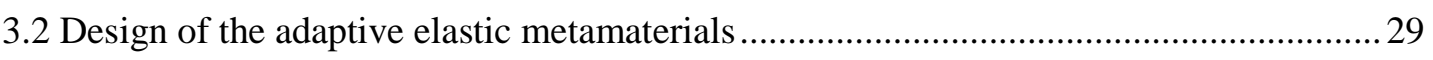

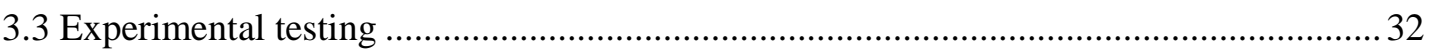

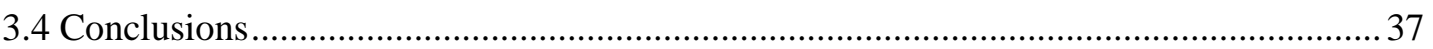

Chapter 4 An Adaptive Metamaterial Beam for Extremely Broadband Wave

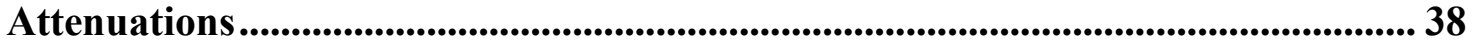

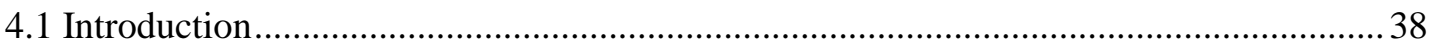

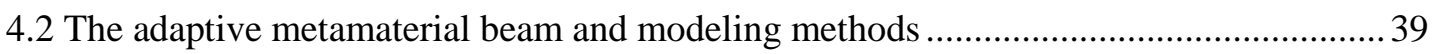

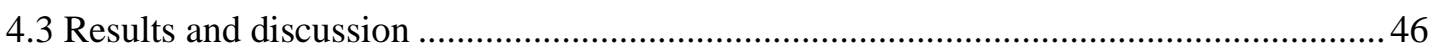

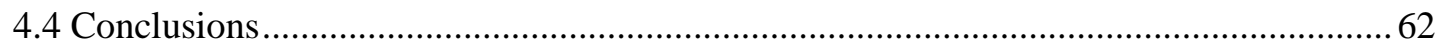

Chapter 5 A Hybrid Elastic Metamaterial with Negative Mass Density and

Tunable Bending Stiffness .......................................................................................................... 65

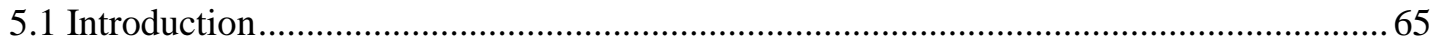

5.2 Design and multi-physical modeling of the adaptive hybrid metamaterial beam ............. 66

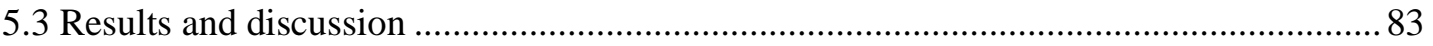

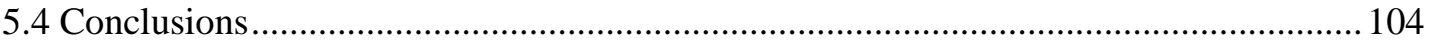




\section{Chapter 6 An Active Elastic Metamaterials for the Trajectory Control of Flexural}

Waves.

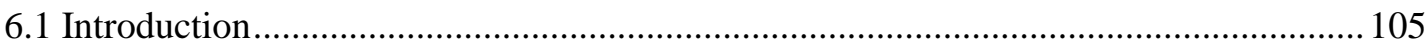

6.2 A transformation method of controlling flexural waves in plates................................. 106

6.3 Mapping design of a beam steering of flexural waves............................................... 111

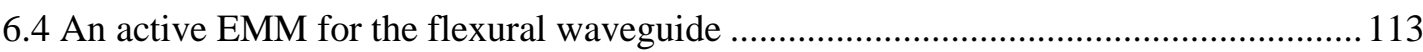

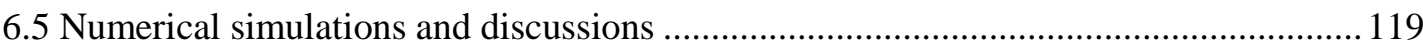

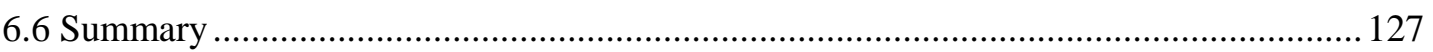

\section{Chapter 7 A Programmable Metasurface for Real Time Wavefront Control of}

Broadband Elastic Waves ....................................................................... 128

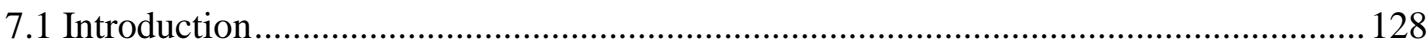

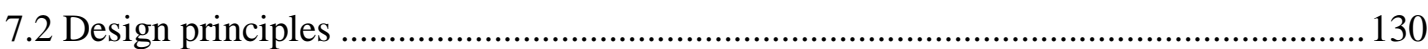

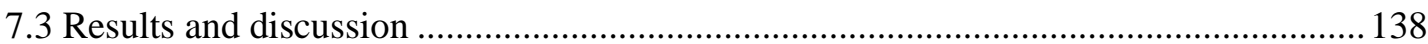

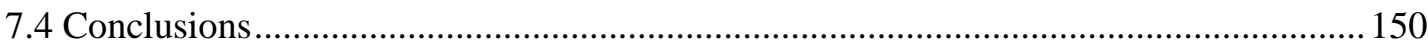

Chapter 8 Conclusions ......................................................................................... 152

Reference..................................................................................................................... 154

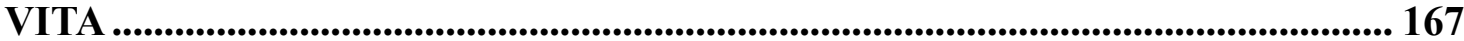




\section{Abstract}

Over the past two decades, an extensive research effort has been devoted to elastic metamaterials, structured artificial materials at subwavelength scales, for elastic wave manipulations in solids. Due to the extreme values of material parameters, negative and/or positive, they achieved, the applications can range from wave and/or vibration attenuations, wave guiding and imaging, enhanced sensing, to invisible cloaking. However, conventional passive metamaterials have limitations, i.e. they can only be operated in narrow frequency regions and their functions are usually locked into space or with minor tunabilities once fabricated, lacking real time reconfigurabilities. Those limitations strongly hinder them from practical usages. With the rapid development of smart materials and structures, more and more intelligent elements are being introduced into wave propagation, vibration and sound control systems. The piezoelectric shunting technique is one well known method that receives considerable attention. In this dissertation, by leveraging the circuit control concept, both analog and digital, we propose some circuit controlled active/adaptive/hybrid/programmable metamaterials and metasurfaces for unprecedent elastic wave manipulations. Analytical, numerically and experimentally approaches are combined throughout the dissertation to illustrate design concepts, characterize wave propagation properties, and valid the designs. Specifically, active elastic metamaterials with tunable stiffness in local resonators are first designed for tunable wave and/or vibration mitigations. We then extend this concept to achieve super broadband wave attenuations with frequency-dependent stiffness elements. By introducing the variable stiffness elements to the host medium, a hybrid metamaterial is developed for switched ON/OFF wave propagations and broadband negative refractions. Based on a developed 
approximate transformation method, an active metamaterial is designed and placed on a plate to achieve spatially varying effective mass densities for broadband elastic trajectory control. Finally, a programmable metasurface with ultrathin-thickness is demonstrated for broadband, real-time and multifunctional wavefront manipulations in a plate. The active, adaptive, hybrid, and programmable elastic metamaterials and/or metasurfaces are still in their infant stages. The examples presented in the dissertation are transformable to different length and time scales and could serve as efficient and powerful tools in exploring some unconventional wave phenomenon in solid structures, i.e. by using concepts in quantum mechanics, where passive approaches are significantly limited. The designs could also immediately open new possibilities in elastic wave control devices including, but not limited to structural health monitoring, stealth technology, active noise control, as well as medical instrumentation and imaging. 


\section{Chapter 1 Introduction}

\subsection{Acoustic/elastic metamaterials}

Metamaterials are artificial structures, typically periodic, composed of small metastructures that, in the bulk, behave like a continuous material with unconventional effective properties. The science of wave propagating in periodic structures goes back decades [1]; however, our modern appreciation of the use of engineered structures to control wave properties began with photonic and phononic crystals [2-7]. Research in this area rapidly expanded with the understanding that relatively simple, but subwavelength, building blocks can be assembled into structures that are similar to continuous materials, yet have unusual wave properties that differ substantially from those of conventional media.

In acoustics, the first artificial metamaterial used rubber-coated lead spheres distributed into epoxy matrix to create locally resonant and deeply subwavelength structures that responded to incident acoustic waves (Fig. 1-1 (a)) [8]. An assembly of these metastructures into a bulk metamaterial exhibited peculiar, but useful, acoustic properties such as negative mass density [9-22]. Building on this work, the field of acoustic metamaterials has focused on developing artificial meta-structures that are capable of controlling the propagation of low-frequency sound in new ways, made possible by the creation of another unusual material property such as negative modulus [23,24]. The emergence of acoustic metamaterials not only resolved the sample size problem but also introduced new functionalities not found previously. For instance, it is now possible to design membranetype acoustic metamaterials that can exhibit a favorable sound absorption/attenuation capability within $50-1000 \mathrm{~Hz}$ to break the mass density law of sound attenuation (Fig. 1-1 
(b)) [25-27]. Over the past decades, the field of acoustic metamaterials has branched out in many directions, and it has been shown that acoustic waves can be manipulated and controlled in ways not previously imagined.

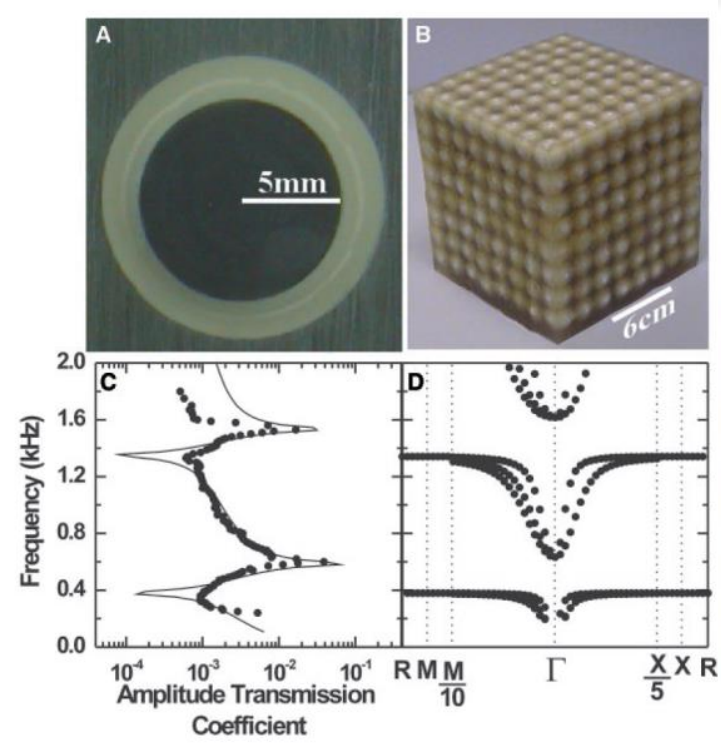

(a)

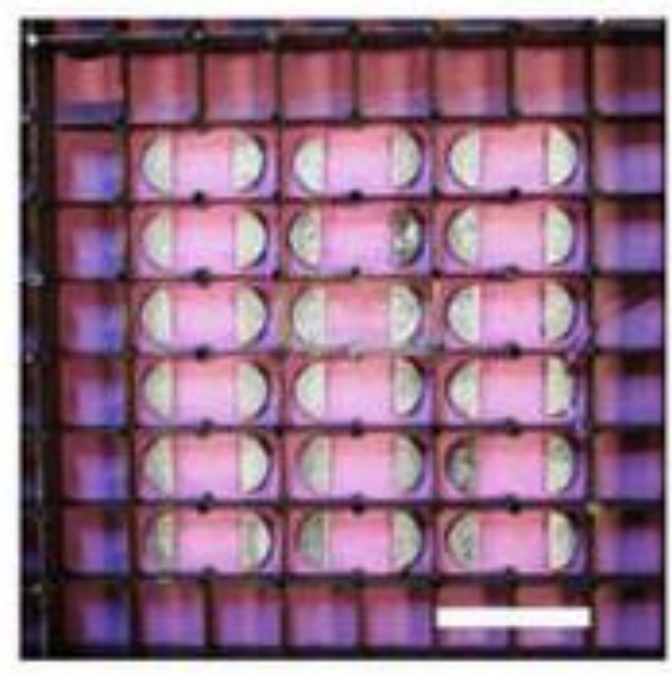

(b)

Figure 1-1 (a) Locally resonant acoustic metamaterial with rubber-coated lead spheres distributed into epoxy matrix [8]; (b) Membrane-type acoustic metamaterial [25]

Like their acoustic counterpart, elastic metamaterials (EMM), refers to a class of periodic structural (mechanical) materials, was also investigated for controlling the propagation of elastic/mechanical waves (longitudinal and transverse waves) (Fig. 1-2) [28-41]. Due to their subwavelength properties, elastic metamaterials are now viewed as promising candidates for many novel dynamic applications including low-frequency vibration shielding, guided wave attenuation, earthquake mitigation, mechanical wave guides, elastic wave absorption and harvesting, negative refraction, and elastic wave cloaking [28-41]. For instance, in the two-dimensional plate-like structure, the wave and 
vibration mitigation in low-frequency range has been realized by embedding soft inclusion, surface-bonding pillars, inserting local resonators within a lattice, among other configurations [18]. This is an open area of research that promises to continue to attract the attention and imagination not only of scientists, but also of the general engineering as a whole.
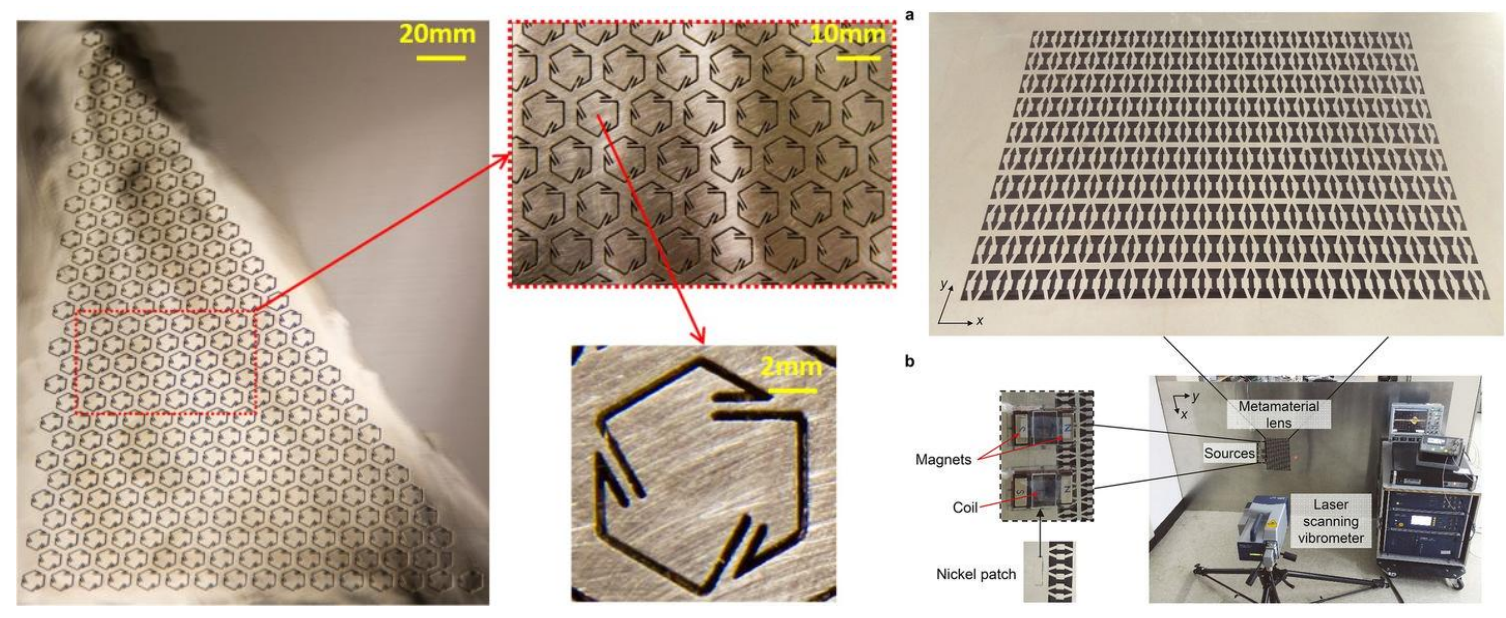

Figure 1-2 Elastic metamaterials for negative refraction and subwavelength imaging applications $[29,41]$

However, the major limitation of the current acoustic/elastic metamaterials with the locally resonant mechanisms is that the operation frequency range is fixed and limited to a narrow frequency band. As a result, their wave attenuation ability is significantly reduced if the wave frequency is not near the resonant frequency. Actively controlling the position and size of the bandgap frequency range in real-time is very difficult, if not impossible, for passive acoustic/elastic metamaterials [42-45]. One of the most pronounced challenges in developing acoustic/elastic metamaterial as practical devices is the ability to tune their wave performance without requiring physical microstructural modifications [45-48]. 


\subsection{Tunable phononic crystals and metamaterials}

For all these aforementioned reasons, there has been a growing effort to explore adaptive or active acoustic/elastic metamaterials, which could potentially overcome the challenges described above and increase their effectiveness in relevant applications. The term 'adaptive' or 'active' is used quite generally to indicate the meta-structures that can provide energy to the impinging wave and feedback to the elastic/acoustic system by integrating adaptive or smart materials into the unit cell, and therefore the effective properties of the adaptive metamaterial can be tuned or adapted online for complex system.

Among currently available adaptive materials, shunted piezoelectric materials provide an ideal platform to tune and control elastic/acoustic properties of a metamaterial in a compact way. This is because of their strong response to electrical signals which can be controlled with relatively simple electronics [49-73]. The piezoelectric effect allows energy to be exchanged between the mechanical and electrical domains so that, when a piezoelectric element is shunted through an electrical circuit, it behaves as a variable stiffness element that can be used to convert mechanical energy. In a more general representation, this coupled electro-mechanical mechanism can be employed to alter the dynamic acoustic and mechanical behavior of the structure. The piezoelectric shunting technique was first introduced by Forward (1979) to damp mechanical vibrations in optical systems [49], while Hagood and von Flotow (1991) provided the first analytical formulation for the passive electrical shunts [50]. Since their pioneering works, an array of electrical shunting designs have been proposed to improve the acoustic and dynamic mitigation performance of structures. In these works, piezoelectric components connected to inductor-resistor (LR) shunt circuits appear as a promising passive technique for noise 
and vibration control in dynamic structures [52-61]. This type of shunt generates an electrical resonance in the piezoelectric patch. If this electrical resonance is tuned to one of the structural modal frequencies, a considerable damping effect of the corresponding mode can be achieved. Generally, the resonant shunts with simple inductance-resistance components suffer from a drawback because their mitigation performance is limited to a relatively narrow frequency range and therefore very sensitive to parameter variations in the system. To overcome this problem, many efforts on piezoelectric metastructures with resonant shunts have been made to enlarge the wave attenuation region by introducing electric transmission line [74], implementing amplifier-resonator feedback circuits [75] and networks of different resonant shunts [76] (Fig. 1-3).
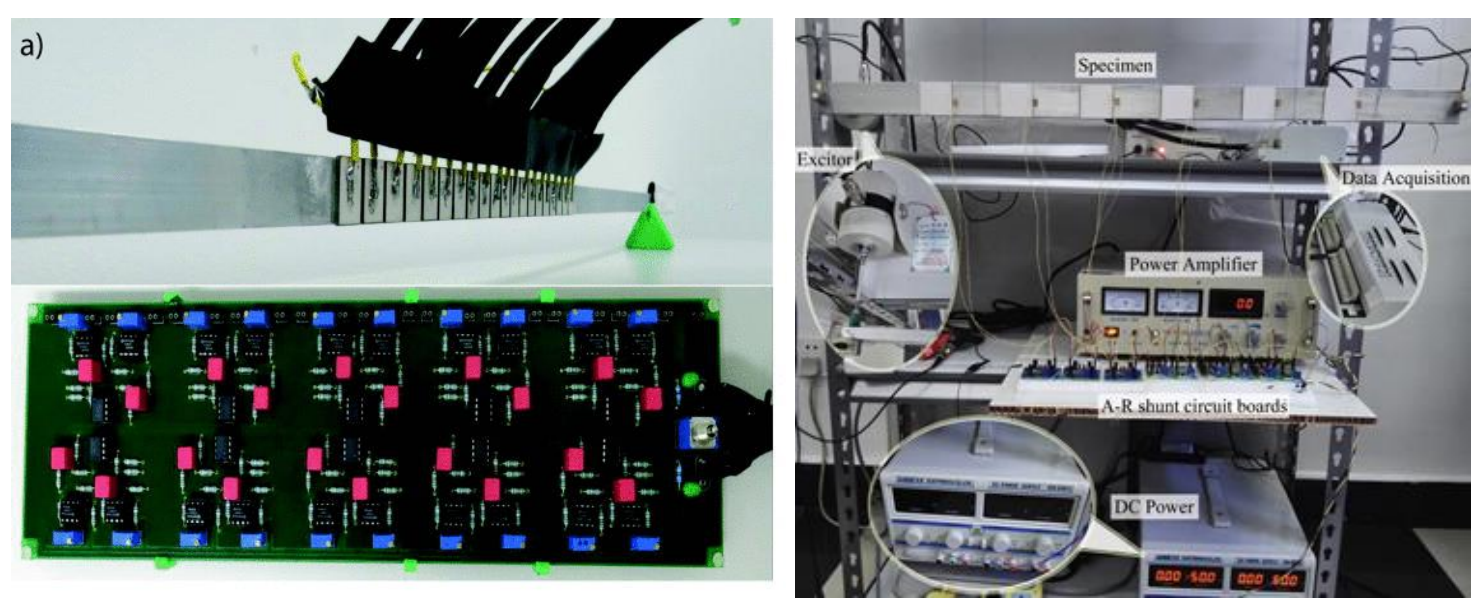

Figure 1-3 Piezoelectric phononic crystals with resonant-type shunting circuits $[74,75]$

As another type of shunting strategy to enlarge the wave attenuation region, negative capacitance shunts have been proposed and investigated [62-67]. Theoretically, the negative capacitance of the shunting circuit eliminates the piezoelectric patch's inherent capacitance such that the remaining resistance can optimally convert acoustic and/or elastic wave energy in a broadband frequency region. The applications of periodic piezoelectric 
patches with negative capacitance shunts have also been proposed for various spatial wave control, which include the flexural wave focusing [77], adaptive wave filtering with tunable Kirigami lattices [78], and wave directionality manipulations [79] (Fig. 1-4).

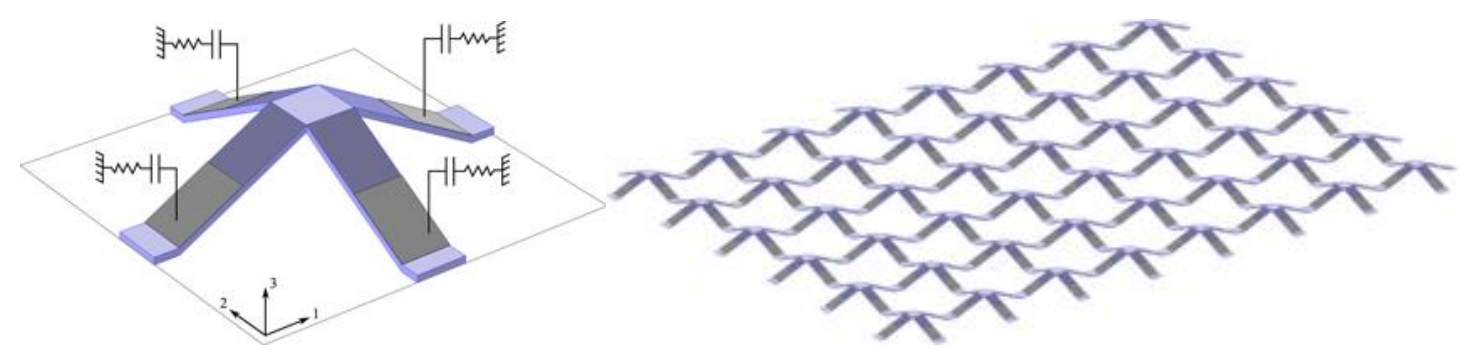

Figure 1-4 Piezoelectric metamaterials with negative capacitance shunting circuits [78]

\subsection{Motivations and outline}

By embracing the current state of art in smart materials and structures into elastic metamaterial building blocks, the dissertation will explore new opportunities provided by active elastic metamaterials for broadband, reconfigurable and multifunctional wave control applications. Analytical, numerical and experimental approaches are highly integrated in the dissertation to demonstrate those wave manipulation functionalities not only in time and frequency spectrum but also in a spatial domain.

This dissertation is organized as follows. In Chapter 2, a new class of active elastic metamaterials with negative capacitance piezoelectric shunting is presented. It can be found that the location and the extent of induced band gap of the elastic metamtaerial can be effectively tuned by using shunted piezoelectric patch with different values of negative capacitances, especially for extremely low-frequency cases. In Chapter 3, we experimentally demonstrate an active metamaterial tube controlled by electrical circuits. The tunable bandgap capacity, as high as $45 \%$, is physically realized by using both hardening and softening shunted circuits. To illustrate a practical application, transient 
wave propagation tests of the adaptive metamaterial subjected to impact loads are conducted to validate their tunable wave mitigation abilities in real-time. In Chapter 4, we report a new class of adaptive metamaterial beams with hybrid shunting circuits to realize super broadband Lamb wave band gaps at an extreme subwavelength scale. It is shown theoretically and numerically that by properly modifying the shunting impedance, the adaptive mechanical mechanism within the tunable resonator can produce high-pass and low-pass wave filtering capabilities for the zeroth-order anti-symmetric Lamb wave modes. In Chapter 5, we propose an adaptive hybrid metamaterial that possesses both a negative mass density as well as an extremely tunable stiffness by properly utilizing both the mechanical and electric elements. The programmed flexural wave manipulations, broadband negative refraction and waveguiding are then illustrated through threedimensional (3D) multi-physical numerical simulations in hybrid metamaterial plates. Our numerical results demonstrate that the flexural wave propagation can essentially be switched between "ON/OFF" states by connecting different shunting circuits. In Chapter 6, we propose a transformation method to derive the material properties of a flexural waveguide, and implement its functionality based on a design of active elastic metamaterials. The numerically demonstrated flexural waveguide can not only steer an elastic wave beam as predicted from the transformation method, but also exhibit various unique properties including extraordinary wave beam deflection and tunabilities over a broad frequency range and various steering directions. In Chapter 7, we introduce a programmable elastic metasurface for the first time with self-sensing-and-actuating units, allowing to adapt and reprogram its wave control functionalities in real time. The proposed metasurface is concretized numerically and experimentally by achieving real time ray 
steering of flexural waves in a host plate. Various other significant demonstrations have been included to strongly illustrate the multifunctional adaptability of the design. In particular, one-way non-reciprocal blocking of waves is observed experimentally whereas skin cloaking of voids is tested numerically. Operability across broad wave frequency ranges is demonstrated. 


\section{Chapter 2 An Active Elastic Metamaterial with Tunable Wave}

\section{Band Gaps}

\subsection{Introduction}

In this chapter, the concept of the active elastic metamaterial is first proposed to achieve band gap control through the use of negative capacitance shunted piezoelectric patches. An active mass-in-mass lattice system with the inner spring connected to the negative capacitance piezoelectric shunting is studied to demonstrate the flexible band gap control mechanism over a wide frequency range. This promising application is then demonstrated with an active elastic metamtaerial plate integrated with negative capacitance shunted piezoelectric patches for longitudinal and bending waves. The result reveals that using negative capacitive shunting to tune the band gap is very effective, although improvements can be further made at this point.

\subsection{Band gap control in active elastic metamaterials}

\subsubsection{Negative capacitance piezoelectric shunting}

Figure 2-1 shows the circuit that influences/controls the elastic parameters of the piezoelectric sample, designated as gyrator circuit A. It consists of a capacitor, resistors and an operational amplifier. A piezoelectric sample disconnected from the circuit is shown in Fig. 2-1(a). A static stress is applied to this sample, which induces voltage $V_{p}$, and the capacitance of the piezoelectric element is denoted as $C_{p}$ for open circuit. The magnitude of strain polarization is shown by the length of the arrow. The polarization induces negative charge on the upper electrode, and positive charge on the lower electrode. When the piezoelectric sample is connected to the circuit, as shown in Fig. 2-1(b), the non-inverting 
and inverting of the operational amplifier $(\mathrm{OA})$ are $V_{i n}^{+}$and $V_{i n}^{-}$, respectively. Through straightforward circuit analysis, the equivalent capacitance of this circuit is determined as $-C_{n}=-\frac{R_{1}}{R_{2}} C$

which $C_{n}$ is always a positive value.

Thus circuit can be considered as a negative capacitance circuit with a capacitance $C$ to increase the elastic properties. Stable operation of the circuit negative feedback requires the condition $|C|<\left|C_{p}\right|$ for the circuit A. Another type of negative capacitance circuit denoted as circuit $\mathrm{B}$, which decreases the elastic properties, is that the sample electrode is connected to the inverting input of the OA, and Eq. (2.1) holds for this circuit also, and the stability condition requires $|C|>\left|C_{p}\right|$. Equation (2.1) shows that any negative capacitance can be obtained by appropriately selecting the circuit parameters. Moreover, different tunings can be performed by simply varying one resistor of the synthetic circuit.

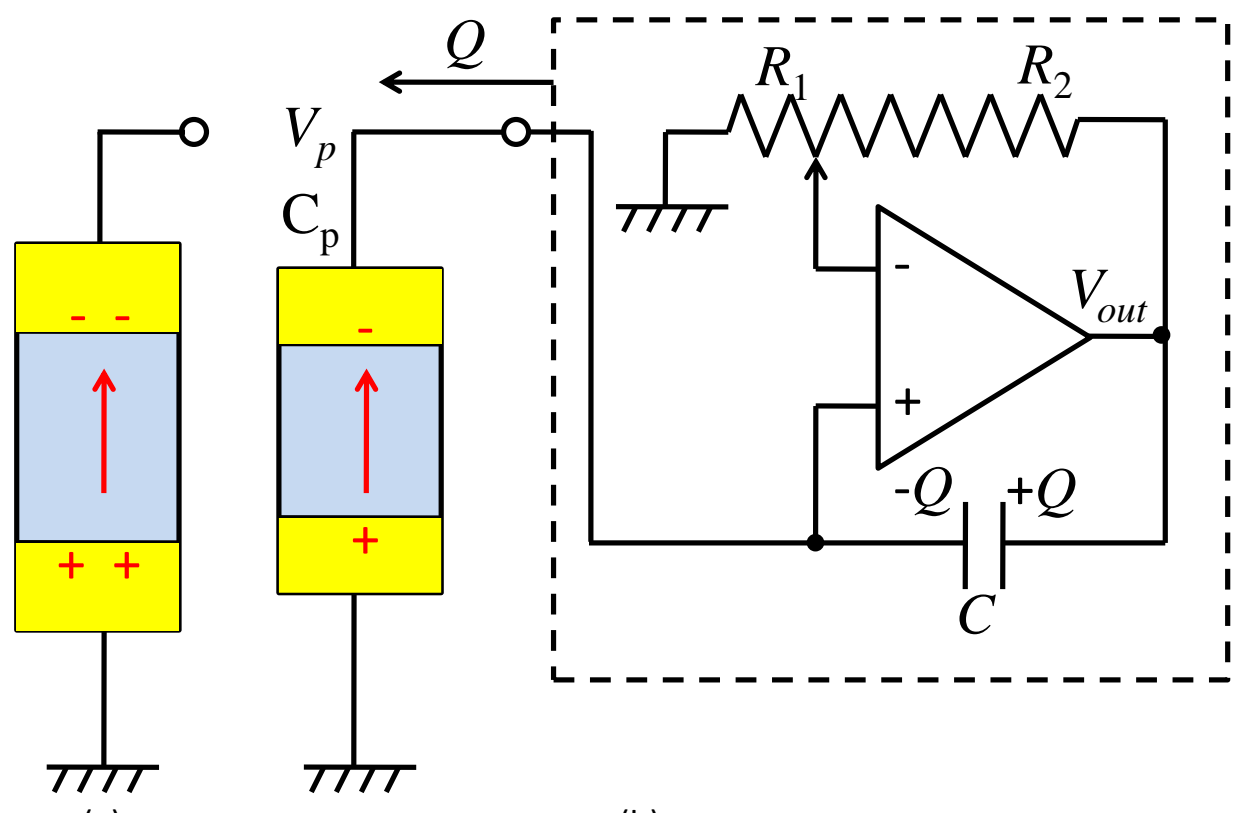

(a)

(b) 
Figure 2-1 Principle of operation of the negative capacitance circuit A, (a) piezoelectric sample disconnect from the circuit; (b) piezoelectric sample connected with the circuit

Now consider a thin, shunted piezoelectric patch is assumed to be shunted and connected to a parallel negative capacitance $-C_{n}$, which is schematically illustrated in Fig. 2-2 (a). It is readily found that the shunted system is equivalent to a 'simple' transducer with a lower capacitance, as shown in Fig. 2-2(b).

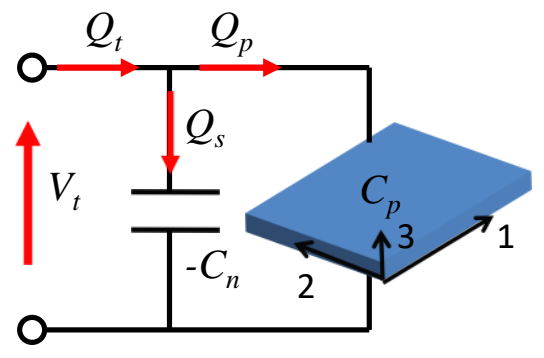

(a) Piezo patch

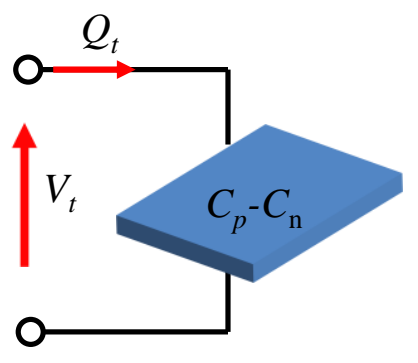

(b) Equivalent piezo patch

Figure 2-2 (a) piezoelectric patch connected to a parallelnegative capacitance $-C_{n}$; (b) equivalent piezoelectric patch

Indeed, the total electrical charge $Q_{t}$ flowing in the system is given by

$Q_{t}=Q_{p}-C_{n} V$

and, from Eq. (2.2), the equivalent system is governed by the constitutive relation in Laplace domain when the piezoelectric element is loaded uniaxially along the 1 axis as

$$
\left[\begin{array}{l}
I \\
\sum_{11}
\end{array}\right]=\left[\begin{array}{cc}
s\left(C_{p}-C_{n}\right) & s d_{31} A \\
d_{13} / h & s_{11}
\end{array}\right]\left[\begin{array}{l}
V \\
E_{11}
\end{array}\right]
$$

where $s=i \omega$ is the Laplace operator, $C_{p}=\frac{\varepsilon_{33} A}{h}, \varepsilon_{33}$ is the dielectric constant of the material, $d_{31}$ is the piezoelectric constants, $s_{11}$ is the compliance matrix of the 
piezoelectric patch, $\sum_{11}$ and $E_{11}$ are stress and strain along 1 axis direction, $A$ and $h$ are the electrode area and thickness of the piezoelectric patch, respectively, the 1 axis is the direction of the length of the PZT patch, the 3 axis is the direction of the thickness of the patch, and the 2 axis is perpendicular to both the 1 and 3 axes, as shown in Fig. 2-2(a). According to Hagood and von Flotow (1991), modified effective Young's modulus of the shunted patch can be expressed according to the following expression, which is independent of frequency $\omega$, as

$$
E_{p}^{S U}(\omega)=E_{p}^{E} \frac{C_{p}^{T}-C_{n}}{C_{p}^{T}\left(1-k_{31}^{2}\right)-C_{n}}
$$

where $k_{31}=d_{31} / \sqrt{s_{11} \varepsilon_{33}}$ denotes the electro-mechanical coupling coefficient, $E_{p}^{E}$ is Young's modulus of the piezoelectric material when the shunting network is in a short circuit configuration, $C_{p}^{T}=C_{p}$ is the electrical capacitance of the piezoelectric material at constant stress. The relationship between the open circuit Young's modulus and short circuit Young's modulus is

$$
E_{p}^{E}=E_{p}^{D}\left(1-k_{31}^{2}\right)
$$

A significant result from Eq. (2.4) is that the shunt has the ability to modify elastic properties, and thus may be used to control the dynamic behavior of the piezoelectric material.

Figure 2-3 shows the normalized effective modulus $E_{\text {eff }}^{*}=E_{p}^{S U} / E_{p}^{D}$ in function of the negative capacitance ratio (NCR), which is defined as $\lambda=-\frac{C_{n}}{C_{p}}$. The decrease of the effective modulus is corresponding to the negative capacitance circuit A, as shown in Fig. 2-1 (a), and the increase of the effective modulus is related to the negative capacitance 
circuit B. It can be found that for all frequencies the effective modulus of the shunted piezoelectric patch can vary between the open circuit and short circuit conditions, depending on the magnitude of capacitance. The normalized effective stiffness modulus is equal to the short circuit stiffness for large values of $\lambda$ and equal to the open circuit stiffness for small values of $\lambda$. For $\lambda$ equals to -1.0 , the effective modulus becomes zero. For $\lambda$ near unity, the normalized effective modulus exhibits an asymptotic behavior. When $\lambda$ approaches to $-\left(1-k_{31}^{2}\right)$ from small values, the effective modulus approaches positive infinity. However, when $\lambda$ approaches to $-\left(1-k_{31}^{2}\right)$ from large values, the effective modulus become to be negative value, and then approaches negative infinity. The tunable modulus behavior of the shunted piezoelectric element will be used for the concept design of the active acoustic material.

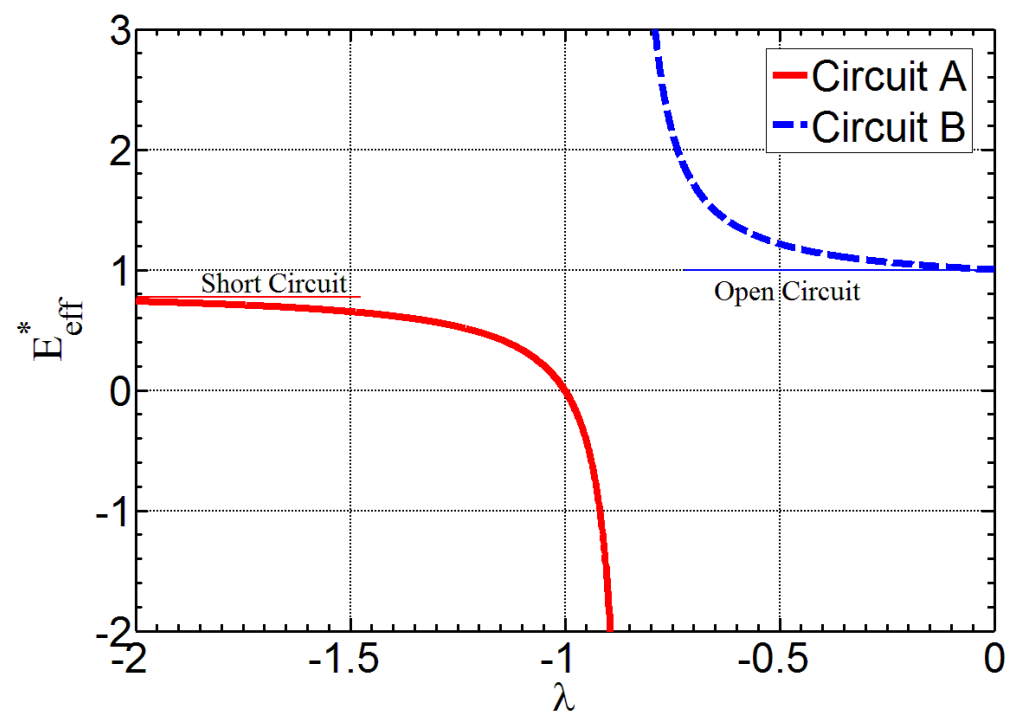

Figure 2-3 Normalized effective modulus of piezoelectric patch with different NCRs in circuits A and B, respectively. 


\subsubsection{Active mass-in-mass system}

The one-resonator mass-in-mass model has been proposed by Huang et al. (2009) to interpret the effective mass density of the one-dimensional elastic metamaterial. In this study, we will consider the same one-dimensional lattice consisting of active mass-in-mass units as shown in Fig. 2-4. The unit-cells are placed periodically at a spacing of $L$. The two rigid masses are denoted as $m_{1}$ and $m_{2}$, respectively. The two spring constants $k_{1}$ and $k_{2}$ represent the interactions among the masses. Note that, in each unit cell, the inner spring constant with the negative capacitance piezoelectric shunting is denoted as $k_{2}(\lambda)=\frac{E_{p}^{S U} A_{p}}{l_{p}}$, which is a function of NCR $\lambda$, where $A_{p}$ and $l_{p}$ are the cross sectional area and length of the piezoelectric patch, respectively.
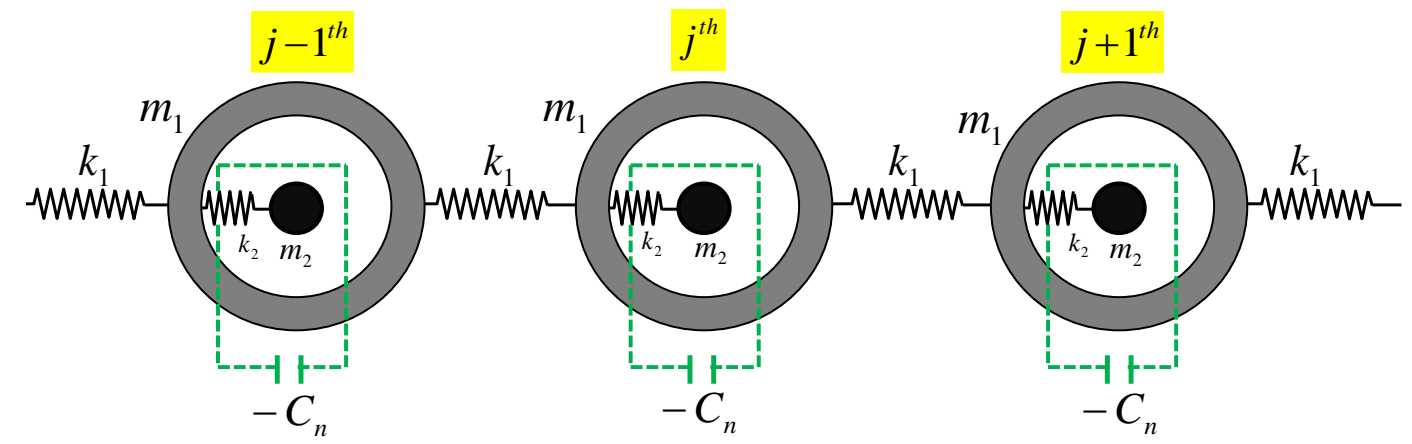

Figure 2-4 Active mass-in-mass lattice system with inner spring to represent negative capacitance piezoelectric shunting

For harmonic wave propagation in the mass-in-mass lattice system, the equations of motion for the $j$-th unit cell can be expressed as

$$
m_{1} \frac{d^{2} u_{1}^{(j)}}{d t^{2}}+k_{1}\left(2 u_{1}^{(j)}-u_{1}^{(j-1)}-u_{1}^{(j+1)}\right)+k_{2}(\lambda)\left(u_{1}^{(j)}-u_{2}^{(j)}\right)=0
$$


$m_{2} \frac{d^{2} u_{2}^{(j)}}{d t^{2}}+k_{2}(\lambda)\left(u_{2}^{(j)}-u_{1}^{(j)}\right)=0$

where $u_{\alpha}{ }^{(j)}$ represents the displacement of mass " $\alpha$ " in the $j$-th cell. The harmonic waveform of the displacement at the $j$-th cell is

$u_{\alpha}^{(j)}=B_{\alpha} e^{i(q x-\omega t)}$

where $B_{\alpha}$ is complex wave amplitude, $q$ is wave number, and $\alpha=1$ and 2 denoting the two masses. Substitution of Eq. (2.8) into Eqs. (2.6) and (2.7) yields two homogeneous equations from which the dispersion equation can be obtained as

$$
m_{1} m_{2} \omega^{4}-\left[\left(m_{1}+m_{2}\right) k_{2}(\lambda)+2 m_{2} k_{1}(1-\cos (q L))\right] \omega^{2}+2 k_{1} k_{2}(\lambda)(1-\cos (q L))=0
$$

It is evident that the dispersion curves (the plots of $\omega$ vs $q L$ ), of the resonator system are influenced by a number of parameters including the effective modulus $k_{2}(\lambda)$.

In this study, wave band gaps in the active mass-in-mass system are investigated for different active spring constant values. Attention is focused on the roles played by the active spring parameter $k_{2}(\lambda)$ which is function of the NCR $\lambda=-\frac{C_{n}}{C_{p}}$.
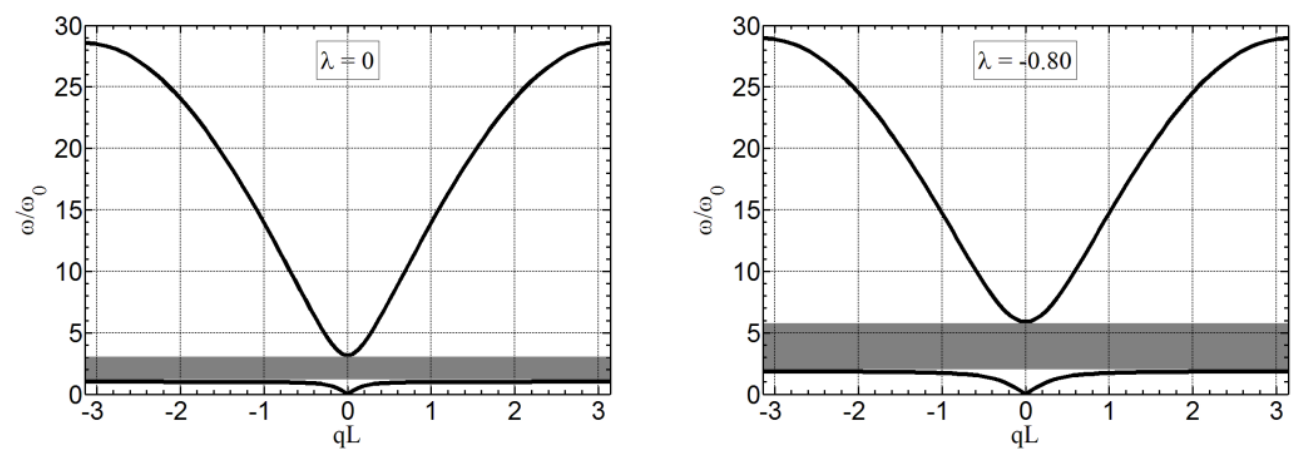

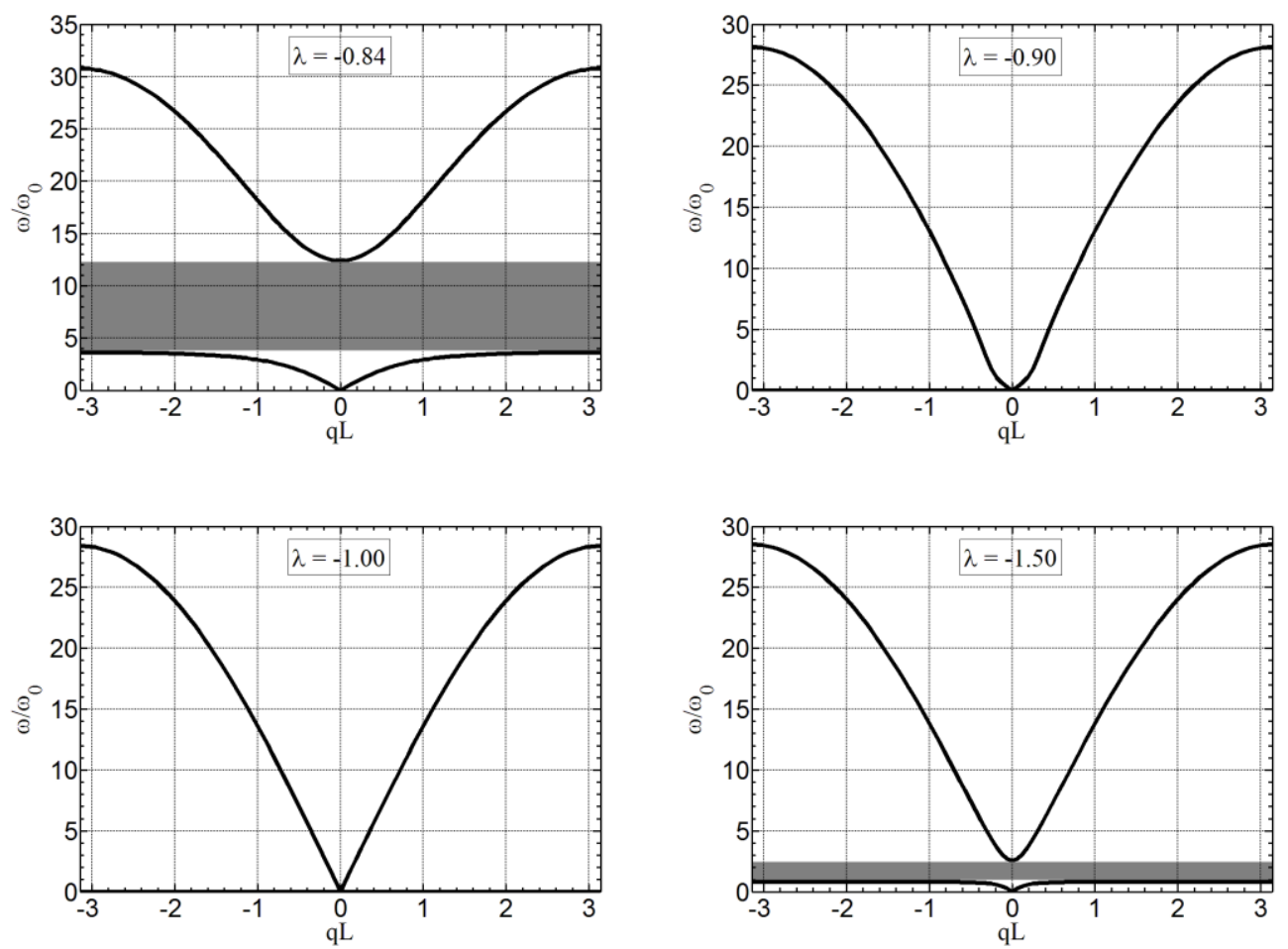

Figure 2-5 Band gap of the active mass-in-mass lattice system with different NCRs

The variation of band gap corresponding to different NCRs is shown in the shaded regions in Fig. 2-5. In the example, $m_{2} / m_{1}=9, k_{1}=1000 \mathrm{~N} / \mathrm{m}$, and the inner effective spring $k_{2}$ is calculated from the longitudinal deformation in the shunted piezoelectric $(\mathrm{PZT}-5 \mathrm{H})$ beam. The material properties of the piezoelectric patch are $\varepsilon_{33}=3.10 E-8 F / m, d_{31}=-2.74 \mathrm{E}-10 \mathrm{C} / \mathrm{m}^{2}, E_{P}=60.6 \mathrm{GPa}, A_{p} / l_{p}=6 E-8 \mathrm{~m}^{-1}$. In the case, the effective modulus of piezoelectric material with open circuit is represented by $k_{2}(\lambda=0)=44.6 \mathrm{~N} / \mathrm{m}$ with $k_{2}(0) / k_{1}=0.0446$. In the figures, $\omega_{0}=\sqrt{k_{2}(0) / m_{2}}$ is the local resonance frequency of the resonator system with the open circuit. Figure 5(a) shows the band gaps when $\lambda=0$, i.e., the shunting circuits are open. The band gap is in the range of $\omega=\omega_{0} \sim 3.2 \omega_{0}$. The effective modulus of shunted PZT patch will change with the 
change of the value of connected negative capacitances, which induces band gap changes in the elastic metamaterial. Figure 2-5(b) shows the band gap of the elastic metamaterial when $\lambda=-0.8$ and the spring stiffness ratio $k_{2}(-0.8) / k_{1}$ increases. Comparing the two diagrams, it is found that the width of the band gap is slightly enlarged, and the band gap range is shifted from the original $\left(\omega_{0} \sim 3.2 \omega_{0}\right)$ to $\left(1.8 \omega_{0} \sim 5.9 \omega_{0}\right)$ as shown in Fig. $2-5(\mathrm{~b})$. Figure 2-5(c) shows the band gap of the elastic metamaterial when $\lambda=-0.84$ and the spring stiffness ratio become significantly large. The width of new band gap is significantly enlarged from the original $\left(\omega_{0} \sim 3.2 \omega_{0}\right)$ to $\left(3.6 \omega_{0} \sim 12.4 \omega_{0}\right)$. Therefore, the broadband frequency of the stop band could be tuned to be $\left(\omega_{0} \sim 12.4 \omega_{0}\right)$ by the active elastic metamaterial connecting to different negative capacitance such as $\lambda=0, \lambda=-0.8$ and $\lambda$ $=-0.84$. Figures $2-5(\mathrm{~d})$ and $2-5(\mathrm{e})$ show the band structure diagram of the active elastic metamaterial when $\lambda=-0.9$ and $\lambda=-1.0$, for which the spring stiffness ratio becomes negative and zero, respectively. In these two cases, the local resonance mechanism in the metamaterial is totally absent. The elastic metamaterial now functions as the conventional material and the low-frequency stop band disappears. When the NCR reduces further from $\lambda=-1.0$, the effective spring stiffness increases from zero to the value of the piezoelectric materials with short circuits. Figure 2-5(f) shows the band structure diagram of the elastic metamaterial when $\lambda=-1.5$, in which the spring stiffness ratio becomes positive again with $k_{2}(-1.5) / k_{1}=0.006$. It is expected that the lower frequency band gap can be achieved in the range of $\left(0.8 \omega_{0} \sim 2.6 \omega_{0}\right)$. If NCR reduces further from $\lambda=-1.5$, the band gap will not change significantly. 
To interpret the wave propagation and attenuation mechanism of the active elastic metamaterial with different NCRs, the displacement ratio of the two masses is also plotted in Fig. 2-6, which can be analytical determined as $\frac{U_{2}}{U_{1}}=\frac{k_{2}(\lambda)}{-m_{2} \omega^{2}+k_{2}(\lambda)}$. It can be found that the stop band is caused by out of phase motion between mass 1 and mass 2 . When $k_{2}(\lambda)$ becomes negative, the two masses will always move in the same phase, therefore, no stop band can be observed at this case. When $k_{2}$ becomes zero, the inner mass is motionless and wave propagation behavior will be the same as the wave propagation in single mass-spring $\left(m_{1}-k_{1}\right)$ system.

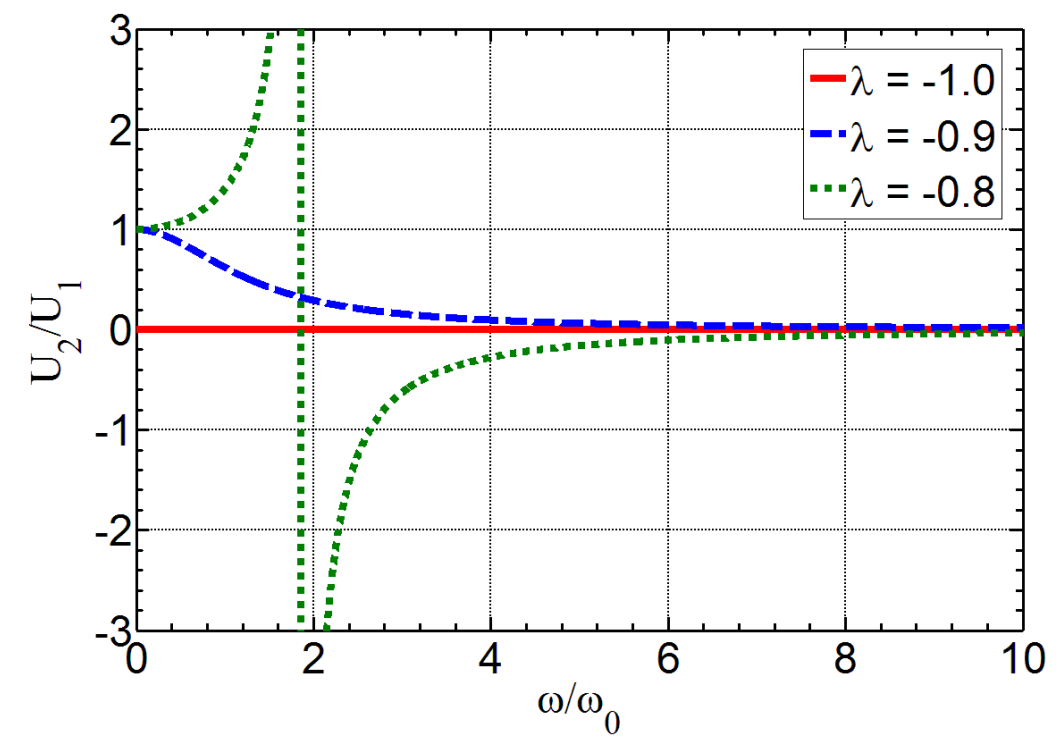

Figure 2-6 Displacement amplitude ratio $U_{2} / U_{1}$ of the inner mass $m_{2}$ to the outer mass

$$
m_{1}
$$

Finally, the general variations of band gaps of the elastic metamaterial are shown in the shaded region in Fig. 2-7. In the figure, the solid red line represents the upper bound of the 
stop band, and the dashed blue line is the lower bound of the stop band. When the shunted piezoelectric NRC $\lambda$ approaches -1.0 from negative infinity, the width of band gap of the elastic metamaterial will gradually decrease from the range $\left(\omega_{0} \sim 2.8 \omega_{0}\right)$ to zero. When $\lambda$ is tuned from -1.0 to $-\left(1-k_{31}^{2}\right)$, the elastic metamaterial will become a conventional material and the stop band disappears. Similarly, when the value of $\lambda$ is changed from $-\left(1-k_{31}^{2}\right)$ to 0 (open circuit), one can find that the location and bandwidth of band gaps are also distinctly changed. The bandwidth of stop band of the elastic metamaterial will greatly reduced from a large bandwidth to normal metamaterial band gap range $\left(\omega_{0} \sim\right.$ $\left.3.2 \omega_{0}\right)$. The result of the active elastic metamaterial provides a very promising solution to overcome the obstacle in the elastic metamaterial and actively tune the location and the width of band gaps.

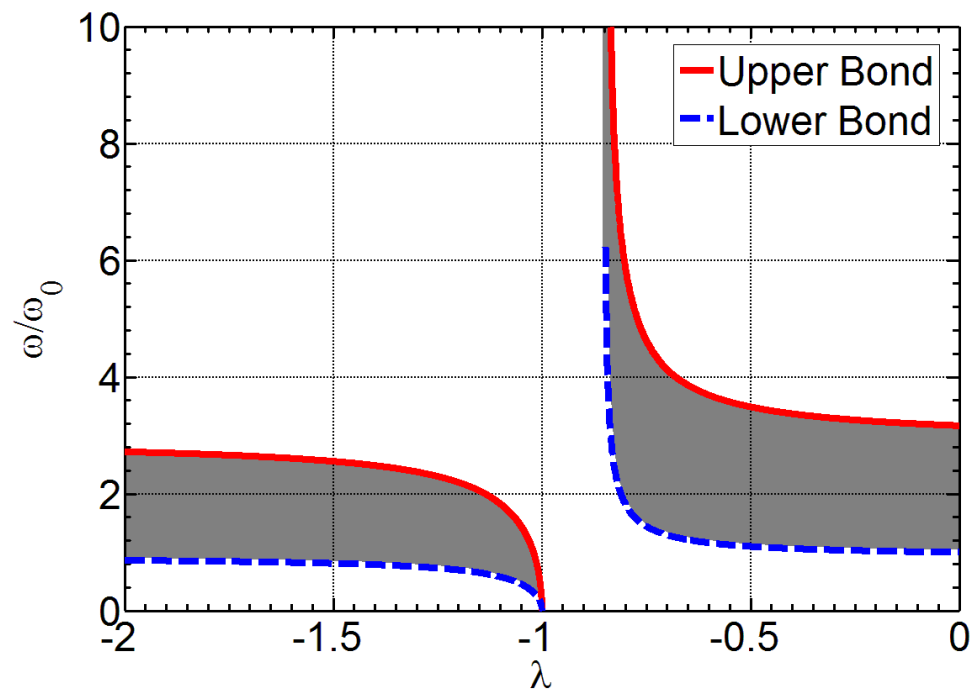

Figure 2-7 Band gap variation of the active mass-in-mass lattice system with different NCRs (bond should be bound) 


\subsection{Band gap control in active elastic metamaterial plate}

A thin metamaterial plate with periodic cantilever-mass microstructures was proposed for low-frequency bandgap applications for both in-plane and out-of-plane waves (Huang and Sun, 2009, Zhu et al, 2012). The low-frequency bandgaps in the elastic metamaterial plate are investigated numerically and experimentally for both in-plane extensional and out-of-plane flexural guided waves. In this study, we illustrate the practical application of how the periodic array of shunting piezoelectric patches bonded to the cantilever beam can function as the building block of an active elastic metamaterial. Effective modulus of such resonant unit can be numerically characterized by integrating the metamaterial plate with the shunting piezoelectric patch with different negative capacitance. Therefore, the local resonance frequencies of the metamaterial plate can be conveniently tuned through the proper selection of the electrical negative capacitance connected to each patch without modifying the microstructure.

Figure 2-8(a) shows the active elastic metamaterial plate with a periodic array of cantilever-masses bonded by shunted piezoelectric patches. The detailed microstructure in the unit cell is shown in Fig. 2-8(b). The plate behaves as a one-dimensional waveguide that supports the propagation of axial and transverse waves. In the low-frequency range, the behavior of the waveguide of the resonators can be conveniently described through the Euler-Bernoulli theory with piecewise elastic and mass properties. The negative capacitance shunts are applied to piezoelectric patches installed in a periodic array, which consists of a series of equally spaced piezoelectric patches. 


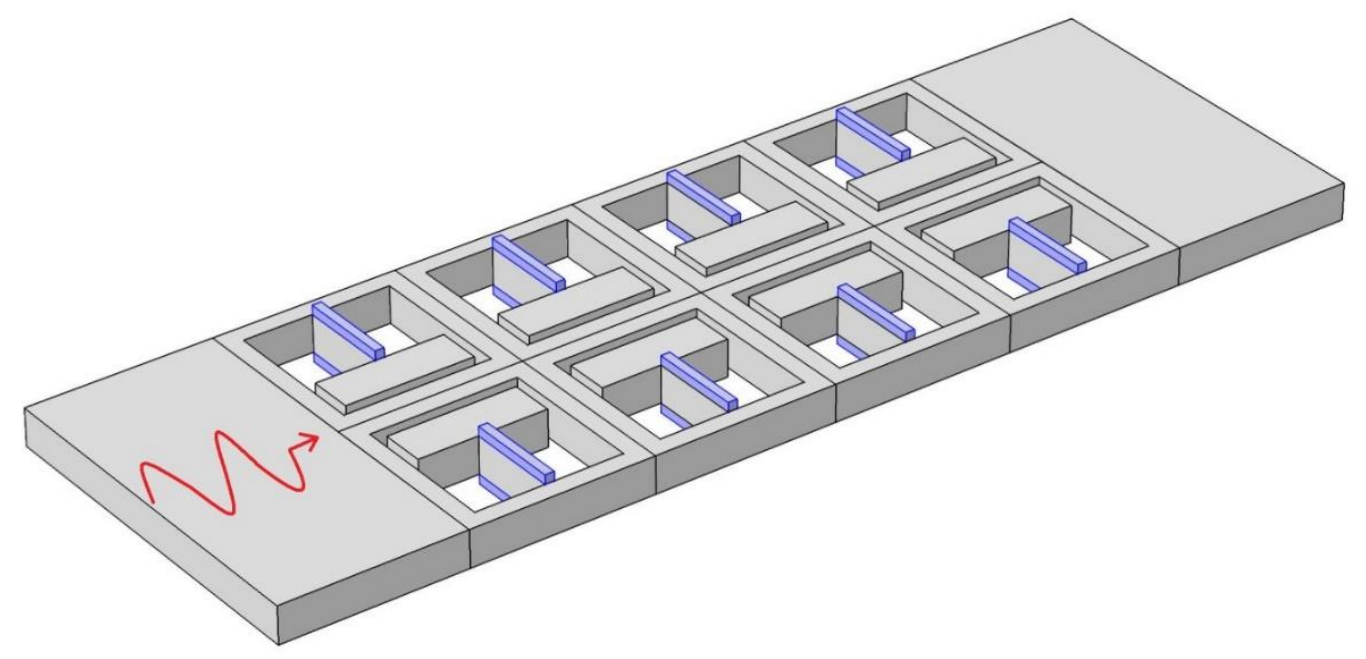

(a)

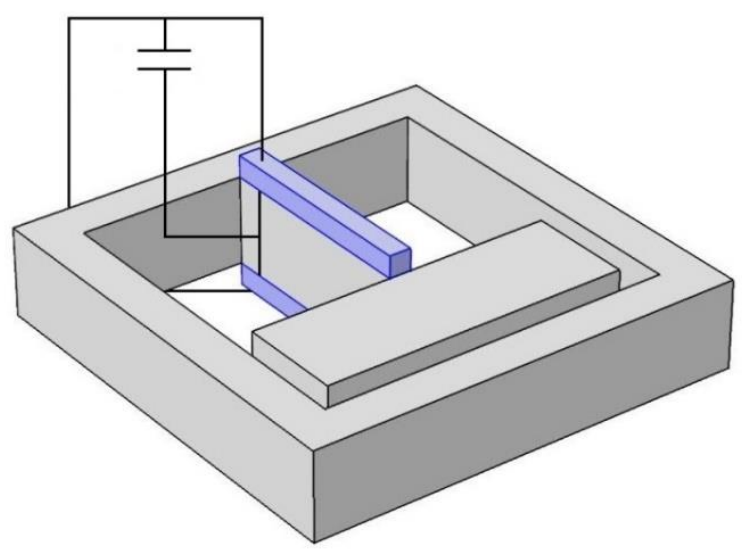

(b)

Figure 2-8 (a) Active elastic metamaterial plate with a periodic array of the cantilevermass system bonded by shunted piezoelectric patches; (b) detailed microstructure in the unit cell

The thin plate beam is made of stainless steel $\left(E_{s}=195 G P a, \rho_{s}=8000 \mathrm{~kg} / \mathrm{m}^{3}\right.$, $v_{s}=0.33$ ) with thickness $t_{s}=1 \mathrm{~mm}$ and width $b_{s}=0.45 \mathrm{~mm}$. The shunted piezoelectric materials is PZT-5H ( $\left.E_{p}=60.6 G P a, \rho_{p}=7500 \mathrm{~kg} / \mathrm{m}^{3}, v_{p}=0.34\right)$ with thickness $t_{p}=0.1 \mathrm{~mm}$, length $l_{p}=2.7 \mathrm{~mm}$ and width $b_{p}=0.45 \mathrm{~mm}$. The spacing of the adjacent unit cells 
is $a=5.6 \mathrm{~mm}$. Due to the geometric complexity of the microstructure, the finite element technique is employed with the aid of the commercial finite element software, ANSYS v13.0 to determine the band diagram of the active elastic metamateiral. In the numerical simulation, 3D solid element SOLID45 is chosen to model the thin steel plate and piezoelectric patches with effective modulus (based on the connected negative capacitance), and five elements are used in the thickness direction. Manual mesh strategy is used to better control mesh density and element shape for the complex interior cantilever-mass geometry.

The effective bending stiffness of the embedded composite cantilever beam can be determined as

$$
\begin{aligned}
& D_{b}=\frac{E_{p} b_{p} t_{p}^{3}}{6}+\frac{E_{p} b_{p} t_{p}\left(t_{p}+t_{c}\right)^{2}}{2}+\frac{E_{c} b_{c} t_{c}^{3}}{12} \quad \text { for bending wave } \\
& D_{l}=\frac{E_{p} b_{p} t_{p}^{3}}{6}+\frac{E_{c} b_{c} t_{c}^{3}}{12} \quad \text { for longitudinal wave }
\end{aligned}
$$

from which the effective Young's modulus of the composite beam can be determined and will be used in the finite element analysis. The dispersion relations of the active elastic metamaterial plates with surface-bonded the piezoelectric patches with different negative capacitance for in-plane extensional wave are plotted in Fig. 2-9. Attention is focused on the first band gap variation (identified in the shaded regions) with different NCRs $\lambda=-\frac{C_{n}}{C_{p}}$. The normalized wave number is defined as $q a$. Figure 2-9(a) shows the dispersion diagram and the first band gap when $\lambda=0$, i.e., the shunting circuits are open. In the figures, $\omega_{0}=\sqrt{k_{\text {eff }} / m_{2}}$ is the local resonance frequency of the cantilever resonator system with the open circuits for $k_{\text {eff }}=D_{l} / 3 l_{p}^{3}$. The band gap of the open shunting circuit 
is in the range of $\omega=\omega_{0} \sim 1.18 \omega_{0}$. The effective bending stiffness of the cantilever beam with the shunted PZT patch will change with the change of value of the connected negative capacitance, which induces the frequency range and bandwidth of the first band gap in the active elastic metamaterial plate. Figure 2-9(b) shows the dispersion diagram and the first band gap of the elastic metamaterial plate when $\lambda=-0.842$, in which the effective stiffness of the shunted piezoelectric patch increase as a positive value, therefore the effective bending stiffness of the cantilever beam will be increased. Comparing the band diagram with the open circuit, it is found that the band gap is shifted from the original band gap ( $\omega_{0}$ $\left.\sim 1.2 \omega_{0}\right)$ to $\left(1.5 \omega_{0} \sim 1.7 \omega_{0}\right)$ as shown in Fig. 2-9(b). However, the bandwidth of the stop band is not enlarged at this case. Figure 2-9(c) shows the band gap of the elastic metamaterial plate when $\lambda=-0.846$, in which the effective modulus of the composite beam becomes larger. The new band gap is observed to continuously shift to $\left(1.98 \omega_{0} \sim 2.2 \omega_{0}\right)$. Therefore, the stop band of the metamaterial plate could be tuned by changing the connected negative capacitance in a small value around the piezoelectric capacitance. Figure 2-9(d) show the band structure diagram and the first band gap of the elastic metamaterial plate when $\lambda=-0.863$, in which the effective stiffness of the shunted piezoelectric patch becomes a negative value, and therefore the effective bending stiffness of the cantilever beam will be decreased. Comparing the band diagram with the open circuit, it is found that the band gap is lowered from the original band gap $\left(\omega_{0} \sim 1.2 \omega_{0}\right)$ to $\left(0.61 \omega_{0} \sim 0.84 \omega_{0}\right)$. Figures 2-9(e) and 2-9(f) show the band structure diagram and the first band gap of the elastic metamaterial plate when $\lambda=-0.8585$ and $\lambda=-0.85$, respectively, in which the effective stiffness of the shunted piezoelectric patch is reduced further in a negative value. It can found that the extremely low-frequency band gaps can be observed 
at range of $\left(0.27 \omega_{0} \sim 0.73 \omega_{0}\right)$ and $\left(0.16 \omega_{0} \sim 0.7 \omega_{0}\right)$ for both cases. It is also interesting to notice that the width of the stop band becomes wider and is very sensitive to the change of the negative capacitance.
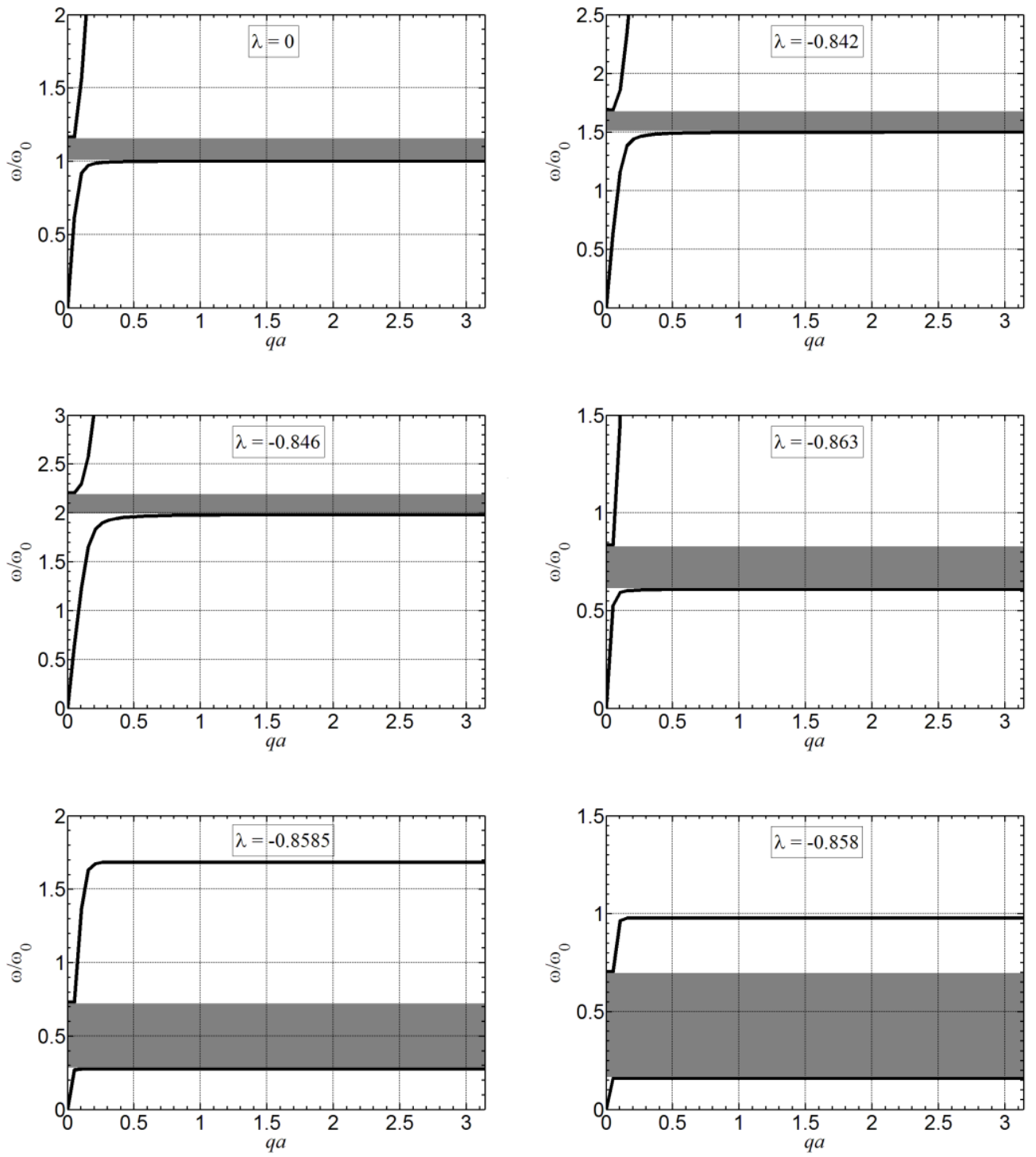

Figure 2-9 Band gap variation of the active elastic metamaterial plate with different

NCRs for in-plane longitudinal wave 
Figure 2-10 shows the band gap variation (identified in the shaded regions) of the active elastic metamaterial plate with surface-bonded piezoelectric patches with different NCRs $\lambda=-\frac{C_{n}}{C_{p}}$ for the out-of-plane bending wave. Figure 2-10(a) shows the band gaps when $\lambda$ $=0$, i.e., the shunting circuits are open. In the figures, $\omega_{0}=\sqrt{k_{\text {eff }} / m_{2}}$ is the local resonance frequency of the cantilever resonator system with the open circuits with $k_{e f f}=D_{b} / 3 l_{p}^{3}$. The first band gap is in the range of $\omega=\omega_{0} \sim 1.5 \omega_{0}$. Figure 2-10(b) shows the dispersion diagram and the first band gap of the elastic metamaterial plate when $\lambda=-0.83$, in which the effective modulus of the shunted piezoelectric patch increases in a positive value, therefore the bending stiffness of the cantilever beam is increased. The width of the band gap is almost not changed, however, the frequency range of the first band gap is shifted from the original $\left(\omega_{0} \sim 1.5 \omega_{0}\right)$ to $\left(1.13 \omega_{0} \sim 1.7 \omega_{0}\right)$. Figure 2-10(c) shows dispersion diagram and the first band gap of the elastic metamaterial plate when $\lambda=-0.842$, in which the effective stiffness of the shunted piezoelectric patch continuously increases in a positive value. The first band gap is shifted from the original band gap $\left(\omega_{0} \sim 1.5 \omega_{0}\right)$ to $\left(1.19 \omega_{0} \sim\right.$ $\left.1.8 \omega_{0}\right)$. However, if the effective stiffness of the shunted piezoelectric patch continuously increases to a larger value, the local resonance is hard to be induced in the cantilever beam in the low-frequency range because of the finite stiffness in the host medium. Figure 210(d) shows the band structure diagram of the elastic metamaterial plate when $\lambda=-0.898$, in which the effective modulus of the shunted piezoelectric patch becomes a negative value, and therefore the effective bending stiffness of the cantilever beam is decreased. Comparing the band diagram with the open circuit, it is found that the band gap is shifted 
from the original band gap $\left(\omega_{0} \sim 1.5 \omega_{0}\right)$ to $\left(0.62 \omega_{0} \sim 0.87 \omega_{0}\right)$. Figures 2-10(e) and 2-10(f) show the band structure diagram of the elastic metamaterial plate when $\lambda=-0.88$ and $\lambda=$ -0.8775 , respectively, in which the effective stiffness of the shunted piezoelectric patch is further reduced in a negative value. It is seen that the extremely low-frequency band gaps are located at the ranges of $\left(0.33 \omega_{0} \sim 0.42 \omega_{0}\right)$ and $\left(0.15 \omega_{0} \sim 0.19 \omega_{0}\right)$ for both cases. However, the width of the stop band becomes very narrow at the low-frequency cases for the bending wave. One important requirement to achieve the tunable band gap is to have the ability to adjust the value of the negative capacitance very accurately.
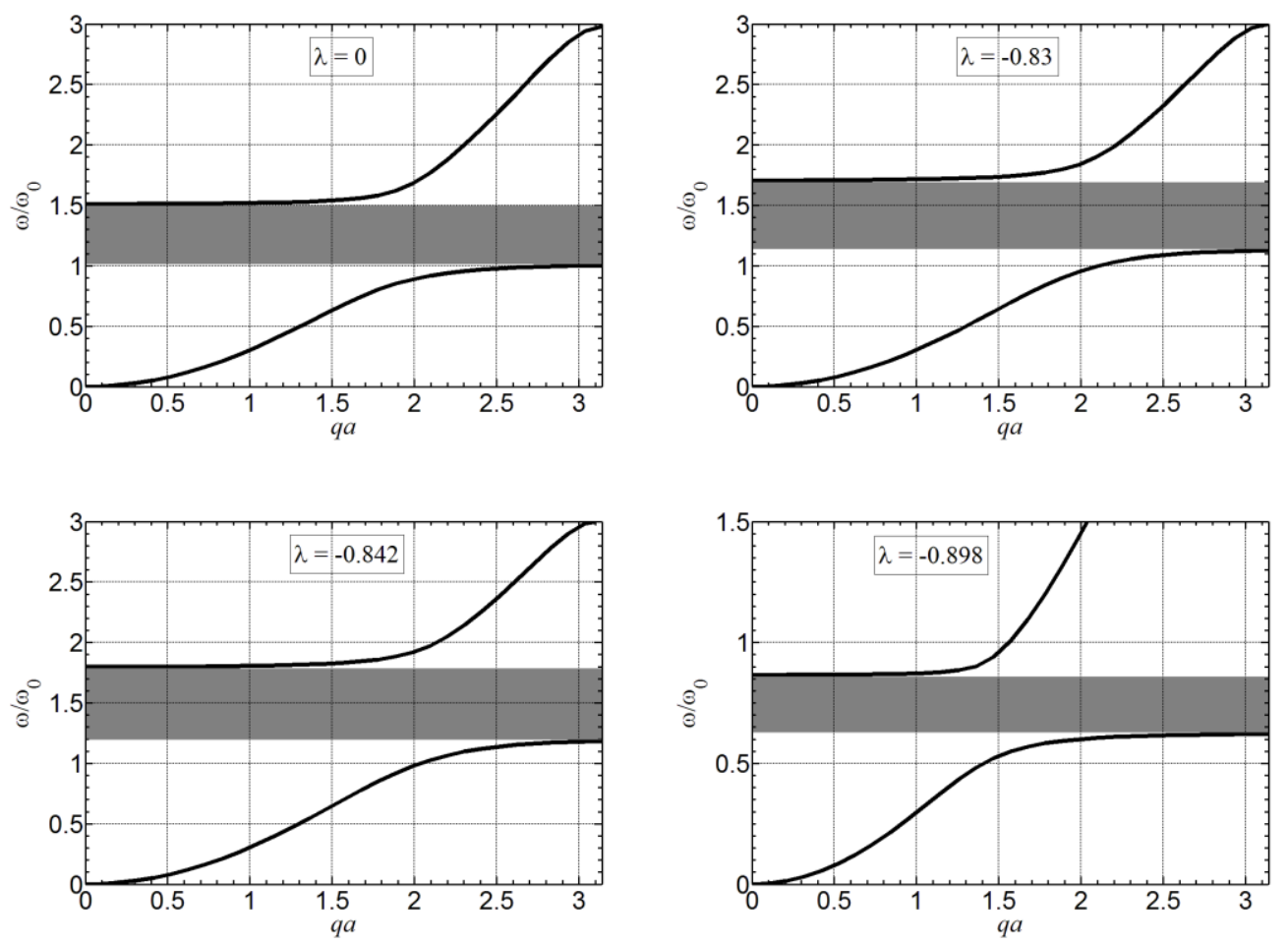

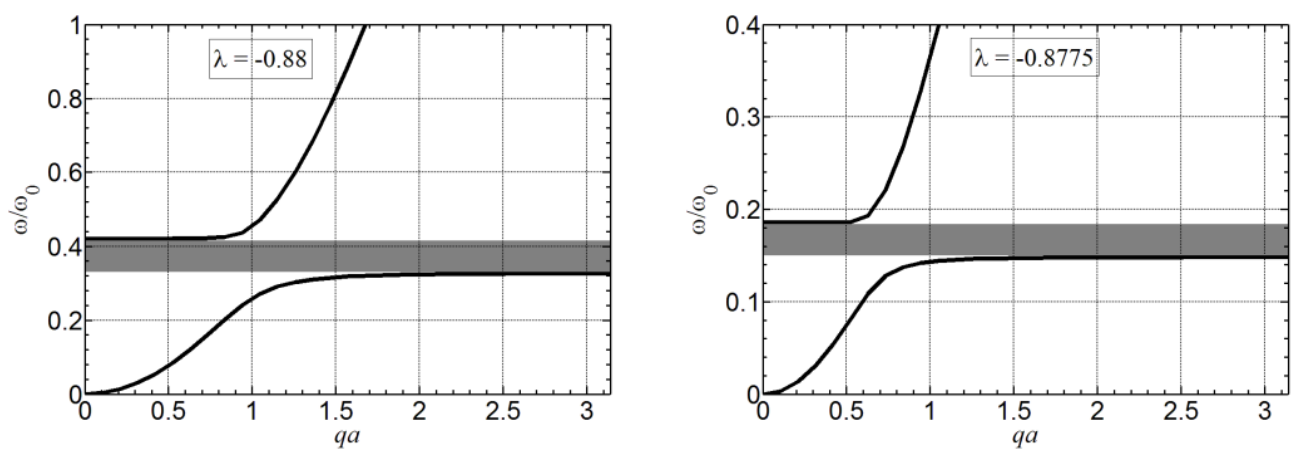

Figure 2-10 Band gap variation of the active elastic metamaterial plate with different

NCRs for out-of-plane bending wave

\subsection{Summary}

In this chapter, we first investigated dispersion curves and the band gap control in an active mass-in-mass lattice system. The unit cell of the lattice system consists of inner masses connected by active linear springs to represent negative capacitance piezoelectric shunting. It was demonstrated that the band gaps can be actively controlled and tuned by varying the effective spring constant through applying negative capacitance. Subsequently, this technique is implemented in the active elastic metamtaerial plate with periodically surface-bonded piezoelectric patches which are shunted with a negative capacitive circuit. It is demonstrated that the location and the range of induced band gap of the active elastic metamtaerial plate can be effectively tuned by using shunted piezoelectric patches with different NCRs, especially for extremely low-frequency cases. 


\section{Chapter 3 Experimental Demonstration of the Tunable Wave}

\section{Band Gaps}

\subsection{Introduction}

In Chapter 2, the negative capacitance (NC) piezoelectric shunting has implemented into the metastructures to actively tune the locally resonant (LR) frequency by modifying the stiffness of the resonant microstructure and therefore, controlling the band gap of the active elastic metamaterial (EMM). One advantage of a NC shunted circuit is the ability to continuously modify the resonant properties of the metastructures in a broad frequency range. However, there is still a lack of experimental validation of tunable bandgap behavior by considering shunting-piezo-elasto-dynamic effects in the metastructures. Fundamental elucidation of the circuit-feedback electromechanical coupling behavior of the active metastructures is therefore critical for future adaptive metamaterial designs.

In this chapter, a design for an adaptive EMM is proposed by integrating the shunted $\mathrm{NC}$ piezoelectric patches into the metastructure. The adaptive EMM is then manufactured and its experimental validation for tunable bandgap behavior is performed for the first time in a deep subwavelength scale without physical microstructural modifications. Finally, transient wave propagation tests of the adaptive metamaterial subjected to impact loads are conducted to illustrate their tunable wave mitigation abilities in real time. It can be concluded that the additional degrees of freedom offered by shunted piezoelectric materials can be used to shift the working frequency in the EMM and therefore their adaptive qualities can lead to qualitative improvement for passive metamaterials. 


\subsection{Design of the adaptive elastic metamaterials}

We first report a design for a new class of adaptive EMMs in which the electric circuits are used to control the subwavelength bandgap behavior in terms of frequency range without requiring microstructural modifications. The unit cell of the LR-based EMM is composed of an acrylic tube with periodically distributed I-type beams and lumped lead masses which are bonded to the central portion of the beam. The adaptive abilities of the EMM is then realized by integrating shunted piezoelectric patches on the opposite surface of the I-type beams. The schematic of the proposed adaptive EMM is shown in Fig. 3-1(a). The elastic modulus of the shunted piezoelectric patch and the effective bending stiffness of the composite beam can be tailored by adjusting different electric circuit parameters. Therefore, the interior LR frequency of the EMM can be electrically tailored. In the design, two NC circuits, namely softening and hardening circuits, are implemented to control the shunted piezoelectric patches to decrease and increase the effective bending stiffness of the beam, respectively. The softening circuit consists of a capacitor $C_{0}$, a potentiometer $R_{1}$, two resistors $R_{0}$ and $R_{2}$, and an operational amplifier which can be theoretically operated with the stability condition being negative equivalent capacitances $C_{N}>C_{p}$. For a second case, a resistor $R_{0}$ in the hardening circuit is replaced by a potentiometer in order to tune and satisfy the stability condition $C_{N}<C_{p}$.

To characterize the dynamic properties and provide an underlying physical interpretation of the adaptive metamaterial, a multi-physics effective model should be developed to capture the electro-mechanical coupling effect. However, due to the complex microstructure geometry in the unit cell, it is very difficult to directly derive an analytical model and therefore, a numerical-based multi-physics effective model is utilized to 
determine the effective properties which are implemented in COMSOL. The effective mass density of the adaptive metamaterial can be calculated based on the macroscopic resultant force and acceleration of the subwavelength metamaterial unit cell which can be obtained by averaging the local quantities on the external boundary of the unit cell in the multiphysics finite element model. Figure 3-1(c) shows the numerically calculated tunable effective mass densities of the EMM in function of both the normalized frequency $\omega^{*}$ and the negative capacitance ratio $\lambda$ of the NC circuit, where $\rho_{\text {eff }}^{*}, \omega^{*}$ and $\lambda$ are defined as $\rho_{\text {eff }}^{*}=\rho_{\text {eff }} / \rho_{0}, \quad \omega^{*}=\omega / \omega_{0}$ and $\lambda=C_{N} / C_{p}$, respectively. $\rho_{0}$ is the static effective density, $\omega_{0}$ is the LR angular frequency of the EMM's unit cell with an open circuit where $C_{p}$ is the capacitance of the piezoelectric patch with the open circuit. It can be found that the frequency range of the negative effective mass density shifts to a lower frequency range when $\lambda$ approaches to -1 from negative infinity (softening circuits) while it shifts to a higher frequency range when $\lambda$ approaches to -1 from zero (hardening circuits). Physically, the bandgap of the EMM can be electrically tuned at almost any desired frequency range by adjusting the $\mathrm{NC}$ values of the shunted piezoelectric patches without any change to the microstructures geometry. The tunability of the EMM provides a great potential for the design of a gradient-index EMM for applications in elastic wave steering and even elastic wave cloaking. However, in a realistic scenario, system instability due to the NC circuits and the electric damping of the piezoelectric patch can significantly reduce the tunable frequency range of the adaptive EMM, which will be discussed in the experimental validation. 


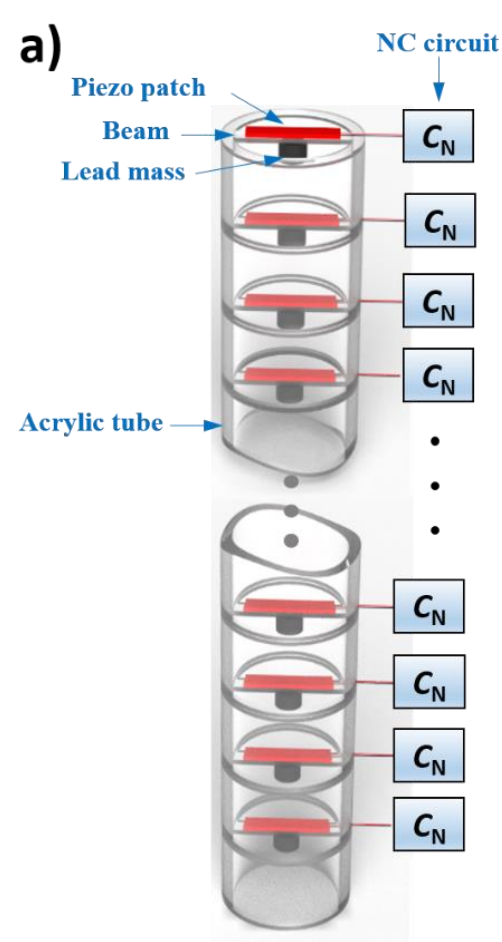

b)
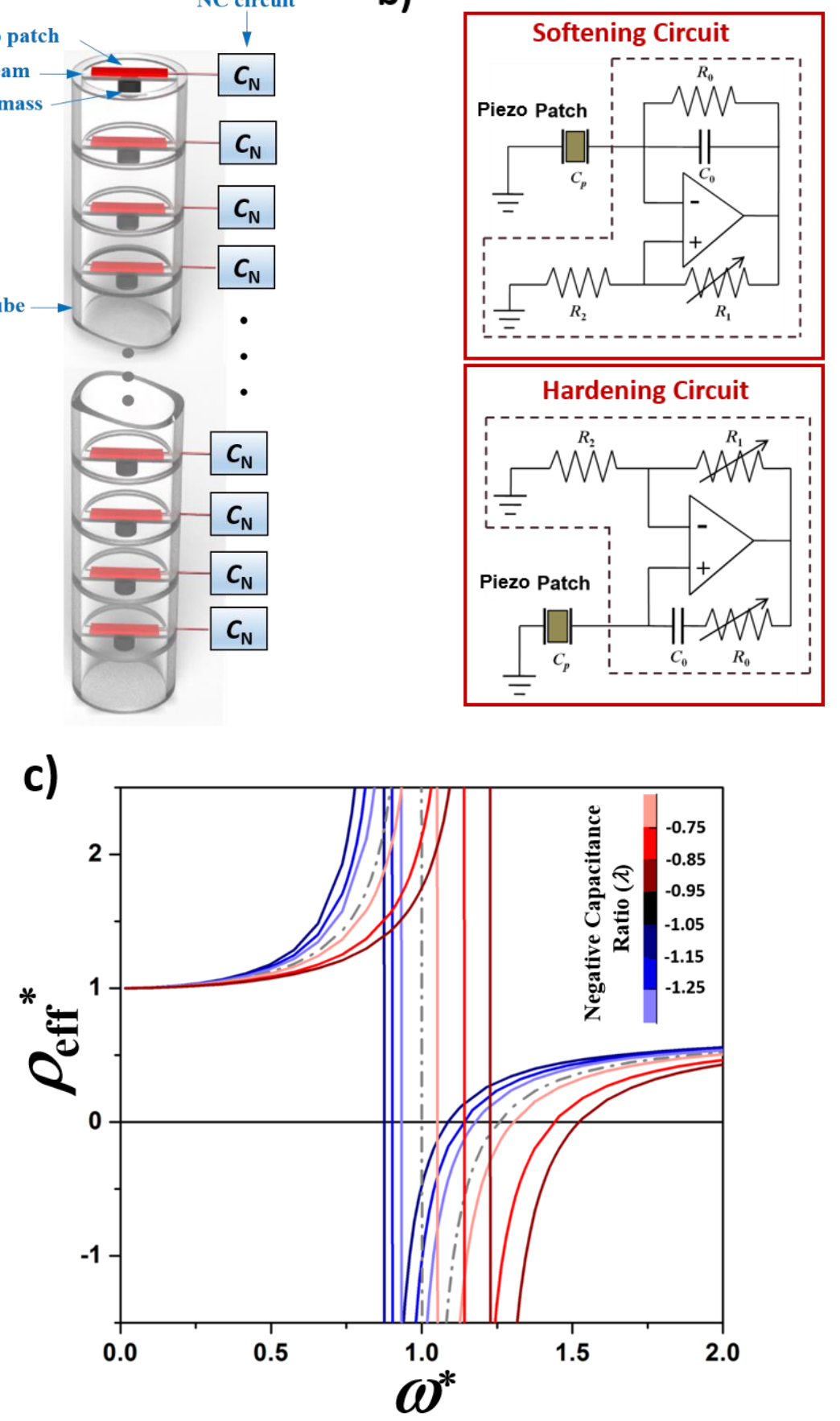

Figure 3-1 (a) The schematic of the adaptive EMM; (b) Softening and hardening NC circuits; (c) Effective mass densities of the adaptive EMM as a function of both normalized frequency and negative capacitance ratio 


\subsection{Experimental testing}

Dynamic experimental tests are conducted to characterize and validate the dynamic tunability of the adaptive EMM specimen. Figure 3-2(a) shows the fabricated adaptive EMM unit cell. Harmonic vibration tests are first performed on the unit cell by using a vibrational shaker (LDS V203). The NC circuit (either softening or hardening) is connected to the piezoelectric patch of the unit cell sample and powdered by a DC power supply (Instek SPD-3606). By changing the value of the potentiometer $R_{1}$ in the NC circuit, as shown in Fig. 3-1(b), the value of $\lambda$ varies in a range close to -1, and the resonant frequency of the unit cell can be tuned electrically. Figure 3-2(b) demonstrates the measured voltage signals from the piezoelectric patch when a vibration frequency sweep is conducted. It can be found that the resonant frequency of the adaptive unit cell can be manipulated in a wide frequency range, and the tunability of the resonant frequency, which is defined as $\left(\omega_{\max }^{*}-\right.$ $\left.\omega_{\min }^{*}\right)$, as high as $45 \%$ can be achieved, where $\omega_{\max }^{*}$ and $\omega_{\min }^{*}$ are the highest and lowest normalized resonant frequencies, respectively. Figure 3-2(c) shows the comparison between the measured results and those obtained from the numerical prediction using the multi-physics numerical model. In the figure, the circuits represent the measured results and the solid curves represent the numerically calculated resonant frequencies of the proposed adaptive unit cell. Very good agreement between the experimental measurement and numerical prediction can be observed, which indicates the feasibility and accuracy of the developed multi-physics numerical model. It should be mentioned that the wavelength in the tunable resonant frequency range is about 30 times larger than the length of the adaptive unit cell. 
a)

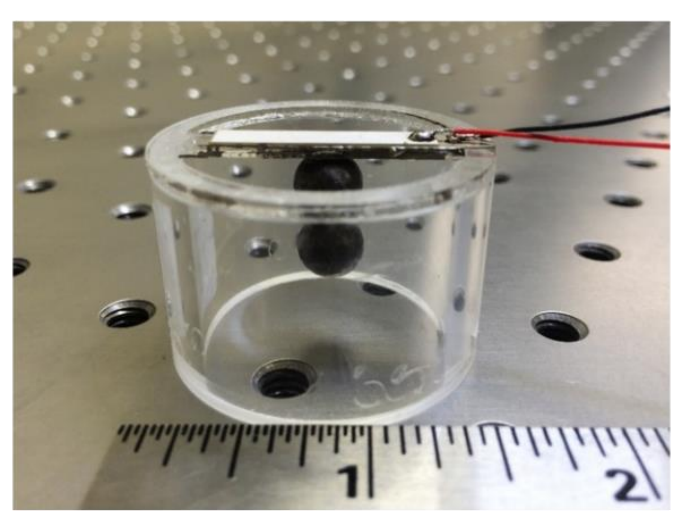

b)

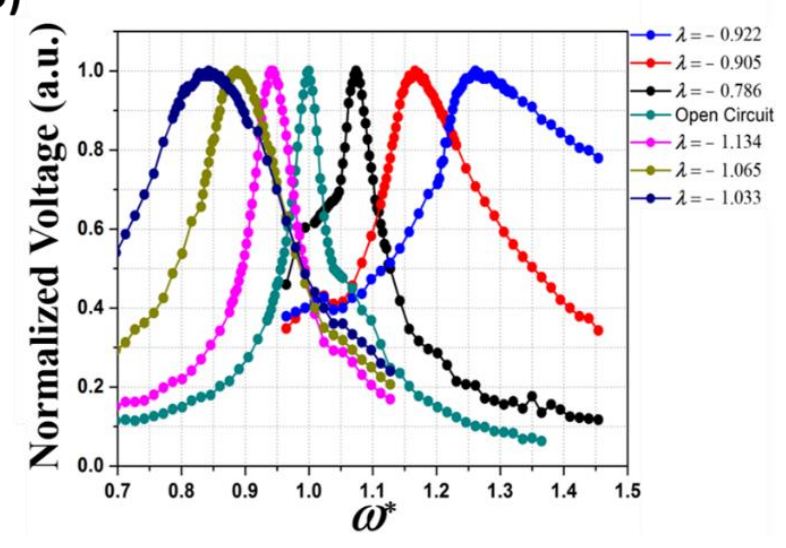

c)

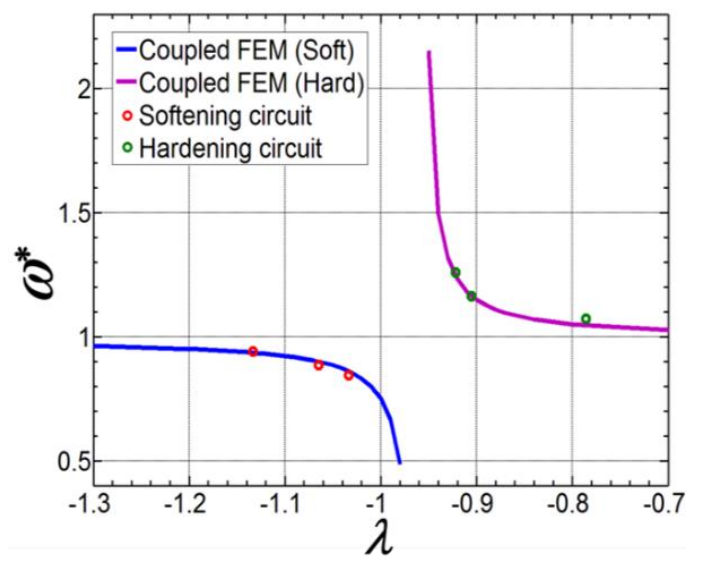

Figure 3-2 (a) Photos of the fabricated adaptive unit cell; (b) Measured tunable resonant frequency of the adaptive unit cell when different negative capacitance ratios were applied; (c) Comparison of the resonant frequencies of the unit cell between the experimental testing and numerical simulation

To quantitatively demonstrate tunable bandgap behavior, an adaptive EMM specimen is formed by assembling nine adaptive unit cells controlled by NC circuits. A tenth cell composed of an empty acrylic tube and circular cap is placed on the top of the EMM for accelerometer installment. In the experimental testing, a white noise excitation signal is first produced by a dynamic analyzer (Bruel \& Kjaer PHOTON+) and then amplified by a 
power amplifier (LDS PA25E) which drives the shaker (LDS V203) to generate an excitation with frequencies from 0 to $1200 \mathrm{~Hz}$. Finally, an accelerometer (Bruel \& Kjaer Accelerometer Type 4516) was attached to the top end of the EMM and connected to the dynamic signal analyzer for capturing the transmitted signals. Figure 3-3(a) shows the experimentally measured frequency response functions (FRFs) of the adaptive EMM connected to different NC circuits. For comparison, FRFs in the pure acrylic tube and the adaptive EMM with the open circuit are also plotted as dot and dash curves, respectively. In the figure, obvious transmission dips can be observed at different frequency ranges for the adaptive EMM connected with different NC circuits, which confirms the tunable wave attenuation ability of the adaptive EMM sample. Furthermore, the transmission dips can be electronically shifted to different frequency ranges by adjusting circuits (with different $\lambda$ ) without requiring any microstructural modifications. Specifically, the wave attenuation range of the adaptive EMM shifts to the lower frequency range for the EMM with the softening circuits $(\lambda>-1)$ and goes to a higher frequency range for the EMM with the hardening circuits $(0<\lambda<-1)$. To interpret the adaptive wave attenuation mechanism, the tunable effective mass density of the proposed EMM is calculated using the multi-physics numerical model, as shown in Fig. 3-3(b). It is very interesting to note that the tunable bandgap behavior of the EMM can almost be predicted from the frequency ranges of the tunable negative effective mass density, which indicates that the tunable bandgap behavior is fully controlled by the out-of-phase motions of the EMM and the NC circuits. The minor difference can be attributed to factors from the electric damping and interior loss factor of the piezoelectric patches, which is not considered in the numerical modeling. 


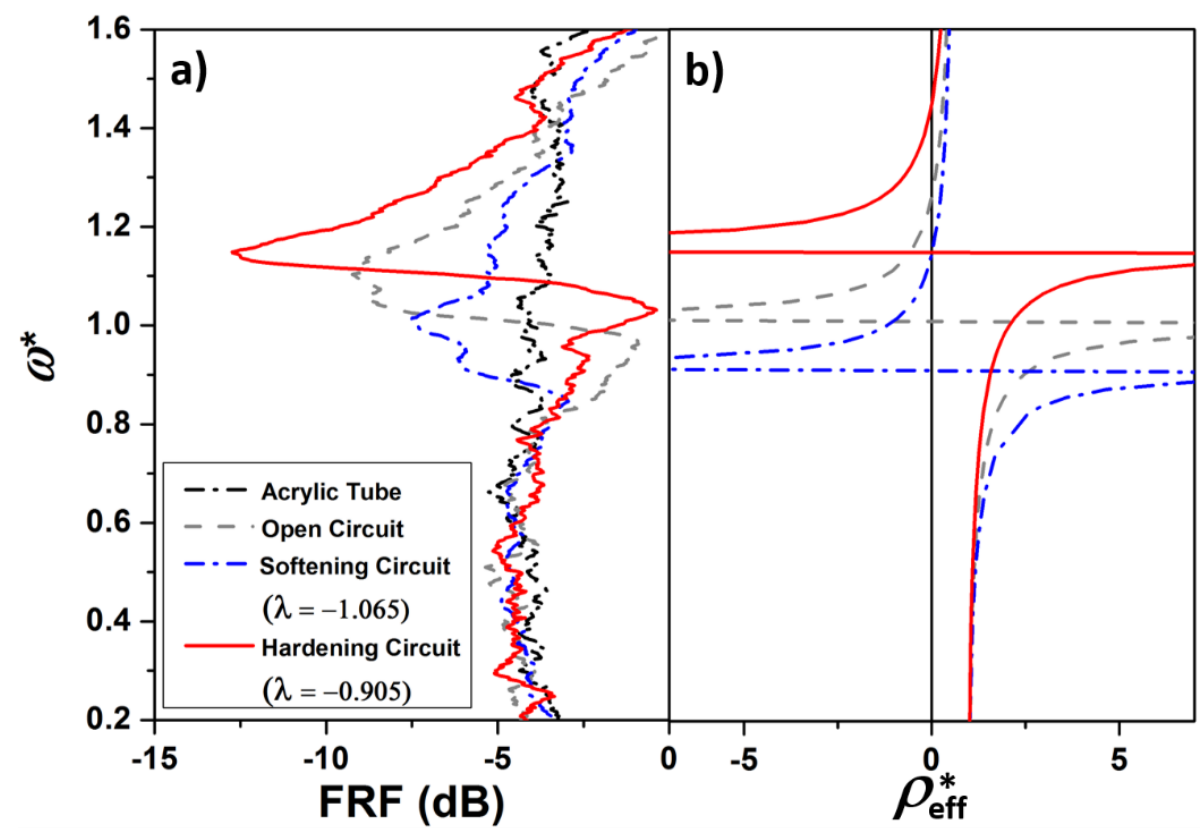

Figure 3-3 (a) Experimentally measured frequency response functions of the adaptive EMMs connected with different NC circuits; (b) Numerically calculated effective mass densities with different negative capacitance ratios

Thanks to advantages from the subwavelength-scale size and the high wave attenuation ability, metamaterials were suggested for applications in blast wave mitigation. To demonstrate the unique feature of the adaptive EMM, especially in terms of tunable transient wave mitigation, we will further experimentally investigate the transient wave propagation in the adaptive EMM. In the transient wave experiment, a striker, which is made of an aluminum block with a cross section slightly larger than that of the adaptive EMM, is attached to an impact pendulum with adjustable height and impact angle $\theta$ to provide impact wave excitation. Consistency of the excitation signals is ensured by precisely controlling the release angle of the impact pendulum, for example $\theta=15^{\circ}$. A steel enclosure disk is glued to the left end of the adaptive EMM specimen to ensure a short 
incident pulse and mimic the real-world impact excitation. Two miniature piezoelectric sensors (P-6.36mm-0.33mm-850, WFB, APC International, LTD) are bonded before and after the adaptive EMM for measuring the incident and transmitted waves, respectively. A long pure acrylic tube is attached to the right end of the adaptive EMM specimen to separate the reflected wave from the transmitted wave. The entire testing sample is suspended from rigid supports using thin, lightweight strings so as to obtain a free-free boundary condition.

Figure 3-4 shows the experimentally measured incident and transmitted wave signals in terms of the electric voltages in both time and frequency domains. In the experiment, three different circuits which are open, softening $(\lambda=-0.922)$ and hardening $(\lambda=$ $-1.065)$ circuits are used to actively control the wave properties of the adaptive EMMs. From the time domain results, as shown in Figs. 3-4(a)-(c), it can be observed that the transmitted pulse is very complex to directly interpret and only an obvious decrease in amplitudes can be observed, which indicates that the incident impact energy has been efficiently attenuated by the adaptive EMM. Moreover, from the frequency-domain results, as shown in Figs. 3-4(d)-(f), wave attenuation dips at different frequencies can be clearly observed with different shunted electric circuits. Specifically, it is found that the adaptive EMM with an open circuit has a maximum transmission dip at $680 \mathrm{~Hz}$ while adaptive EMMs with softening $(\lambda=-1.065)$ and hardening circuits $(\lambda=-0.922)$ have maximum transmission dips at $530 \mathrm{~Hz}$ and $1200 \mathrm{~Hz}$, respectively. It should be mentioned that the wave mitigation ability of the adaptive EMM could be significantly increased by adding more EMM unit cells. It can also be conceived that in conjunction with the proper control system, the tunable wave mitigation could be operated in real time because of the quick response time of the shunted electric circuits. 

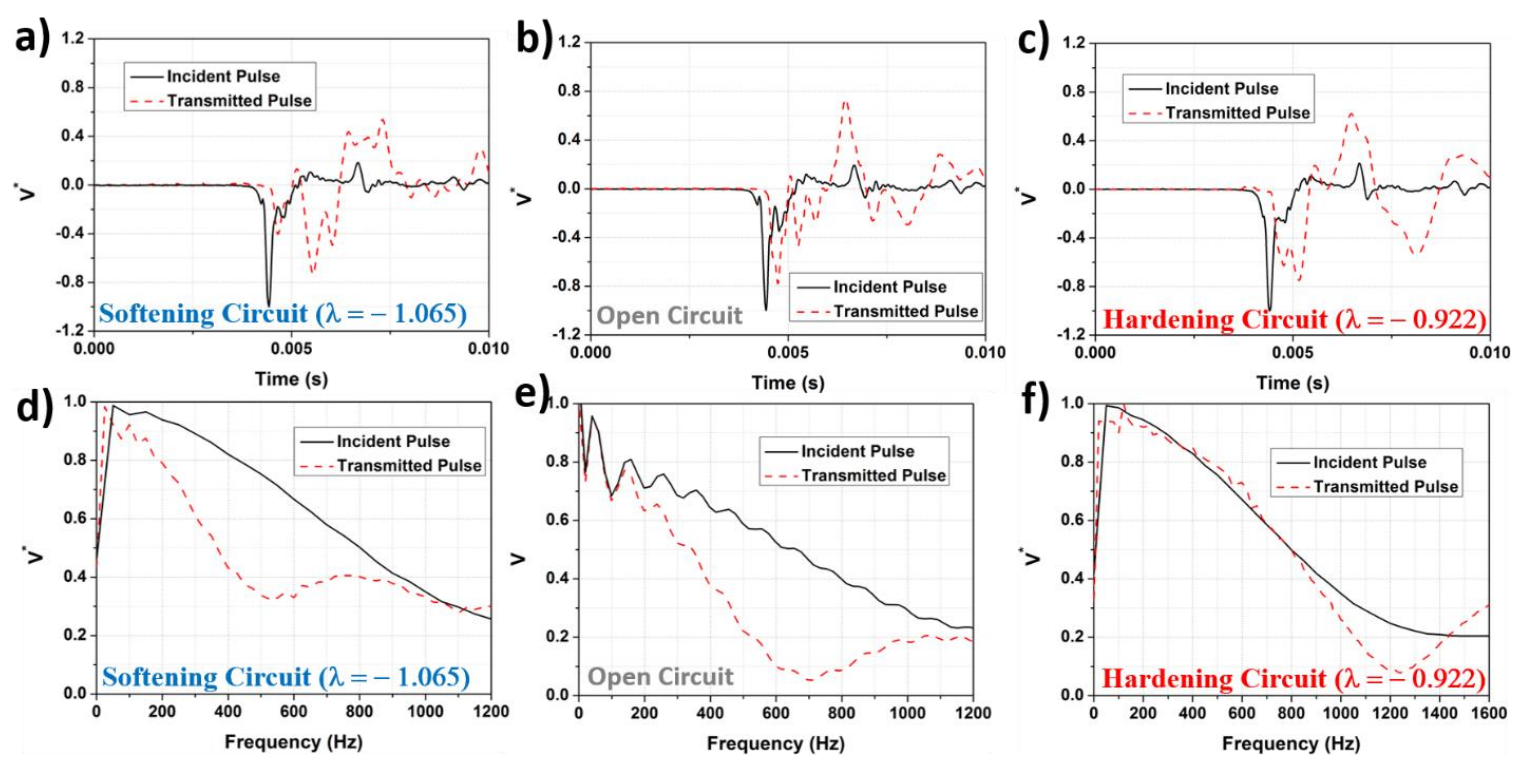

Figure 3-4 The time-domain incident and transmitted signals for adaptive EMM with (a) softening circuit $(\lambda=-1.065)$, (b) open circuit and (c) hardening circuit $(\lambda=-0.922)$. The frequency-domain incident and transmitted pulses for adaptive metamaterial with (d) softening circuit $(\lambda=-1.065)$, (e) open circuit and (f) hardening circuit $(\lambda=-0.922)$

\subsection{Conclusions}

In this chapter, we experimentally investigate the dynamic behavior of an adaptive EMM which is actively controlled by negative capacitance piezoelectric shunting. Based on the multi-physics numerical model developed, the quantitative relationship between the effective mass density of the metamaterial and shunted electric circuits is established. By changing the values of the potentiometer in the negative capacitance circuits, the resonant frequency of the EMM and a tunable bandgap capacity as high as $45 \%$ are physically realized by using both shunted hardening and softening circuits. Finally, transient impact wave propagation tests are conducted and the obtained results demonstrate the promising possibilities to dynamically mitigate and filter different wave frequencies in real time. 


\section{Chapter 4 An Adaptive Metamaterial Beam for Extremely}

\section{Broadband Wave Attenuations}

\subsection{Introduction}

In Chapter 3, we designed an adaptive elastic metamaterial, where harmonic vibration testing as well as transient wave propagation tests were conducted on the fabricated metamaterial sample to illustrate their tunable vibration suspension and elastic wave attenuation abilities. However, actively controlled bandgaps with position and width variations are still narrow in real-time, which eventually cannot be used for broadband incident loadings.

In this chapter, we consider a novel approach to significantly broaden the subwavelength band gaps of an adaptive metamaterial beam to extremely low or high frequency regions based on periodic arrays of local resonators with hybrid circuit shunted piezoelectric stacks. The negative capacitance and negative inductance shunting circuits are connected to the same individual piezoelectric stack in series or in parallel. The complex band structure of the infinite periodic structure is predicted by the transfer matrix (TM) method, which is compared with the frequency response function of a finite structure calculated by a multi-physics finite element (FE) method. Our analytical and numerical results demonstrate that hybrid shunting circuits can create super-wide band gaps to achieve high-pass and low-pass wave filtering by appropriately selecting the values of negative capacitance and negative inductance to adaptively tune the effective modulus of piezoelectric stacks. This design is very significant for the practical applications of lowfrequency sound and structural vibration/wave attenuations in engineering. 


\subsection{The adaptive metamaterial beam and modeling methods}

\subsubsection{Microstructures of the adaptive metamaterial beam with hybrid shunting circuits}

Figure 4-1(a) shows the schematic of the proposed adaptive metamaterial beam design, which is comprised of a host beam with periodically surface bonded piezoelectric stacks with cylindrical proof masses on the stacks' top surfaces. Each of the piezoelectric stacks is made of several stacked rectangular piezoelectric plates for strong electromechanical coupling and are individually shunted with uniform hybrid circuits for modulus control. The employment of piezoelectric stacks instead of piezoelectric bars in the proposed microstructure can significantly enlarge the capacitance of the piezoelectric element and reduce the high voltage requirement in the modulus control, which offers great benefits in the metamaterial applications. Specifically, two kinds of hybrid circuits, TYPE I and TYPE II circuits (shown in the right hand side of Fig. 4-1(a)), are considered in the study for broadband low and high frequency wave attenuations, respectively. The TYPE I circuit is integrated with negative capacitance $C_{1}$ and negative inductance $L_{1}$ connected in series. The TYPE II circuit is constructed with a negative capacitance $C_{2}$ and negative inductance $L_{2}$ connected in parallel. Both of the two kinds of hybrid circuits can be achieved with analog negative impedance circuits or digital synthetic impedance circuits. As also shown in Fig. 4-1(a), the side length and height of piezoelectric stacks as well as diameter and height of the cylindrical masses are denoted as $a_{p}, h_{p}, d_{m}$ and $h_{m}$, respectively. The thickness

of the host plate and lattice constant of the periodic metamaterial are represented by $h_{b}$ and $L_{b}$, respectively. The width of the metamaterial beam is selected to be the same as the lattice constant. The main creativity of the current microstructure design is that the dimension of 
the piezoelectric stack with a relatively large height to width ratio is much smaller than the dimension of the proof mass. Therefore, the dominated deformation of the piezoelectric stack will be along the $z$ direction as an effective mechanical connectivity (stress-strain correlation along the longitudinal direction of the object between the host beam and the attached resonator, which is quantitatively equivalent to the effective modulus of the connected piezoelectric stack), when the metamaterial beam is subjected to a flexural wave. As a result, the in-plane and out-of-plane coupling motions of the local resonators can be significantly minimized.

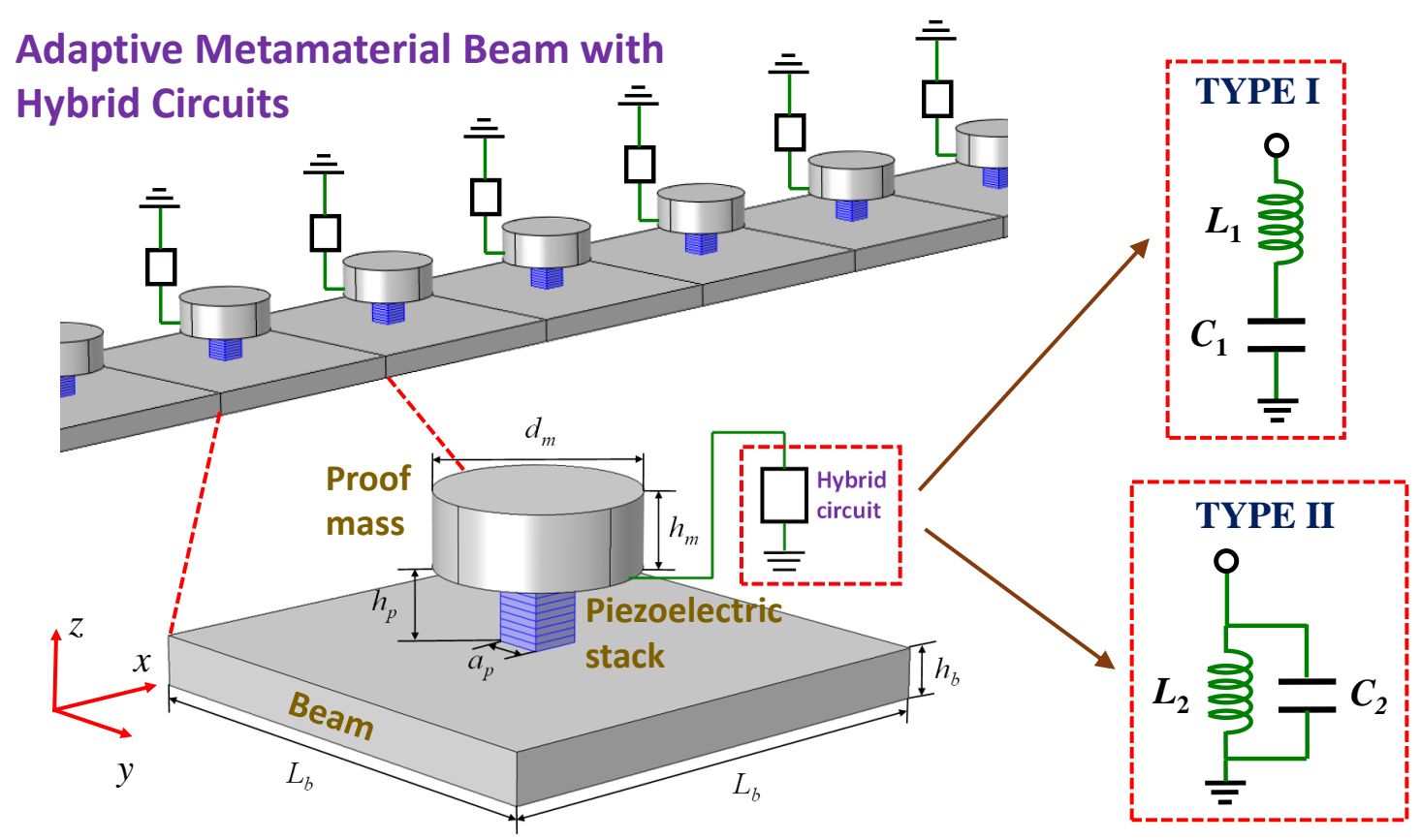

(a)

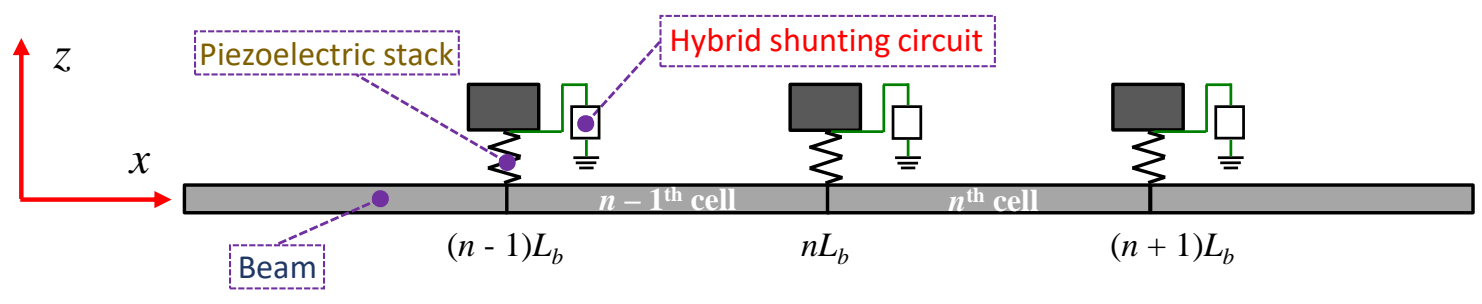

(b) 
Figure 4-1 (a) Schematic of the adaptive metamaterial beam with piezoelectric stacks shunted by hybrid negative capacitance and negative inductance circuits; (b) Schematic of the $2 \mathrm{D}$ theoretical model of the adaptive metamaterial beam

\subsubsection{Modulus tuning of piezoelectric stacks shunted with hybrid circuits}

According to the model by Hagood [50], both the storage and loss modulus of piezoelectric materials can be controlled by external electrical shunting networks. Based on this concept, the variation of the stiffness of piezoelectric stacks shunted with hybrid circuits is quantitatively characterized. Different from previous studies, which considered frequency independent modulus variations by using negative capacitance, this study focuses on frequency dependent modulus alterations in order to achieve extremely broadband wave control. Here, we consider a piezoelectric stack composed with $N$ number of layers of piezoelectric plates with the same geometric dimensions. The polarization directions of each of the two adjacent individual plates are opposite in the stack design (being positive and negative $z$ directions or vice versa). In this study, the boundary effects from the interfaces between the stacks, host beam and proof masses are ignored, due to the large height to width ratio of the piezoelectric stack used in the adaptive metamaterial design. The longitudinal deformation along the $z$-direction is assumed to be uniform within each of the stacks. Since all four side surfaces of the stack are traction and charge free, the in-plane stresses and electric fields in each of the individual piezoelectric plates are neglected. Based on these assumptions, the 3D piezoelectric constitutive equations can be reduced to two 1D equations for the piezoelectric plates with polarization directions along positive $z$ direction as 


$$
\begin{aligned}
& S_{33}=d_{33} E_{3}+s_{33}^{E} T_{33} \\
& D_{3}=\varepsilon_{33}^{T} E_{3}+d_{33} T_{33}
\end{aligned}
$$

where $S_{33}, T_{33}, D_{3}$ and $E_{3}$ denote the stress, strain, electric displacement and electric field along the $z$ direction, respectively, and $s_{33}^{E}, d_{33}$ and $\varepsilon_{33}^{T}$ represent the elastic compliance at constant electric field, piezoelectric coefficient and dielectric coefficient at constant stress of the piezoelectric material, respectively. By properly considering the electrical boundary conditions due to the shunting impedance $Z_{s h}$, the dynamic effective modulus of the piezoelectric stack can be obtained as [44]

$$
E_{p}^{e f f}=\frac{E_{p}^{E}}{1-\frac{k_{33}^{2}}{1+1 /\left(i \omega Z_{S h} C_{p}^{T}\right)}}
$$

where $E_{p}^{E}=\frac{1}{s_{33}^{E}}, k_{33}=\sqrt{\frac{d_{33_{33}^{2}}^{E}}{\varepsilon_{33}^{T}}}$ and $C_{p}^{T}=\frac{N^{2} a_{p}^{2} \varepsilon_{33}^{T}}{h_{p}}$ are the short circuit modulus, electromechanical coupling coefficient and the capacitance of the piezoelectric stack, respectively. According to Eq. (4.2), if the shunting impedance is not purely capacitive, the final effective modulus will be in function of both the shunting impedance and the working frequency. Specifically, for the TYPE I and TYPE II circuits, the shunting impedances can be expressed, respectively, as

$$
\begin{aligned}
& Z_{s h}^{(1)}=\frac{1}{i \omega C_{p}^{T} \lambda_{1}}-\frac{i \omega}{\omega_{s 1}^{2} C_{p}^{T}} \\
& Z_{s h}^{(2)}=\frac{1}{i \omega C_{p}^{T} \lambda_{2}-\frac{\omega_{s 2}^{2} C_{p}^{T}}{i \omega}}
\end{aligned}
$$


where $\lambda_{i}=\frac{C_{i}}{C_{p}^{T}}$ and $\omega_{s i}^{2}=\frac{1}{\left|L_{i}\right| C_{p}^{T}}$ with $i$ being 1 or 2 . Thus, by substituting Eqs. (4.3a) and (4.3b) into Eq. (4.2), the equivalent modulus due to these two kinds of shunting circuits can be obtained.

\subsubsection{Dispersion relations of the adaptive metamaterial beam}

In order to characterize the unique wave attenuation properties of the adaptive metamaterial beam, an analytical model based on TM method is developed to consider effects from hybrid shunting circuits, which is shown in Fig 4-1(b). In the model, a plane stress assumption is used for the metamaterial beam structure. For simplicity, the piezoelectric stacks are represented by linear springs with tunable stiffness $k_{p}=\frac{E_{p}^{e f f} a_{p}^{2}}{h_{p}}$ controlled by the shunting circuits. The proof masses' rotational and $x$ direction translational motions are ignored in the model. Based on the Timoshenko beam theory, the governing equation for the host beam can be written as [44]

$$
\frac{E_{b} I_{b}}{\rho_{b} S_{b}} \frac{\partial^{4} w}{\partial x^{4}}-\frac{I_{b}}{S_{b}}\left(1+\frac{E_{b}}{\kappa_{b} G_{b}}\right) \frac{\partial^{4} w}{\partial x^{2} \partial t^{2}}+\frac{\partial^{2} w}{\partial t^{2}}+\frac{\rho_{b} I_{b}}{\kappa_{b} G_{b} S_{b}} \frac{\partial^{4} w}{\partial t^{4}}=0
$$

where $w$ denotes the out-of-plane displacement field on the neutral plane of the host beam. $E_{b}, G_{b}, \rho_{b}, I_{b}, S_{b}$, and $\kappa_{b}$ are the Young's modulus, shear modulus, mass density, area moment of inertia, area of cross section and Timoshenko shear coefficient of the host beam, respectively. In our analysis, we consider a harmonic solution to Eq. (4.4) as $w(x, t)=$ $W(x) \exp (i \omega t)$, and the harmonic time terms are suppressed in the following equations. For the $n$-th unit cell, the general solution can be expressed as [44]

$$
W_{n}\left(x_{n}\right)=A_{n} e^{\lambda_{1} x_{n}}+B_{n} e^{\lambda_{2} x_{n}}+C_{n} e_{n}^{\lambda_{3} x_{n}}+D_{n} e^{\lambda_{4} x_{n}}
$$


where $\quad x_{n}=x-n L_{b} \quad, \quad n L_{b} \leq x \leq(n+1) L_{b} \quad, \quad \lambda_{1,2,3,4}= \pm \sqrt{\frac{\alpha \pm \sqrt{\alpha^{2}+4 \beta}}{2}} \quad$ with $\alpha=-\frac{\rho_{b} \omega^{2}}{E_{b}}-\frac{\rho_{b} \omega^{2}}{\kappa_{b} G_{b}}$ and $\beta=\frac{\rho_{b} S_{b} \omega^{2}}{E_{b} I_{b}}-\frac{\rho_{b}^{2} \omega^{4}}{\kappa_{b} G_{b} E_{b}}$. The out-of-plane point force on the interface between the $n$-th and $(n+1)$-th unit cell caused by the tunable resonator can be expressed as [44]

$F_{n}=\frac{-\omega^{2} m_{r} k_{p} W_{n}(0)}{-\omega^{2} m_{r}+k_{p}}$

where $m_{r}$ represents the weight of the cylindrical proof mass. Different from the previous TM method used in metamaterial beams, which considered boundary continuity conditions based on Euler beam assumptions, the currently developed TM method considers boundary continuity conditions based on Timoshenko beam theory in consistent with the governing equation. This treatment is necessary, when the thickness of the host beam becomes larger and the resonant band gap is close to the Bragg band gap. Thus, the continuity conditions of the displacement, total rotation angle of the cross-section, bending moment and shear force at the point, $x=n L_{b}$, require

$$
\begin{aligned}
& W_{n}(0)=W_{n-1}\left(L_{b}\right) \\
& \psi_{n}(0)=\psi_{n-1}\left(L_{b}\right) \\
& E_{b} I_{b} \frac{\partial \psi_{n}(0)}{\partial x}=E_{b} I_{b} \frac{\partial \psi_{n-1}\left(L_{b}\right)}{\partial x} \\
& \kappa_{b} G_{b} S_{b}\left(\frac{\partial W_{n}(0)}{\partial x}-\psi_{n}(0)\right)=\kappa_{b} G_{b} S_{b}\left(\frac{\partial W_{n-1}\left(L_{b}\right)}{\partial x}-\psi_{n-1}\left(L_{b}\right)\right)+F_{n}
\end{aligned}
$$


where $\psi=\frac{d W}{d x}+\gamma$, where $\gamma$ is the shear angle. Substituting Eqs. (4.5) and (4.6) into Eq.

(4.7), one can obtain

$$
\mathbf{K} \mathbf{A}_{n}=\mathbf{H} \mathbf{A}_{n-1}
$$

where

$$
\begin{aligned}
\mathbf{K} & =\left[\begin{array}{cccc}
1 & 1 & 1 & 1 \\
a \lambda_{1}+b \lambda_{1}^{3} & a \lambda_{2}+b \lambda_{2}^{3} & a \lambda_{3}+b \lambda_{3}^{3} & a \lambda_{4}+b \lambda_{4}^{3} \\
a \lambda_{1}^{2}+b \lambda_{1}^{4} & a \lambda_{2}^{2}+b \lambda_{2}^{4} & a \lambda_{3}^{2}+b \lambda_{3}^{4} & a \lambda_{4}^{2}+b \lambda_{4}^{4} \\
\lambda_{1}-\frac{F_{n}}{\kappa_{b} G_{b} S_{b}} & \lambda_{2}-\frac{F_{n}}{\kappa_{b} G_{b} S_{b}} & \lambda_{3}-\frac{F_{n}}{\kappa_{b} G_{b} S_{b}} & \lambda_{4}-\frac{F_{n}}{\kappa_{b} G_{b} S_{b}}
\end{array}\right], \\
\mathbf{H} & =\left[\begin{array}{cccc}
e^{\lambda_{1} L_{b}} & e^{\lambda_{2} L_{b}} & e^{\lambda_{3} L_{b}} & e^{\lambda_{4} L_{b}} \\
\left(a \lambda_{1}+b \lambda_{1}^{3}\right) e^{\lambda_{1} L_{b}} & \left(a \lambda_{2}+b \lambda_{2}^{3}\right) e^{\lambda_{2} L_{b}} & \left(a \lambda_{3}+b \lambda_{3}^{3}\right) e^{\lambda_{3} L_{b}} & \left(a \lambda_{4}+b \lambda_{4}^{3}\right) e^{\lambda_{4} L_{b}} \\
\left(a \lambda_{1}^{2}+b \lambda_{1}^{4}\right) e^{\lambda_{1} L_{b}} & \left(a \lambda_{2}^{2}+b \lambda_{2}^{4}\right) e^{\lambda_{2} L_{b}} & \left(a \lambda_{3}^{2}+b \lambda_{3}^{4}\right) e^{\lambda_{3} L_{b}} & \left(a \lambda_{4}^{2}+b \lambda_{4}^{4}\right) e^{\lambda_{4} L_{b}} \\
\lambda_{1} e^{\lambda_{1} L_{b}} & \lambda_{2} e^{\lambda_{2} L_{b}} & \lambda_{3} e^{\lambda_{3} L_{b}} & \lambda_{4} e^{\lambda_{4} L_{b}}
\end{array}\right], \\
\mathbf{A}_{n} & =\left[\begin{array}{lllll}
A_{n} & B_{n} & C_{n} & D_{n}
\end{array}\right]^{T},
\end{aligned}
$$

with

$$
\begin{aligned}
& a=\frac{\kappa_{b} G_{b} E_{b}+\frac{\rho_{b} \omega^{2} E_{b} I_{b}}{\kappa_{b} G_{b}}}{\kappa_{b} G_{b} E_{b}-\rho_{b} \omega^{2} I_{b}}, \\
& b=\frac{E_{b} I_{b}}{\kappa_{b} G_{b} E_{b}-\rho_{b} \omega^{2} I_{b}} .
\end{aligned}
$$

Due to the periodicity of the metamaterial beam in the $x$-direction, the Bloch theorem guarantees 


$$
\mathbf{A}_{n}=e^{i k_{x} L_{b}} \mathbf{A}_{n-1}
$$

where $k_{x}$ is the wave vector along the $x$-direction. By inserting Eq. (4.9) into Eq. (4.8), a standard eigenvalue problem is yielded as

$$
\left|\mathbf{K}^{-1} \mathbf{H}-e^{i k_{x} L_{b}} \mathbf{I}\right|=0
$$

where I is the $4 \times 4$ identity matrix. Finally, Eq. (4.10) illustrates the dispersion relations of the adaptive metamaterial beam with hybrid shunting circuits. Based on the given frequencies, the complex wavenumbers are determined, from which the wave propagation properties are characterized.

\subsection{Results and discussion}

In this section, the complex band structure of the metamaterial beam with various effective mechanical connectivities (spring stiffness) is characterized first. Based on the properties of the band gap behaviors observed, two kinds of hybrid shunting circuits are designed to electronically realize the physical properties. The performance of the adaptive metamaterial beam utilizing these hybrid circuits is then examined by conducting multiphysics numerical simulations on a finite metamaterial beam to demonstrate extremely broadband attenuation capabilities.

\subsubsection{Complex band structures of the metamaterial beam}

As an illustrative example, we select steel for the material of the host beam and cylindrical proof masses. The piezoelectric stacks are made of PMN-33\%PT single crystals, which usually have larger piezoelectric coefficients and are softer than the commonly used piezoelectric ceramic materials (PZT). As a result, the total controlling voltage due to 
shunting circuits can be reduced, which is helpful when building real circuits. While it has been proved that the piezoelectric material selection would not affect the final performance of the adaptive metamaterial beam. Constitutive material and geometric parameters of the metamaterial beam used for calculations are listed in Tab. 4-1. Based on Eq. (4.10), the complex band structure of the metamaterial beam with short circuits is calculated and shown in Fig. 4-2(a). The normalized frequency $\Omega$ is defined by $\omega_{0} \omega_{0}$ where $\omega_{0}$ is the local resonant frequency of the proof mass when the PMN-PT stacks are shorted. For the metamaterial design with parameters given in Tab. $4-1$, the local resonant frequency $\left(\omega_{0} / 2 \pi\right)$ is analytically determined as $9.60 \mathrm{kHz}$. In Fig. 4-2(a), dispersion relations of evanescent flexural wave modes are manually removed, and attention is paid to the propagating flexural waves and their attenuations. According to Eqs. (4.9) and (4.10), the real part of the wavenumber represents the propagation constant of the flexural wave while the imaginary part of the wavenumber indicates the wave attenuation constant. As illustrated in Fig. 4-2(a), a wave attenuation region with $\operatorname{Im}\left(k_{x} L_{b}\right)>0$ from $\Omega=1.0$ to 1.38 (larger shaded area) is produced due to the local resonance of attached masses. It can also be observed from the figure that the real part of the wavenumber in the wave attenuation region does not equal zero, which means that the flexural wave in those frequency components is neither a purely propagating wave nor a purely evanescent wave, which will decay as it propagates along the metamaterial beam. Furthermore, due to the periodic nature of the metamaterial beam, another band gap caused by the Bragg scattering is generated and is found for $\Omega$ between 7.82 and 7.98 (smaller shaded area). However, it can be observed from the left hand side of Fig. 4-2(a) that the attenuation ability of the Bragg gap (BG) is much lower compared with that of the resonant gap (RG). 
In order to design appropriate hybrid shunting circuits to achieve extremely broadband wave attenuation, the wave band gap behavior of the metamaterial beam with various mechanical connectivities is characterized in Fig. 4-2(b). In the calculation, the mechanical connectivity $E_{\text {eff }}^{*}$ is assumed to be frequency independent in contrast with the effective modulus of the shunted piezoelectric stack. In the figure, the color legend represents the imaginary part of the normalized wavenumber. It can be observed from the figure that a passing band usually exists between the RG and BG, and the width of this passing band is smallest when the normalized modulus, $E_{\text {eff }}^{*} / E_{p}^{E}$, is around 40. Also shown in the figure, when $E_{\text {eff }}^{*} / E_{p}^{E}$ is smaller than 41 (dashed line), the RG is present at lower frequencies than that of the BG. However, if $E_{e f f}^{*} / E_{p}^{E}$ is greater than 41 , the RG will be present at higher frequencies than the BG. It is shown in the figure that if the normalized modulus, $E_{e f f}^{*} / E_{p}^{E}$, is increased from 0 to 100 , the RG can occupy very large frequency regions with $\Omega$ from 0 to 16 , while the $\mathrm{BG}$ is found at frequencies $\Omega$ from 4 to 12 . This is expected, because the resonant frequency of the proof masses is gradually increased when the modulus of the mechanical connectivity increases. In addition, it is noted that the frequency region of the RG is very narrow at lower frequencies when the mechanical connectivity has a smaller modulus, which indicates that it is impossible to achieve broadband low frequency wave attenuation with frequency independent modulus control. Therefore, proper frequency dependent modulus control algorithms will be employed to achieve broadband wave attenuation in extremely low and/or high frequency regions. 


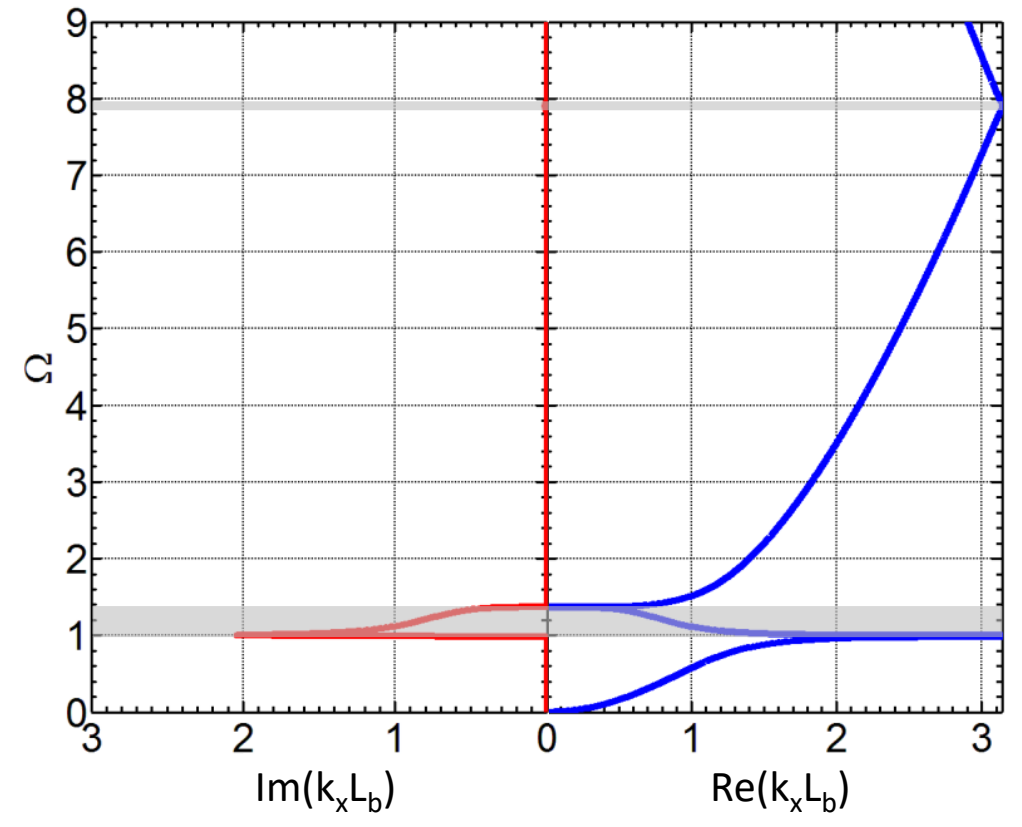

(a)

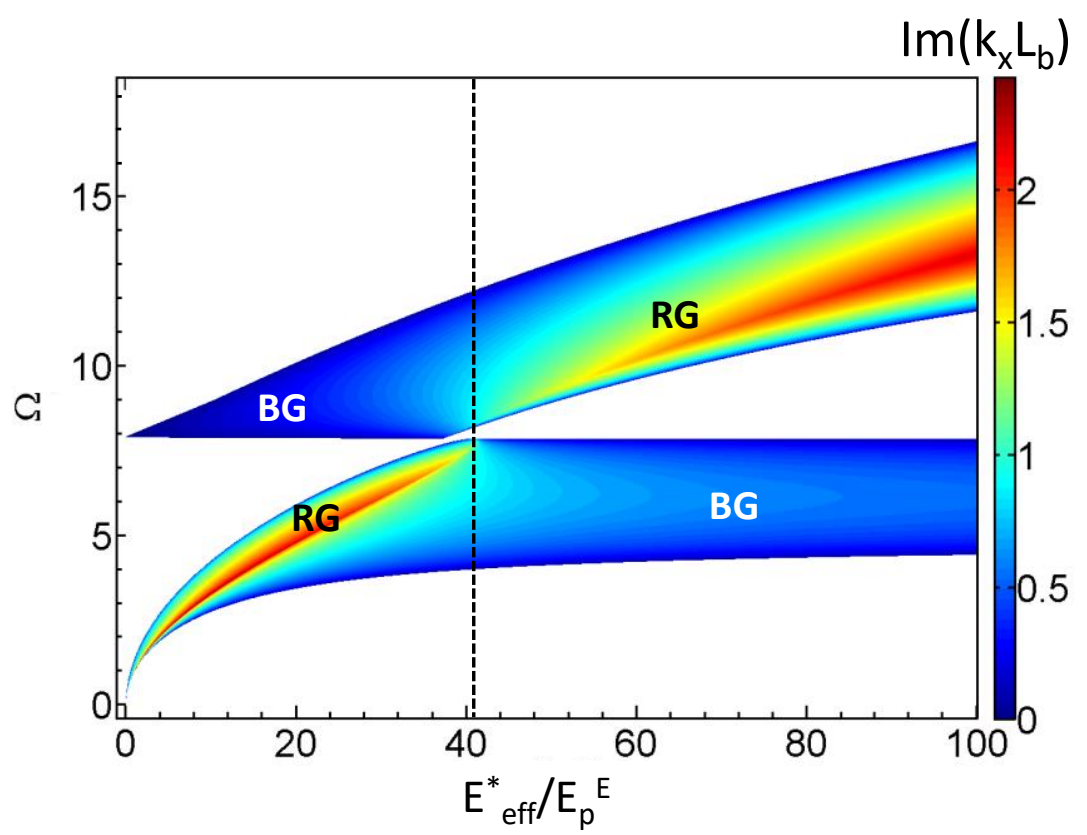

(b)

Figure 4-2 (a) Complex band structure of the adaptive metamaterial beam with short circuits; (b) Attenuation constant of flexural waves with different frequency independent normalized modulus of the mechanical connectivity 
Table 4-1 Geometric and material parameters of the constitutive materials of the proposed adaptive material beam

\begin{tabular}{|c|c|c|c|}
\hline \multicolumn{4}{|c|}{ Geometric parameters (mm) } \\
\hline$\overline{L_{b}}$ & 7.5 & $\overline{h_{m}}$ & 5.0 \\
\hline$h_{b}$ & 2.0 & $h_{p}$ & 3.0 \\
\hline$d_{m}$ & 5.0 & $a_{p}$ & 1.0 \\
\hline \multicolumn{4}{|c|}{ Material properties (Steel) } \\
\hline$\overline{E_{b}}$ & $210.0 \mathrm{GPa}$ & $\overline{G_{b}}$ & $80.8 \mathrm{GPa}$ \\
\hline$\rho_{b}$ & $7800.0 \mathrm{~kg} / \mathrm{m}_{3}$ & & \\
\hline \multicolumn{4}{|c|}{ Material properties (PMN-33\%PT) [44] } \\
\hline$\overline{c_{11}^{E}}$ & $115.0 \mathrm{GPa}$ & $\overline{e_{15}}$ & $10.1 \mathrm{C} / \mathrm{m}^{2}$ \\
\hline$c_{12}^{E}$ & $103.0 \mathrm{GPa}$ & $e_{31}$ & $-3.9 \mathrm{C} / \mathrm{m}^{2}$ \\
\hline$c_{13}{ }^{E}$ & $102.0 \mathrm{GPa}$ & $e_{33}$ & $20.3 \mathrm{C} / \mathrm{m}^{2}$ \\
\hline$c_{33}{ }^{E}$ & $103.0 \mathrm{GPa}$ & $d_{33}$ & $2820.0 \times 10^{-12} \mathrm{C} / \mathrm{N}$ \\
\hline$c_{44^{E}}$ & $69.0 \mathrm{GPa}$ & $\varepsilon_{11}^{S}$ & $1434.0 \varepsilon_{0}$ \\
\hline$c_{66}^{E}$ & $66.0 \mathrm{GPa}$ & $\varepsilon_{33}^{S}$ & $680.0 \varepsilon_{0}$ \\
\hline$S_{33^{E}}$ & $119.6 \times 10^{-12} \mathrm{~m}^{2} / \mathrm{N}$ & $\varepsilon_{33}^{T}$ & $8200.0 \varepsilon_{0}$ \\
\hline
\end{tabular}

\subsubsection{Effective modulus control of the piezoelectric stack by shunting hybrid circuits}

According to Eqs. (4.2) and (4.3), the effective modulus of the piezoelectric stack with the different hybrid circuit is calculated in function of different negative capacitances and shown in Figs 4-3(a) and (b) for TYPE I and TYPE II circuits, respectively. In the two figures, the negative inductances are selected to be the same with both $\omega_{s 1}$ and $\omega_{s 2}$ being $2.1 \pi \times 10^{4} 1 / \mathrm{s}$. As illustrated in Fig 4-3(a), for the hybrid shunting circuit connected in series (TYPE I circuit), generally at lower frequencies $(f<4 \mathrm{kHz})$, the normalized effective 
modulus, $E_{p}^{e f f} / E_{p}^{E}$, is close to the case where the stack is only shunted with the corresponding negative capacitance. This is due to the impedance of the negative inductance being very small at lower frequencies. However, at higher frequencies $(f>80$ $\mathrm{kHz}$ ), the effective modulus is near to the open circuit case, because the impedance of the negative inductance is very large at higher frequencies. When a small negative capacitance is used (i.e. $\lambda_{1}=-0.05$ ), the normalized effective modulus, $E_{p}^{\text {eff }} / E_{p}^{E}$, shows a resonant behavior (blue solid curve). It should also be noticed that this resonant behavior is different from the resonant behavior with a conventional inductance shunting circuit, where the effective modulus is decreased from the short circuit modulus to a negative value and then jump to positive infinity and gradually decreases to the open circuit modulus. For this case, the resonance behaves in an opposite way, where the modulus is always increasing instead of decreasing, due to the anti-resonant circuits. Also shown in the figure, when $\lambda_{1}$ is selected to be -1.0 and -5.0 (green dashed and red dotted curves), the resonance of the normalized effective modulus, $E_{p}^{\text {eff }} / E_{p}^{E}$, will disappear, and the modulus will be monotonously increased to the open circuit modulus. Specifically, as shown in the inset zoomed in figure of figure $3(\mathrm{a})$, it is interesting to note that the normalized effective modulus, $E_{p}^{e f f} / E_{p}^{E}$, can be gradually increased from zero at $0 \mathrm{~Hz}$ for the case with $\lambda_{1}$ being -1.0. The shape of this curve is similar to the shape of wave attenuation region at extremely low frequencies shown in Fig. 4-2(b), which indicates a great potential for broadband and extremely low frequency wave attenuation by shunting these circuits into piezoelectric stacks. 
Figure 4-3(b) shows the normalized effective modulus, $E_{p}^{\text {eff }} / E_{p}^{E}$, controlled by the hybrid shunting circuit connected in parallel (TYPE II circuit). We can see from the figure that the normalized effective modulus is close to the short circuit modulus at lower frequencies $(f<4 \mathrm{kHz})$. While, for higher frequencies, the normalized effective modulus is close to the case where the stack is only shunted with the corresponding negative capacitance. The resonance behavior of the normalized effective modulus only exists for the circuit with a relatively large negative capacitance $\left(\lambda_{2}=-0.1\right)$. Special attention will be paid to the case when $\lambda_{2}=-\left(1-k_{33}{ }^{2}\right)$, where the normalized effective modulus is increased from the short circuit modulus to positive infinity when the frequency is increased from 0 $\mathrm{Hz}$ to infinity. Because of this feature, this particular circuit has a great potential for broadband and extremely high frequency wave attenuation.

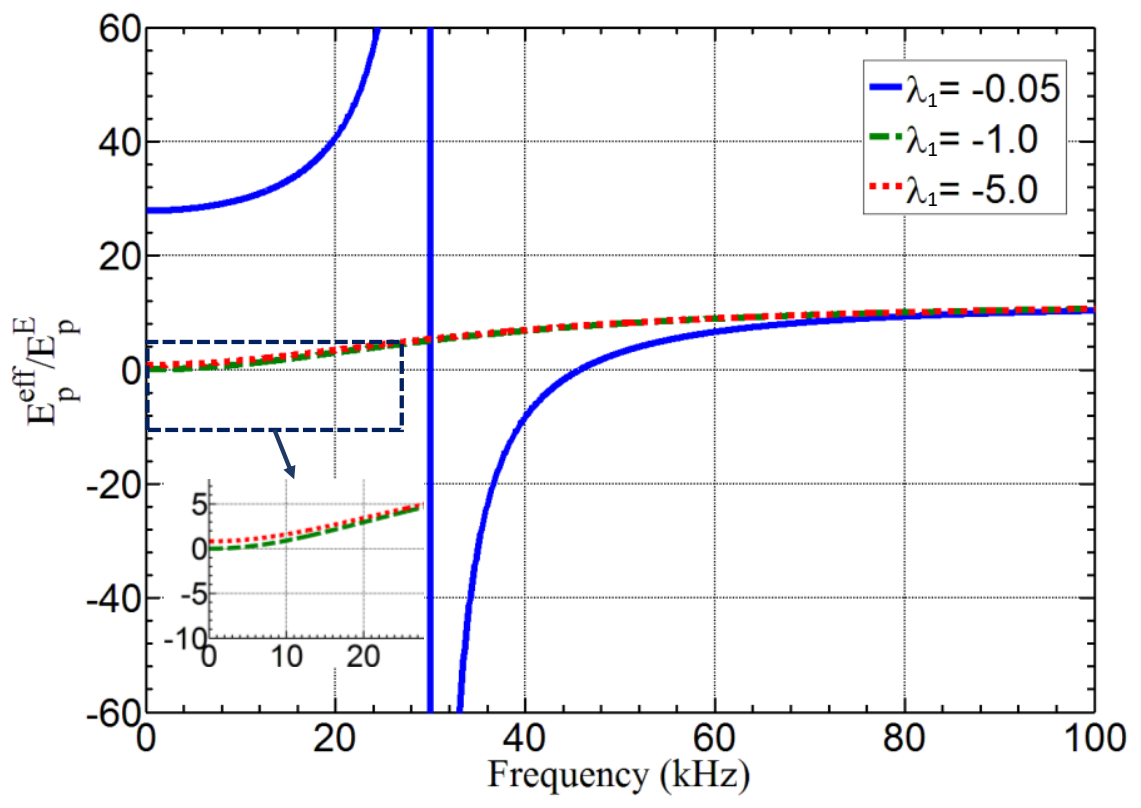

(a) 


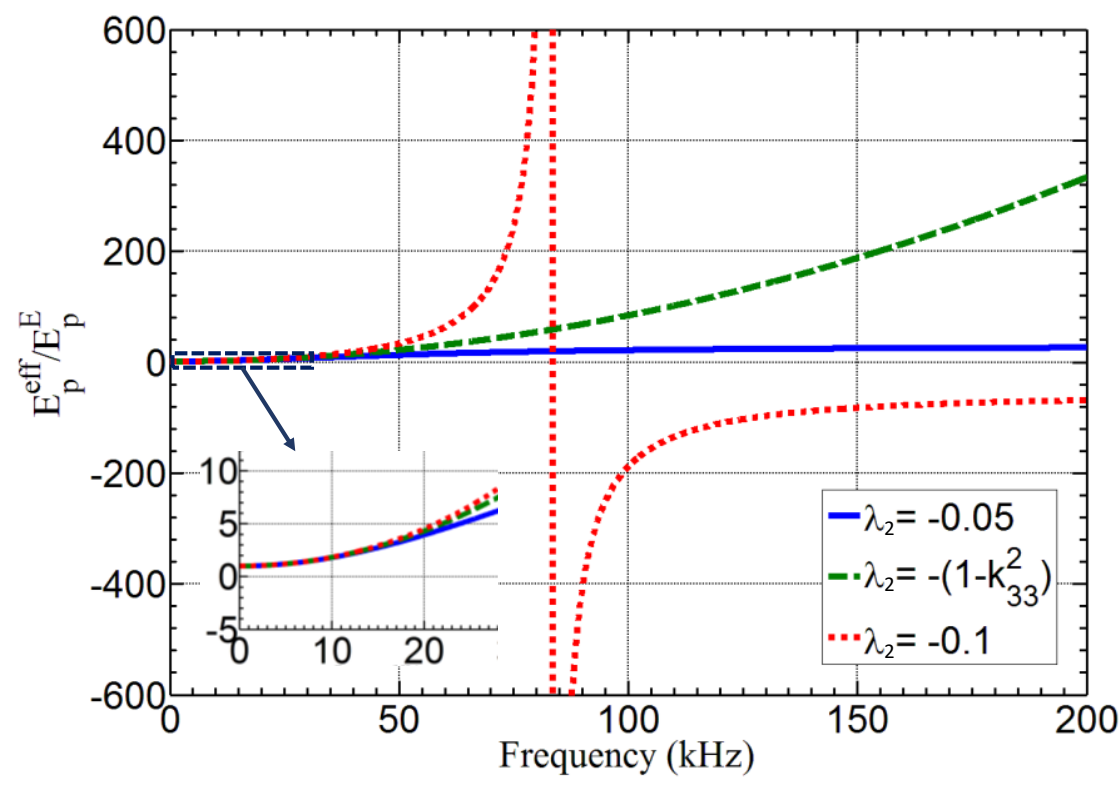

(b)

Figure 4-3 Normalized effective modulus of the piezoelectric stack shunted with two types of hybrid circuits: (a) TYPE I circuit; (b) TYPE II circuit

\subsubsection{Band gap control by hybrid shunting circuits}

Based on the frequency dependent modulus tuning of the piezoelectric stack created with the two types of hybrid shunting circuits shown in Figs. 4-3(a) and (b), the band gap control of the adaptive metamaterial beam at extremely low or high broadband frequencies is explored. To quantitatively identify band gap frequencies of the adaptive metamaterial beam, Figure 4-4(a) shows the frequency-dependent normalized effective modulus of the piezoelectric stack, $E_{p}^{e f f} / E_{p}^{E}$, for $\lambda_{1}$ being -1.0 and the normalized band gap frequency region of the metamaterial beam with the change of the mechanical connectivity, $E_{e f f}^{*} / E_{p}^{E}$, which are also plotted in Figs. 4-3(a) and 4-2(b), respectively. As shown in Fig. 4-4(a), when the frequency of the normalized effective modulus of the piezoelectric stack, 
$E_{p}^{e f f} / E_{p}^{E}$, is located within the bandgap frequency region of the passive metamaterial beam, the band gap behavior of the adaptive metamaterial beam is activated, otherwise the band gap behavior of the adaptive metamaterial beam is deactivated. By following this criteria, it is easily concluded that the band gap frequency of the adaptive metamaterial beam is only located at extremely low broadband frequencies $(\Omega=0 \sim 3.0$ (shaded area)) and the adaptive metamaterial beam will function as a conventional high-pass filter. When the normalized frequency $\Omega$ is greater than 3.0, no bandgap behavior will be observed in the adaptive metamaterial beam because the frequency of the effective modulus of the piezoelectric stack is out of the band gap frequency region of the passive metamaterial beam. As also illustrated in Fig. 4-4(a), this large wave attenuation region is caused purely by the RG. In order to validate this broadband wave attenuation, Figure 4-4(b) shows the complex wave dispersion relations of the adaptive metamaterial beam shunted with the proposed TYPE I circuits. In the figure, material and geometric parameters are unchanged with those in Figure 4-4(a). As expected, the positive imaginary part of the normalized wavenumber (attenuation constant) occupies the lower frequency region of $\Omega$ from 0 to 3.0 (shaded area), which coincides exactly with the predication in Fig. 4-4(a). Furthermore, both the real and imaginary parts of the wavenumber are half-circular shapes in the wave attenuation region. It can be concluded that flexural waves with lower frequency components $(\Omega<3.0)$ can be completely attenuated. Therefore, the adaptive metamaterial beam with TYPE I circuit can be proposed as a high-pass flexural wave filter. 


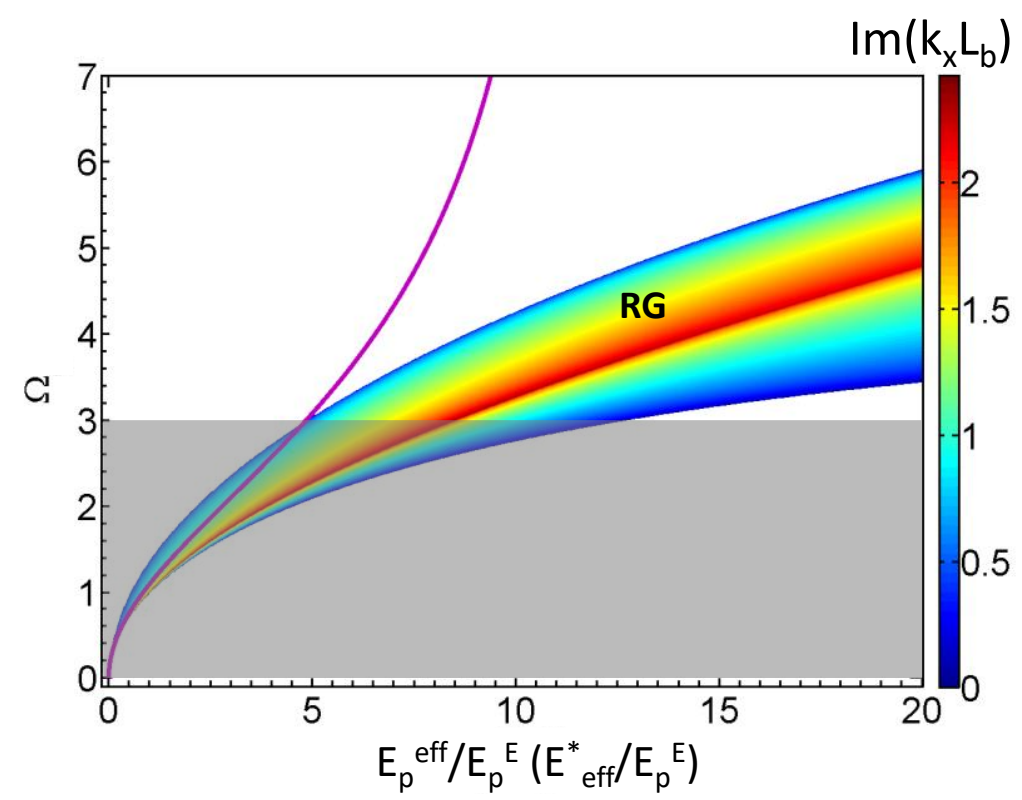

(a)

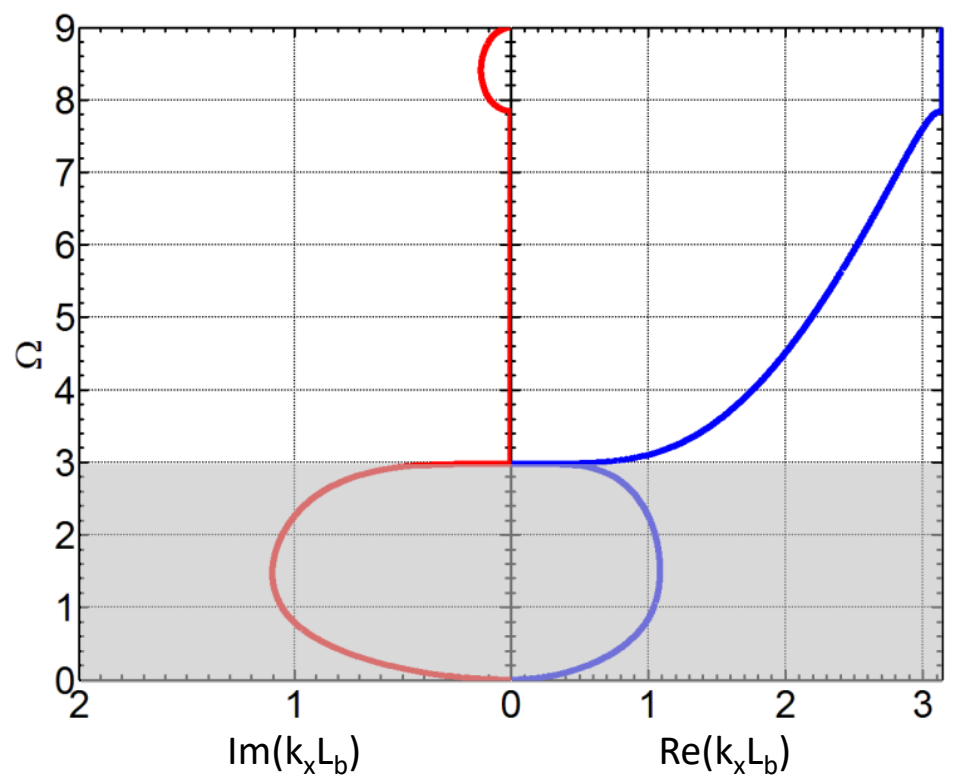

(b)

Figure 4-4 (a) Frequency dependent normalized effective modulus of the piezoelectric stack, $E_{p}^{e f f} / E_{p}^{E}$, for $\lambda_{1}$ being -1.0 and the normalized band gap frequency region of the metamaterial beam with different mechanical connectivities, $E_{e f f}^{*} / E_{p}^{E} ;$ (b) Complex band structure of the adaptive metamaterial beam with TYPE I circuits 
Figure 4-5(a) shows the frequency-dependent normalized effective modulus of the piezoelectric stack, $E_{p}^{e f f} / E_{p}^{E}$, for $\lambda_{2}$ being $-\left(1-k_{33^{2}}{ }^{2}\right)$ and the normalized band gap frequency region of the metamaterial beam with the change of the normalized mechanical connectivity, $E_{e f f}^{*} / E_{p}^{E}$, which are also plotted in Figs. 4-3(b) and 4-2(b), respectively. As shown in the figure, the effective modulus of the piezoelectric stack is located within the band gap frequency region of the passive metamaterial beam when $\Omega$ is between 1.8 and 7.8 (shaded area), which indicates an extremely large wave attenuation region at relatively high frequencies. Therefore, the adaptive metamaterial beam can function as a low-pass filter at a subwavelength scale. Similar to Fig 4-4(b), Figure 4-5(b) shows the complex wave dispersion relations of the adaptive metamaterial beam with TYPE II shunting circuits by using the same material and geometric parameters as those used in Fig. 4-5(a). As shown in the figure, the band gap frequencies are exactly identical with those predicted in Fig. 4-5(a). Furthermore, the attenuation constant (imaginary part of the wave number) is relatively uniform in the band gap frequency region (shaded area) and the attenuation ability is found to be stronger than that for lower frequency waves shown in Fig 4-4(b). In addition, the real part of the wavenumber can be used to illustrate the working mechanisms at different frequencies. For instance, at the beginning and end of the large band gap ( $\Omega=$ $1.8 \sim 2.2$ and $\Omega=6.4 \sim 7.8$ ), the normalized real part of the wavenumber is equal to $\pi$, which indicates Bragg scattering at these frequencies. At frequencies between these two regions, the wave attenuation is mainly caused by the out-of-phase motions of tunable local resonators. By changing the value of the shunted negative inductance, the working mechanism in the higher wave attenuation frequency region can be altered. 


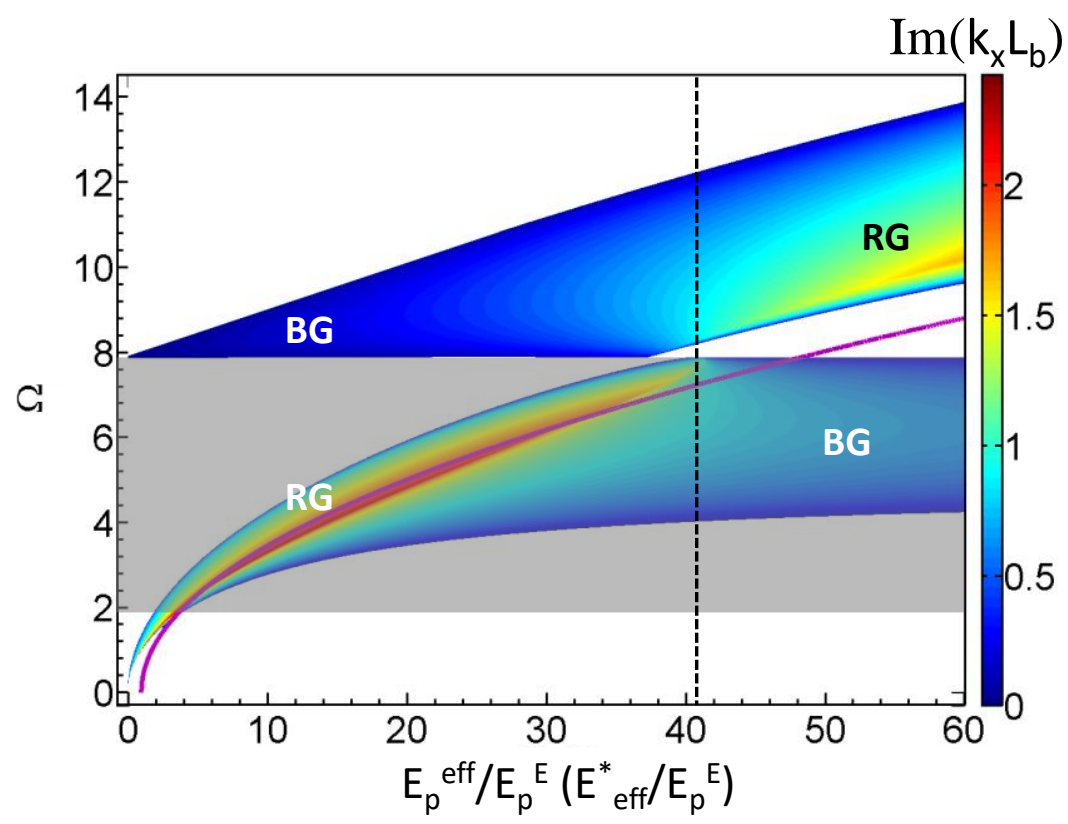

(a)

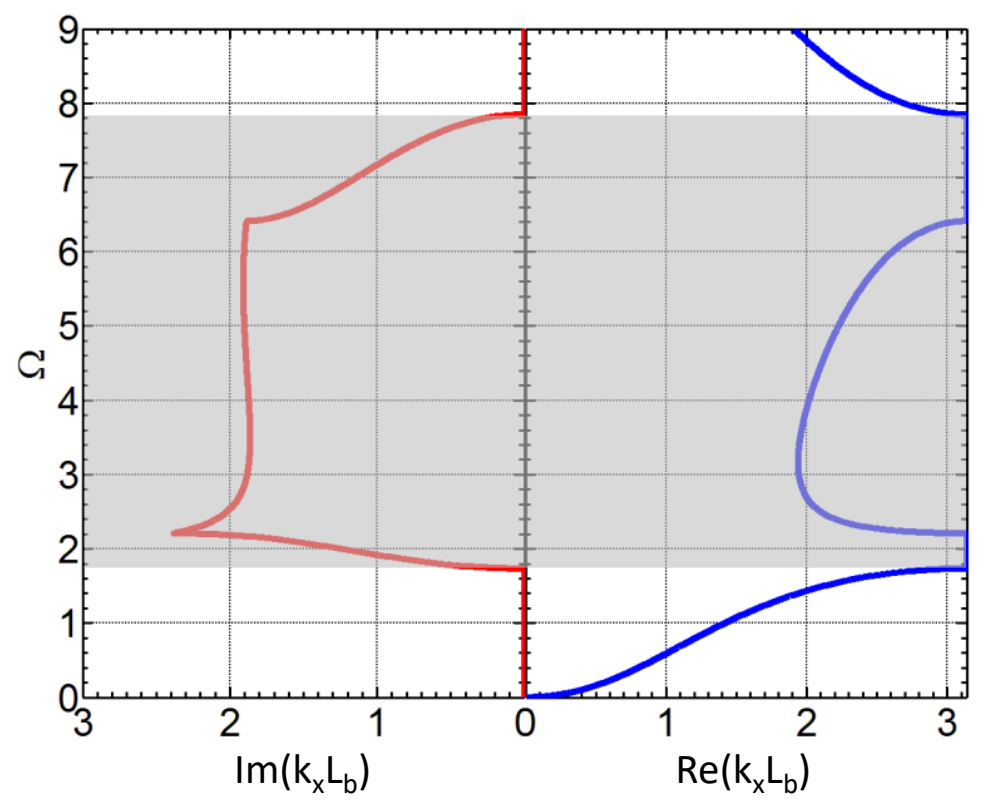

(b)

Figure 4-5 (a) Frequency dependent normalized effective modulus of the piezoelectric stack, $E_{p}^{e f f} / E_{p}^{E}$, for $\lambda_{2}$ being $-\left(1-k_{33}^{2}\right)$ and the normalized band gap frequency region of the metamaterial beam with different mechanical connectivities, $E_{e f f}^{*} / E_{p}^{E} ;$ (b) Complex band structure of the adaptive metamaterial beam with TYPE II circuits 


\subsubsection{Numerical demonstrations of wave attenuation in the adaptive metamaterial beam}

In order to examine the performance of the adaptive metamaterial beam with hybrid shunting circuits for extremely broadband low and high wave frequency attenuation, numerical simulations of wave attenuation with ten adaptive metamaterial unit cells are conducted. The schematic of these studies are shown in Fig. 4-6. In the figure, a time harmonic out of plane displacement filed, $w_{i n} e^{i \omega t}$, is prescribed on the left hand edge of the metamaterial beam. The wave attenuation properties are examined by measuring the averaged out of plane displacement amplitude ratio between the right (denoted as sensor) and left (denoted as incident) hand edges of the metamaterial beam. A 3D linear piezoelectric theory is applied to the model to describe dynamic behaviors of the shunted piezoelectric stack by using the commercial FE software, COMSOL Multiphysics.

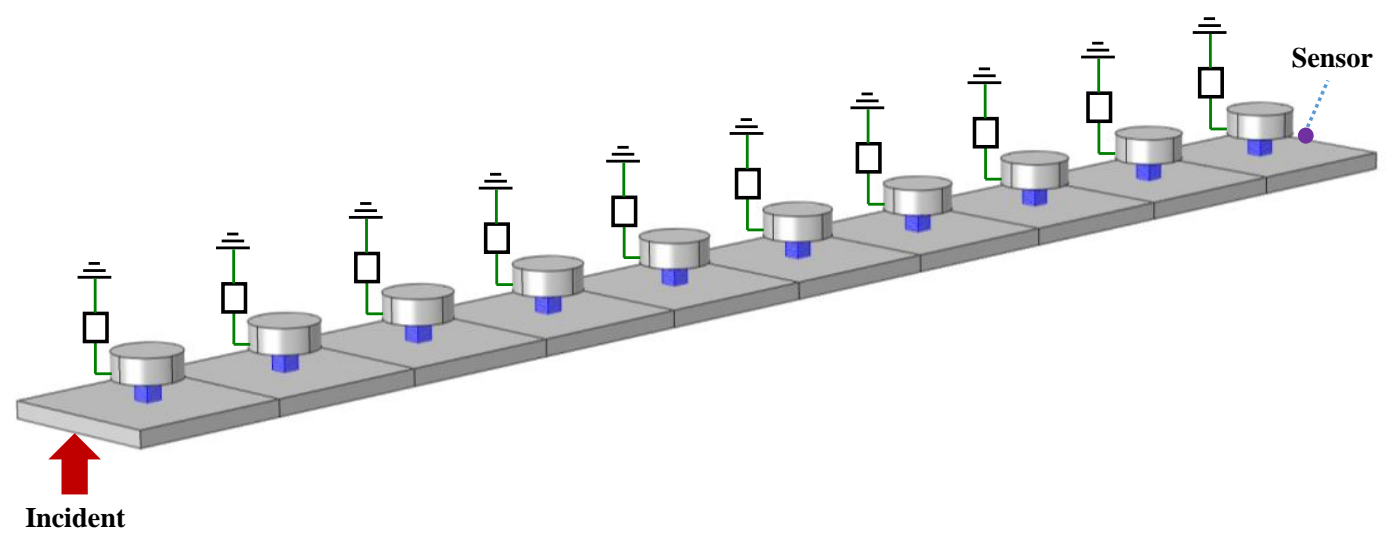

Figure 4-6 Schematic of numerical simulations of the adaptive metamaterial beam for vibration attenuation

Figure 4-7(a) shows the frequency response function of the adaptive metamaterial beam when the piezoelectric stacks are connect with short circuits. In the study, geometric and material parameters are the same as those used in Fig 4-2. Other 3D piezoelectric material 
parameters of the PMN-33\%PT is given in Tab. 4-1. As illustrated in the figure, a single narrow transmission dip is observed at frequencies between $9.4 \mathrm{kHz}$ and $13.6 \mathrm{kHz}$ (shaded area), due to out-of-phase resonant motions of proof masses. This wave attenuation region is in good agreement with the band gap frequencies predicted by the analytical dispersion relations shown in Fig. 4-2(a). Figures 4-7(b) and (c) show the frequency response functions of the adaptive metamaterial beam shunted with TYPE I and TYPE II circuit designs, respectively. In our 3D simulations, all of the boundary effects are considered. For example, the top and bottom surfaces of the piezoelectric stacks are assumed to be perfectly bonded to the host beam and proof masses, which will induce in-plane constraints on the PMN-PT stacks. Therefore, the transverse and longitudinal modes of the piezoelectric material will be coupled with each another. In order to achieve the desired performance demonstrated in Figs. 4-4(b) and 4-5(b), the shunted negative capacitance needs to be modified slightly. Specifically, for the TYPE I circuit, $\lambda_{1}$ is selected as -0.971 . As shown in Fig. 4-7(b), the frequency response function of the adaptive metamaterial beam with TYPE I circuits demonstrates the desired broadband low frequency vibration attenuation from 0 to $23.0 \mathrm{kHz}$ (shaded area), which agrees well with theoretical predictions shown in Fig. 4-4(b). To demonstrate the wave attenuation mechanism, the mode shapes of the adaptive metamaterial beam at 5 and $15 \mathrm{kHz}$ are shown in Figs. 4-8(a) and (b), respectively. It can be found that the flexural wave can be efficiently attenuated by the out-of-phase motions in the designed resonators, in which the dominated motion along $z$ direction is displayed. Furthermore, for the metamaterial beam with TYPE II circuits, $\lambda_{2}$ is selected as -0.1535 in the simulations. As expected, an extremely broadband high frequency vibration attenuation region with frequencies from 13.5 to $73.0 \mathrm{kHz}$ is observed in Fig. 4-7(c) 
(shaded area), which is almost identical with that shown in Fig. 4-5(b) found using the theoretical approach. In addition, the mode shapes of the adaptive metamaterial beam with TYPE II circuits at 30 and $60 \mathrm{kHz}$ are shown in Figs. 4-9(a) and (b), respectively. Similar as those illustrated in figure 8 , efficient wave attenuation at higher frequencies with TYPE II circuits can be observed in Fig. 4-9. It should be noted that a transmission peak exists inside the broadband transmission dip, which is caused by the rotational motions of proof masses. In the future, geometric optimizations are still needed in order to reduce or eliminate the rotational mode and obtain greater attenuation performance.

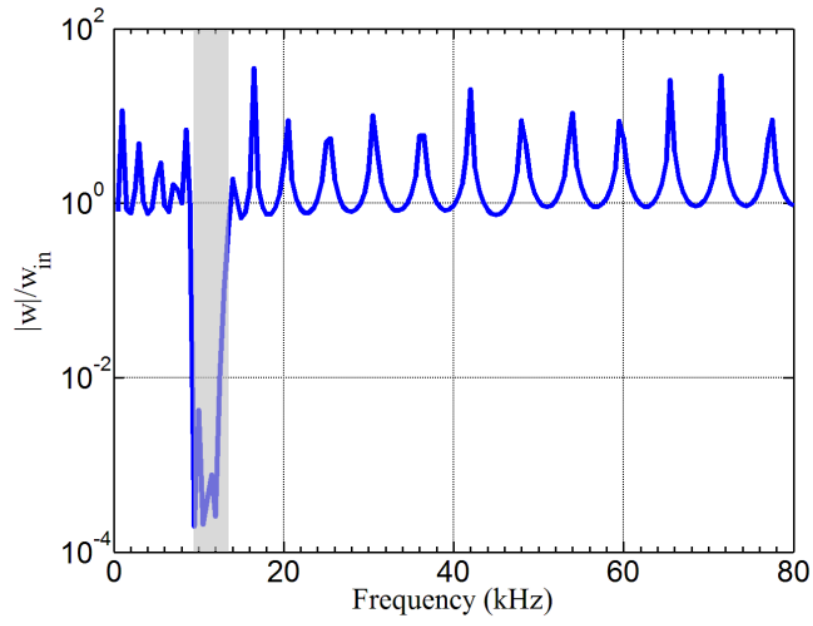

(a)

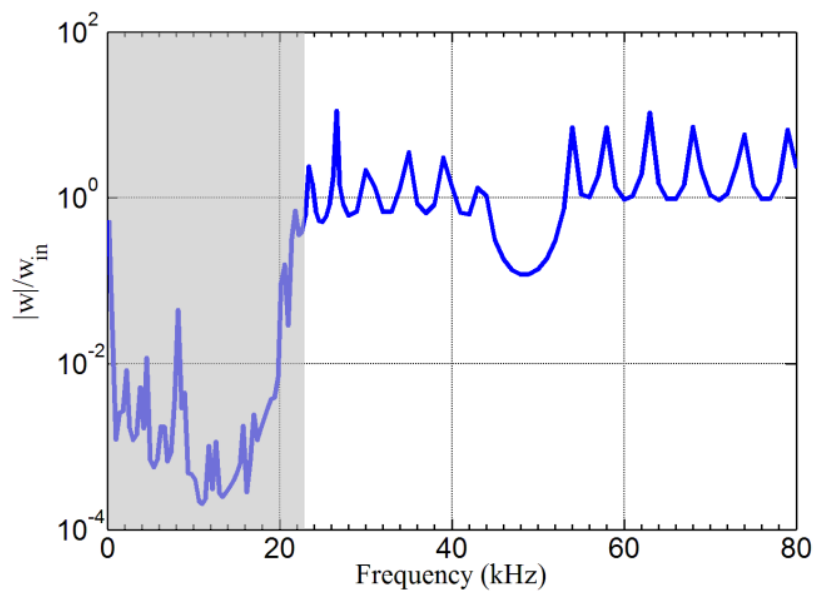

(b) 


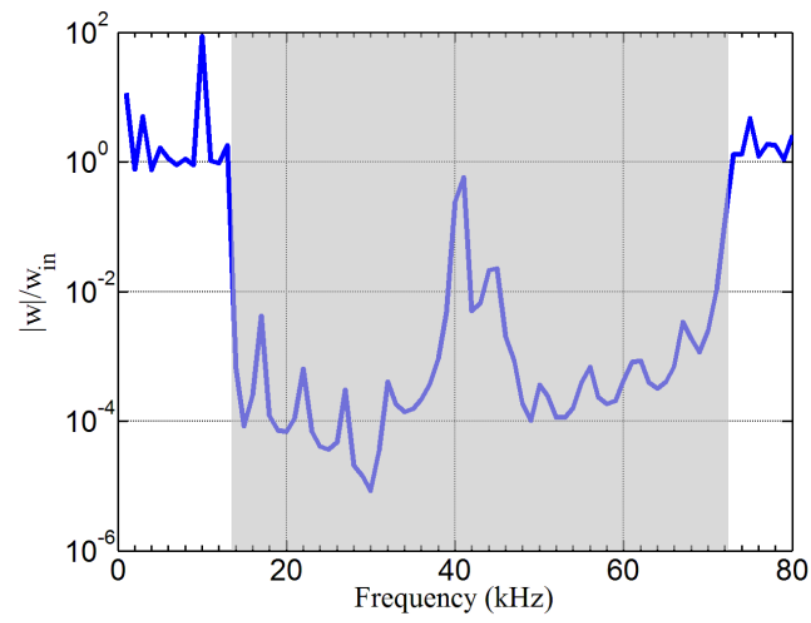

(c)

Figure 4-7 Frequency response function of the adaptive metamaterial beam: (a) short circuits; (b) TYPE I circuit; (c) TYPE II circuit

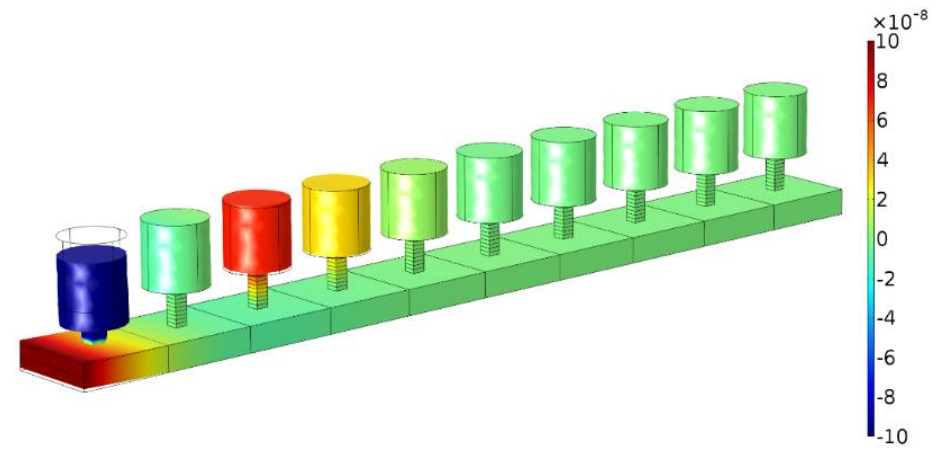

(a)

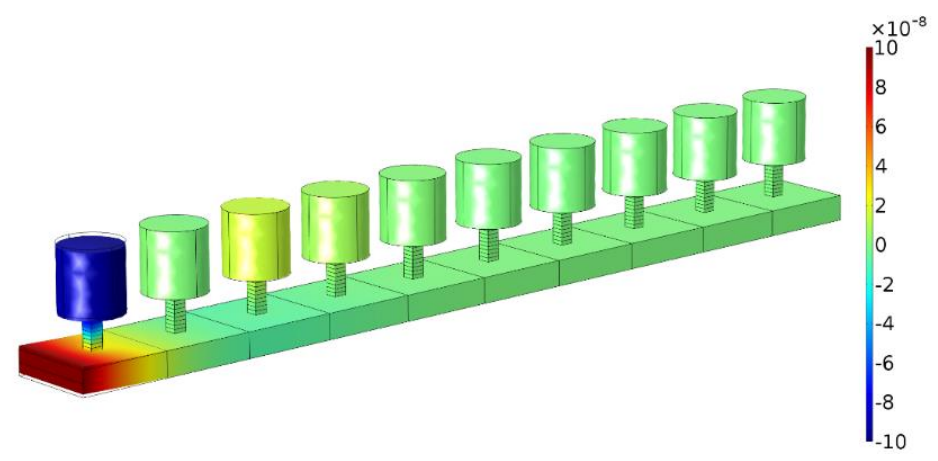

(b)

Figure 4-8 Mode shapes of a finite adaptive metamaterial beam with TYPE I circuits at different frequencies: (a) $5 \mathrm{kHz}$; (b) $15 \mathrm{kHz}$ 


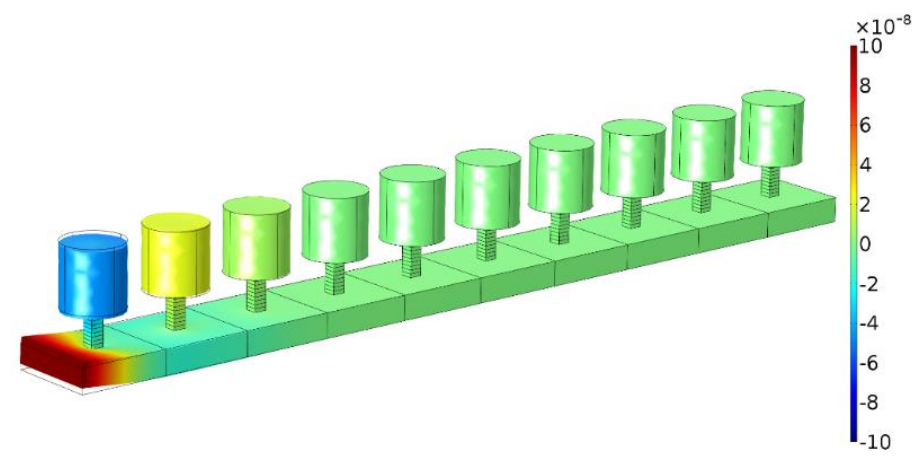

(a)

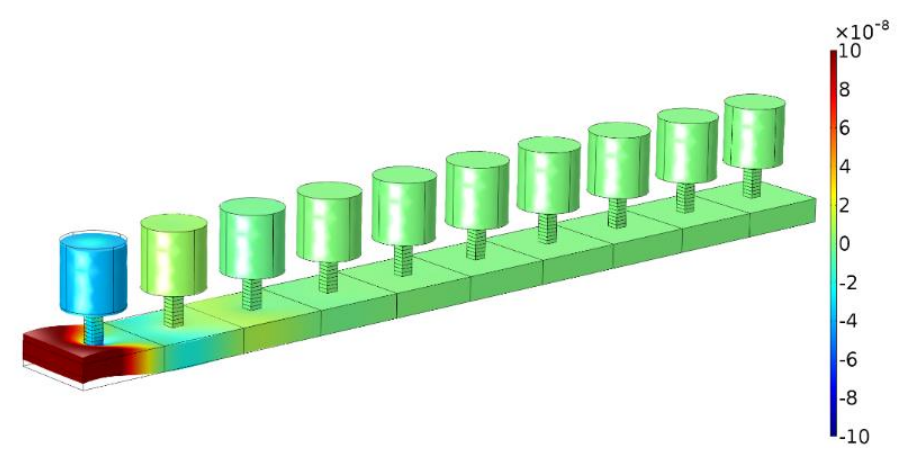

(b)

Figure 4-9 Mode shapes of a finite adaptive metamaterial beam with TYPE II circuits at different frequencies: (a) $30 \mathrm{kHz}$; (b) $60 \mathrm{kHz}$

\subsection{Conclusions}

In this chapter, we present a novel approach to achieve extremely broadband flexural wave/vibration attenuation based on tunable local resonators made of piezoelectric stacks shunted by hybrid negative capacitance and negative inductance circuits. First, wave dispersion relations of the adaptive metamaterial beam are calculated analytically by using the TM method. The unique modulus tuning properties induced by the hybrid shunting circuits are then characterized conceptually, from which the frequency dependent modulus tuning curves of the piezoelectric stack located within wave attenuation frequency regions 
are quantitatively identified. As an example, a flexural wave high-pass band filter with a wave attenuation region from 0 to $23.0 \mathrm{kHz}$ is demonstrated analytically and numerically by using the TYPE I circuit, in which the two electric components are connected in series. By changing the connection pattern to be parallel (TYPE II circuit), another super wide wave attenuation region from 13.5 to $73.0 \mathrm{kHz}$ is demonstrated to function as a low-pass filter at a subwavelength scale. In general, the proposed adaptive metamaterial design is different from the previous active metamaterial design with frequency independent modulus tuning. The proposed adaptive metamaterial possesses a super wide band gap created both naturally and artificially. Therefore, it can be used for the transient wave mitigation at extremely broadband frequencies such as blast or impact loadings. In addition, there are no theoretical power consumption within the shunting networks, because all the equivalent elements used in the circuits are capacitive and inductive. The energy exchange between the mechanical system and the electrical system do exist. When real circuits are built, the power consumption can be caused by the constitutive operational amplifiers, resistors and/or digital signal processors.

It should be noted that this chapter only discussed two specific kinds of hybrid circuits and their applications for 1D wave attenuation, although numerous other circuit combinations and wave phenomena are still unexplored. The placement of the adaptive resonators into metamaterial beams or plates can significantly enlarge their applications in flexural wave manipulations, which include but not limited in phase manipulation, superabsorption, supersensing, and one-way propagation. Those functionalities can be implemented through space and/or time variations of shunting circuits to achieve space and/or time varying mass densities. Alternatively, one can also make some geometric 
modifications on the adaptive metamaterial beam or plate to achieve and/or enhance the wave phenomenon, i.e. the adaptive resonators can be mounted on both sides of the host beam or plate symmetrically or asymmetrically; the space index gradient can be visualized through size variation of resonators. We envision that the proposed design and approach can open many possibilities in broadband vibration and wave control. 


\section{Chapter 5 A Hybrid Elastic Metamaterial with Negative Mass}

\section{Density and Tunable Bending Stiffness}

\subsection{Introduction}

In Chapters 2-4, the active/adaptive metamaterial design is focused primarily on the frequency tunability of the negative mass density by introducing variable stiffness components into the resonators. In this chapter, we will propose a new class of adaptive hybrid metamaterials by integrating negative capacitance shunted piezoelectric patches into a passive locally resonant metamaterial, in which the negative effective mass density mechanism produced by the passive metamaterial and extremely tunable effective bending stiffness from the piezoelectric components are used in parallel. By incorporating both wave control mechanisms simultaneously, the active phononic crystal can be used to actively "enhance" or "eliminate" (switch ON/OFF) the wave attenuation properties while also allowing for the control of the double negative material properties over an extremely broad frequency range. To demonstrate the broadband wave manipulation ability, a comprehensive multi-physical analytical model is developed by considering locally resonant motions, electro-mechanical coupling and shunting circuit effects. Specifically, the switchable bandgap and the broadest negative refraction region controlled by electric circuits are quantitatively characterized and interpreted by dynamic effective parameters (effective mass density and bending stiffness) to illustrate the underlying wave control mechanism. Finally, three-dimensional (3D) multi-physical wave analyses on a finite hybrid metamaterial beam and plates are conducted to validate the tunable wave propagation manipluation. The proposed hybrid metamaterial could significantly impact 
research areas including tunable or switchable low-frequency wave/vibration attenuation, wave guiding, wave imaging and various other wave control applications.

\subsection{Design and multi-physical modeling of the adaptive hybrid}

\section{metamaterial beam}

This study explores the array of potential applications offered by integrating a locally resonant passive metamaterial microstructure with an adaptive phononic crystal to construct a new adaptive hybrid metamaterial and achieve adaptive wave control as illustrated in Fig. 5-1. The locally resonant passive metamaterial component is created by bonding a periodic array of rubber disks (working as springs) and metal cylinders (working as lumped masses) onto the surface of a host beam as shown in Fig. 5-1(a) for wave attenuation at subwavelength frequency region. Next, the adaptive phononic beam component is constructed with a periodic array of piezoelectric patches shunted with negative capacitance controlling circuits as shown in Fig. 5-1(b). Finally, the adaptive hybrid metamaterial beam is physically realized by combining these two microstructural designs as shown in Fig. 5-1(c). In the design, the shunted piezoelectric patches and the mechanical resonators are equally spaced with the origins of the resonators and piezoelectric patches located in the same position. The negative capacitance of the shunting circuits is denoted as $c_{N}$. The fundamental concept behind this design is the integration of the extreme bending stiffness tunability (softening, hardening, and negative stiffness) provided by the shunted piezoelectric patches with the negative mass density mechanism of the passive metamaterial. By combining both wave control mechanisms into a single system, tunable wave manipulation and control including negative refraction over a broad 
frequency range can be achieved in real-time and without physical modification of the microstructures.

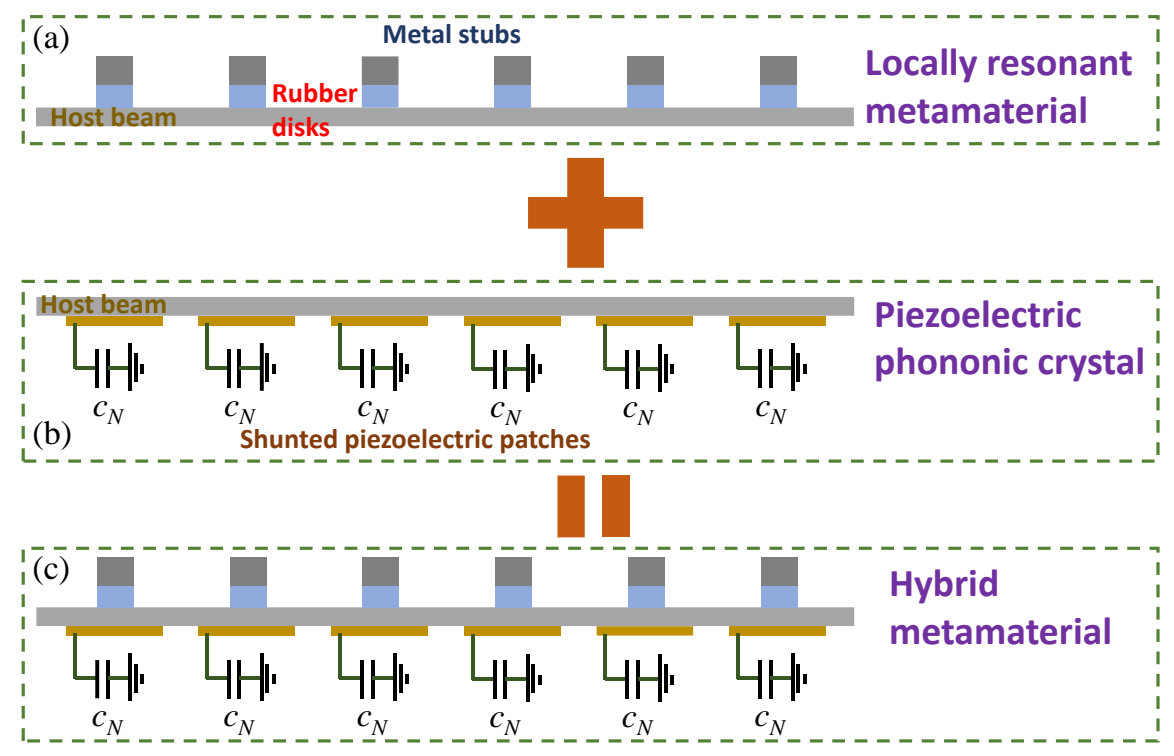

Figure 5-1 (a) Schematic of the passive locally resonant metamaterial beam. (b)

Schematic of the adaptive piezoelectric phononic beam. (c) Schematic of the proposed adaptive hybrid metamaterial beam

\subsubsection{Electro-mechanical modeling of the hybrid metamaterial beam}

To demonstrate how the hybrid metamaterial behaves for different values of negative capacitances at different frequencies and understand the intrinsic physics and working mechanisms, an analytical model is developed, which will serve as the theoretical foundation and an efficient tool during the wave analysis and microstructure design for tunable wave manipulations. For simplicity, a lumped mass model is adopted for a mechanical resonator by considering both the compressed and stretched deformation in the rubber disk (lumped spring with the stiffness being $k_{s}=\frac{E_{r} \pi\left(d_{m} / 2\right)^{2}}{h_{r}}$, where $h_{r}, d_{m}$ and 
$E_{r}$ represent the height, diameter and Young's modulus of the rubber material, and the boundary conditions on the top and bottom surfaces of the disk are ignored) as well as the kinematic motion of the metal cylinder (lumped mass) for the metamaterial unit cell illustrated in Fig. 5-2 and the lattice constant and the length of the piezoelectric patch denoted as $L_{b}$ and $L_{p}$, respectively. The thicknesses of the piezoelectric patch and the host beam are denoted as $h_{p}$ and $h_{b}$, respectively. In order to follow the procedures of the transfer matrix method, the unit cell of the adaptive hybrid metamaterial is then divided into three sections to consider continuity boundary conditions.

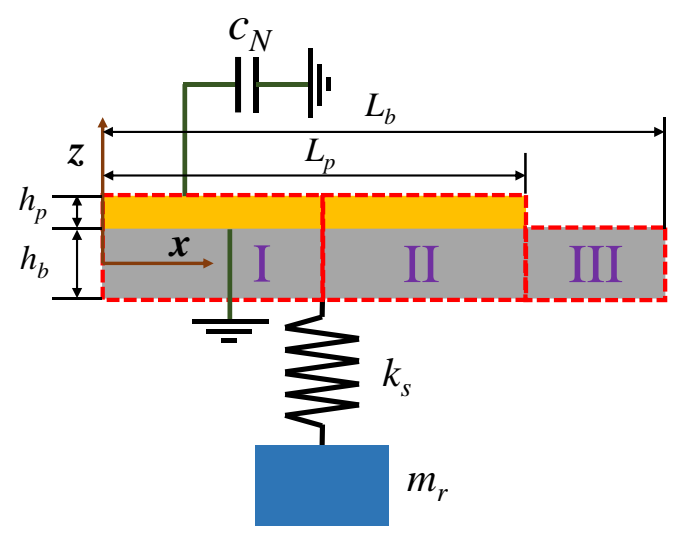

Figure 5-2 Schematic of the 2D analytical modeling of the adaptive hybrid metamaterial beam

Based on the plane-stress assumption $\left(T_{2}=T_{12}=T_{23}=0, S_{12}=S_{23}=0\right)$ and assuming $T_{3}=0$, due to the traction free boundaries on the top and bottom surfaces of the hybrid metamaterial beam, the linear constitutive relations of the piezoelectric material can be simplified and written as [48]

$$
S_{1}=s_{11}^{E} T_{1}+d_{31} E_{3}
$$

$D_{3}=d_{31} T_{1}+\varepsilon_{33}^{T} E_{3}$ 
where $S_{1}, T_{1}, D_{3}$ and $E_{3}$ represent the strain and stress along the $x$-direction and the electric displacement and electric field along the z-direction, respectively. $s_{11}{ }^{E}, \varepsilon_{33}^{T}$ and $d_{31}$ denote the compliance coefficient at constant electric field, dielectric coefficient at constant stress and piezoelectric coefficient, respectively. The relationship between the electric field and electric potential in the piezoelectric patch can be expressed as [48]

$$
E_{3}=-\frac{\partial V}{\partial z}
$$

For sections I and II of the host beam, the displacement along the $x$-direction is assumed to be linearly distributed along the $z$-direction in the form

$$
u(x, z)=u_{0}(x)-z \frac{\partial w}{\partial x}
$$

where $u_{0}$ and $w$ denote the in-plane and out-of-plane displacements at the mid-plane of the host beam $(z=0)$, respectively. It should be noted that the beam displacement fields can be written on an axis in any position of the beam. By using Eq. (5.3), it will be more convenient to consider continuous boundary conditions at the interface between the beam section with the piezoelectric patch and the beam section without the piezoelectric patch. It should be mentioned that the thickness of the piezoelectric patch is comparable to the thickness of the host beam in the current design and therefore, the assumption of a constant electric field along the thickness direction will not be valid. To consider this factor, a linearly distributed electric field along the thickness direction and the corresponding electric potential should be assumed as

$$
E_{3}(x, z)=z \cdot a(x)+b(x)
$$




$$
V(x, z)=-\left[\frac{a(x)}{2} z^{2}+b(x) z+c(x)\right]
$$

where $a, b$ and $c$ are distribution functions to be determined by the piezoelectric governing equations and corresponding electrical boundary conditions.

By substituting Eqs. (5.3) and (5.4a) into Eq. (5.1) and considering the electrical governing equation written as [48]

$$
\frac{\partial D_{3}}{\partial z}=0
$$

the distribution function, $a$, is obtained as

$$
a=\frac{d_{31}}{s_{11}^{E} \varepsilon_{33}^{T}-d_{31}^{2}} \frac{\partial^{2} w}{\partial x^{2}}
$$

As illustrated in Fig. 5-2(b), the lower electrode of the piezoelectric patch is grounded, and the upper electrode of the piezoelectric patch is connected to a shunting circuit with an impedance of $Z_{s h}=\frac{1}{i \omega c_{N}}$, which yields

$$
\begin{aligned}
& V\left(z=\frac{h_{b}}{2}\right)=0 \\
& V\left(z=\frac{h_{b}}{2}+h_{p}\right)=-i \omega Z_{s h} \cdot Q
\end{aligned}
$$

where $Q=\int_{0}^{L_{p}}-D_{3} d x$ is the free charge on the upper electrode of the piezoelectric patch. 
By combining Eqs. (5.4), (5.6), (5.7a) and (5.7b), the distribution function $b$ can be obtained as

$$
b=-\frac{A}{h_{p}}-\frac{a}{2}\left(h_{b}+h_{p}\right)
$$

where the electric potential on the upper electrode,

$$
A=\frac{i \omega \frac{d_{31} Z_{s h}}{s_{11}^{E}} \int_{0}^{L_{p}}\left(\frac{\partial u_{0}}{\partial x}-\frac{h_{b}+h_{p}}{2} \frac{\partial^{2} w}{\partial x^{2}}\right) d x}{1+i \omega C_{p}^{T}\left(1-k_{31}^{2}\right) Z_{s h}}
$$

with $k_{31}^{2}=\frac{d_{31}^{2}}{s_{11}^{E} \varepsilon_{33}^{T}}$ and $C_{p}^{T}$ is the electro-mechanical coupling coefficient of the piezoelectric material and the capacitance of the piezoelectric patch at constant stress, respectively.

According to the Euler-Bernoulli beam theory, the bending moment and normal force in the mid-plane of beam sections I and II are [48]

$$
\begin{aligned}
& M=\int_{-\frac{h_{b}}{2}}^{\frac{h_{b}}{2}}-T_{1}^{(b)} z d z+\int_{\frac{h_{b}}{2}}^{\frac{h_{b}}{2}+h_{p}}-T_{1} z d z \\
& N=\int_{-\frac{h_{b}}{2}}^{\frac{h_{b}}{2}} T_{1}^{(b)} d z+\int_{\frac{h_{b}}{2}}^{\frac{h_{b}}{2}+h_{p}} T_{1} d z
\end{aligned}
$$

where $T_{1}^{(b)}$ denotes the normal stress on the cross section of the host beam. Substituting Eq. (5.1a) into Eqs. (5.9a) and (5.9b), the coupled bending moment, normal and shear forces can be obtained as 


$$
\begin{aligned}
& M=I \frac{\partial^{2} w}{\partial x^{2}}-J \frac{\partial u_{0}}{\partial x}-F A \\
& N=K \frac{\partial u_{0}}{\partial x}-J \frac{\partial^{2} w}{\partial x^{2}}+G A \\
& T=I \frac{\partial^{3} w}{\partial x^{3}}-J \frac{\partial^{2} u_{0}}{\partial x^{2}}
\end{aligned}
$$

where

$$
\begin{aligned}
& I=\frac{E h_{b}^{3}}{12}+\frac{6 h_{b} h_{p}^{2}+3 h_{b}^{2} h_{p}+4 h_{p}^{3}}{12 s_{11}^{E}}+\frac{h_{p}^{3} d_{31}^{2}}{12 s_{11}^{E}\left(s_{11}^{E} \varepsilon_{33}^{T}-d_{31}^{2}\right)}, \\
& J=\frac{h_{p}^{2}+h_{b} h_{p}}{2 s_{11}^{E}}, \\
& K=E h_{b}+\frac{h_{p}}{s_{11}^{E}} \\
& F=\frac{\left(h_{b}+h_{p}\right) d_{31}}{2 s_{11}^{E}} \\
& G=\frac{d_{31}}{s_{11}^{E}}
\end{aligned}
$$

with $E$ being the Young's modulus of the host beam.

It should be noted that the electric shunting effects are now explicitly included in the bending moment and normal force, which will be used for the derivation of the governing equation of the metamaterial. 
Based on the force and moment balance, the electro-mechanically coupled equations of motion for flexural and longitudinal waves on the metamaterial beam sections I and II can be expressed as

$$
\begin{gathered}
I \frac{\partial^{4} w}{\partial x^{4}}-J \frac{\partial^{3} u_{0}}{\partial x^{3}}+\bar{\rho} \frac{\partial^{2} w}{\partial t^{2}}=0 \\
K \frac{\partial^{2} u_{0}}{\partial x^{2}}-J \frac{\partial^{3} w}{\partial x^{3}}-\bar{\rho} \frac{\partial^{2} u_{0}}{\partial t^{2}}=0
\end{gathered}
$$

where $\bar{\rho}=\rho_{b} h_{b}+\rho_{p} h_{p}$ with $\rho_{b}$ and $\rho_{p}$ denoting the mass densities of the host beam and the piezoelectric patch, respectively.

For section III of the beam, the governing equations for flexural and longitudinal waves on the host beam can be written as [48]

$$
\begin{aligned}
& I^{(b)} \frac{\partial^{4} w^{(b)}}{\partial x^{4}}+\rho^{(b)} \frac{\partial^{2} w^{(b)}}{\partial t^{2}}=0 \\
& K^{(b)} \frac{\partial^{2} u_{0}^{(b)}}{\partial x^{2}}-\rho^{(b)} \frac{\partial^{2} u_{0}^{(b)}}{\partial t^{2}}=0
\end{aligned}
$$

where

$$
\begin{gathered}
I^{(b)}=\frac{E h_{b}^{3}}{12}, \\
K^{(b)}=E h_{b}, \\
\rho^{(b)}=\rho_{b} h_{b},
\end{gathered}
$$


with $w^{(b)}$ and $u_{0}^{(b)}$ representing the out-of-plane and in-plane displacements on the midplane of the host beam in section III, respectively.

\subsubsection{Wave dispersive analysis}

The general harmonic wave solutions in the metamaterial beam with a piezoelectric patch can be assumed as

$$
\begin{aligned}
& w=\bar{A} e^{i \omega t+\lambda x} \\
& u_{0}=\bar{B} e^{i \omega t+\lambda x}
\end{aligned}
$$

By applying Eqs. (5.13a) and (5.13b) to Eqs. (5.11a) and (5.11b), a characteristic equation can be obtained as

$$
\left(I K-J^{2}\right) \lambda^{6}+I \omega^{2} \bar{\rho} \lambda^{4}-K \omega^{2} \bar{\rho} \lambda^{2}-\omega^{4} \bar{\rho}^{2}=0
$$

By finding the six roots of the polynomial given by Eq. (5.14), the general wave solutions can be expressed as

$$
\begin{aligned}
& w=\sum_{n=1}^{6} \bar{A}_{n} e^{\lambda_{n} x} \\
& u_{0}=\sum_{n=1}^{6} \bar{A}_{n} \alpha_{n} e^{\lambda_{n} x}
\end{aligned}
$$

where $\lambda_{n}$ are the six roots of Eq. (5.14), $\bar{A}_{n}$ are arbitrary constants, and $\alpha_{n}=\frac{I \lambda_{n}^{4}-\omega^{2} \bar{\rho}}{J \lambda_{n}^{3}}$. It should be noted that all time-harmonic terms are suppressed in the equations for brevity. 
Specifically, for sections I and II in the beam the flexural and longitudinal waves can then be expressed by

$$
\begin{aligned}
& w^{(\mathrm{I})}=\sum_{n=1}^{6} \bar{A}_{n}^{(\mathrm{I})} e^{\lambda_{n} x}, 0 \leq x \leq \frac{L_{p}}{2} \\
& u_{0}^{(\mathrm{I})}=\sum_{n=1}^{6} \bar{A}_{n}^{(\mathrm{I})} \alpha_{n} e^{\lambda_{n} x}, 0 \leq x \leq \frac{L_{p}}{2}
\end{aligned}
$$

and

$$
\begin{aligned}
& w^{(\mathrm{II})}=\sum_{n=1}^{6} \bar{A}_{n}^{(\mathrm{II})} e^{\lambda_{n} x}, \frac{L_{p}}{2} \leq x \leq L_{p} \\
& u_{0}^{(\mathrm{II})}=\sum_{n=1}^{6} \bar{A}_{n}^{(\mathrm{II})} \alpha_{n} e^{\lambda_{n} x}, \frac{L_{p}}{2} \leq x \leq L_{p}
\end{aligned}
$$

Based on the expression of the total electrical potential on the upper electrode of the shunted piezoelectric patch (Eq. (5.8b)), $A$ can be separated into two parts from the beams sections I and II as

$$
A=\sum_{n=1}^{6}\left(\bar{A}_{n}^{(\mathrm{I})} \beta_{n}^{(\mathrm{I})}+\bar{A}_{n}^{(\mathrm{II})} \beta_{n}^{(\mathrm{II})}\right)
$$

in which

$$
\begin{aligned}
& \beta_{n}^{(\mathrm{I})}=\frac{i \omega \frac{d_{31} Z_{s h}}{s_{11}^{E}}\left(\alpha_{n}-\frac{h+h_{p}}{2} \lambda_{n}\right)\left(e^{\frac{\lambda_{n} L_{p}}{2}}-1\right)}{1+i \omega C_{p}^{T}\left(1-k_{31}^{2}\right) Z_{s h}}, \\
& \beta_{n}^{(\mathrm{II})}=\frac{i \omega \frac{d_{31} Z_{s h}}{s_{11}^{E}}\left(\alpha_{n}-\frac{h+h_{p}}{2} \lambda_{n}\right)\left(e^{\lambda_{n} L_{p}}-e^{\frac{\lambda_{n} L_{p}}{2}}\right)}{1+i \omega C_{p}^{T}\left(1-k_{31}^{2}\right) Z_{s h}} .
\end{aligned}
$$


For section III of the beam, the flexural and longitudinal waves can then be expressed as

$$
\begin{aligned}
& w^{(\mathrm{III})}=\sum_{n=1}^{4} \bar{A}_{n}^{(\mathrm{III})} e^{\lambda_{n}^{(b)} x} \\
& u_{0}^{(\mathrm{III})}=\sum_{n=5}^{6} \bar{A}_{n}^{(\mathrm{III})} e^{\lambda_{n}^{(b)} x}
\end{aligned}
$$

where the six roots in the characteristic equation are readily obtained as

$\lambda_{1,2}^{(b)}= \pm i \sqrt[4]{\rho^{(b)} \omega^{2} / I^{(b)}}$

$\lambda_{3,4}^{(b)}= \pm \sqrt[4]{\rho^{(b)} \omega^{2} / I^{(b)}}$

$\lambda_{5,6}^{(b)}= \pm i \sqrt{\rho^{(b)} \omega^{2} / E}$.

The wave transfer matrix method will then be used to obtain the dispersion relations of the hybrid metamaterial beam. To implement this method, wave field quantities including the displacement, rotational angle, bending moment and force fields in the center of the shunted piezoelectric patch $\left(x=\frac{L_{p}}{2}\right)$ in the beam sections I and II within a unit cell of the periodic system (as shown in Fig. 2 (b)) are rewritten in the matrix format as

$$
\mathbf{Y}_{\mathbf{p}}^{(\mathbf{X})}\left(x=\frac{L_{p}}{2}\right)=\mathbf{B}_{\mathbf{p}}\left(x=\frac{L_{p}}{2}\right) \overline{\mathbf{A}}^{(\mathbf{x})}+\mathbf{Y}_{\mathbf{A}}, \mathbf{X}=\mathbf{I}, \mathbf{I I}
$$

where

$$
\mathbf{Y}_{\mathbf{A}}=\mathbf{F}^{(\mathbf{I})} \overline{\mathbf{A}}^{(\mathbf{I})}+\mathbf{F}^{(\mathrm{II})} \overline{\mathbf{A}}^{(\mathbf{I I})}
$$


with

$$
\begin{aligned}
& \mathbf{Y}_{\mathbf{p}}^{(\mathbf{X})}(x)=\left[w^{(\mathrm{X})}(x) \frac{\partial w^{(\mathrm{X})}(x)}{\partial x} M^{(\mathrm{X})}(x) T^{(\mathrm{X})}(x) u_{0}^{(\mathrm{X})}(x) N^{(\mathrm{X})}(x)\right]^{\mathrm{T}}, \\
& \overline{\mathbf{A}}^{(\mathrm{x})}=\left[\begin{array}{llllll}
\bar{A}_{1}^{(\mathrm{X})} & \bar{A}_{2}^{(\mathrm{X})} & \bar{A}_{3}^{(\mathrm{x})} & \bar{A}_{4}^{(\mathrm{X})} & \bar{A}_{5}^{(\mathrm{x})} & \bar{A}_{6}^{(\mathrm{x})}
\end{array}\right]^{\mathrm{T}}, \\
& \mathbf{B}_{\mathbf{p}}(x)=\left[\begin{array}{llllll}
a_{11} & a_{12} & a_{13} & a_{14} & a_{15} & a_{16} \\
a_{21} & a_{22} & a_{23} & a_{24} & a_{25} & a_{26} \\
a_{31} & a_{32} & a_{33} & a_{34} & a_{35} & a_{36} \\
a_{41} & a_{42} & a_{43} & a_{44} & a_{45} & a_{46} \\
a_{51} & a_{52} & a_{53} & a_{54} & a_{55} & a_{56} \\
a_{61} & a_{62} & a_{63} & a_{64} & a_{65} & a_{66}
\end{array}\right],
\end{aligned}
$$

with

$a_{1 n}=e^{\lambda_{n} x}$,

$a_{2 n}=\lambda_{n} e^{\lambda_{n} x}$,

$a_{3 n}=\left(I \lambda_{n}^{2}-J \alpha_{n} \lambda_{n}\right) e^{\lambda_{n} x}$,

$a_{4 n}=\left(I \lambda_{n}^{3}-J \alpha_{n} \lambda_{n}^{2}\right) e^{\lambda_{n} x}$,

$a_{5 n}=\alpha_{n} e^{\lambda_{n} x}$,

$a_{6 n}=\left(K \alpha_{n} \lambda_{n}-J \lambda_{n}^{2}\right) e^{\lambda_{n} x}$,

and 


$$
\mathbf{F}^{(\mathrm{x})}=\left[\begin{array}{cccccc}
0 & 0 & 0 & 0 & 0 & 0 \\
0 & 0 & 0 & 0 & 0 & 0 \\
-F \beta_{1}^{(\mathrm{X})} & -F \beta_{2}^{(\mathrm{X})} & -F \beta_{3}^{(\mathrm{X})} & -F \beta_{4}^{(\mathrm{x})} & -F \beta_{5}^{(\mathrm{x})} & -F \beta_{6}^{(\mathrm{X})} \\
0 & 0 & 0 & 0 & 0 & 0 \\
0 & 0 & 0 & 0 & 0 & 0 \\
G \beta_{1}^{(\mathrm{X})} & G \beta_{2}^{(\mathrm{X})} & G \beta_{3}^{(\mathrm{X})} & G \beta_{4}^{(\mathrm{X})} & G \beta_{5}^{(\mathrm{X})} & G \beta_{6}^{(\mathrm{X})}
\end{array}\right] .
$$

For simplification, the vertical inertial force will be used in the central area of the piezoelectric patch to represent the mechanical resonator. Therefore, the continuity boundary conditions at the interface between beam sections I and II must satisfy the condition

$$
\mathbf{Y}_{\mathbf{p}}^{(\mathrm{I})}\left(x=\frac{L_{p}}{2}\right)=\mathbf{Y}_{\mathbf{p}}^{(\mathrm{II})}\left(x=\frac{L_{p}}{2}\right)+\mathbf{R}_{\mathbf{f}} \overline{\mathbf{A}}^{(\mathrm{II})}
$$

in which the matrix, $\mathbf{R}_{\mathrm{f}}$, denotes the inertia contributions of attached resonators as

$$
\mathbf{R}_{\mathbf{f}}=\left[\begin{array}{cccccc}
0 & 0 & 0 & 0 & 0 & 0 \\
0 & 0 & 0 & 0 & 0 & 0 \\
0 & 0 & 0 & 0 & 0 & 0 \\
f_{r} e^{\frac{\lambda_{1} L_{p}}{2}} & f_{r} e^{\frac{\lambda_{2} L_{p}}{2}} & f_{r} e^{\frac{\lambda_{3} L_{p}}{2}} & f_{r} e^{\frac{\lambda_{4} L_{p}}{2}} & f_{r} e^{\frac{\lambda_{s} L_{p}}{2}} & f_{r} e^{\frac{\lambda_{6} L_{p}}{2}} \\
0 & 0 & 0 & 0 & 0 & 0 \\
0 & 0 & 0 & 0 & 0 & 0
\end{array}\right],
$$

with

$$
f_{r}=-\frac{k_{s}}{\left(\omega_{0} / \omega\right)^{2}-1},
$$


and $k_{s}$ represents the effective stiffness of the rubber disk, $\omega_{0}=\sqrt{\frac{k_{s}}{m_{r}}}$ denotes the resonant frequency of the mechanical resonator with $m_{r}$ being the weight of the metal cylinder.

Combining Eqs. (5.20) and (5.21), the physical quantities on the right edge of section II of the beam can be related to those on the left edge of section I of the beam as

$$
\mathbf{Y}_{\mathbf{p}}^{(\mathbf{I I})}\left(x=L_{p}\right)=\mathbf{B}^{(\mathbf{I I})}\left[\mathbf{B}^{(\mathbf{I})}\right]^{-1} \mathbf{Y}_{\mathbf{p}}^{(\mathbf{I})}(x=0)
$$

where

$$
\begin{aligned}
& \mathbf{B}^{(\mathbf{I})}=\left[\mathbf{B}_{\mathbf{p}}(x=0)+\mathbf{F}^{(\mathbf{I})}\right]\left[\mathbf{B}_{\mathbf{p}}\left(x=\frac{L_{p}}{2}\right)\right]^{-1}\left[\mathbf{B}_{\mathbf{p}}\left(x=\frac{L_{p}}{2}\right)+\mathbf{R}_{\mathbf{f}}\right]+\mathbf{F}^{(\mathbf{I I})}, \\
& \mathbf{B}^{(\mathbf{I I})}=\mathbf{F}^{(\mathbf{I})}\left[\mathbf{B}_{\mathbf{p}}\left(x=\frac{L_{p}}{2}\right)\right]^{-1}\left[\mathbf{B}_{\mathbf{p}}\left(x=\frac{L_{p}}{2}\right)+\mathbf{R}_{\mathbf{f}}\right]+\mathbf{B}_{\mathbf{p}}\left(x=L_{p}\right)+\mathbf{F}^{(\mathbf{I I})} .
\end{aligned}
$$

Similarly, the relationship of physical quantities between the left and right edges of section III of the beam can also be obtained as

$$
\mathbf{Y}_{\mathbf{b}}^{(\mathrm{III})}\left(x=L_{b}\right)=\mathbf{B}_{\mathbf{b}} \mathbf{D}_{\mathbf{b}} \mathbf{B}_{\mathbf{b}}^{-1} \mathbf{Y}_{\mathbf{b}}^{(\mathrm{III})}\left(x=L_{p}\right)
$$

where

$$
\mathbf{Y}_{\mathbf{b}}^{(\mathrm{III})}=\left[w^{(b)}(x) \frac{\partial w^{(b)}}{\partial x}(x) M^{(b)}(x) T^{(b)}(x) u_{0}^{(b)}(x) N^{(b)}(x)\right]^{\mathrm{T}}
$$


$\mathbf{B}_{\mathbf{b}}=\left[\begin{array}{cccccc}1 & 1 & 1 & 1 & 0 & 0 \\ \lambda_{1}^{(b)} & \lambda_{2}^{(b)} & \lambda_{3}^{(b)} & \lambda_{4}^{(b)} & 0 & 0 \\ I^{(b)}\left(\lambda_{1}^{(b)}\right)^{2} & I^{(b)}\left(\lambda_{2}^{(b)}\right)^{2} & I^{(b)}\left(\lambda_{3}^{(b)}\right)^{2} & I^{(b)}\left(\lambda_{4}^{(b)}\right)^{2} & 0 & 0 \\ I^{(b)}\left(\lambda_{1}^{(b)}\right)^{3} & I^{(b)}\left(\lambda_{2}^{(b)}\right)^{3} & I^{(b)}\left(\lambda_{3}^{(b)}\right)^{3} & I^{(b)}\left(\lambda_{4}^{(b)}\right)^{3} & 0 & 0 \\ 0 & 0 & 0 & 0 & 1 & 1 \\ 0 & 0 & 0 & 0 & K^{(b)} \lambda_{5}^{(b)} & K^{(b)} \lambda_{6}^{(b)}\end{array}\right]$,

and

$\mathbf{D}_{\mathbf{b}}=\left[\begin{array}{cccccc}e^{\lambda_{1}^{(b)}\left(L_{b}-L_{p}\right)} & 0 & 0 & 0 & 0 & 0 \\ 0 & e^{\lambda_{2}^{(b)}\left(L_{b}-L_{p}\right)} & 0 & 0 & 0 & 0 \\ 0 & 0 & e^{\lambda_{3}^{(b)}\left(L_{b}-L_{p}\right)} & 0 & 0 & 0 \\ 0 & 0 & 0 & e^{\lambda_{b}^{(b)}\left(L_{b}-L_{p}\right)} & 0 & 0 \\ 0 & 0 & 0 & 0 & e^{\lambda_{b}^{(b)}\left(L_{b}-L_{p}\right)} & 0 \\ 0 & 0 & 0 & 0 & 0 & e^{\lambda_{b}^{(b)}\left(L_{b}-L_{p}\right)}\end{array}\right]$.

The continuity conditions at the boundary between sections II and III of the beam must satisfy the relation

$\mathbf{Y}_{\mathbf{b}}^{(\text {III })}\left(x=L_{p}\right)=\mathbf{Y}_{\mathbf{p}}^{(\text {II })}\left(x=L_{p}\right)$

Combining Eqs. (5.22) - (5.24), the transfer matrix for the unit cell presented in Fig.

5-2(b) can be written as

$\mathbf{Y}_{\mathbf{b}}^{(\mathrm{III})}\left(x=L_{b}\right)=\mathbf{T}_{\mathbf{p b}} \mathbf{Y}_{\mathbf{p}}^{(\mathbf{I})}(x=0)$

with

$\mathbf{T}_{\mathrm{pb}}=\mathbf{B}_{\mathbf{b}} \mathbf{D}_{\mathbf{b}} \mathbf{B}_{\mathbf{b}}^{-1} \mathbf{B}^{(\mathrm{II})}\left[\mathbf{B}^{(\mathrm{I})}\right]^{-1}$. 
According to the Bloch theorem, the wave field $\mathbf{Y}_{\mathbf{b}}^{(\mathrm{III})}\left(x=L_{b}\right)$ and $\mathbf{Y}_{\mathbf{p}}^{(\mathbf{I})}(x=0)$ should be satisfied by

$$
\mathbf{Y}_{\mathbf{b}}^{(\mathrm{III})}\left(x=L_{b}\right)=e^{i k_{x} L_{b}} \mathbf{Y}_{\mathbf{p}}^{(\mathbf{I})}(x=0)
$$

where $k_{x}$ is the wavenumber along the $x$-direction. Comparing Eqs. (5.25) and (5.26), the dispersion relations can then be determined by solving the eigensystem given as

$$
\mathbf{T}_{\mathbf{p b}} \mathbf{Y}_{\mathbf{p}}^{(\mathbf{I})}(x=0)=e^{i k_{x} L_{b}} \mathbf{Y}_{\mathbf{p}}^{(\mathbf{I})}(x=0)
$$

It should be mentioned that complex wavenumbers for flexural and longitudinal modes can be found with a given frequency due to the coupling between the mechanical resonance and electrical shunting circuits, in which the real and imaginary parts of the wavenumber represent the wave propagation and attenuation constants, respectively. It should be mentioned that the numerical instability of the transfer matrix method is not observed in the calculations. Because the hybrid elastic metamaterial beam with only three sections is mainly focused on low-frequency wave applications at a subwavelength scale. However, when the lengths of each of the sections are comparable with the wavelength and/or the unit cell contains lots of sections, the hybrid matrix method needs to be adopted to overcome numerical instability issues.

\subsubsection{Effective properties of the hybrid metamaterial beam}

Using the analytical effective method based on global fields obtained in the previous section, the effective properties of the proposed hybrid metamaterial beam can now be determined. The underlying goal for developing the effective continuum theory of the metamaterial beam is to establish the relationship between the wave displacement fields 
and the global force and moment responses. Under the long-wavelength assumption, the effective mass density and bending stiffness of the hybrid metamaterial beam can be easily obtained by prescribing different boundary conditions.

To determine the effective mass density, the transverse harmonic wave field at the left $(x=0)$ and right $\left(x=L_{b}\right)$ boundaries of the metamaterial unit cell is prescribed by $W_{p r}$, where the rotational angle and longitudinal displacement is set to zero at these two boundaries. The harmonic problem is then solved using Eq. (5.25) with the frequency swept over the range of interest, from which the global transverse reaction forces at the two boundaries can be determined. Therefore, the effective mass density can be defined as

$$
\rho_{\text {eff }}=\frac{\left[T^{(b)}\left(x=L_{b}\right)+T^{(\mathrm{I})}(x=0)\right]}{\omega^{2} W_{p r} V_{\text {host }}}
$$

in which $T^{(\mathrm{I})}(x=0)$ and $T^{(b)}\left(x=L_{b}\right)$ represent the calculated global transverse reaction forces at the left and right boundaries of the unit cell, respectively, and $V_{\text {host }}$ denotes the volume of the host beam.

To determine the effective bending stiffness, the harmonic rotational angles at the left $(x=0)$ and right $\left(x=L_{b}\right)$ boundaries of the metamaterial unit cell are assumed as $-\varphi_{p r}$ and $\varphi_{p r}$, respectively. The transverse displacements are fixed at zero for both the boundaries. If longitudinal displacements are also constrained to zero, the global reaction bending moments at boundaries are required to be calculated with respect to the neutral axis of that beam to consider the coupling between the bending and longitudinal displacement. However, for the adaptive hybrid metamaterial beam proposed, the location 
of the neutral axis is dependent on the shunted negative capacitance, which is very difficult to be determined. Therefore, we release the longitudinal displacement at the boundaries on either one side or two sides (the longitudinal force is set to be zero) such that the global reaction bending moments on the boundaries can be naturally obtained with respect to the rotational center, where effects of the longitudinal displacement are decoupled. Specifically, the longitudinal displacement on the left boundary $(x=0)$ of the unit cell is fixed and the longitudinal force on the right boundary $\left(x=L_{b}\right)$ is set to be zero, where the obtained reaction bending moment with respect to the rotational center can be used for the effective bending stiffness calculation. After solving the harmonic problem, the effective bending stiffness of the hybrid metamaterial beam can be defined as

$$
D_{e f f}=\frac{M^{(b)}\left(x=L_{b}\right)}{\left(2 \varphi_{p r} / L_{b}\right)}
$$

where $M^{(b)}\left(x=L_{b}\right)$ represents the global reaction bending moment calculated at the right boundary of the unit cell.

\subsection{Results and discussion}

In this section, the effective mass density and effective bending stiffness of the adaptive hybrid metamaterial beam are first analytically calculated and validated by numerical simulations. Then, the dispersion curve of the adaptive hybrid metamaterial beam is investigated for its tunable wave manipulation properties and elucidated using the effective material parameters obtained. Finally, the tunable wave control capabilities are illustrated and examined by conducting multi-physical wave propagation simulations in the finite hybrid metamaterial beam and plates. 


\subsubsection{Effective material parameters of the metamaterial beam}

Effective properties of the passive metamaterial and adaptive phononic crystal are initially studied, which will be used as the foundation for conceiving the adaptive hybrid metamaterial system. Figure 5-3(a) shows the normalized effective mass density of a passive metamaterial beam (as shown in Fig. 5-1(a)) calculated analytically and numerically over frequencies of interest. In the study, the material and geometric parameters of the host beam are listed in Tab. 5-1, and the width is selected as $20 \mathrm{~mm}$. The spring constant and weight of the mechanical resonator in the model are selected as $1 \times 10^{5}$

$\mathrm{N} / \mathrm{m}$ and $5 \times 10^{-3} \mathrm{~kg}$, respectively. In the figure, the normalized frequency $\Omega$ is defined as $\omega^{\prime} \omega_{0}$, where $\omega_{0}$ is the resonant frequency of the mechanical resonator. The simulation results presented in Fig. 5-3 are performed using COMSOL Multiphysics utilizing the same 2D geometric and boundary conditions as those used in the analytical model. It can be observed from Fig. 5-3(a) that the analytical and numerical results are in excellent agreement, which confirms the validity of the effective mass density calculations in the analytical model. As expected, the normalized effective mass density of the passive metamaterial becomes negative at the normalized frequencies between 1 and 2 (shaded area), due to the out-of-phase motions of the resonator and host beam. It should be mentioned that the effective material parameter calculations in this study are based on the subwavelength assumption. Figure 5-3(b) shows the normalized effective bending stiffness of an adaptive phononic beam (as shown in Fig. 5-1(b)) with different uniformly shunted negative capacitances for an array of PZT-5H patches using both the analytical and numerical approaches at $10 \mathrm{~Hz}$. In the figure, $D_{0}$ denotes the bending stiffness of the host beam and the material and geometric parameters listed in Tab. 5-1. Both the analytical and 
numerical results demonstrate that the normalized effective bending stiffness can gradually decrease (soften) while approaching zero before becoming negative by decreasing the negative capacitance (shaded area). After the normalized effective bending stiffness passes through its pole, it will decrease from the extremely large value (hardening) to the open circuit value. Again, very reasonable agreement can be found where the minor difference for the pole position's prediction is caused by the relatively small unit cell length-tothickness ratio (which is 7.7 for the case in Fig. 5-3(b)), in which the Euler-beam assumption is still employed. It should be mentioned that, when the unit cell length-tothickness ratio is greater than 12 , the analytical and numerical results will have an excellent agreement according to our results. The bending stiffness variation due to the negative capacitance such as stiffness softening (even negative) and hardening will be harnessed for the design of the hybrid metamaterial.

Table 5-1. Geometric and material parameters of the passive locally resonant metamaterial beam and adaptive piezoelectric phononic beam.

\begin{tabular}{|c|c|c|c|}
\hline \multicolumn{4}{|c|}{ Geometric parameters (mm) } \\
\hline$\overline{L_{b}}$ & 20.0 & $L_{p}$ & 18.0 \\
\hline$h_{b}$ & 1.6 & $h_{p}$ & 1.0 \\
\hline \multicolumn{4}{|c|}{ Material properties (Host beam: Aluminum) } \\
\hline$E_{b}$ & $70.0 \mathrm{GPa}$ & $v_{b}$ & 0.33 \\
\hline$\rho_{b}$ & $2700.0 \mathrm{~kg} / \mathrm{m}_{3}$ & & \\
\hline \multicolumn{4}{|c|}{ Material properties (Piezoelectric patch: PZT-5H) } \\
\hline$c_{11}^{E}$ & $126.0 \mathrm{GPa}$ & $e_{15}$ & $17.0 \mathrm{C} / \mathrm{m}^{2}$ \\
\hline$c_{12}^{E}$ & $79.5 \mathrm{GPa}$ & $e_{31}$ & $-6.55 \mathrm{C} / \mathrm{m}^{2}$ \\
\hline$c_{13}{ }^{E}$ & $84.1 \mathrm{GPa}$ & $e_{33}$ & $23.3 \mathrm{C} / \mathrm{m}^{2}$ \\
\hline
\end{tabular}



$c_{33}{ }^{E}$
117.0 GPa
$\varepsilon_{11}^{S}$
$1700.0 \varepsilon_{0}$
$c_{44}{ }^{E}$
$23.0 \mathrm{GPa}$
$\varepsilon_{33}^{S}$
$1470.0 \varepsilon_{0}$
$c_{66}{ }^{E}$
23.25 GPa
$\rho_{p}$
$7600.0 \mathrm{~kg} / \mathrm{m}_{3}$

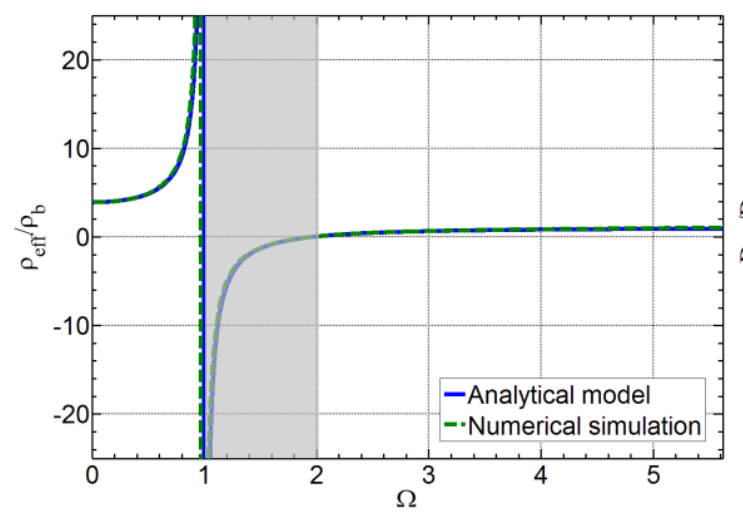

(a)

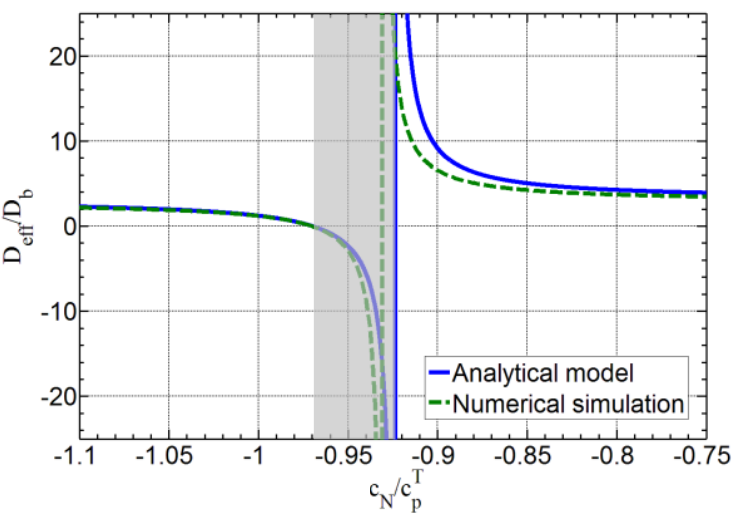

(b)

Figure 5-3 (a) Normalized effective mass density of the passive locally resonant metamaterial beam at different frequencies. (b) Normalized effective bending stiffness of the adaptive piezoelectric phononic beam with different negative capacitance shunting circuits

Figure 5-4 shows the normalized effective mass density and bending stiffness of the hybrid metamaterial (Fig. 5-1(c)) with short-circuit and three different negative capacitance circuits, which represent the softening, negative and hardening effective bending stiffness of the host medium based on the results presented in Fig. 5-3(b). In the study, the material and geometric parameters are the same as those used in Fig. 5-3 for the locally resonant metamaterial and piezoelectric phononic beam. As shown in Fig. 5-4(a) when the short circuit boundary condition is applied to the PZT patch, the negative effective mass density due to the out-of-phase motion of the resonator (shaded area) is still found but in a much 
narrower frequency range $(1.0 \sim 1.45)$ than that in Fig. $5-3(\mathrm{a})$. This is because the attached piezoelectric patch increases the weight of the host medium. Furthermore, it is interesting to note that the effective bending stiffness is almost constant over most of the frequency region but can be significantly affected by the transverse motion of the resonator when the frequency is close to the resonant frequency. As illustrated in the figure, when the frequency approaches the resonant frequency, the effective bending stiffness of the metamaterial will dramatically decrease to negative values in a very narrow frequency range (shaded area), then jump to large positive values after passing by the resonant frequency. After that, the effective bending stiffness will return rapidly to a constant value. The mechanism for forming the negative bending stiffness will be studied in greater detail later by conducting wave mode analysis. Figure 5-4(b) shows the effective property variation of the hybrid metamaterial for the case of stiffness softening of the shunted piezoelectric patch where the negative capacitance is selected as $c_{N} / c_{p}^{T}=-0.971$. By comparing the results in Figs. 5-4(a) and (b), it can be found that both the magnitude and the shape of the normalized effective mass density is not very sensitive to the change of the effective stiffness. This is because the effective mass density is primarily caused by the local transverse motion of the resonator. However, the normalized effective bending stiffness of the hybrid metamaterial is significantly reduced by simply "shearing" the effective bending stiffness to a lower value, compared with results in Fig. 5-4(a). As a result, the normalized frequency region of the negative effective bending stiffness induced by the resonant motion (shaded area at lower frequencies) is enlarged to $0.82 \sim 1.0$, and another negative effective bending stiffness region is found at normalized frequencies higher than 2.7 (shaded area at higher frequencies). However, the frequency regions of the 
negative effective mass and the negative effective bending stiffness do not overlap. Figure 5-4(c) shows the effective properties of the hybrid metamaterial for the case of the negative stiffness of the shunted piezoelectric patch where the negative capacitance is selected to be $c_{N} / c_{p}^{T}=-0.95$. As expected, the effective mass density is the same as the results shown Figs. 5-4(a) and (b). However, the effective bending stiffness is shifted to further negative values at almost all frequencies (shaded areas). As a result, the frequency region of negative effective mass is now completely immersed in the broad frequency region of the negative effective bending stiffness. Therefore, the double negative material parameters of the hybrid metamaterial are achieved over the broadest range of frequencies. Figure 5-4(d) shows the effective properties of the hybrid metamaterial for the case of stiffness hardening where the negative capacitance is selected to be $c_{N} / c_{p}^{T}=-0.9225$. In this case, the effective bending stiffness becomes a very large positive value at almost all frequencies. More specifically, the normalized effective mass density and bending stiffness as functions of both frequency and negative capacitance values are quantitatively illustrated in Fig. 55. The color bars in the figure denote the values of normalized effective material parameters. As shown in Fig. 5-5(a), the negative effective mass density frequency region is not sensitive to the change of the negative capacitances. However, the normalized effective bending stiffness is primarily influenced by the negative capacitance, and will be affected by the resonator when the frequency is close to the resonant frequency. By properly harnessing electric and mechanical elements, the proposed hybrid metamaterial would possess different material properties by combining the negative effective mass density with extremely soft, hard and even negative effective bending stiffness, respectively. 


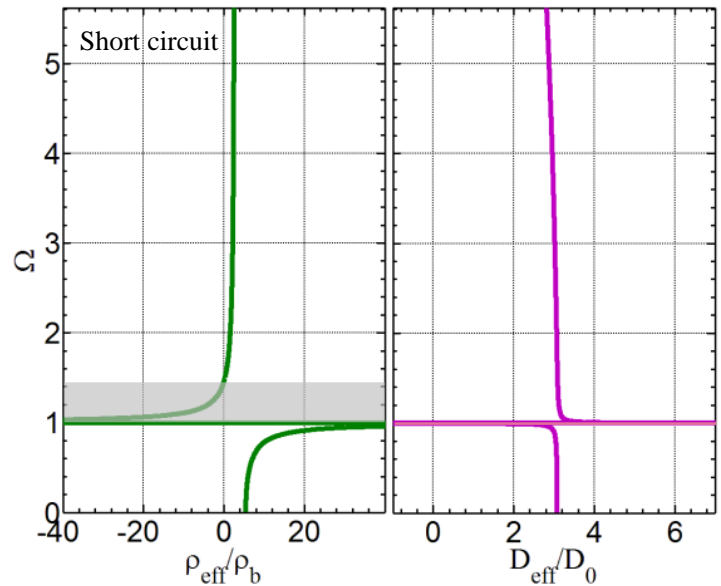

(a)

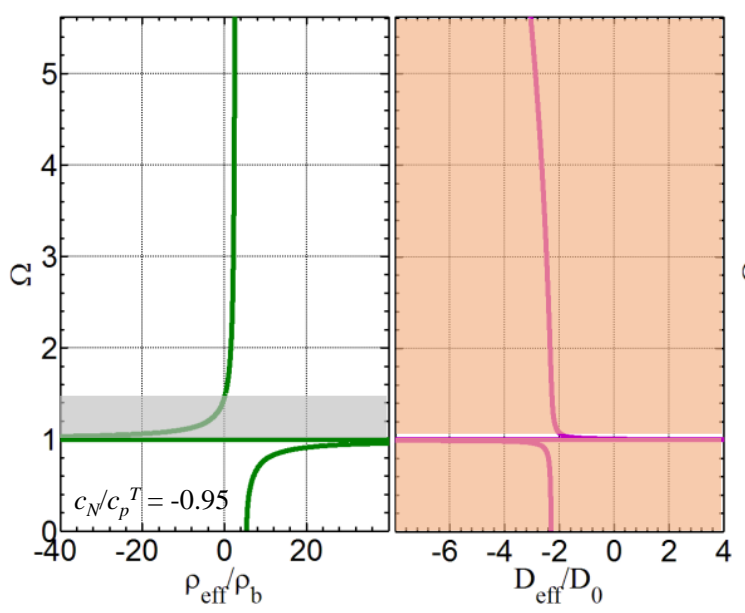

(c)

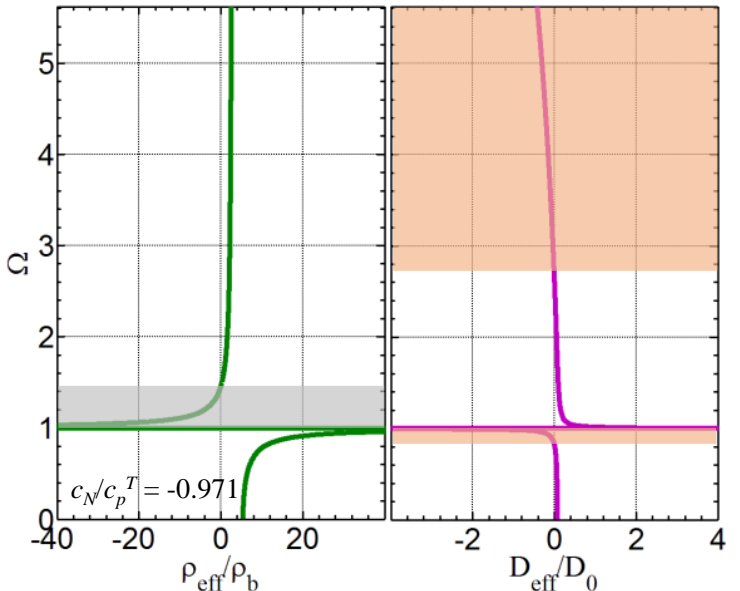

(b)

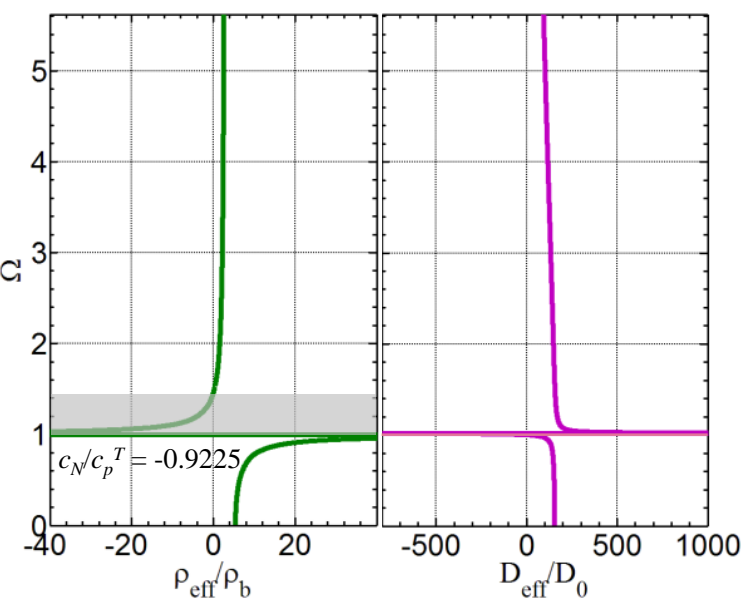

(b)

Figure 5-4 Normalized effective mass density and bending stiffness of the adaptive hybrid metamaterial beam with different negative capacitance shunting circuits: (a) short circuit, (b) $c_{N} / c_{p}^{T}=-0.971$, (c) $c_{N} / c_{p}^{T}=-0.95$, (d) $c_{N} / c_{p}^{T}=-0.9225$ 


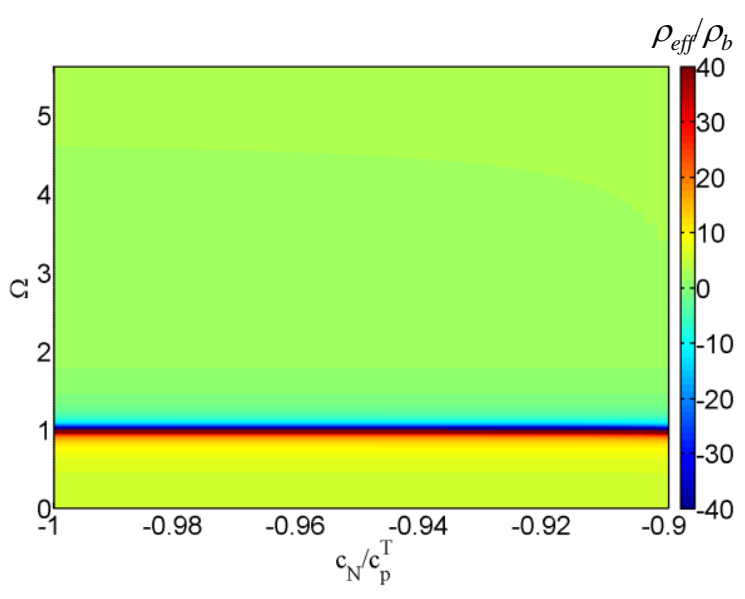

(a)

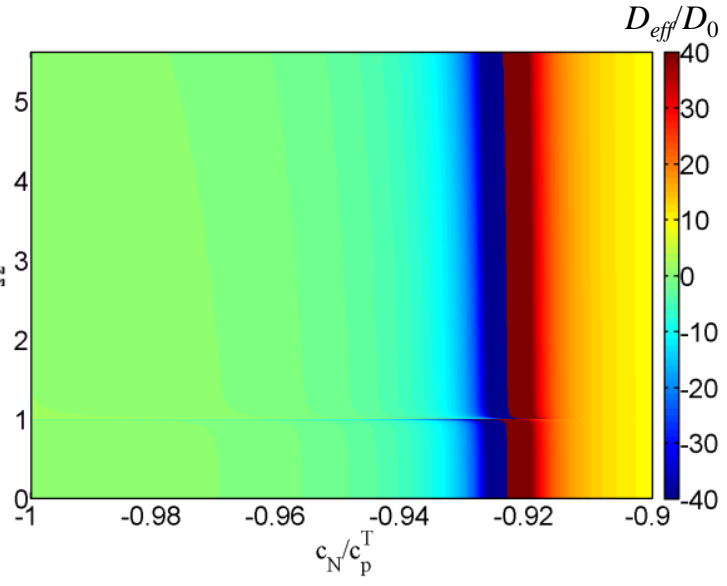

(b)

Figure 5-5 Normalized effective material parameters of the adaptive hybrid metamaterial beam in functions of the frequency and negative capacitance: (a) Normalized effective mass density, (b) Normalized effective bending stiffness

To further elaborate and understand the bending stiffness variations around the resonant frequency shown in Fig. 5-4(a), Figure 5-6 shows the mode shapes of the adaptive hybrid metamaterial beam at different frequencies, where material and geometric parameters are the same as those used in Fig. 5-4(a). In Fig. 5-6, the color bar represents the normal stress along the $x$-direction, and the motion of the resonator is also qualitatively illustrated, which is not proportional to that of the host beam. As shown in Fig. 5-6(a), when the normalized frequency is selected to be 0.14 , which is far from the resonant frequency, the relative displacement between the resonator and the host beam is small, and the reaction force in the spring is very weak. Thus, the resonator would not affect the bending stiffness of the original beam. However, when the normalized frequency is increased to 0.993 (Fig. 5-6(b)) which approaches the resonant frequency, the resonator will move in-phase with the corresponding point on the host beam, and the relative displacement and force in the spring 
will become larger than that in Fig. 5-6(a). As a consequence, the bending deformation in the unit cell is mainly balanced by the increased resonant force and the reaction bending moments at the boundaries (the external applied bending moments at boundaries to maintain the deformation) is reduced. Therefore, the effective bending stiffness is essentially softened. By increasing the normalized frequency further to 0.996 (Fig. 5-6(c)) which is very close to the resonant frequency, the relative displacement and force in the spring become extremely large. The reaction bending moments are therefore required to be opposite to maintain the prescribed deformation at the boundaries. As a result, the effective bending stiffness becomes negative. However, when the normalized frequency is increased to 1.0003 (Fig. 5-6(d)) which is just above the resonant frequency, the resonator moves out-of-phase to the corresponding point on the host beam, and produces a large opposing force on the host beam. Thus, the curvature of the host beam becomes smaller or even opposite, and large in-phase reaction bending moments at the boundaries of the host beam will induce a large positive effective bending stiffness.

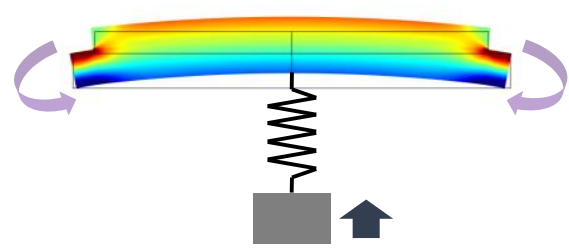

(a)

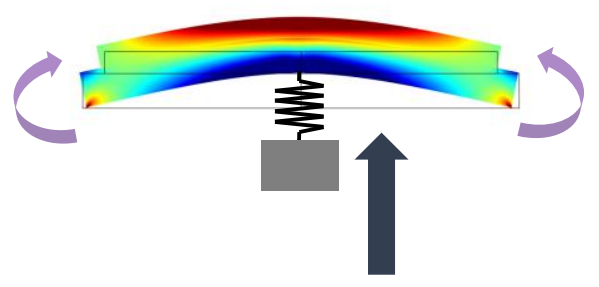

(c)

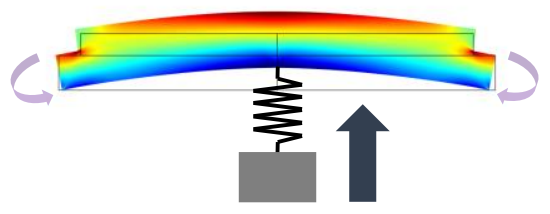

(b)

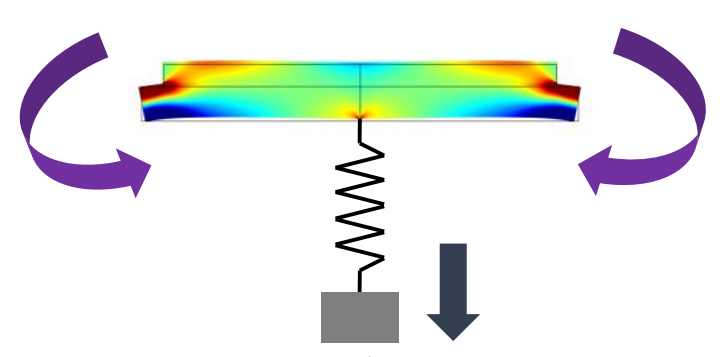

(d)

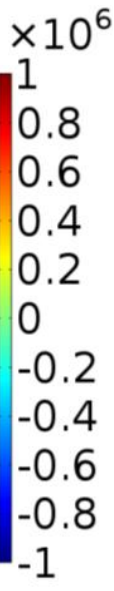

0.8 0.6 0.4

0.2 $-0.2$ $-0.4$ $-0.6$ 0.8 
Figure 5-6 Mode shapes of the adaptive hybrid metamaterial beam at different normalized frequencies: (a) $\Omega=0.14$, (b) $\Omega=0.993$, (c) $\Omega=0.996$, (d) $\Omega=1.0003$

\subsubsection{Tunable wave dispersion analysis of the metamaterial beam}

Figure 5-7 shows the complex band structures for flexural waves in the hybrid metamaterial with short-circuit and three different negative capacitance circuits which represent the bending stiffness softening, hardening and becoming negative as discussed in Fig. 5-4. The material, geometric and negative capacitance parameters are also left unchanged from those used in Fig. 5-4. As shown in Fig. 5-7(a) when the hybrid metamaterial is connected to a short-circuit, the imaginary part of the wavenumber in the complex band is not equal to zero at normalized frequencies from 1.0 to 1.45 (shaded area) indicating a complete band gap frequency region. In this case, the hybrid metamaterial behaves like a conventional passive metamaterial based on the local resonance mechanism. To understand the wave attenuation mechanism, the resulting subwavelength band gap frequency region is also compared with the frequency-dependent effective mass density and bending stiffness of the hybrid metamaterial beam shown in Fig. 5-4(a). It is found the band gap behavior is mainly caused by the negative mass density due to the out-of-phase motion of the mechanical resonator (shaded area). Figure 5-7(b) shows the complex band structure of the hybrid metamaterial for the softened bending stiffness where the negative

capacitance is selected to be $c_{N} / c_{p}^{T}=-0.971$. As shown in the figure, besides the local resonance band gap, two subwavelength Bragg band gaps are found below and above the resonant band gap (shaded areas), with the normalized frequencies from 0.83 and 2.7, respectively. It should be mentioned that the lower Bragg band gap in the complex band structure is combined with the resonant band gap forming an entirely new band gap region 
(shaded area), which significantly enhances the wave attenuation at much lower frequencies. In addition, the attenuation constant at the resonant band gap is much larger than in case of the short-circuit metamaterial (Fig. 5-4(a)). Compared with the frequencydependent effective mass density and bending stiffness of the hybrid metamaterial beam shown in Fig. 5-4(b), it is found the resonant band gap is still caused by the negative effective mass density due to the out-of-phase motion of the resonator (shaded area in Fig. 5-4(b)). It should be noticed that the local resonance induces not only a negative effective mass but also an extremely large mass just below the resonant frequency, due to the inphase motion of the resonator. The large mass results in strong spatial oscillation of wave fields within the periodic structures, giving rise to the Bragg gap. This low-frequency Bragg gap might be different from the common one at high-frequencies because the Bragg resonance is occurring in the sub-wavelength scale, and it is related to the local resonances in the metamaterial. This directional low frequency Bragg band gap induced by the local resonance has also been discussed in other literatures. Figure 5-7(c) shows the complex band structure of the hybrid metamaterial for the negative bending stiffness where the negative capacitance is selected to be $c_{N} / c_{p}^{T}=-0.95$. The resulting stop bands occupy almost the entire range of frequencies (shaded area) and a passing band with negative phase velocity is observed between the two stop bands. In the two stop band regions, the magnitude of the real and imaginary parts of the wavenumber are almost identical due to the solution feature of the bi-harmonic governing equation of the flexural wave. To explain the wave behavior, the complex band structure is also compared with the effective mass density and bending stiffness predicted in Fig. 5-4(c). It is found the wave attenuation in the two stop bands is caused by the negative effective bending stiffness of the hybrid 
metamaterial, and the pass band with negative refraction is due to simultaneously negative effective mass density and bending stiffness. It can be observed that the frequency region of the negative refractive index is almost the same as the frequency region of the negative mass density, which cannot be produced in any passive double negative metamaterial because the frequency region of the negative modulus band is usually very narrow compared with that of the negative mass density. Figure 5-7(d) shows the complex band structure of the hybrid metamaterial with an extremely positive bending stiffness where the negative capacitance is selected to be $c_{N} / c_{p}^{T}=-0.9225$. Although the complex band structure is similar to that shown in Fig. 5-7(a) for the metamaterial with short circuits, the magnitude of the imaginary part of the wavenumber (attenuation constant) is significantly smaller than that in Fig. 5-7(a) at the normalized frequencies from 1.0 to 1.45 (shaded area). This indicates that the wave attenuation and/or stop band due to both the resonant motion and Bragg scattering mechanisms has nearly vanished or been switched OFF by the stiffness hardening shown in Fig. 5-4(d).

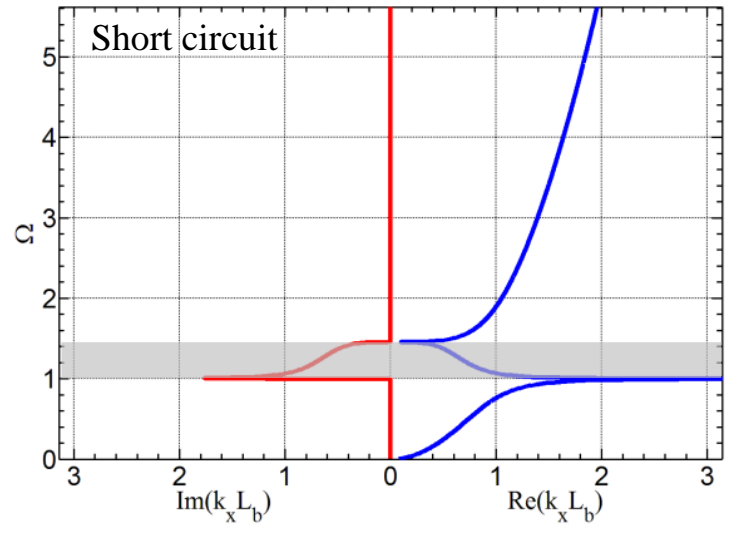

(a)

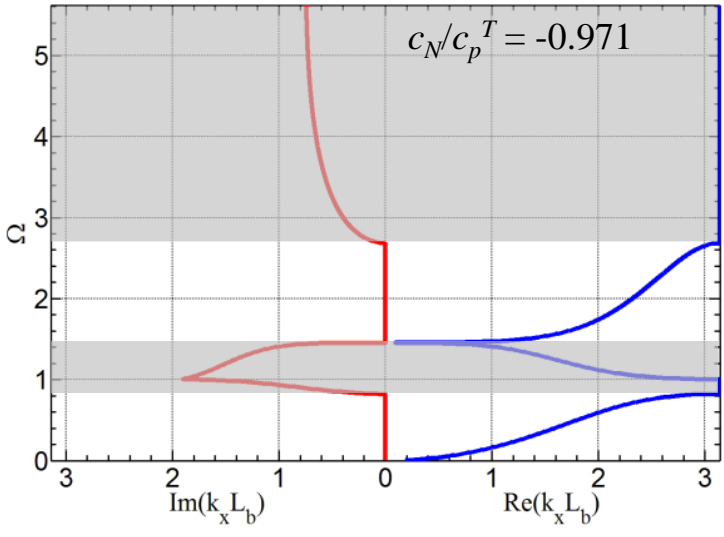

(b) 


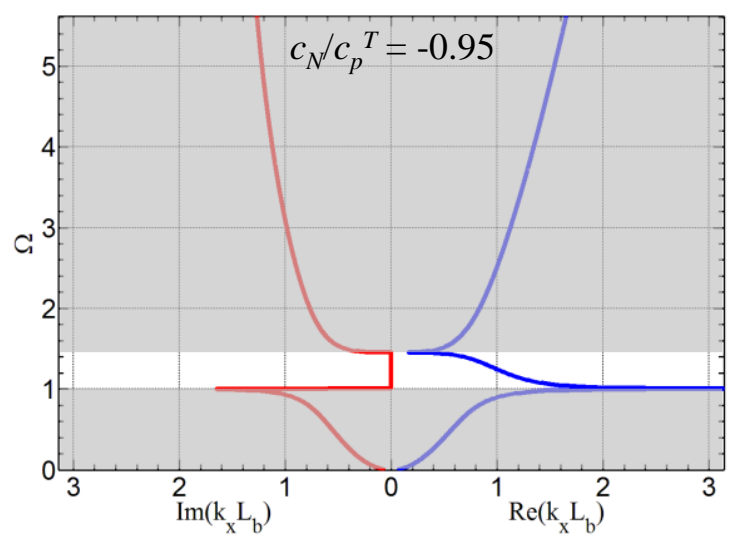

(c)

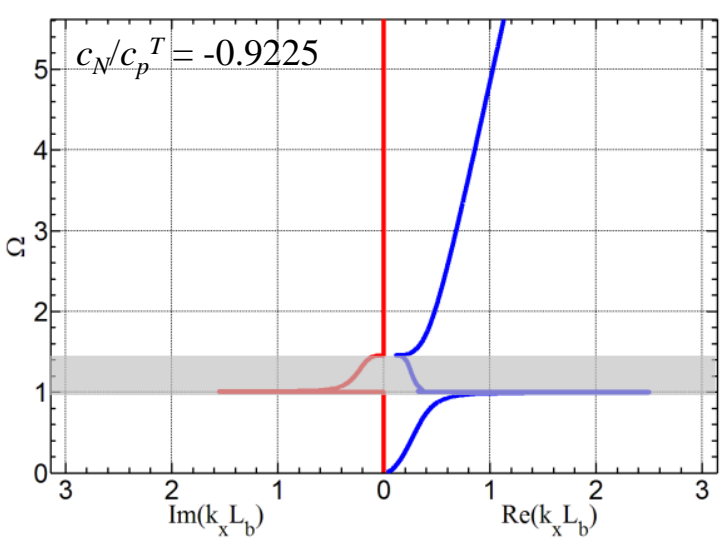

(d)

Figure 5-7 Complex band structures of flexural waves in the adaptive hybrid metamaterial beam with different negative capacitance shunting circuits: (a) short circuit,

$$
\text { (b) } c_{N} / c_{p}^{T}=-0.971 \text {, (c) } c_{N} / c_{p}^{T}=-0.95 \text {, (d) } c_{N} / c_{p}^{T}=-0.9225
$$

To characterize the wave propagation behavior, complex wave dispersion relations of the hybrid metamaterial are plotted in Fig. 5-8 as a function of both the wave frequency and negative capacitance. The color bars in the figure denotes the values of the real and imaginary parts of the complex wavenumber. Based on the contour value of the wavenumber, the unusual and tunable wave attenuation/manipulation properties controlled by the negative capacitance can be artificially classified into five regions. Typically, in regions 1 and 5, the adaptive hybrid metamaterial beam exhibits a resonant-based band gap behavior as that in the passive metamaterial beam, where waves with normalized frequency between 1.0 and 1.45 will be attenuated. In the region 2 , the resonant-based band gap is significantly enhanced by coupling with the lower-frequency Bragg band gap. However, the wave characterization in the region 3 is almost opposite to those in the regions 1 and 5 where a pass band is apparent in the original stop band frequency region, and the original pass bands are switched to stop bands. The most important aspect is that the broadest 
frequency region of a negative refractive index is found in this region. Finally, in region 4 the wave attenuation ability of the hybrid metamaterial is almost entirely switched OFF. The wave manipulation characteristics in the five regions will be further examined with 3D numerical simulations in the following section.

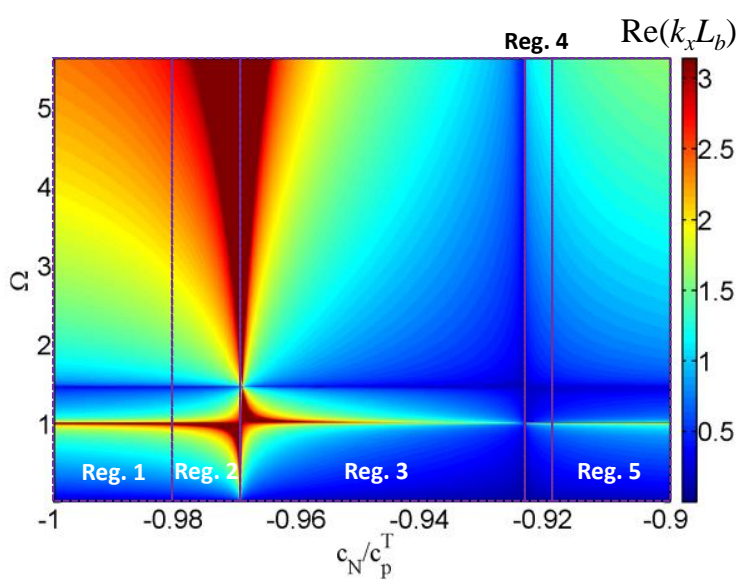

(a)

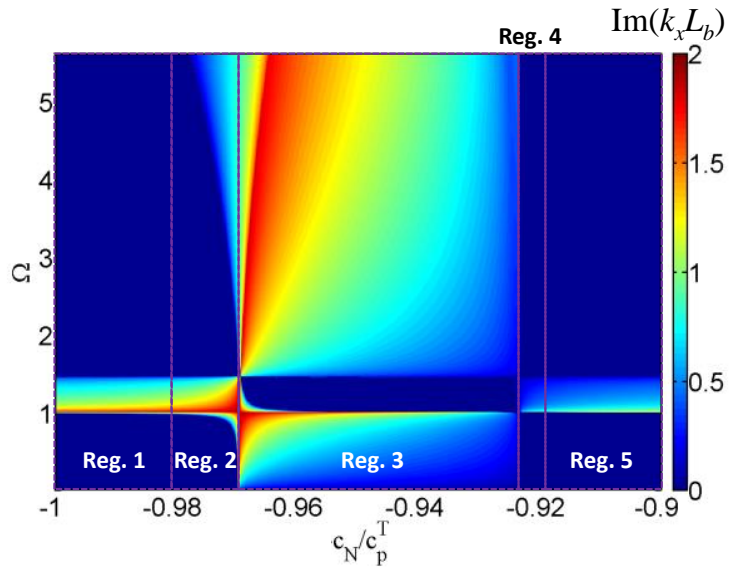

(b)

Figure 5-8. Complex flexural wave dispersion relations of the adaptive hybrid metamaterial beam in function of the frequency and negative capacitance: (a) Real part of the wavenumber, (b) Imaginary part of the wavenumber

\subsubsection{Tunable wave transmittance of a finite metamaterial beam}

In order to examine and validate the tunable wave transmission properties of the proposed adaptive hybrid metamaterial controlled by different negative capacitances as illustrated in Figs. 5-7 and 5-8, a 3D multi-physical numerical simulation of the harmonic wave transmission in the finite hybrid metamaterial is conducted. The schematic of the numerical modeling is shown in Fig. 5-9(a). In the simulation, the 3D linear piezoelectric constitutive law is applied to describe dynamic behaviors of the piezoelectric patch by using the commercial finite element software COMSOL Multiphysics. The piezoelectric 
shunting boundary condition is implemented through a weak form expression. The incident flexural wave is generated by applying a harmonic voltage across a PZT patch bonded to the host beam on the left-hand side of the metamaterial. Two perfectly-matched layers (PMLs) are attached to both ends of the host beam in order to suppress reflected waves from the boundaries. A displacement probe is defined on the host beam in the right-hand side of the metamaterial region to measure the out-of-plane displacement and calculate the wave transmittance. As shown Fig. 5-9(a), the height of the resonant metal cylinder and the rubber disk are represented by $\mathrm{hm}$ and $\mathrm{hr}$, respectively, and the diameters of the metal cylinder and rubber disk are selected to be the same value and denoted by $\mathrm{dm}$. The materials of the host beam and piezoelectric patches used in the simulation are the same as those in used the analytical model. Other material and geometric parameters are given in Tab. 5-2.

Table 5-2. Geometric and material parameters of the proposed adaptive hybrid metamaterial beam

Geometric parameters (mm)

\begin{tabular}{cccc}
\hline$L_{b}$ & 7.0 & $L_{p}$ & 6.4 \\
$h_{b}$ & 1.6 & $h_{p}$ & 1.0 \\
$d_{m}$ & 4.0 & $h_{m}$ & 2.5 \\
$h_{r}$ & 1.0 & \\
\hline \multicolumn{4}{c}{ Material properties (Stub resonator: Steel) } \\
\hline$E_{s}$ & $210.0 \mathrm{GPa}$ & 0.3 \\
$\rho_{s}$ & $7650.0 \mathrm{~kg} / \mathrm{m}_{3}$ & \\
\hline$E_{r}$ & Material properties (Disk: Rubber) & \\
\hline$\rho_{r}$ & $25.0 \mathrm{MPa}$ & \\
\hline
\end{tabular}


Based on the numerically calculated effective bending stiffness, the normalized negative capacitances for tunable wave manipulation demonstrated in regions $2-4$ can be easily determined as $c_{N} / c_{p}^{T}=-0.9330,-0.9140$ and -0.9105 , respectively, which represent the softening, negative and hardening bending stiffness. Figure 5-9(b) shows the transmittance of the finite adaptive hybrid metamaterial with ten unit cells shunted with short and the three negative capacitance circuits. For the case of a short shunting circuit, a sharp wave transmission dip from 7.3 to $8.8 \mathrm{kHz}$ (shaded area) is found, which will be used as the basis for the tunable wave control. It can be found that (1) the original wave attenuation region is enlarged to $7.1 \sim 8.8 \mathrm{kHz}$ at lower frequencies when the normalized negative capacitance, $c_{N} / c_{p}^{T}$, is -0.933 (stiffness softening); (2) the wave characterization is almost reversed when the normalized negative capacitance, $c_{N} / c_{p}^{T}$, is -0.914 (negative stiffness) such that the original stop band is switched to a pass band with the negative phase velocity, and the original pass bands are switched to stop bands; (3) the wave attenuation due to the local resonator is almost entirely switched OFF when the normalized negative capacitance, $c_{N} / c_{p}^{T}$, is -0.9105 (stiffness hardening) and the transmittance is nearly uniform at all frequencies with some fluctuations in the original wave attenuation region (shaded area). Compared with the wave transmittance illustrated in the first and third figures in Fig. 5-9(b), we also notice that the wave attenuation due to the negative bending stiffness is much weaker than that from the negative mass density. 


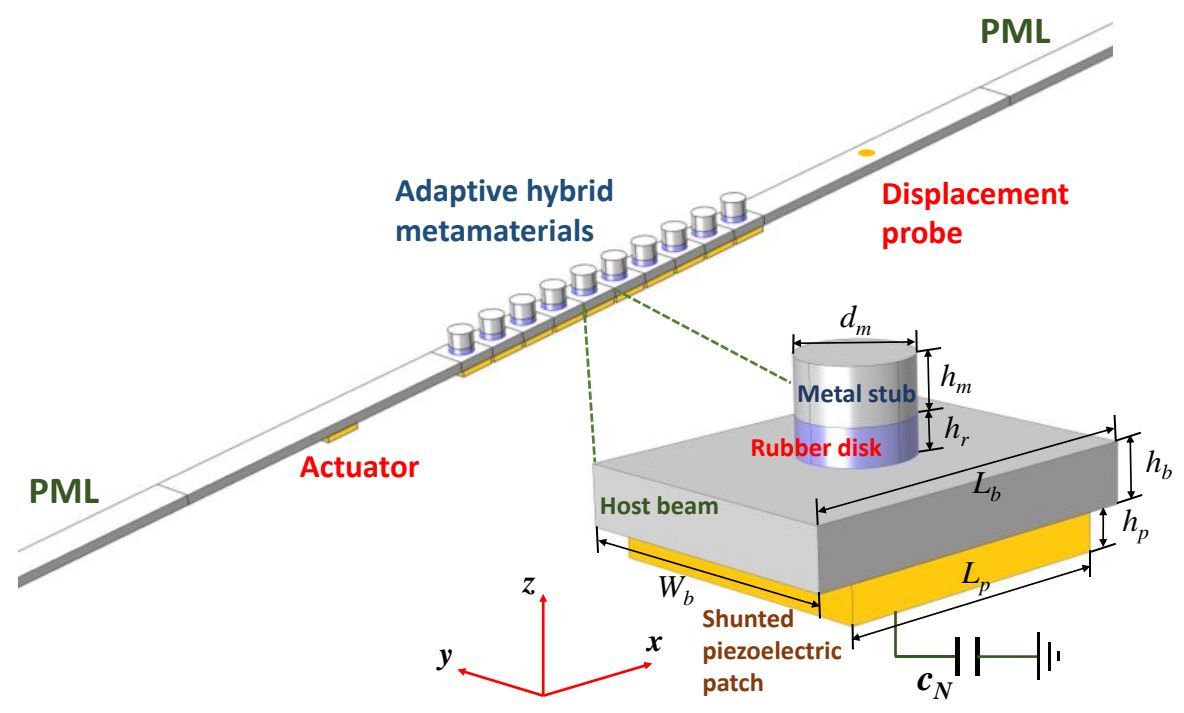

(a)

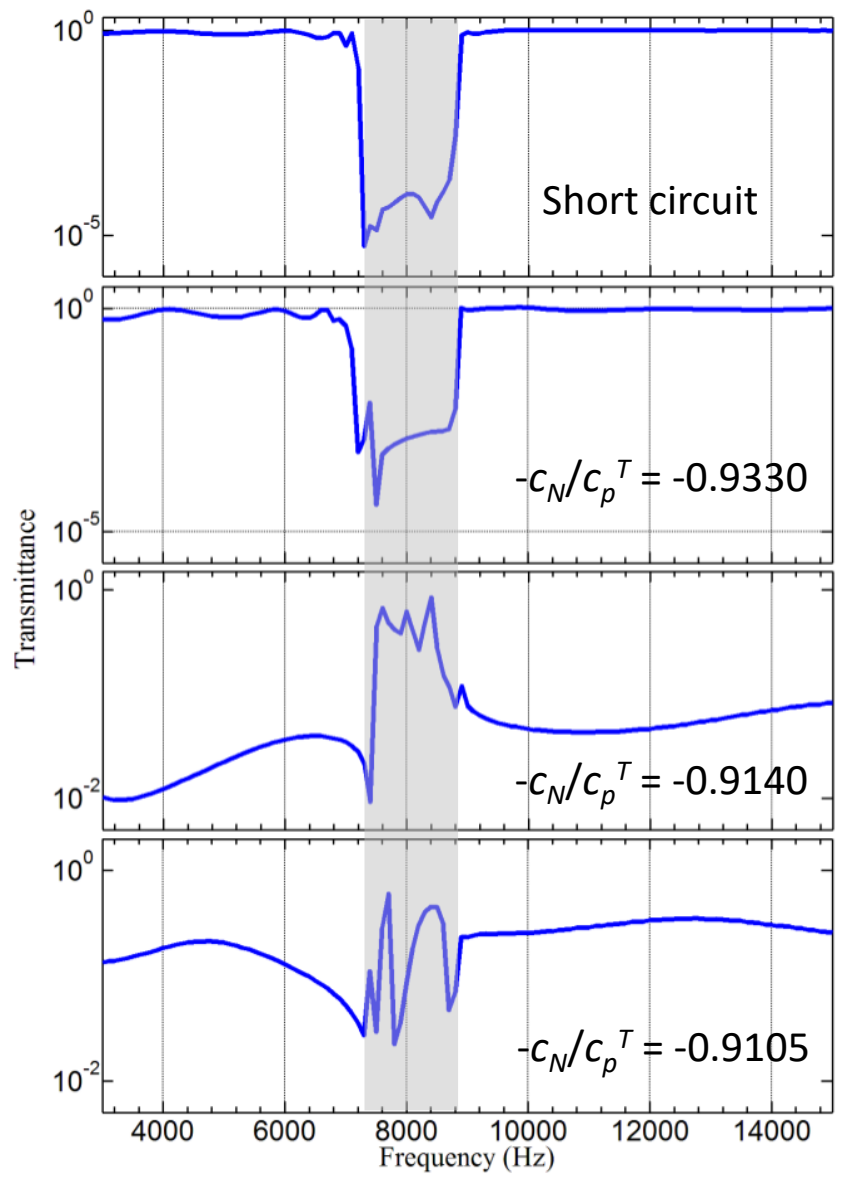

(b) 
Figure 5-9 (a) Schematic of 3D numerical simulations of the flexural wave transmission

in a finite adaptive hybrid metamaterial beam. (b) Transmittance of flexural waves through an adaptive hybrid metamaterial beam with 10 unit cells with short and different negative capacitance shunting circuits

\subsubsection{Broadband negative refraction of a metamaterial plate}

Based on the mechanism and wave behavior demonstrated in the metamaterial beam structure, the proposed adaptive hybrid metamaterial is extended into plate structures for tunable wave manipulations. Due to the broadband double negativities (negative mass density and negative bending stiffness) of the proposed metamaterial, broadband negative refraction in the entire negative mass density frequency region will be expected in the hybrid metamaterial plate. To examine this phenomenon, numerical simulations of a flat negative refraction lens composed of an array of $5 \times 48$ unit cell hybrid metamaterial plate are conducted at different frequencies. As the schematic shown in Fig. 5-10(a), the negative refraction lens is inclined $45^{\circ}$ to the lower edge of the host plate, and a Gaussian flexural wave beam is incident from the left edge of the host plate to the lower part of the lens with the actuation length being $150 \mathrm{~mm}$. In the harmonic numerical simulation, the PML layers are adopted to suppress reflected waves from the boundaries. The width of the host plate unit cell, $w_{b}$, is selected to be the same with $L_{b}$, and all the other geometric and material parameters are the same as those used in Fig. 5-9.

Figures 5-10(b), (c) and (d) show the normalized out-of-plane displacement wave fields

of the metamaterial plate with negative effective bending stiffness $\left(c_{N} / c_{p}^{T}=-0.871\right)$ at 7400, 7800, and $8200 \mathrm{~Hz}$, respectively. As illustrated in Fig. 5-10(b), the negative refraction is observed at the two boundaries between the metamaterial and the host plate, 
due to the double negative material parameters at this frequency. As a result, the incident wave beam is shifted upward and propagates through the lens along the incident direction, which can be explained by applying the Snell's law twice. It should be mentioned that the wave reflection at metamaterial boundaries is quite small, as the refractive index of the metamaterial is close to the index of the host plate. The similar negative refraction phenomena are also demonstrated in Figs. 5-10(c) and (d). It can also be observed that with the increase of the operation frequency, the negative refractive angle becomes larger. This is because the metamaterial effective refractive index is enhanced with the decreased effective mass density and the effective bending stiffness remains being constant when the frequency is increased.

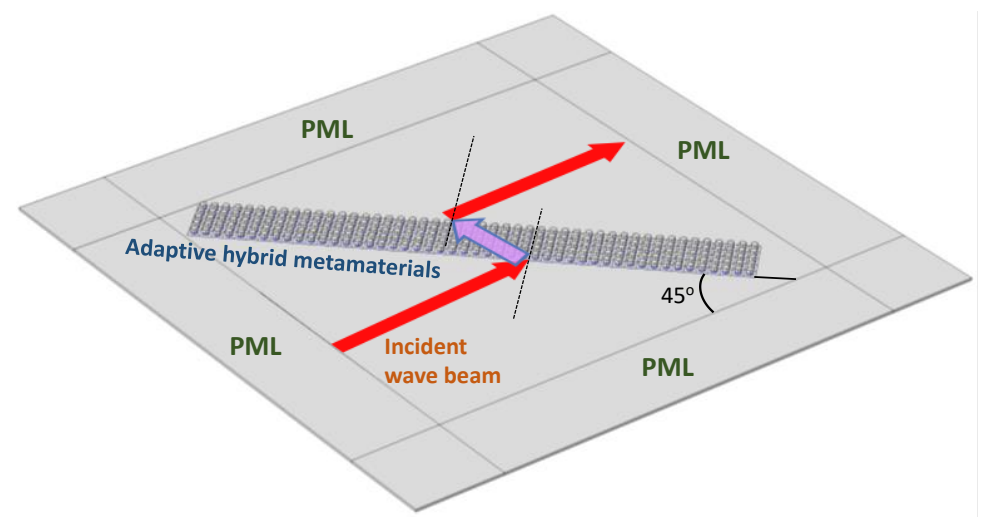

(a)
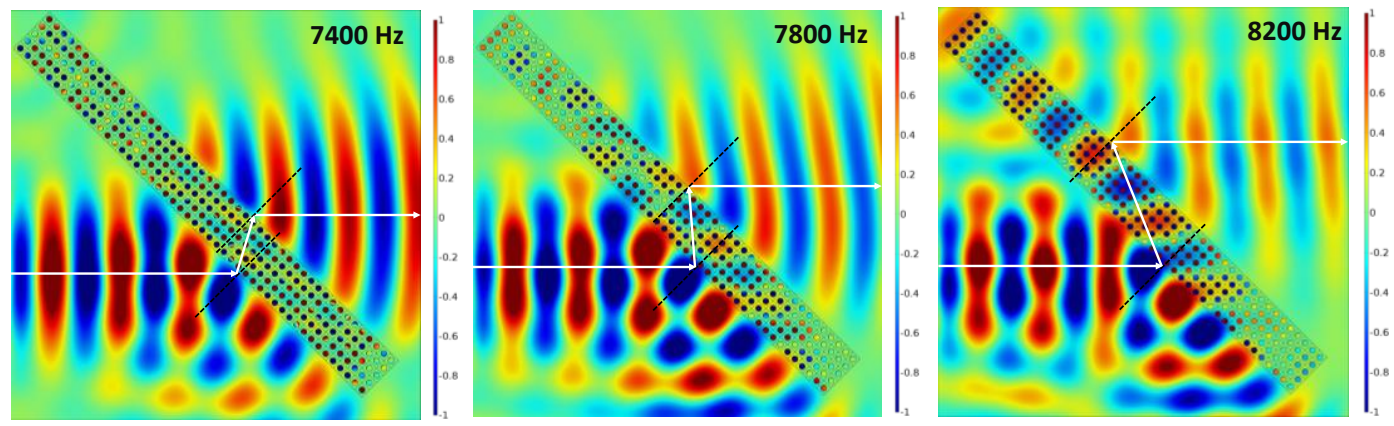

(b)

(c)

(d) 
Figure 5-10 (a) Schematic of 3D numerical simulations of the negative refraction in an adaptive hybrid metamaterial plate. (b-d) Normalized out-of-plane displacement fields of the adaptive hybrid metamaterial plate at different frequencies: (b) $7400 \mathrm{~Hz}$, (c) $7800 \mathrm{~Hz}$, (d) $8200 \mathrm{~Hz}$

\subsubsection{Programmed waveguiding of a metamaterial plate}

The proposed adaptive hybrid metamaterial can also be designed to implement with some digital control systems to switch circuits of unit cells between ON and OFF states to the metamaterial and achieve arbitrary waveguiding in a programmed manner. To demonstrate this application, Figure 5-11(a) shows the schematic of numerical simulations of the metamaterial-based waveguide with an array of $15 \times 15$ unit cells. The material and geometric parameters are the same as those used in Fig. 5-10. The inset figure shows the control map of the programmable waveguide, in which the colored region denotes the corresponding unit cells with negative capacitance circuits and the uncolored region denotes unit cells with short circuits. Therefore, the wave with frequency components between 7.3 to $8.8 \mathrm{kHz}$ can propagate through the colored region with negative phase velocity, while this wave is prohibit to travel through the uncolored region due to the negative mass density. As a result, the wave can only propagate followed the programmed L-shape waveguide (colored region) at those frequencies. Figures 5-11(b), (c) and (d) illustrate performances of the programmed waveguide with different shapes at a selected frequency, $8000 \mathrm{~Hz}$. The normalized out-of-plane displacement fields in the figures are coincided with the designed waveguide for wave steering angles smaller, equal and larger than $90^{\circ}$. It should be mentioned that the effective parameters would be controlled by tuning different circuit connection embedded inside the metamaterial structure. With such 
unique capabilities, physically realizable waveguide at different frequencies can be programmably achieved by using digital circuits and objects treated with these active metamaterials can even become acoustically invisible.

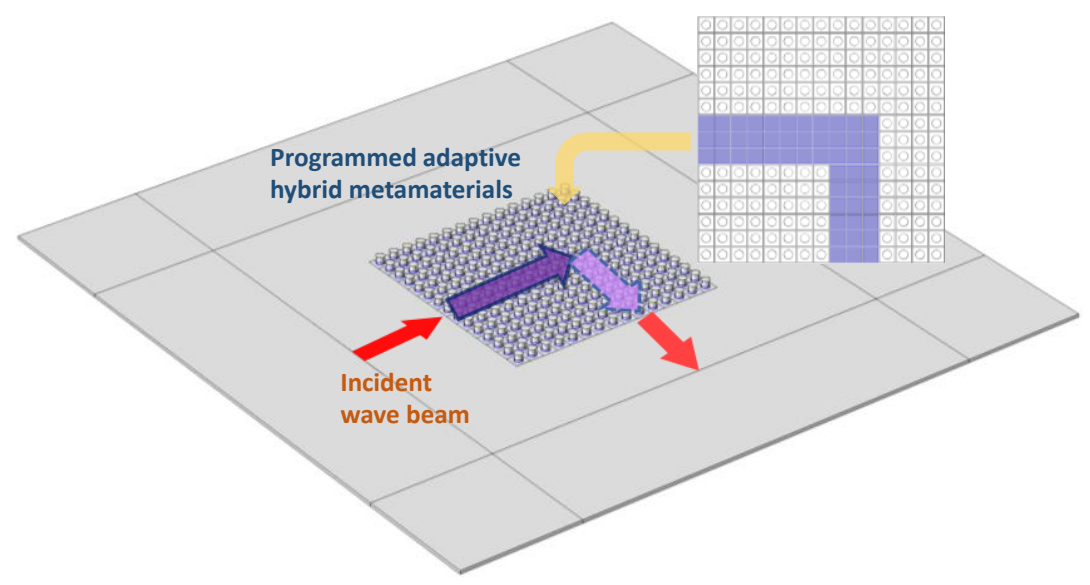

(a)

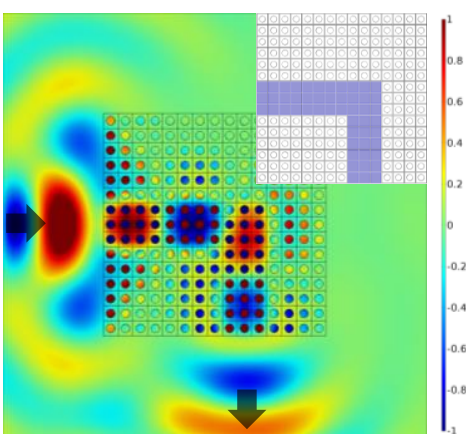

(b)

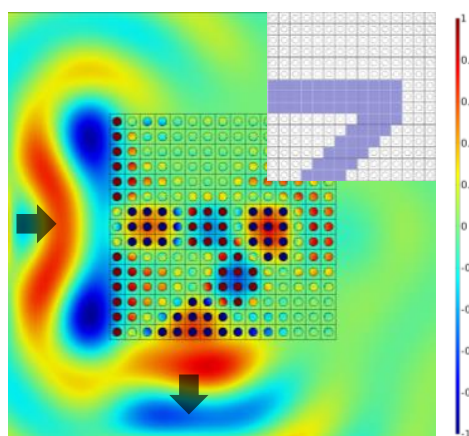

(c)

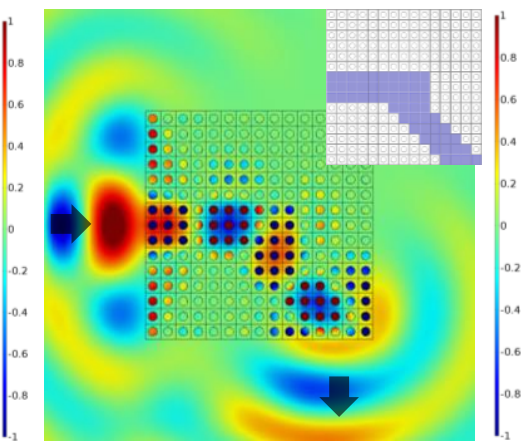

(d)

Figure 5-11 (a) Schematic of 3D numerical simulations of programmed waveguiding in an adaptive hybrid metamaterial plate. (b-d) Normalized out-of-plane displacement fields of the adaptive hybrid metamaterial plate with different control maps: (b) Shape-I, (c) Shape-II, (d) Shape-III 


\subsection{Conclusions}

In this chapter, we propose an adaptive hybrid metamaterial design with negative effective mass density and extremely tunable bending stiffness by bonding an array of negative capacitance shunted piezoelectric patches into a passive locally resonant metamaterial. First, effective material parameters as well as wave dispersion relations of the hybrid metamaterial beam are derived analytically based on the developed multiphysical model which considers the locally resonant motions, electro-mechanical coupling and shunting circuitry effects. Extremely tunable wave manipulation properties of the adaptive hybrid metamaterial are interpreted by combining the negative effective mass density and extremely tunable bending stiffness. Finally, 3D numerical simulations for wave transmission, broadband negative refraction and programmed waveguiding in the hybrid metamaterial are conducted. We hope that the proposed concept, design and approach can open many possibilities for tunable broadband wave/vibration attenuation, wave guiding and imaging applications, such as nondestructive evaluation and structural health monitoring. 


\section{Chapter 6 An Active Elastic Metamaterials for the Trajectory}

\section{Control of Flexural Waves}

\subsection{Introduction}

A transformation method has been initially proposed for controlling electromagnetic (EM) waves and determining the relation between the functionality of a device and the material pattern in the space [80]. Because of the similarity between the EM wave and the acoustic wave, the transformation method has also been proposed for acoustic wave controlling $[81,82]$. The key principle of transformation methods is based on the forminvariance of governing equations under an arbitrary transformation mapping. However, for elastic waves, Milton et al. showed that Navier's equations cannot maintain the forminvariance under a general spatial mapping [83], which means that methodologies applied in EM or acoustic transformations cannot be directly applied to control elastic waves. Specifically, by conducting the transformation of a governing equation in a polar coordinate system, Farhat et al. suggested a cylindrical cloaking for plate bending waves [84]. However, a systematical transformation method for controlling flexural waves has not been explicitly proposed based on an arbitrary transformation mapping. Recently, based on the assumption of local affine transformations, Hu et al. and Chang et al. proposed an approximate method to establish the transformation relations for elastic waves $[85,86]$. The method was numerically illustrated as a powerful tool to control elastic waves at highfrequencies or in small material-gradient elastic media. The method will serve as the theoretical transformation foundation both as a tool to provide flexural wave control on the general transformation mapping and determine the properties of the transformed materials. 
In this chapter, we theoretically and numerically demonstrate a design and numerical simulation of a flexural waveguide based on the active EMM. First, the transformation method for the flexural wave in the plate is developed and the transformed material properties are analytically derived. The physical realization of the transformed medium is proposed by an array of active EMMs in the form of discretization and truncation. The tunable effective properties are accomplished by integrating an electrorheological (ER) elastomer subjected to adjustable electric fields in the microstructure of the local resonator. Finally, the numerical simulation is conducted to demonstrate tunability and flexibility of the proposed waveguide over a broad frequency range and various steering directions without altering the microstructures. The study could have a very profound application in extraordinary wave beam-steering and structural health monitoring.

\subsection{A transformation method of controlling flexural waves in plates}

In general, the governing equation of flexural waves in an inhomogeneous isotropic plate can be expressed in the Cartesian coordinate system as [46]

$$
\nabla^{2}\left(D \nabla^{2} w\right)-(1-v)\left(\frac{\partial^{2} D}{\partial y^{2}} \frac{\partial^{2} w}{\partial x^{2}}-2 \frac{\partial^{2} D}{\partial x \partial y} \frac{\partial^{2} w}{\partial x \partial y}+\frac{\partial^{2} D}{\partial x^{2}} \frac{\partial^{2} w}{\partial y^{2}}\right)+\rho h \frac{\partial^{2} w}{\partial t^{2}}=0
$$

where $\nabla^{2}=\frac{\partial^{2}}{\partial x^{2}}+\frac{\partial^{2}}{\partial y^{2}}, w(x, y), \rho(x, y), h(x, y)$ and $v$ are the out-of-plane displacement field of the neutral plane, mass density, thickness and Poisson's ratio of the plate (which is usually assumed to be an constant), respectively, and the bending stiffness is defined as $D(x, y)=\frac{E(x, y) h^{3}(x, y)}{12\left(1-v^{2}\right)}$ with $E(x, y)$ being the Young's modulus. The transformation method can be applied to a general plate, however in this study, Kirchhoff plate theory is 
adopted for the interested frequency range because the wave length is much larger than the thickness of the plate, where the thickness shear deformation effect can be ignored. For $D(x, y)$ being a constant $D_{0}$, the governing equation can be reduced as

$$
D_{0} \nabla^{2}\left(\nabla^{2} w\right)+\rho h \frac{\partial^{2} w}{\partial t^{2}}=0
$$

where the phase velocity is defined as $c_{p}=\left(D_{0} \omega^{2} / \rho h\right)^{1 / 4}$.

In the study, the transformation method based on the assumption of a general mapping as a series of local affine deformations will be adopted and extended for controlling bending wave propagation in a plate. The basic idea of the method will be briefly reiterated for a general transformation mapping $\mathbf{x}^{\prime}=\mathbf{x}^{\prime}(\mathbf{x})$ from a virtual 2D space $\Omega$ to a physical 2D space $\Omega^{\prime}$, as shown in Fig. 6-1. In the virtual space $\Omega$, a local Cartesian frame $\mathbf{e}_{i}$ is established at a point $\mathbf{x}=(x, y)^{\mathrm{T}}$ and another local Cartesian frame $\mathbf{e}_{i}^{\prime}$ is formed at a point $\mathbf{x}^{\prime}=\left(x^{\prime}, y^{\prime}\right)^{\mathrm{T}}$ in the physical space $\Omega^{\prime}$. Under the affine deformation, the deformation gradient tensor of the mapping $\mathbf{A}=\nabla_{\mathbf{x}} \mathbf{x}^{\prime}$ can be decomposed into a rigid rotation tensor $\mathbf{R}$ and a pure stretch tensor $\mathbf{V}(\mathbf{A}=\mathbf{V R})$, where $\mathbf{V}=\lambda_{1} \mathbf{e}_{1}^{\prime} \otimes \mathbf{e}_{1}^{\prime}+\lambda_{2} \mathbf{e}_{2}^{\prime} \otimes \mathbf{e}_{2}^{\prime}$ with $\lambda_{1}$ and $\lambda_{2}$ being the principal stretches. Similarly, any physical and material quantities in the system should follow the decomposition of the transformation as

$$
\mathbf{V}_{\mathbf{q}} \mathbf{R}: q \rightarrow \mathbf{q}^{\prime}, q=w, E, \rho, h, v
$$

where $\mathbf{V}_{\mathrm{q}}$ is a scaling tensor of quantity $q$. Eq. (6.3) implies that their transformed quantities can be derived under the same rotation but different stretch in its local space element. The 
constraints on the form-invariance of the transformed governing equation as well as the energy conservation conditions will provide a way to determine the stretch tensor $\mathbf{V}_{\mathrm{q}}$.

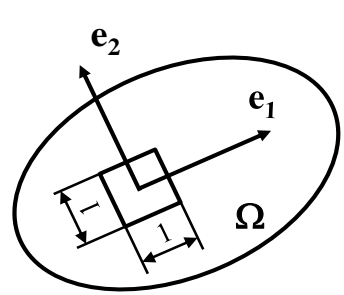

Virtual space

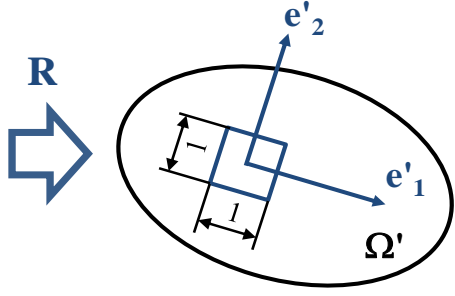

Physical space

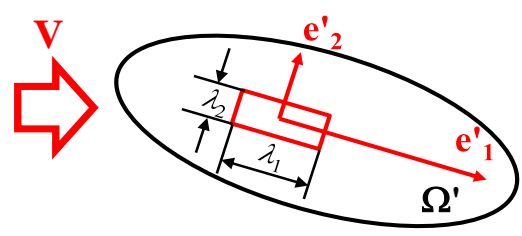

Physical space

Figure 6-1 Transformation mapping interpreted by local affine deformations

For the purpose of physical realization, a conformal transformation mapping is considered, which indicates that the principle stretches satisfy $\lambda_{1}=\lambda_{2}=\lambda$ and the resultant materials are isotropic. Under the conformal mapping, the transformed governing equation can be written locally with respect to the local Cartesian system $\mathbf{e}_{i}^{\prime}$ as

$\nabla^{\prime 2}\left(D^{\prime} \nabla^{\prime 2} w^{\prime}\right)-\left(1-v^{\prime}\right)\left(\frac{\partial^{2} D^{\prime}}{\partial y^{\prime 2}} \frac{\partial^{2} w^{\prime}}{\partial x^{\prime 2}}-2 \frac{\partial^{2} D^{\prime}}{\partial x^{\prime} \partial y^{\prime}} \frac{\partial^{2} w^{\prime}}{\partial x^{\prime} \partial y^{\prime}}+\frac{\partial^{2} D^{\prime}}{\partial x^{\prime 2}} \frac{\partial^{2} w^{\prime}}{\partial y^{\prime 2}}\right)+\rho^{\prime} h^{\prime} \frac{\partial^{2} w^{\prime}}{\partial t^{2}}=0$

where $\nabla^{\prime 2}=\frac{\partial^{2}}{\partial x^{\prime 2}}+\frac{\partial^{2}}{\partial y^{\prime 2}}, D^{\prime}\left(x^{\prime}, y^{\prime}\right)=\frac{E^{\prime}\left(x^{\prime}, y^{\prime}\right) h^{\prime 3}\left(x^{\prime}, y^{\prime}\right)}{12\left(1-v^{\prime 2}\right)}$ and $w^{\prime}, \rho^{\prime}, h^{\prime}, E^{\prime}$ and $v^{\prime}$ are corresponding out-of-plane displacement field, mass density, thickness, Young's modulus and Poisson's ratio of the plate in the transformed physical space $\Omega^{\prime}$, respectively. For the flexural wave, the transformations of the displacement, thickness and material parameters in the local Cartesian frame $\mathbf{e}_{i}^{\prime}$ according to Eq. (6.3) can be assumed as

$w^{\prime}=a w$

$\rho^{\prime}=b \rho$ 
$h^{\prime}=c h$

$E^{\prime}=d E$

$v^{\prime}=e v$

where the scaling factors $a, b, c, d$ and $e$ are unknown parameters to be determined.

Based on differential relations between two spaces $\partial / \partial x^{\prime}=(1 / \lambda) \partial / \partial x$ and $\partial / \partial y^{\prime}=(1 / \lambda) \partial / \partial y$, Eq. (6.4) can be rewritten in the initial space $\Omega$ as

$$
\begin{array}{r}
\frac{a c^{3} d}{\lambda^{4}}\left(\frac{\partial^{2}}{\partial x^{2}}+\frac{\partial^{2}}{\partial y^{2}}\right)\left[D\left(\frac{\partial^{2} w}{\partial x^{2}}+\frac{\partial^{2} w}{\partial y^{2}}\right)\right]-(1-e v) \frac{a c^{3} d}{\lambda^{4}}\left(\frac{\partial^{2} D}{\partial y^{2}} \frac{\partial^{2} w}{\partial x^{2}}-2 \frac{\partial^{2} D}{\partial x \partial y} \frac{\partial^{2} w}{\partial x \partial y}+\frac{\partial^{2} D}{\partial x^{2}} \frac{\partial^{2} w}{\partial y^{2}}\right) \\
+a b c \rho h \frac{\partial^{2} w}{\partial t^{2}}=0
\end{array}
$$

Comparing Eq. (6.6) to Eq. (6.1) by the form-invariance, the relation of the scaling factors is determined as

$\frac{d c^{2}}{b}=\lambda^{4}$

$e=1$

Thus, the scaled Poisson's ratio remains the original value during mapping to ensure the form-invariance of the governing equation for an inhomogeneous plate.

For the $2 \mathrm{D}$ transformation, it is natural to assume the thickness of the plate to be unchanged as $c=1$. Therefore, we have $d / b=\lambda^{4}$, which can be also expressed as 


$$
\frac{E^{\prime}}{\rho^{\prime}}=\lambda^{4} \frac{E}{\rho}
$$

On the other hand, potential and kinetic energy conservations need to be satisfied during the mapping. In the virtual space, potential and kinetic energies in a Kirchhoff plate can be expressed as

$$
\begin{aligned}
& P E=\frac{1}{2} D\left\{\left(\nabla^{2} w\right)^{2}-2(1-v)\left[\frac{\partial^{2} w}{\partial x^{2}} \frac{\partial^{2} w}{\partial y^{2}}-\left(\frac{\partial^{2} w}{\partial x \partial y}\right)^{2}\right]\right\} \mathrm{d} x \mathrm{~d} y \\
& K E=\frac{1}{2} \rho h\left(\frac{\partial w}{\partial t}\right)^{2} \mathrm{~d} x \mathrm{~d} y
\end{aligned}
$$

In the physical space, potential and kinetic energies of the plate are

$$
\begin{aligned}
& P E^{\prime}=\frac{1}{2} D^{\prime}\left\{\left(\nabla^{\prime 2} w^{\prime}\right)^{2}-2\left(1-v^{\prime}\right)\left[\frac{\partial^{2} w^{\prime}}{\partial x^{\prime 2}} \frac{\partial^{2} w^{\prime}}{\partial y^{\prime 2}}-\left(\frac{\partial^{2} w^{\prime}}{\partial x^{\prime} \partial y^{\prime}}\right)^{2}\right]\right\} \mathrm{d} x^{\prime} \mathrm{d} y^{\prime} \\
& K E^{\prime}=\frac{1}{2} \rho^{\prime} h^{\prime}\left(\frac{\partial w^{\prime}}{\partial t}\right)^{2} \mathrm{~d} x^{\prime} \mathrm{d} y^{\prime}
\end{aligned}
$$

By combing scaling factors in Eqs. (6.5a) - (6.5e) and the conformal transformation condition, Eqs. (6.10a) and (6.10b) can be rewritten in the initial space as

$$
\begin{aligned}
& P E^{\prime}=\frac{1}{2} \frac{a^{2} c^{3} d}{\lambda^{2}} D\left\{\left(\nabla^{2} w\right)^{2}-2(1-e v)\left[\frac{\partial^{2} w}{\partial x^{2}} \frac{\partial^{2} w}{\partial y^{2}}-\left(\frac{\partial^{2} w}{\partial x \partial y}\right)^{2}\right]\right\} \mathrm{d} x \mathrm{~d} y \\
& K E^{\prime}=\frac{1}{2} a^{2} b c \lambda^{2} \rho h\left(\frac{\partial w}{\partial t}\right)^{2} \mathrm{~d} x \mathrm{~d} y
\end{aligned}
$$


Based on the energy conservation, the relation of the scaling factors and principle stretch can also be derived, which is in the same form of Eq. (6.7). The result further validates the accuracy of the transformation method. In addition, the scaling factor of the transformed displacement, $a$, can also be determined as

$$
a=c \sqrt[4]{b d}
$$

It should be mentioned that transformation relations of flexural waves in the plate are different for elastic waves in the isotropic bulk media, where the transformation relation is $E^{\prime} / \rho^{\prime}=\lambda^{2} E / \rho[85]$.

\subsection{Mapping design of a beam steering of flexural waves}

The proposed transformation method is then used for the design of a flexural waveguide to steer flexural waves propagating along desired directions. In order to demonstrate this, we consider a rectangular plate in the virtual space with width $b=\left(R_{2}-R_{1}\right)$ and length $a$ respectively, as shown in Fig. 6-2. To achieve the conformal mapping, a two-step transformation mapping will be conducted. In the first step, the plate is transformed into the arc shape with a polar angle $\beta$. It is easily shown that there is only rescaling in the $\theta$ direction during the transformation, and this stretch can be obtained by comparing the arc length of the bender $\beta r$ with its original length $a$.

Therefore, two principle stretches at each point are

$\lambda_{r}^{\mathrm{I}}=1$

$\lambda_{\theta}^{\mathrm{I}}=\frac{\beta r}{a}=\frac{r}{R_{0}}$ 
where the superscript I represents the first-step transformation and $r=R_{0}$ is the arc where the length is unchanged during the transformation as $a=\beta R_{0}$. According to Eq. (6.13), this transformation will result in an anisotropic medium. To achieve a conformal mapping, a second-step transformation will be implemented to further adjust stretches in the $r$ direction as

$\lambda_{r^{\prime}}^{\mathrm{II}}=\frac{\mathrm{d} r^{\prime}}{\mathrm{d} r}$

$\lambda_{\theta^{\prime}}^{\mathrm{II}}=\frac{r^{\prime}}{r}$

where II indicates the second-step transformation. Therefore, the total isotropic stretches in $\theta^{\prime}$ and $r^{\prime}$ directions can be written as

$\lambda=\lambda_{r}^{\mathrm{I}} \lambda_{r^{\prime}}^{\mathrm{II}}=\lambda_{\theta}^{\mathrm{I}} \lambda_{\theta^{\prime}}^{\mathrm{II}}$

By substituting Eqs. (6.14a) and (6.14b) into Eq. (6.15), we have

$$
\lambda=\frac{\mathrm{d} r^{\prime}}{\mathrm{d} r}=\frac{r^{\prime}}{R_{0}}
$$

and required material parameters of the flexural waveguide can be determined as

$$
\frac{E^{\prime}}{\rho^{\prime}}=\left(\frac{r^{\prime}}{R_{0}}\right)^{4} \frac{E}{\rho}
$$

From Eq. (6.17), it can be concluded that the flexural waveguide can be achieved by finding materials with extremely gradient modulus or mass density, which is difficult to 
achieve using conventional materials. With the boundary condition that the arc length is unchanged, i.e. $r^{\prime}=R_{0}$, the transformation function $f(r)$ can be determined as

$$
r^{\prime}=f(r)=R_{0} e^{\frac{r-R_{0}}{R_{0}}}
$$

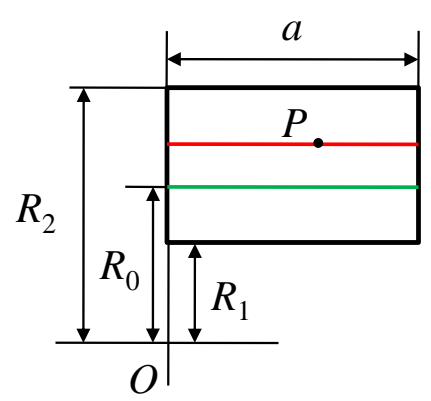

Virtual space
STEP. 1

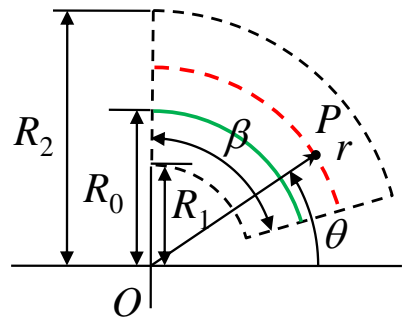

Physical space

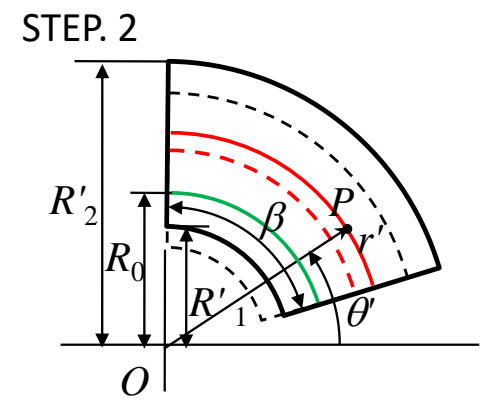

Physical space

Figure 6-2 Two-step transformation of an isotropic waveguide

\subsection{An active EMM for the flexural waveguide}

In this section, an active EMM is proposed for physical realization of a material with tunable effective mass density, and thus for actively steering of flexural waves over a broad frequency range. The unit cell of the active EMM is schematically shown in Fig. 6-3, where an active resonator is equipped with an ER elastomer annulus sandwiched between an aluminum annular ring and an aluminum cylinder. The resonator is surface bonded on the top of the host plate. In the figure, $a$ and $h$ are the side length and the thickness of the unit lattice of the aluminum host plate. The radius and the height of the aluminum cylinder are denoted as $r_{c}$ and $h_{c}$. The height and outer radii of the ER elastomer and aluminum annuluses are represented by $h_{a}, r_{e}$ and $r_{a}$, respectively. 
The working mechanism of the EMM can be briefly described as follows. When a bending wave propagates in the host plate with the surface-bonded EMM, the outer aluminum ring will be vibrated up and down with respect to the plate, and the ER elastomer will undergo shear-mode deformation. With the presence of the anti-resonant motion of the outer aluminum ring with respect to the host plate, the flexural wave energy is trapped in the resonator and an omnidirectional wave band gap will be observed, which can be interpreted by the negative effective mass density of the system. In additional, the tunability and flexibility of the active EMM can be accomplished by tuning the shear modulus of the ER elastomer annulus under timely adjustable electric fields along the radial direction without altering the microstructures.

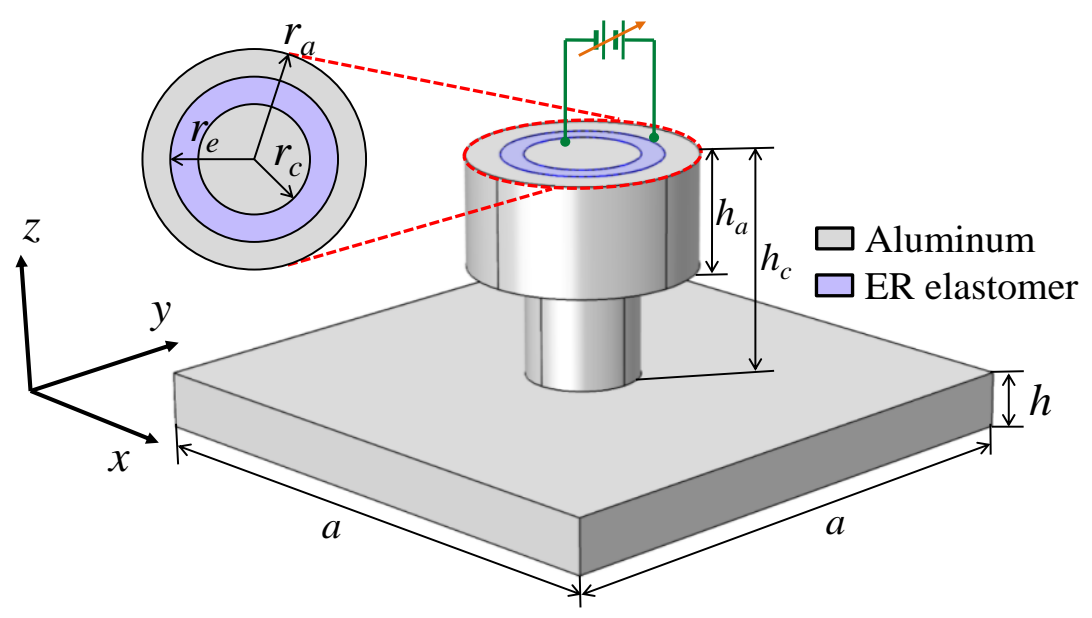

Figure 6-3 Unit cell of the active EMM surface-bonded on the plate

ER elastomers are soft elastomers by dispersing micrometer-size filler particles with high electrical permittivity and have adjustable rheological properties upon external electric loading, which induce stiffening or softening modulus of the elastomers. The 
relation between dynamic shear modulus of the ER elastomer, $G$, and applied electric field of magnitude, $\bar{E}$, can be approximately expressed as $[87,88]$

$G=G_{0}\left[1+\left(\bar{E} / E_{0}\right)^{2}\right]$

where $G_{0}$ is the shear modulus without electric field, and $E_{0}$ is a reference electric field. Based on the model presented by Wei et al. [89], effects of visoelastic properties of the ER are very small in the interested frequency range, and the imaginary portion of the shear modulus of the ER elastomer are not considered in the current study.

The numerically based effective medium method is used for determination of the outof-plane effective mass density of the EMM. By imposing the time-harmonic displacement constraints with $U_{x}=0, U_{y}=0$, and $U_{z}=A \mathrm{e}^{i \omega t}$ on the external surrounding boundaries, the effective mass density can be extracted as

$$
\rho_{\mathrm{eff}}=-\frac{F_{z}}{\omega^{2} A V}
$$

where $F_{\mathrm{z}}$ is the effective resultant force, and $V$ is the volume of the unit cell.

Figure 6-4 shows the normalized effective mass densities of the proposed EMM under various magnitudes of applied electric fields by the finite element software, COMSOL Multiphysics. In the figure, $\omega_{0}$ is the resonant frequency of the outer aluminum ring with $\bar{E}=0$, and the microstructure dimensions and constitute material properties of the EMM are listed in Table 6-1. 


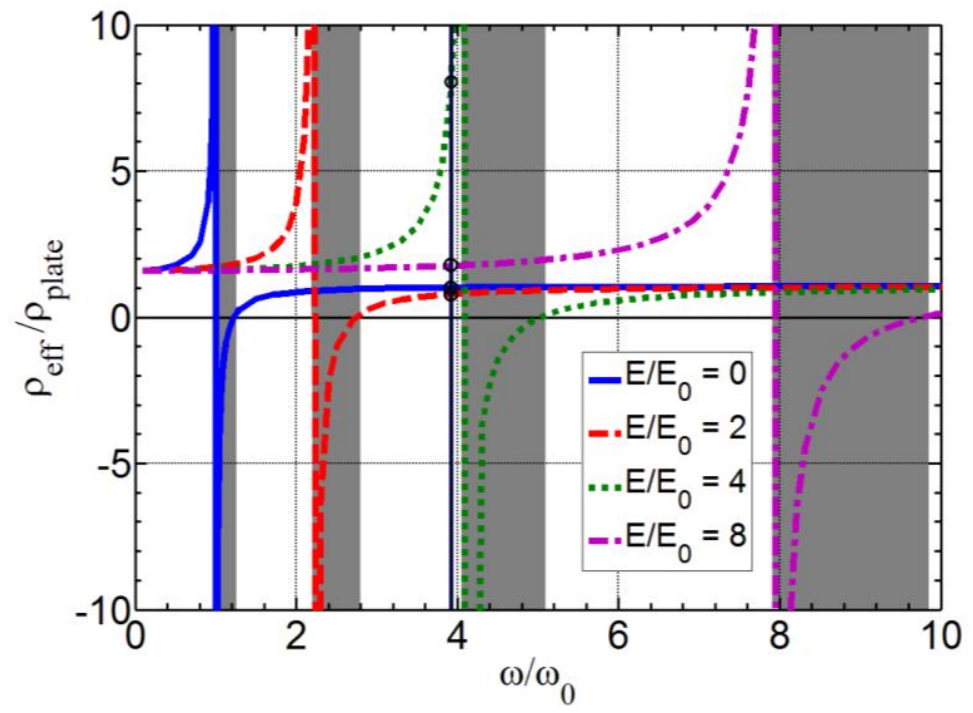

Figure 6-4 Effective mass density of the EMM under various magnitudes of electric fields

Table 6-1 Unit cell geometrical and material parameters of the active EMM

\begin{tabular}{cccc}
\hline \hline Geometrical properties (in mm) & \multicolumn{2}{c}{ Material properties } \\
\hline$a$ & 5 & Mass density (Aluminum) & $2700 \mathrm{~kg} / \mathrm{m}^{3}$ \\
$h$ & 1.5 & Lamés first parameter (Aluminum) & $51 \mathrm{GPa}$ \\
$r_{a}$ & 2 & Shear modulus (Aluminum) & $26 \mathrm{GPa}$ \\
$r_{e}$ & 0.7 & Mass density (ER elastomer) & $1000 \mathrm{~kg} / \mathrm{m}^{3}$ \\
$r_{c}$ & 0.5 & Lamé's first parameter (ER elastomer) & $1532 \mathrm{KPa}$ \\
$h_{a}$ & 2 & $G_{0}$ & $64 \mathrm{KPa}$ \\
$h_{c}$ & 1.8 & $E_{0}$ & $1.3 \mathrm{kV} / \mathrm{mm}$ \\
\hline \hline
\end{tabular}


As shown in the figure, the effective mass density reaches positive infinity at the resonant frequency and immediately turns to the negative infinity, then gradually approaches to a positive constant value at higher frequencies. Negative effective mass densities (shaded areas) can be found near the resonant frequency, i.e. $\omega=\omega_{0} \sim 1.3 \omega_{0}$, when $\bar{E}=0$. It can also be observed that the frequency range of the negative effective mass density can be shifted by changing the magnitudes of applied electric fields. For example, when $\bar{E}=2 E_{0}, 4 E_{0}$ and $8 E_{0}$, the frequency range is shifted to $2.2 \omega_{0} \sim 2.8 \omega_{0}, 3.9 \omega_{0} \sim 5.1 \omega_{0}$ and $8.1 \omega_{0} \sim 9.8 \omega_{0}$, respectively. It is understandable because the shear modulus of the ER elastomer is increased with the increase of the magnitude of electric field according to Eq. (6.19), which will result in the shift of the resonant frequency. It is also noticed that the frequency range of the negative effective mass density increases with the increase of the magnitude of the electric field. Finally, an extremely gradient distribution of effective mass density of the EMM can be accomplished by applying various magnitudes of electric fields at different frequencies. For example, as shown in the figure at $\omega=3.9 \omega_{0}$, the normalized effective mass density can be varied as $1.008,0.7874,7.555$ and 1.758 , respectively, when the electric field is tuned to be $\bar{E}=0,2 E_{0}, 4 E_{0}$ and $8 E_{0}$, respectively, which provides a potential solution for wave steering devices. It should be mentioned that the tunability of the effective mass density through electric fields could be implemented very fast and in real time, which opens a new way for many practical applications of the active EMM.

As discussed in the previous section, for a conformal mapping, the transformed medium for the flexural waveguide should possess the in-plane isotropic properties. To investigate wave behavior of the proposed EMM, iso-frequency contours of the flexural wave under an electric field are shown in Fig. 6-5. The contours are plotted in the first 
Brillouin zone and in the normalized wavenumber space. In the figure, microstructure dimensions and constitute material properties of the EMM are the same as those listed in Table 6-1, and magnitude of the applied electric field is $\bar{E}=8 E_{0}$. The unit of the frequency is $\mathrm{kHz}$. For all the wave vectors $\mathbf{k}=\left(k_{x}, k_{y}\right)$ in the first Brillouin zone, we can numerically calculate a series of functions $\omega=f\left(k_{x}, k_{y}\right)$ by applying the Bloch-Floquet periodic boundary conditions to the unit cell, which are denoted as iso-frequency curves. The iso-frequency curves can provide rich information about the wave propagation characteristics of the considered domain for the EMM, which is different from the dispersion curves to illustrate the passing and stop bands along the appropriate wave vector path. The direction of wave propagation can be easily obtained by displaying these angles on polar plots. The group velocity of wave motion in a two-dimensional EMM structure is given as

$$
\begin{gathered}
c_{g x}=\frac{\partial \omega}{\partial k_{x}} \\
c_{g y}=\frac{\partial \omega}{\partial k_{y}}
\end{gathered}
$$

For 2D EMMs, the characteristics of the wave propagation may be visualized in either of the following two ways: the energy flow is along the line of the steepest ascent on the iso-frequency surface, or equivalently, normal to the $\omega=$ constant lines on a contour plot of the iso-frequency surface. In this figure, nearly perfect circular contours are found at all interested frequencies, which illustrate the isotropic property of the proposed EMM because the group velocity vectors are collinear to the directions of wave propagation varying between $0^{\circ}$ and $360^{\circ}$. Similar wave phenomena can also be observed for the EMM under different electric fields. 


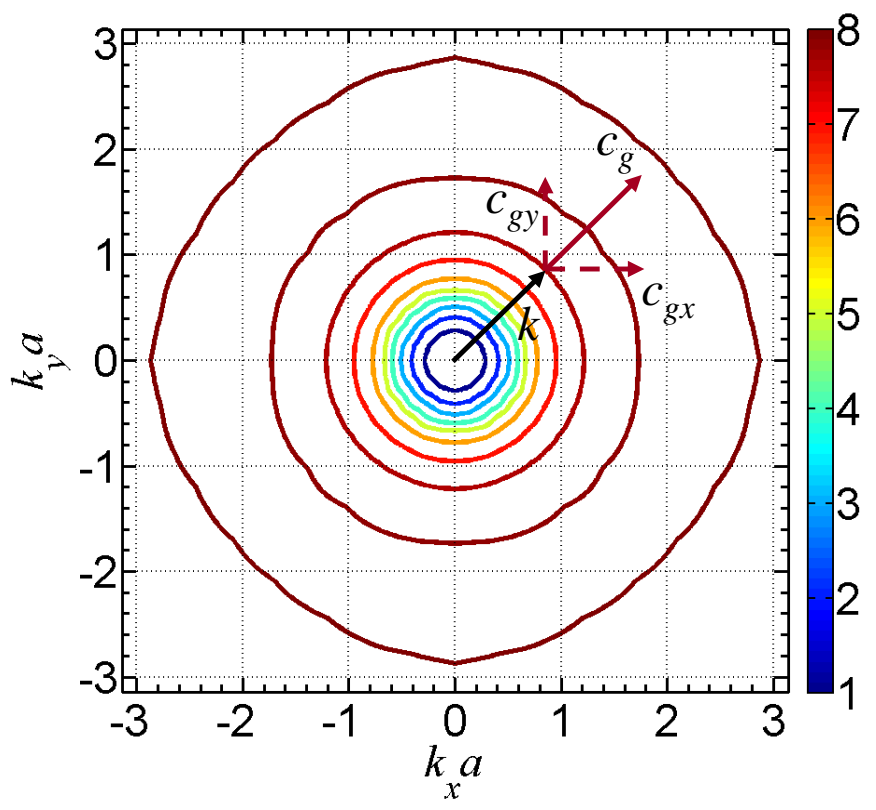

Figure 6-5 Iso-frequency contours of flexural waves of the active EMM

\subsection{Numerical simulations and discussions}

For the flexural waveguide application, since no homogeneous material exists that can reflect these properties in Eq. (6.17) based on the proposed mapping technique, we suggest a design to achieve such spatially varying properties using discrete distributions of active EMMs to perform the function. Control of the electric field adjusts the shear modulus of the ER elastomer which determines the dynamic density of the metamaterial. In this section, numerical simulations of the proposed flexural waveguide in a plate from the transformation method will be first conducted, in the meantime, the numerical simulation of the waveguide will be demonstrated by using an array of surface-bonded active EMMs. Specifically, tunability and flexibility of the waveguide are discussed for the extraordinary wave steering over a broad frequency range. 
Figure 6-6(a) shows the schematic model of the flexural waveguide with an arbitrary steering angle $\beta$ by the transformation method. According to Eq. (6.17), there exist many options for material property selection of the transformed medium. For the ease of the physical realization, the relationship of the transformed medium and the host plate is selected as

$E^{\prime}=E$

$\rho^{\prime}=\left(\frac{R_{0}}{r^{\prime}}\right)^{4} \rho$

As illustrated in the figure, an incident flexural wave beam is launched towards the designed waveguide, and the wave is then steered along the waveguide with a beam deflection angle $\beta$. Figure 6-6(b) shows the numerical simulation of the waveguide by using an array of the proposed active EMMs with a $90^{\circ}$ circular sector. The mass density profile of the waveguide from the transformation method will be approximately truncated by the discretely distributed EMMs under various electric fields. The shear modulus of the ER elastomer and applied electric fields are numerically determined through the wave dispersion analysis. For example, the wavenumber can be determined from the spatially varying densities (Eq. (6.17)) at a point as

$k_{b}(x, y)=\left(\frac{\rho(x, y) h \omega^{2}}{D}\right)^{\frac{1}{4}}$ 
from which the shear modulus or the magnitude of the applied electric field of the ER elastomer can then be iterated by matching the calculated eigenfrequency to the incident frequency.

For the array of EMMs with a $90^{\circ}$ sector, as shown in Fig. 6-6(b), the beam deflection angle $\beta\left(0^{\circ}<\beta<90^{\circ}\right)$ can be actively adjusted by properly selecting the controlled area of the EMM.

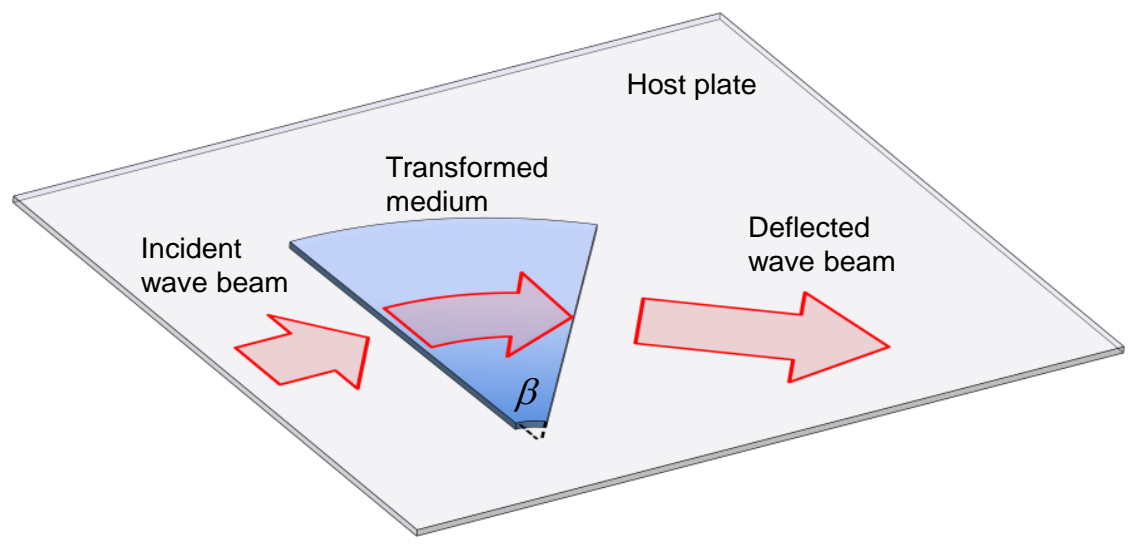

(a)

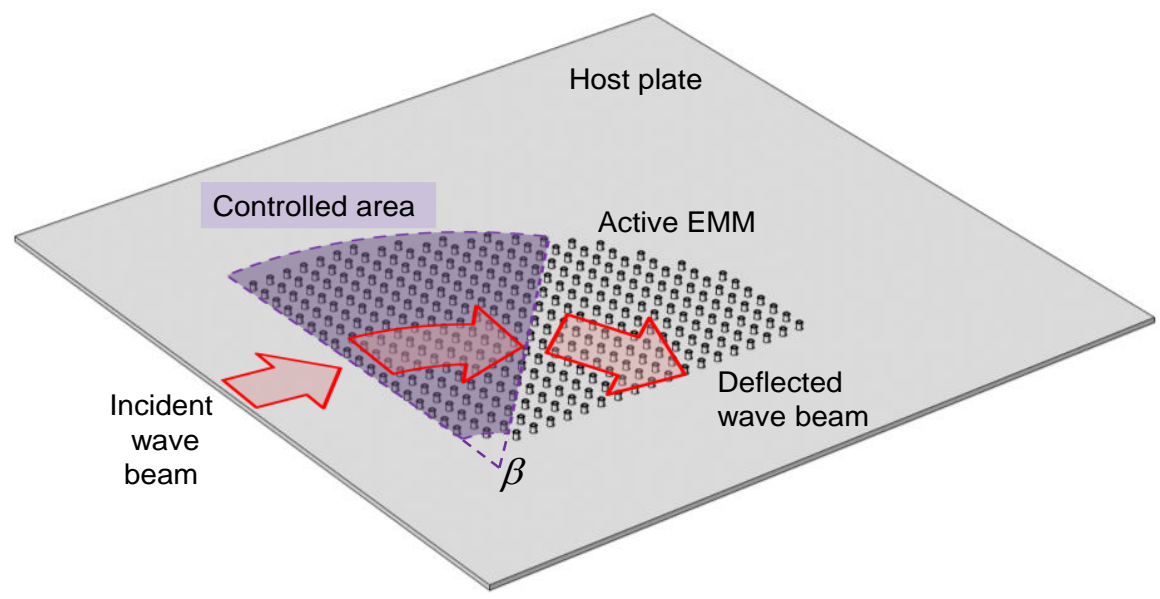

(b) 
Figure 6-6 (a) Schematic of a flexural waveguide with a deflection angle $\beta$ by the transformation method; (b) Numerical simulation of a flexural waveguide equipped with an array of active EMMs

The out-of-plane component of the displacement field associated with the flexural wave propagating in the plate with the designed waveguide is calculated. Figure 6-7 shows the out-of-plane wave field of the flexural waveguide with the deflection angle being $90^{\circ}$ based on the transformation method at the frequencies of $15 \mathrm{kHz}, 30 \mathrm{kHz}$ and $50 \mathrm{kHz}$, respectively. In the model, perfectly-matched-layers (PMLs) on the host plate are adopted to simulate infinite boundaries for flexural wave propagation. The material parameters of the host plate are the same as those listed in Table 1. The waveguide is designed with the inner and outer radii of the circular sector $\left(R_{1}^{\prime}\right.$ and $\left.R_{2}^{\prime}\right)$ being $10 \mathrm{~mm}$ and $100 \mathrm{~mm}$, respectively. The material properties of the transformed medium are selected according to Eq. (22). A harmonic antisymmetric Gaussian displacement profile is launched along the left edge of the plate. It can be observed that the direction of flexural waves can almost be deflected to $90^{\circ}$ for different frequencies. Small reflections are found on the interfaces between the waveguide and the background plate, due to the material mismatch between the host medium and transformed medium. In addition, scattered waves in the host plate leaking from the waveguide are also observed, which is caused by the width expansion of Gaussian beam. Specifically, when the incident frequency is increased from $15 \mathrm{kHz}$ to 50 $\mathrm{kHz}$, the scattered wave becomes nearly invisible. 


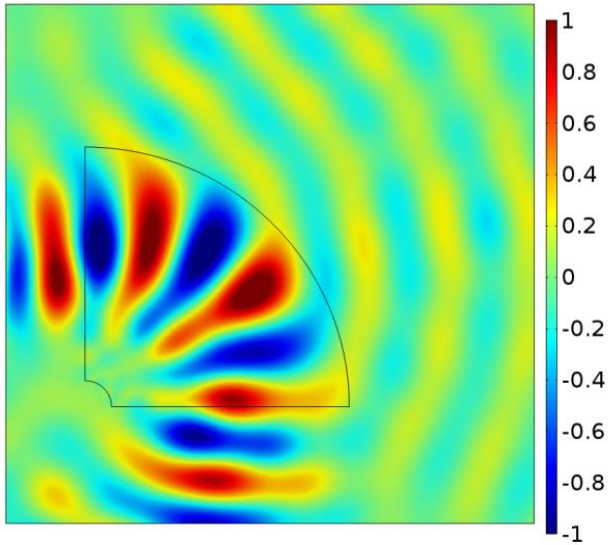

(a)

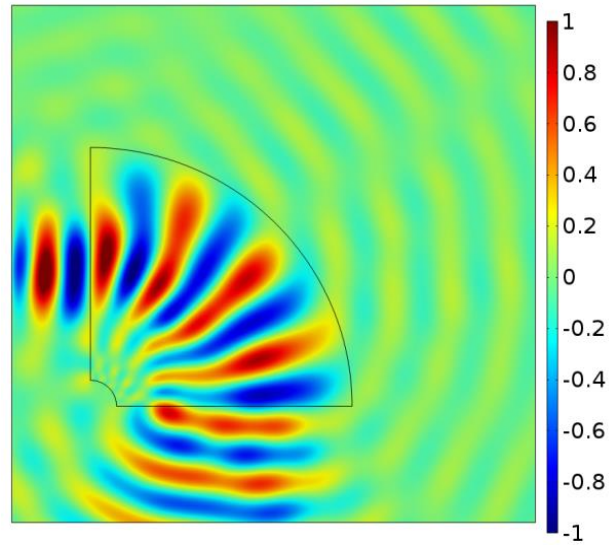

(b)

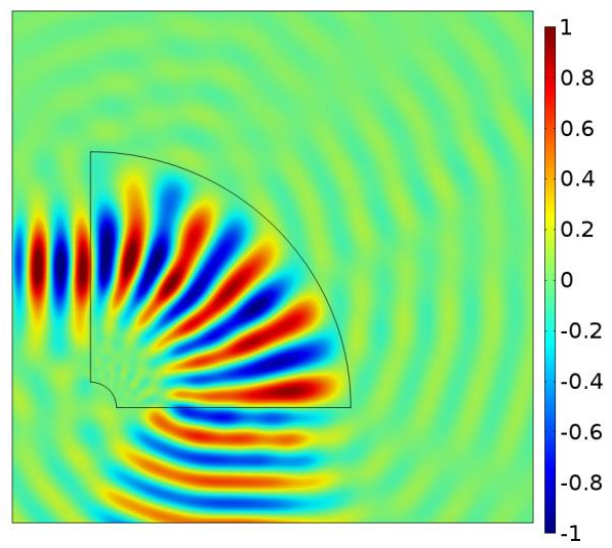

(c)

Figure 6-7 Normalized out-of-plane wave field of a $90^{\circ}$ flexural waveguide with material parameters from transformation method at different incident frequencies: (a) $15 \mathrm{kHz}$; (b) $30 \mathrm{kHz}$; (c) $50 \mathrm{kHz}$

For the physical realization of the waveguide, an array of active EMMs is proposed. Figure 6-8 shows the numerical demonstration of the flexural waveguide by using the active EMM at different operating frequencies of $15 \mathrm{kHz}, 30 \mathrm{kHz}$ and $50 \mathrm{kHz}$, respectively. For the active EMM, the outer radius of the aluminum ring $r_{a}$ is selected as $0.9 \mathrm{~mm}$, and 
other geometrical and material parameters are the same as those used in Figs. 6-4 and 6-5. In the simulation, the required mass density profiles at different frequencies are approximated by the discretely distributed EMM through actively adjusting the applied electric field on the ER elastomer without altering the microstructure. It can be found that flexural waves are indeed steered into $90^{\circ}$ as desired at all frequencies. Very good agreement with results predicted from the transformation method is observed, which shows the feasibility of the proposed active EMM. It is noticed that interior scattering waves within the discretely distributed EMM nearly vanish because of the unique subwavelength feature of the proposed EMM compared with the wavelength of the operating frequency in the host plate.

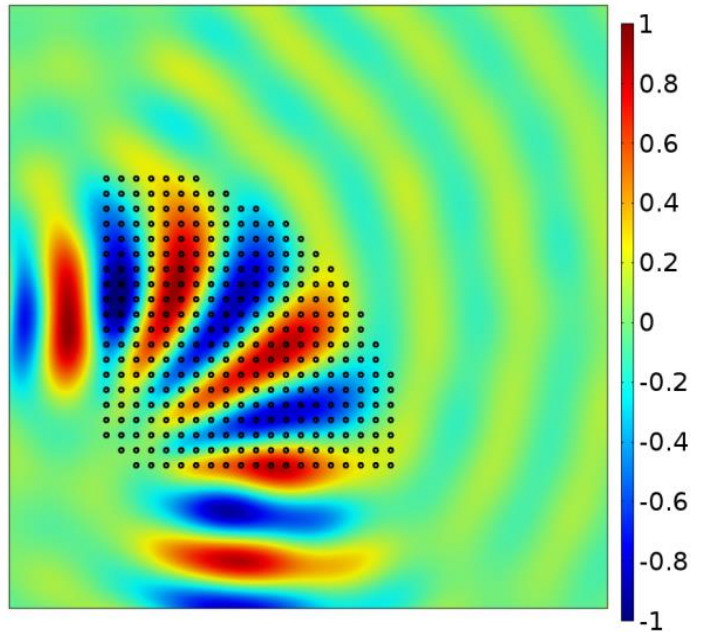

(a)

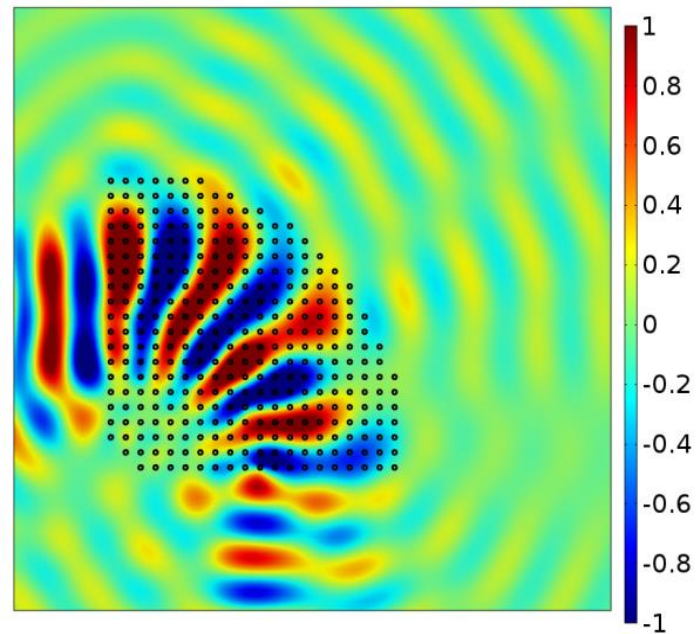

(b) 


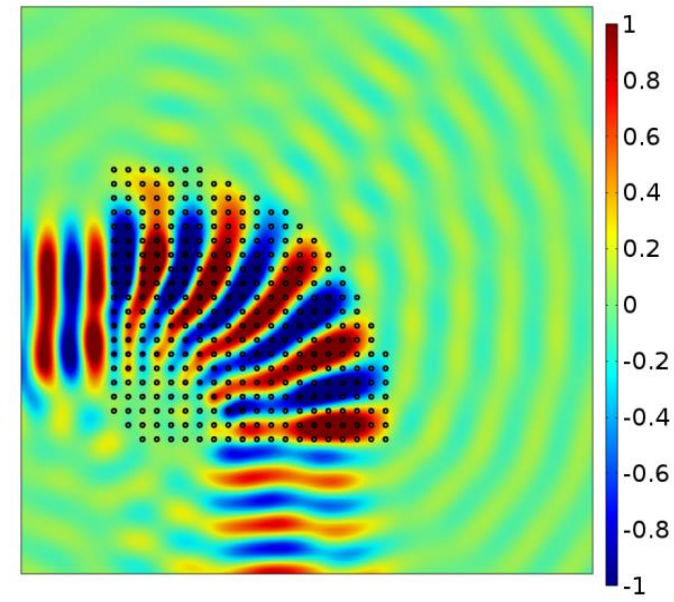

(c)

Figure 6-8 Normalized out-of-plane wave field of a $90^{\circ}$ flexural waveguide equipped with an array of active EMMs at different incident frequencies: (a) $15 \mathrm{kHz}$; (b) $30 \mathrm{kHz}$; (c) $50 \mathrm{kHz}$

Figure 6-9 shows the out-of-plane wave field of the flexural waveguide based on the proposed EMM at the operating frequency $15 \mathrm{kHz}$ with the deflection angle being 15, 45, $60^{\circ}$, respectively. In the simulation, material and geometrical parameters of the active EMM are the same as those used in Figure 6-8. As illustrated in the figure, the deflection angle can be flexibly tuned by applying electric fields on the selected areas (shaded areas) of the active EMM without altering the microstructures. The implied wave mechanism is that the effective mass density in the unselected area of the EMM is close to the mass density of the host plate at the operating frequency, therefore, effects on the flexural wave propagation due to the bonded EMM can be ignored. We anticipate the active EMM to be capable of steering arbitrary elastic beams to provide remarkable flexibility and open new 
exciting possibilities in a variety of elastic-based applications ranging from damage imaging to sensor device design.

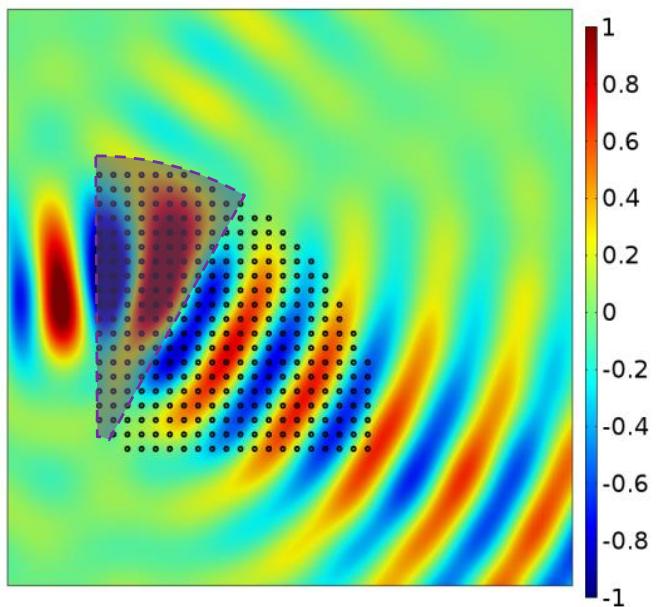

(a)

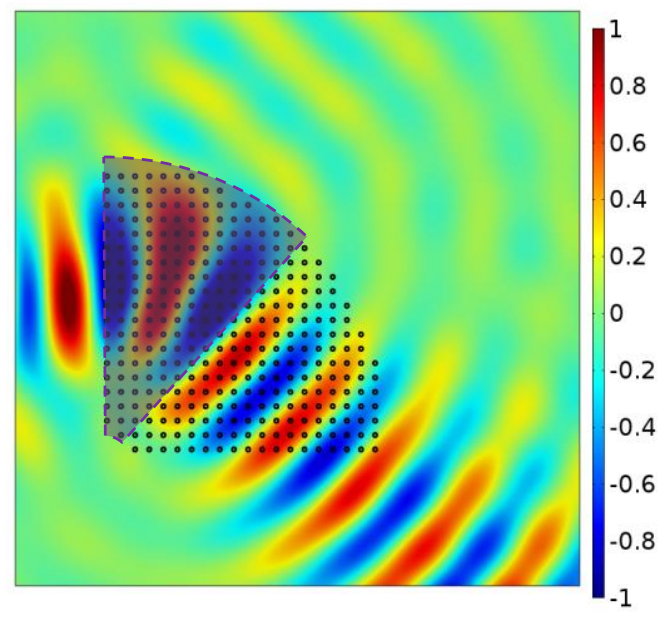

(b)

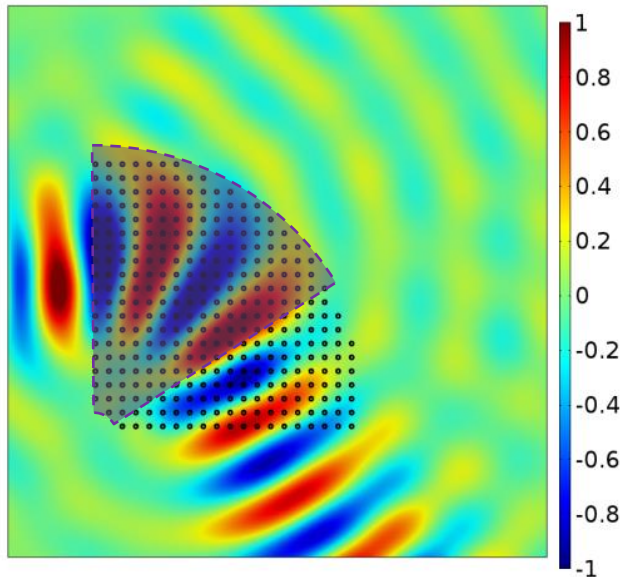

(c)

Figure 6-9 Normalized out-of-plane wave field of the flexural waveguide with controlled active EMMs at the operating frequency $15 \mathrm{kHz}$ with various deflection angles: (a) $15^{\circ}$;

(b) $45^{\circ}$; (c) $60^{\circ}$ 


\subsection{Summary}

In this chapter, we have designed the flexural waveguide that can fully control the flexural wave propagation in the plate. The transformation method for the flexural wave in the plate is first developed and the transformed material properties are analytically derived. The numerical simulation of the transformed medium is suggested by using the active EMM in the form of discretization and truncation. The tunable effective mass density is accomplished by applying an adjustable electric field in an ER elastomer under shear mode deformation. Finally, the numerical simulation is conducted to demonstrate tunability and flexibility of the proposed waveguide over a broad frequency range and various steering directions without altering the microstructures. The realization of the flexural waveguide opens new avenues for designing versatile compact acoustic components and have potential applications in a large variety of the fields such as damage detection and structural health monitoring. 


\section{Chapter 7 A Programmable Metasurface for Real Time Wavefront Control of Broadband Elastic Waves}

\subsection{Introduction}

In Chapters 5 and 6, we introduced active metamaterial-based waveguides with spatially varying material properties. However, there exist challenging scenarios where the capabilities of those approaches are not sufficient or extremely costly. These include cases where real-time and nonreciprocal asymmetric control of elastic/acoustic ray trajectories, be them linear or curved, blocked or amplified, is desired. Although a lot of efforts have been devoted to active elastic/acoustic metamaterials to serve those purposes, the resulting designs still tend to be bulky, invasive and necessitate impractical use of complex control systems.

Metasurfaces, a new kind of artificial planar metamaterials, have recently emerged as powerful means to transform incident waves by creating arbitrarily shaped transmitted or reflected wavefronts [90]. The transformation is accomplished by arranging suitable field discontinuities on a flat interface. Remarkably, due to dimensionality in 2D space for instance, the number of units constituting a metasurface scales approximately as the square root of that necessary in a bulk metamaterial. This significant reduction in the number of constitutive units makes metasurfaces excellent substitutes for bulk metamaterials offering thin designs with manageable control systems. The concept of metasurfaces has found a lot of interesting and unconventional applications in electromagnetic wave control [91-95]

and subsequently spread to acoustics [96-100] and elasticity [101-103]. However, passive 
metasurfaces with non-configurable functions can only operate in narrow frequency bands, which significantly limits their use in practice [95].

On the other hand, active metasurfaces with reconfigurable functionalities have received considerable interests recently in optics and acoustics and became a rapidly growing field in material science and engineering [95,100,104-107]. For example, by introducing voltage-controlled varactors $[95,104]$ or diodes [105] into resonating metaatom structures of electromagnetic metasurfaces, the effective index can be controlled locally along the metasurface to achieve desired the phase abrupt. A nonlinear reconfigurable metasurface was also proposed by blocking the incident acoustic wave and, at the same time, emitting a higher order harmonic transformed wave field [100,107]. However, extensions of active acoustic metasurfaces to the control of elastic waves in solids are not straightforward and none of successful designs on active elastic metasurfaces are reported so far.

Motivated by these considerations, this chapter introduces a simple platform of a programmable elastic metasurface [see Fig. 7-1(a)] and experimentally demonstrates, for the first time, real-time reconfigurable and multifunctional control of flexural waves in plates. The proposed metasurface consists of a 1D array of self-sensing-and-actuating units composed of piezoelectric patches bonded on a plate, each unit being controlled by a simple digital circuit, which can be remotely coded through wireless communications. In the adopted paradigm, the local wave transmittance and phase profiles that encode the current functionality of the metasurface can be repeatedly tuned in real time with the programmed circuits to achieve different functionalities with quasi-instantaneous response times compared to the time period of the propagating waves. In this manner, real-time wave 
steering and curvilinear trajectory adjustment are demonstrated experimentally. The functionalities that can be programmed into the metasurface are by no means limited to single ray path control and are extended to simultaneous multifunctional wave control. To further exemplify the range of functionalities that can be attained, an experimental demonstration of one-way non-reciprocal wave transmission is presented. In this configuration, the metasurface blocks waves incident in a given direction while amplifying

waves incident in the opposite direction. The application of the metasurface to skin cloaking is illustrated numerically as well. Last, operability in broadband frequency ranges is investigated. The proposed concept of a programmable elastic metasurface should significantly enhance elastic wave control techniques in a way that will benefit the fields of structural health monitoring and ultrasonic imaging especially in complex heterogenous structures, not to mention active and unidirectional noise cancellation and stealth technologies.

\subsection{Design principles}

To construct the metasurface, a 1D periodic array of thin slits is delicately cut into a host steel plate [see Fig. 7-1(a)]. Sensing and actuating beams are then formed between the slits. Each one of the sensing beams is bonded with two identical piezoelectric patches placed symmetrically with respect to the metasurface whereas each actuating beam is bonded with one piezoelectric patch located at the mid-thickness of the metasurface. Sensing beams will extract the background incident wave signal based on which, through a "feedforward" control loop, the actuating beams will generate coherent waves thus allowing, by constructive and/or destructive interference, to control both the phase and amplitude of the transmitted signal. 


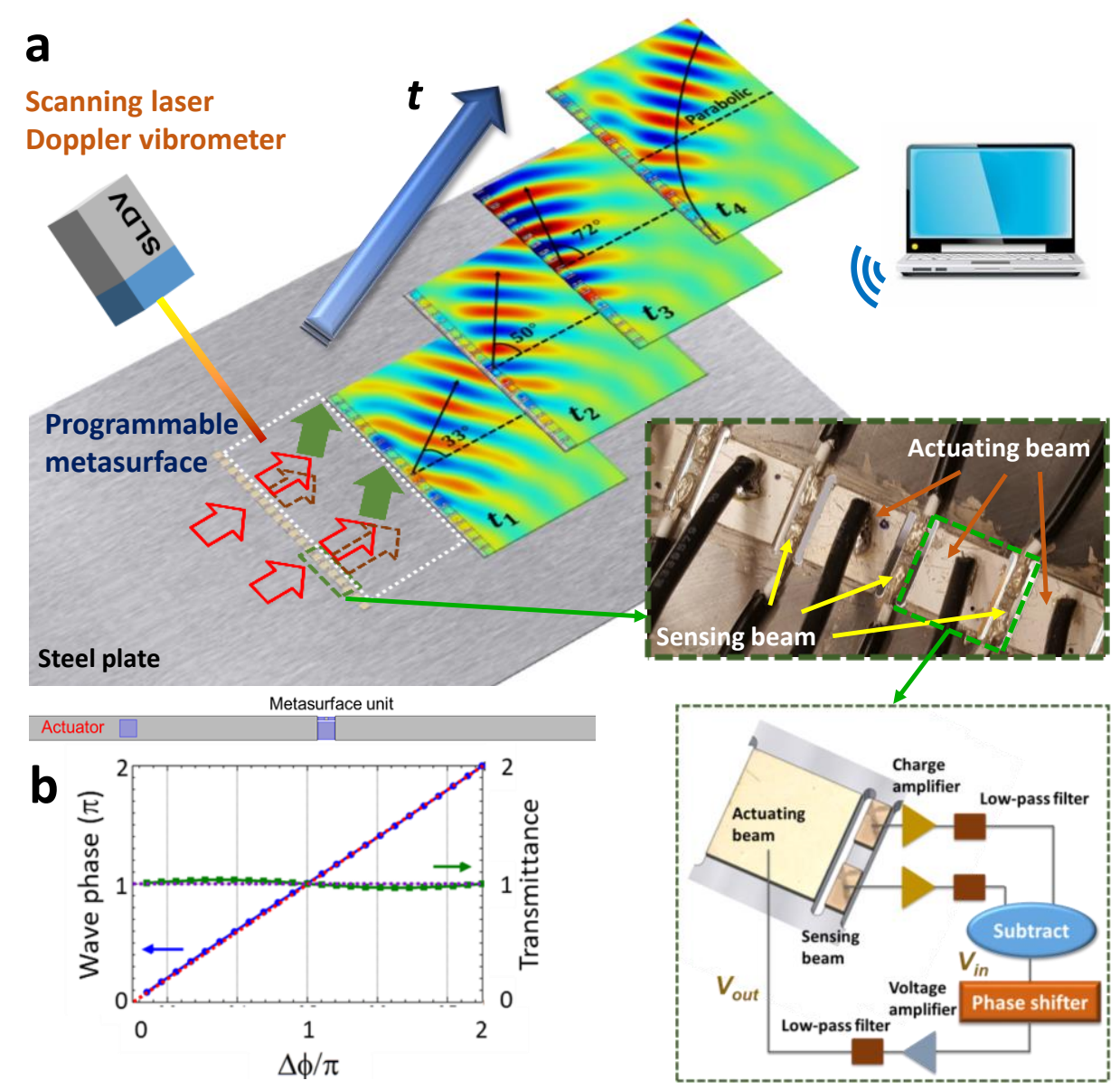

Figure 7-1 Schematic of the programmable metasurface and its wave transmittance and phase jump characteristics. (a) Illustration of the experimental setup and real time elastic ray control. Red, brown and green solid arrows represent incident, coherent and superposed total wave fields, respectively. By programing the digital circuits of the metasurface, wavefronts are shaped and tuned in real time. The transformed wavefronts are finally measured by a scanning laser Doppler vibrometer just behind the metasurface. (b) Wave transmittance and induced phase jump determined analytically and numerically for a single unit cell in a quasi-1D setting.

Sensors and actuators communicate through a digital-circuit control system [see Fig. 7-1(a)]. In each unit cell, the two sensors are individually connected to two charge 
amplifiers and two low-pass band filters. The filtered voltage signals coming from the sensors contain contributions from both the incident wave and the coherent waves generated by the actuators. Subtracting the output signals of each pair of sensors yields an input signal, called $V_{i n}$, where, due to mirror symmetry, the contribution of generated coherent waves has been eliminated and only the contribution of the background incident signal is retained. It should be mentioned that the subtraction operation not only removes intra-cell actuator-to-sensor feedback effects but further eliminates inter-cell effects as well. This decoupling ensures that the metasurface functionality can be programmed locally unit cell by unit cell and thus greatly reduces the complexity of the underlying electrical control system. Note that in general, scattering of waves by the piezoelectric patches at subwavelength scales is weak and will be ignored.

In this study, the Kirchhoff plate theory is adopted for the interested frequency range of the metasurface, because the wavelength is much larger than the thickness of the plate $(\lambda / h \approx 18$ at $10 \mathrm{kHz})$, where the thickness shear deformation effect can be ignored. The governing equation of flexural waves in a homogeneous isotropic plate can be expressed as

$$
D \nabla^{2}\left(\nabla^{2} w\right)+\rho h \frac{\partial^{2} w}{\partial t^{2}}=0
$$

where $\rho$ and $h$ represent the mass density and thickness of the plate, and $\nabla^{2}=\frac{\partial^{2}}{\partial x^{2}}+\frac{\partial^{2}}{\partial y^{2}}$, $D=\frac{E h^{3}}{12\left(1-v^{2}\right)}$ with $E$ and $v$ being the Young's modulus and Poisson's ratio of the host plate material. 
For one-dimensional harmonic wave analysis, we select one metasurface unit cell with periodic boundary conditions as the schematic shown in Fig. 7-2. The incident plane wave is assumed to propagate along the positive $x$ direction as (a wave solution to Eq. (7.1))

$$
w_{i}=w_{0} e^{i k x} \text {, for } x \leq-l_{m} \text { and } x \geq l_{m}
$$

where the wavenumber $k=\left(\frac{\rho h \omega^{2}}{D}\right)^{\frac{1}{4}}, w_{0}$ represents the complex wave amplitude, and the reflections due to the presences of the slits and surface-bonded piezoelectric plates are ignored because of their subwavelength dimensions and small impedance mismatch. We also assume that the incident wave is with continuous phase when travelling through the sensing and actuating beams, which is still due to the subwavelength feature of the thin metasurface.

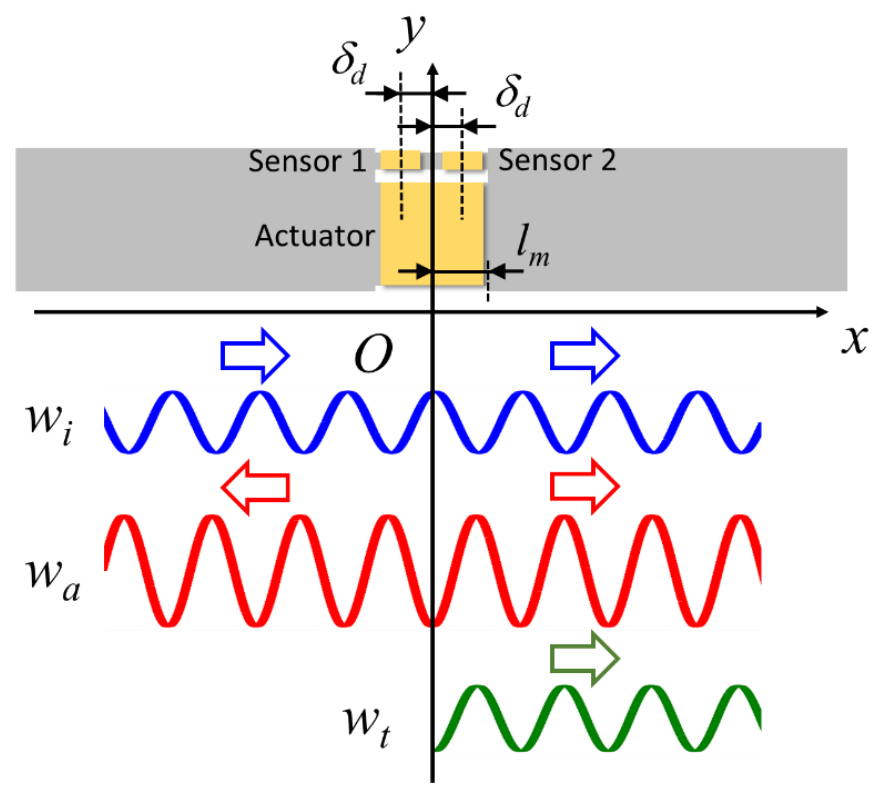

Figure 7-2 Schematic of the one-dimensional harmonic wave analysis of the transmission-type metasurface

The displacement incident wave field in the sensing beam is however assumed as 
$w_{i}^{(s)}=w_{0} e^{i k_{b} x}$

where the wavenumber $k_{b}=\left(\frac{\bar{\rho} \omega^{2}}{D_{b}}\right)^{\frac{1}{4}}$ with $D_{b}$ and $\bar{\rho}$ being the effective bending stiffness and mass density per unit length of the sensing beam with piezoelectric sensors attached. In Eq. (7.3), the wave amplitude is assumed as the same with that of the incident wave in the host plate. Based on our numerical simulations, all those assumptions made above are very reasonable and accurate for the wave propagation analysis.

The voltage signal acquired from the charge amplifier connected with "Sensor 1" can be expressed as

$V_{1}=\frac{Q_{1}}{C_{0}}$

where the free charge on the electrode $Q_{1}=\int_{S_{1}}-e_{31} \varepsilon_{x} d S$, with $e_{31}$ being the piezoelectric constant and the strain being $\varepsilon_{x}=k_{b}^{2} \tilde{h}_{p} w_{0} e^{i k_{b} x}$ base on the Kirchhoff plate assumption, in which $\tilde{h}_{p}$ represents the distance between the upper surface of the piezoelectric plate to the neutral plane of the sensor beam. It should be mentioned that this signal only represents the incident wave component. The coherent wave components generated by metasurface actuators, which will be finally canceled out, is useless to be expressed at this point.

Alternatively, Eq. (7.4) can be rewritten as

$V_{1}=\kappa_{s} A w_{0} e^{-i k_{b} \delta_{d}}$ 
where $\kappa_{s}=\frac{-e_{31} k_{b}^{2} \tilde{h}_{p} \int_{S_{1}} e^{i k_{b} x} d S}{C_{p}^{T} e^{-i k_{b} \delta_{d}}}$ is defined as the electromechanical coupling coefficient of the piezoelectric sensor, and $A=\frac{C_{p}^{T}}{C_{0}}$ is the amplification ratio of the charge amplifier. Similarly, the voltage signal acquired from the charge amplifier of "Sensor 2" becomes $V_{2}=\kappa_{s} A w_{0} e^{i k_{b} \delta_{d}}$

According to the design principles illustrated in the main text of the article, the input signal, $V_{i n}$, attained from the two sensors is

$V_{i n}=V_{1}-V_{2}=-i \kappa_{s} A w_{0} \sin \left(k_{x} \delta_{d}\right)$

The output signal from the electrical control system, $V_{\text {out }}=H V_{\text {in }}$, is then applied across the corresponding actuator in the metasurface unit cell, which generates two coherent waves propagating to both sides of the metasurface. Specifically, the wave field in the right-hand-side of the metasurface, which is proportional to the voltage, $V_{\text {out }}$, can be expressed as

$w_{a}=i \kappa_{a} V_{\text {out }} e^{i k x}, x>l_{m}$

where we define $\kappa_{a}$ as the electromechanical coupling coefficient of the piezoelectric actuator. Finally, the total transmitted wave field is

$$
w_{t}=\left[1+\kappa_{s} \kappa_{a} A H \sin \left(k_{x} \delta_{d}\right)\right] w_{0} e^{i k x}
$$

It can be found from Eq. (7.9) that both the amplitude and the phase of the transmitted wave can be independently controlled by the transfer function. For transmission-type metasurfaces with only phase engineering, the transmittance is usually designed to be unitary, therefore, 


$$
H=\frac{e^{i \phi}-1}{\kappa_{s} \kappa_{a} A \sin \left(k_{x} \delta_{d}\right)}=C\left(e^{i \phi}-1\right)
$$

in which $\phi$ is the desired phase abrupt induced by the metasurface and the amplification ratio $C=1 /\left(\kappa_{s} \kappa_{a} A \sin \left(k_{x} \delta_{d}\right)\right)$. Due to the geometric complexity, the constant, $C$, is extremely difficult to be determined analytically. A numerical iteration approach is then applied. For example, we first prescribe the phase abrupt, $\phi$, by $\pi$. By selecting the constant, $C$, randomly, a resulting displacement wave field will be obtained. Finally, we compare the amplitude of the transmitted wave with that of the incident wave, and correct the old "C" by a new " $C$ ".

In Fig. 7-3, three-dimensional piezoelectric coupled numerical simulations with COMSOL Multiphysics are performed to validate the one-dimensional wave phase control ability of the programmable metasurface at $10 \mathrm{kHz}$. Geometric parameters are illustrated in Fig. 7-3(a) with the values listed in Table 7-1. The thickness of the host steel plate is selected as $3.0 \mathrm{~mm}$ and the thicknesses of sensor and actuator patches are $1.1 \mathrm{~mm}$ and 1.0 $\mathrm{mm}$, respectively. As shown on the top portion in Fig. 7-3(b), the top and bottom boundaries of the simulated domain are set to be symmetric. The out-of-plane displacement wave field in the figure illustrates that the phase change introduced into the transmitted wave is almost exact the same with the phase, $\phi$, prescribed in the simulation by using Eq. (7.10), where $\phi=0, \pi / 3,2 \pi / 3, \pi, 4 \pi / 3,5 \pi / 3$ and $2 \pi$, respectively. It can also be found from the figure that the amplitudes of transmitted waves among all the cases are almost identical. 

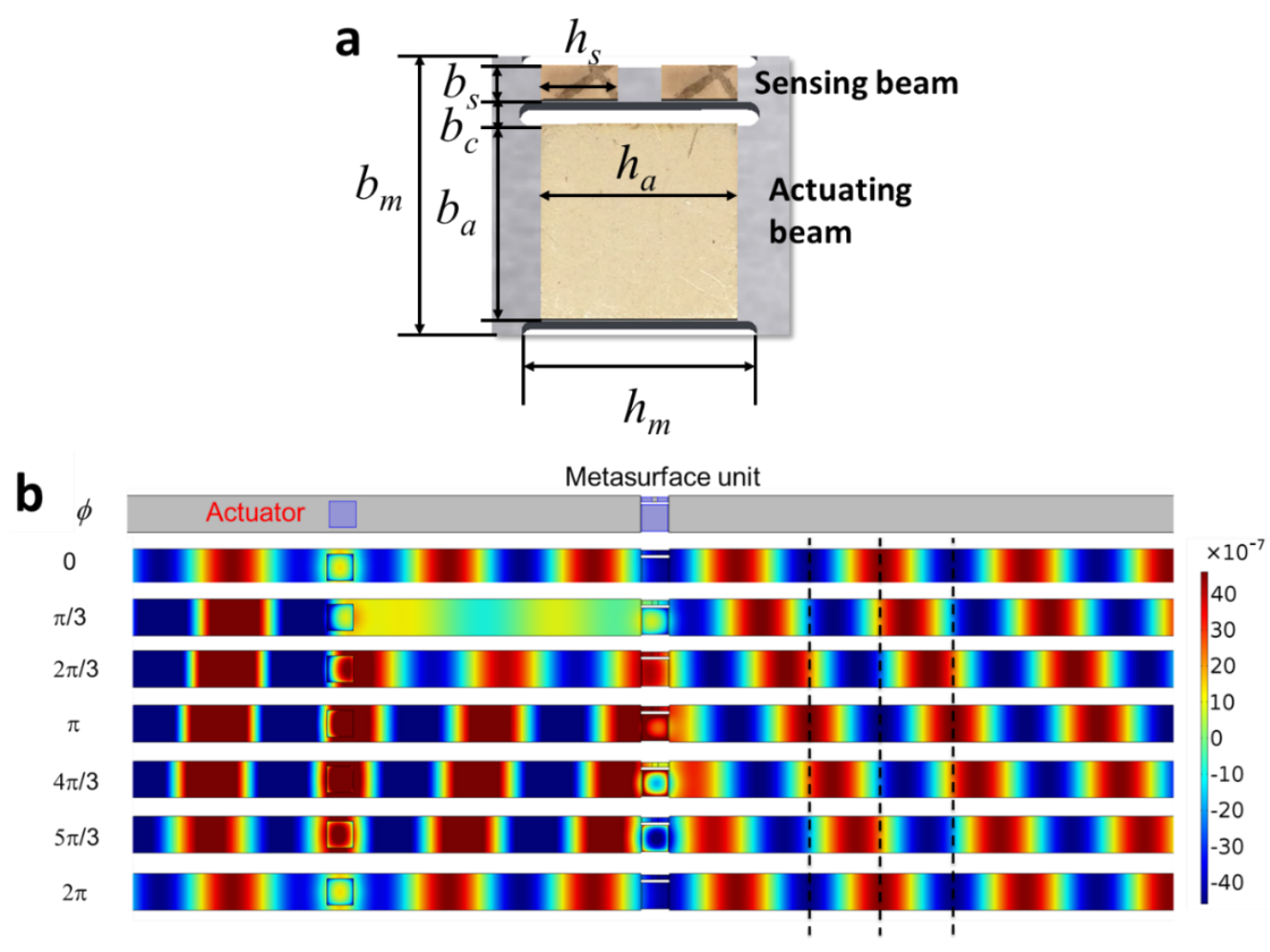

Figure 7-3 (a) Geometric parameters of the unit cell of the transmission-type metasurface;

(b) Out-of-plane displacement wave field manipulated by the metasurface with different phase abrupt in simulations

Table 7-1 Geometric parameters of the transmission-type metasurface

\begin{tabular}{llllllll}
\hline$b_{s}$ & $1.75 \mathrm{~mm}$ & $b_{m}$ & $14.0 \mathrm{~mm}$ & $h_{s}$ & $3.9 \mathrm{~mm}$ & $h_{m}$ & $11.0 \mathrm{~mm}$ \\
$b_{c}$ & $1.25 \mathrm{~mm}$ & $b_{a}$ & $10.0 \mathrm{~mm}$ & $h_{a}$ & $10.0 \mathrm{~mm}$
\end{tabular}

In order to achieve the transfer function of Eq. (7-10), phase shifter and voltage amplification circuits are constructed and are shown in Fig. 7-1(a). Note that a unitary transmittance does not mean that no extra elastic energy is radiated in the direction of the incident wave. In fact, the phase jump of the transmitted wave is purely caused by the 
elastic energy input from the electrical domain. For the transmission-type metasurface, only the transmitted half of that energy is useful whereas the backscattered half is lost.

Piezoelectric-coupled 3D numerical simulations were performed to validate the phase control abilities of a single unit cell at $10 \mathrm{kHz}$ [see Fig. 7-1(b)]. Therein, the top and bottom boundaries of the simulated domain are set to be symmetric and constant $C$ is determined numerically. On the figure, analytical predictions based on Eq. (7-10) (dashed lines) as well as simulated values (solid curves) of the phase and transmittance are plotted against one another and show satisfactory agreement. The results illustrate how full phase control of the transmitted wave from 0 to $2 \pi$ altogether while uniformly maintaining nearly unitary transmittance can be achieved simply by programming the electrical system within a unit cell. The small fluctuations in the simulated transmittance are caused by weak reflections due to the presence of sensors and actuators on the plate.

\subsection{Results and discussion}

\subsubsection{Real time steering of rays}

Just as the transfer function $H$ controls the effect of one unit cell, the profile $H=H(y)$ where $y$ is the coordinate along the metasurface encodes the function of the metasurface as a whole. For instance, the generalized Snell's law dictates that the "refraction" angle $\theta_{t}$ of the transmitted wave is related to the phase gradient $\frac{\Delta \phi}{\Delta y}$ according to $\sin \theta_{t}=\frac{\Delta \phi}{k \Delta y}=\frac{\lambda}{\Delta d}$ [91], where $y$ is the coordinate along the metasurface, $\Delta d$ is the distance along the metasurface across which the prescribed phase changes by $2 \pi$ and $\lambda=53.25 \mathrm{~mm}$ at $10 \mathrm{kHz}$. Accordingly, controlling the phase profile along the interface is synonymous to controlling 
the ray paths of the transmitted wave. In particular, a uniform $\Delta d$, equivalent to a linear phase profile, implies that the transmitted wave has uniform and rectilinear ray paths.

In the experimental demonstration, to fabricate the corresponding sensing and actuating beams, a periodic array of thin slits was cut by a fiber laser cutting machine on a steel plate with the dimensions being $1 \mathrm{~m} \times 2 \mathrm{~m} \times 3 \mathrm{~mm}$. An array of piezoelectric plates (APC 850, APC 855) was then bonded on the surfaces of those sensing and actuating beams by a conductive epoxy (Chemtronics) to comprise those sensors and actuators of a metasurface with 20 unit cells. Another array of piezoelectric plates (APC 850) was bonded in the lefthand-side $120 \mathrm{~mm}$ away from the metasurface to generate normally incident plane flexural waves. Each of the metasurface unit cells had their own individual circuit control system which was divided into several small parts with specifically assigned functions. All the circuits were fabricated on printed circuit boards, which were finally assembled into two large boards for the whole 20 unit cells. To produce an incident plane wave, a 10-peak tone burst signal with central frequency being $10 \mathrm{kHz}$ was generated by a Tektronix AFG3022C arbitrary waveform generator and amplified by a Krohn-Hite high voltage power amplifier, which was finally applied across the actuator array in the left-hand-side of the metasurface. The transmitted out-of-plane velocity wave field was measured by a Polytec OFV-055 scanning laser Doppler vibrometer (SLDV). To ensure circuits having stable and high signal-to-noise-ratio signals, the cutoff frequencies of the low-pass band filters were dedicatedly tuned in experiments.

During the first three time intervals [see Figs. 7-4(a) - 7-4(c)], a uniform $\Delta d$ is prescribed and changed from 98 to 70 and then to $56 \mathrm{~mm}$. By reprogramming the phase profile of the metasurface correspondingly, the steering angle of the transmitted wave is 
controlled and changed from $33^{\circ}$ to $50^{\circ}$ and then to $72^{\circ}$ in real time. The plots show that numerical and experimental results agree well and that steering angles coincide. Moreover, the possibility for programming nonlinear phase profiles offers the possibility to steer the transmitted ray paths according to curved trajectories. All other things being equal, in the fourth time interval, the phase profile along the metasurface is changed from a linear one to the one given by $\phi=\frac{\pi a^{2}}{2 \lambda} \ln \left[y+\sqrt{y^{2}+\left(\frac{a^{2}}{4}\right)^{2}}\right]$ with $a=18.67 \mathrm{~mm}$ in order to realize parabolic transmitted ray paths. In Fig. 7-4(d), numerical and experimental tests demonstrate the phenomenon. Such extreme examples of real-time control of elastic waves based on simple programmable digital circuits have never been demonstrated before and should offer new possibilities in the field of structural health monitoring and ultrasonic imaging especially in complex heterogenous structures.

a

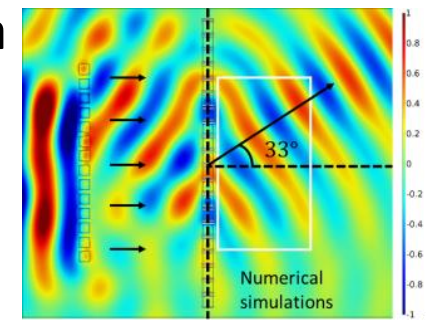

C

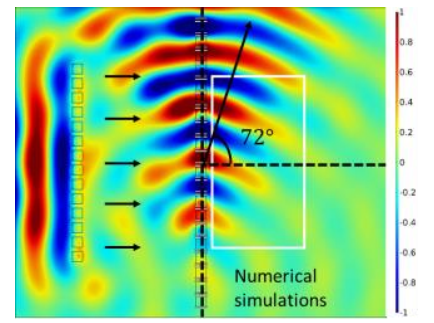

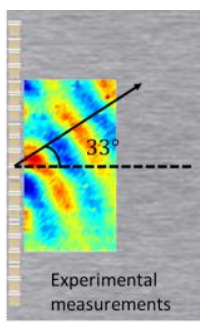

b
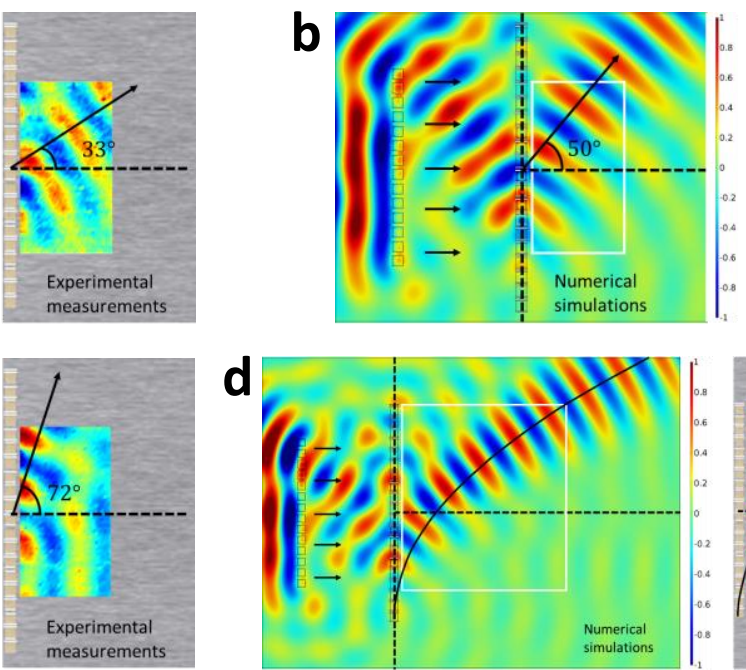

d

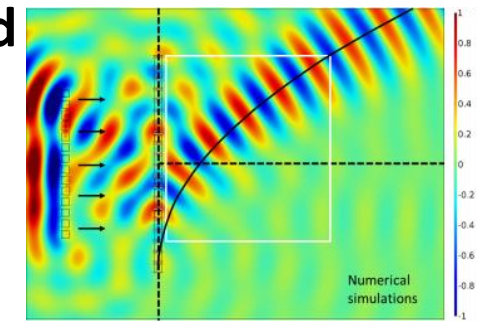

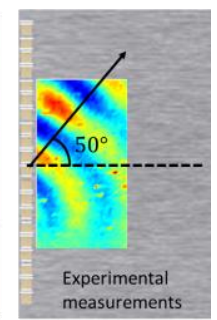

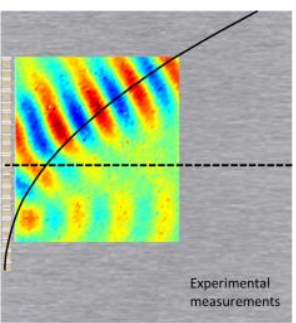

Figure 7-4 Real time steering of elastic rays. (a-d) Numerically simulated and experimentally measured out-of-plane velocity fields manipulated by the metasurface programmed with different phase profiles during different time intervals: (a) Steering elastic rays to $33^{\circ}$ during the first time interval; (b) Steering elastic rays to $50^{\circ}$ during the 
second time interval; (c) Steering elastic rays to $72^{\circ}$ during the third time interval; (d) Achieving a parabolic ray path during the fourth time interval. In all cases, the rightgoing plane incident wave is generated by an array of piezoelectric patches. On the numerical plots, the regions framed in white correspond to zones where experimental measurements were obtained. Black arrows and curve represent the desired elastic ray direction and trajectory determined analytically. Good agreements between analytical, numerical and experimental results are clearly seen at all times

\subsubsection{Multifunctional transfer functions}

It is worth mentioning that the functionalities of the programmable metasurface can be superposed by appropriately "summing" the transfer functions of each functionality. Given the phase profile necessary for steering $\phi^{(1)}$ specified for $\Delta d=70 \mathrm{~mm}$, wave focusing can be achieved thanks to another phase profile $\phi^{(2)}=\frac{2 \pi}{\lambda}\left(\sqrt{x_{0}^{2}+\left(y-y_{0}\right)^{2}}-x_{0}\right)$, where $x_{0}$ and $y_{0}$ are the coordinates of the focal point (white circle on Fig. 7-5(a); therein $x_{0}=100 \mathrm{~mm}$ and $y_{0}=50 \mathrm{~mm}$ ). Numerical results shown in Figs. 7-5(a) and 7-5(b) confirm that each of these profiles achieve its intended functionality predicted analytically. Now, by combining the two phase profiles into a single new transfer function $H=C\left(e^{i \phi^{(1)}}+e^{i \phi^{(2)}}-1\right)$, wave focusing and steering can be achieved simultaneously in order to, say, form two images, one close and one distant, of one source. Figures 7-5(c) and 7-5(d) demonstrate the simulated and experimentally measured wave fields with the new transfer function. It is found that the incident wave can be focused and steered, simultaneously, without any fading. 

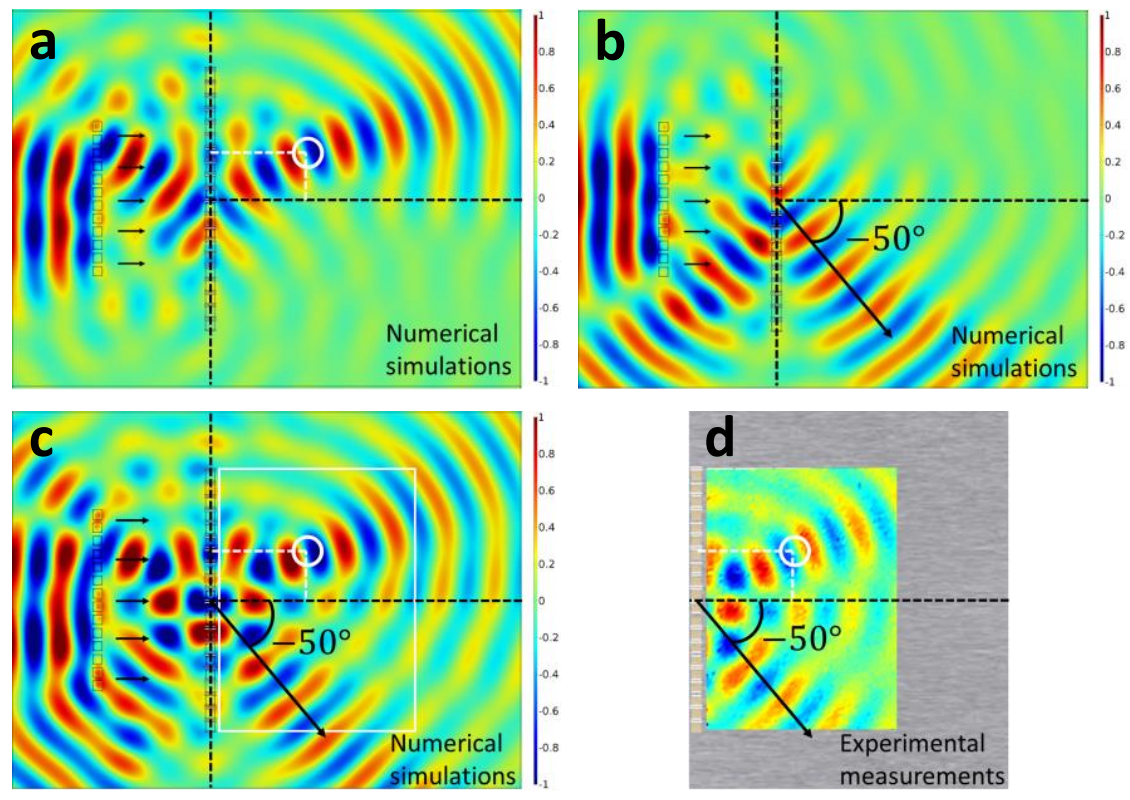

Figure 7-5 Simultaneous focusing and steering. (a,b) Simulated normalized out-of-plane displacement fields manipulated by the metasurface encoded with single phase profile functions: (a) Focusing elastic rays at $x_{0}=100 \mathrm{~mm}, y_{0}=50 \mathrm{~mm}$; (b) Steering elastic rays to $-50^{\circ}$. (c,d) Numerically simulated and experimentally measured out-of-plane velocity fields manipulated by the metasurface encoded with the superposition of the focusing and steering transfer functions of (a) and (b). Wave focusing and steering are then achieved simultaneously: the plot of (c) is identical to the superposition of the two plots (a) and (b).

Here, the extra elastic energy generated is extracted from the electric infrastructure. Programmed electrical and elastic power exchange can be utilized in other cases of elastic wave control as well. By leveraging other transfer functions that channel more electrical energy, the convergent field can be amplified so as to enhance the intensity of the image. First, with unitary transmittance, consider the same focusing phase profile as before. The image distance to the metasurface is given by parameter $x_{0}$ and is changed from 60 to 120 
mm on Figs. 7-6(a) and (b), respectively, thus illustrating how the image position can be tuned. By defining a new transfer function $H=\gamma C e^{i \phi}-C$, the wave transmittance and image intensity will become controllable. For example, in Figs. 7-6(c) and (d), we select $\gamma$ to be 5 and 10, respectively, to enhance the image by 5 and 10 times the original one shown in Fig. 7-6(b). The normalized out-of-plane displacement field shown in the two figures demonstrate the desired enhancement. In Fig. 7-6(e), we extract the magnitude of the normalized out-of-plane displacement field at $x=120 \mathrm{~mm}$ (imaging spot). It is seen then that the image intensity is indeed increased nearly to the exact prescribed value.

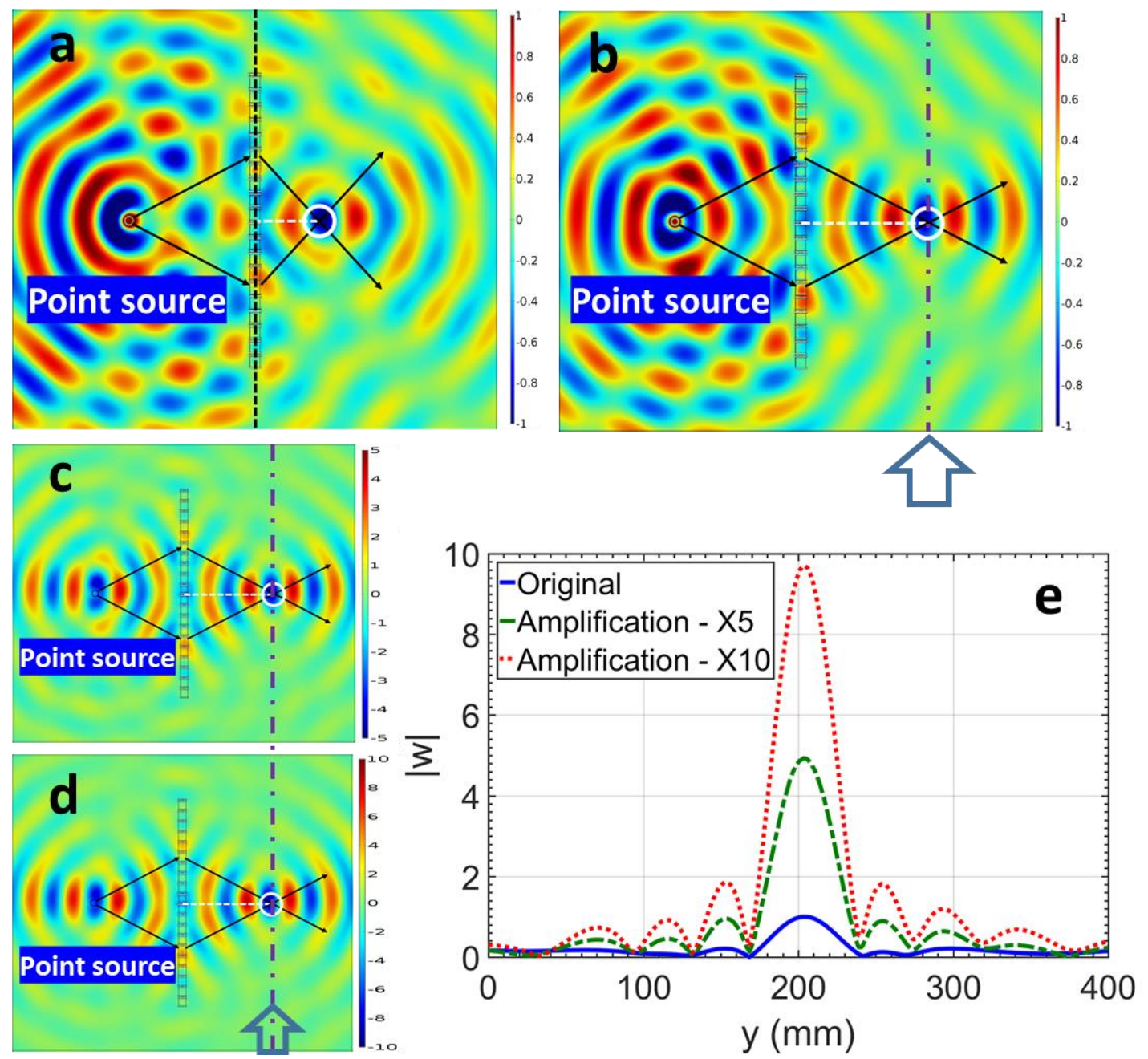


Figure 7-6 Programable metasurface for enhanced imaging of a point source. (a-d) Normalized displacement wave field of the point imaging: $(a, b)$ at different locations (60 and $120 \mathrm{~mm})$ without amplifications. $(\mathrm{c}, \mathrm{d})$ at the same location $(120 \mathrm{~mm})$ with different amplification ratios being 5 and 10. (e) The magnitude of the normalized out-of-plane displacement field at $x=120 \mathrm{~mm}$ with different amplification ratios

\subsubsection{Non-reciprocal wave propagation}

As a third demonstration of the extreme functionalities that the proposed metasurface can achieve, a thin one-way transmitter of flexural waves is realized for the first time. As a matter of fact, the transfer function, being dependent on the wave vector, can be designed to break spatial symmetry and reciprocity. For instance, the metasurface can be programmed to block waves incident from the left while transmitting, or even amplifying, waves incident from the right. Other techniques for breaking reciprocity make use of socalled "dynamic materials" where the constitutive properties need to be constantly changing with time across a depth of multiple wavelengths throughout the bulk of the host medium [108,109]. In comparison, the suggested metasurface appears as an appealing thin, broadband and reprogrammable substitute. Going into specifics, the transfer function is taken to be of the form $H=-\frac{1}{\kappa_{a} \kappa_{s} A \sin \left(k_{b} \delta_{d}\right)}$ so that the transmitted wave vanishes completely $\left(w_{t}=0\right)$ by destructive interference for right-going waves. However, for leftgoing waves, interference is constructive and the transmitted wave will be $w_{t}=2 w_{0} e^{-i k x}$ with an amplitude twice that of the incident wave. Experimental and numerical tests 
confirm the predicted one-way transmission phenomenon and show good agreement [see Figs. 7-7(a) and 7-7(b)].
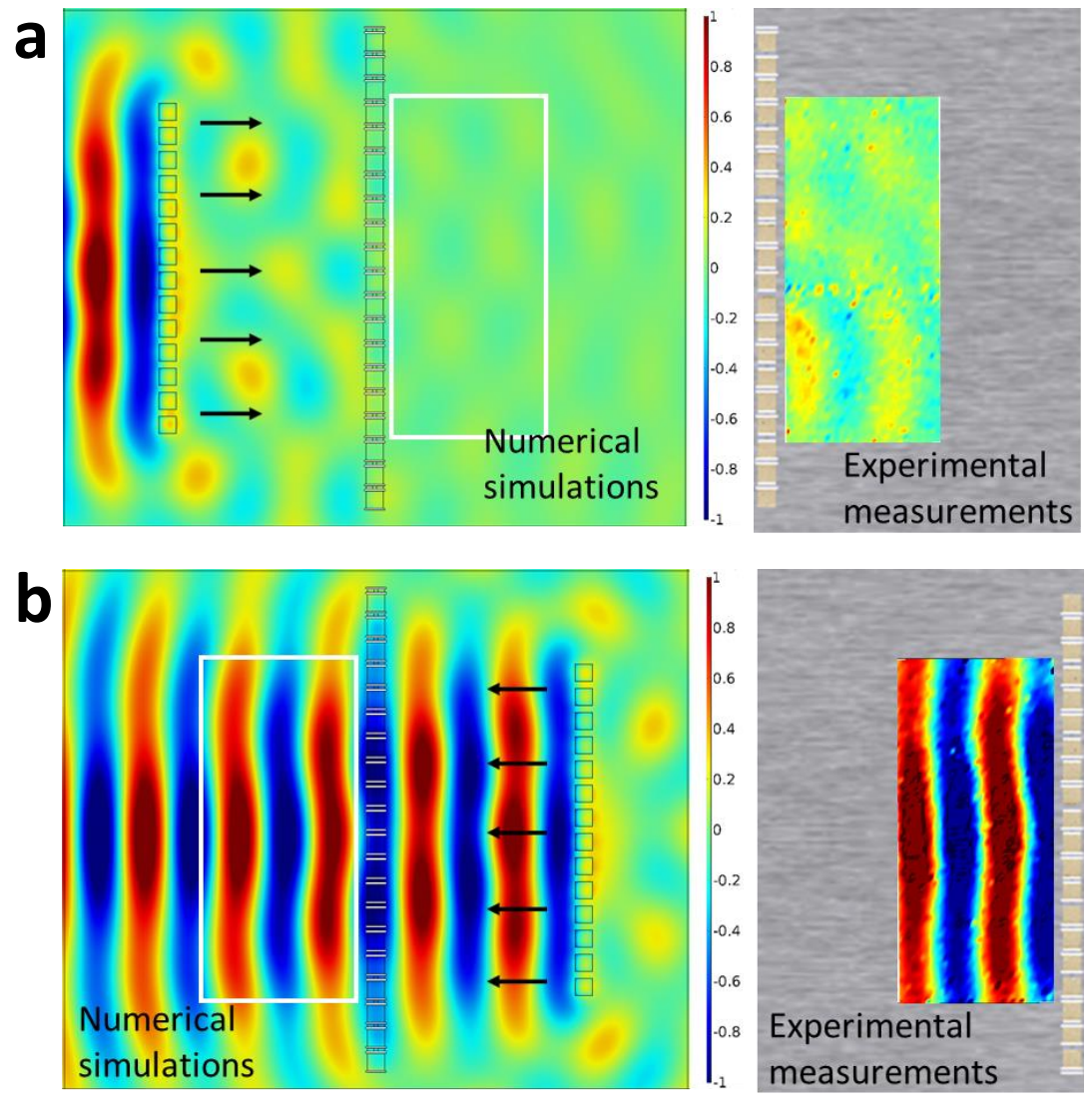

Figure 7-7 One-way wave transmission. (a) Numerically simulated and experimentally measured out-of-plane velocity fields for right-going incident waves. Here, the incident wave is nearly totally blocked. (b) Numerically simulated and experimentally measured out-of-plane velocity fields for left-going incident waves. Here, the incident wave is amplidied to two times its original amplitude 


\subsubsection{Skin cloaking of voids}

Among the array of applications made possible by the proposed metasurface, the realization of cloaking devices is of particular interest. Cloaking refers to strategies by which scattering by a concealed object is suppressed so that its detection by probing waves becomes impossible. Cloaks are usually passive and designed according to one version or another of the transformation method thus requiring the realization of a gradient of materials properties across a given distance equal to the thickness of the cloak [110-112]. Although said thickness can be arbitrarily small in theory, in practice, the thickness of the cloak turns out to be comparable if not larger than the diameter of the cloaked object. In contrast, the present metasurface permits the design of ultrathin cloaks, also referred to as "skin cloaks" [113]. Skin cloaking is demonstrated numerically hereafter in three configurations [see Figs. 7-8(a) - 7-8(c)]. In each configuration, a polygonal portion of the host plate is voided then concealed by a skin cloak. Scattering by the voids is approximated to a good degree of accuracy by reflection occurring on the sides of the voided polygons facing the incident wave. As shown in the left column figures, each boundary common to both the voided polygons and to the plate and facing the incident wave (red lines) will host a metasurface that will suppress reflections (yellow lines) with coherent waves (green lines). As for the metasurfaces occupying the opposite sides, they will generate a coherent field (green lines) equal to the incident one with a phase delay equal to that the incident field would have acquired had it travelled through the voided region. Thus, the metasurfaces occupying opposite sides need to communicate on a cellular level. Through a "feedforward" control loop similar to the one previously used in the steering demonstration, a transfer function which can totally cancel reflected waves while 
generating the appropriate coherent transmitted waves described above can be designed. Results of the numerical simulations are shown on Figs. 7-8(a)-7-8(c). The normalized out-of-plane displacement fields are plotted for right-going incident waves once in the absence of the voided regions and once in their presence, concealed by the proposed skin cloak, at $10 \mathrm{kHz}$. It can be seen that, in each configuration, fields amplitudes and phases are almost identical with and without the voids thus making the voided regions effectively invisible. The presented cloaking configurations show that incidence can be normal [see Fig. 7-8(a)] or oblique (e.g., at $45^{\circ}$, Fig. 7-8(b)). Further, the cloaked region can occupy the boundary of the plate. The situation depicted in Fig. 7-8(c), illustrates a skin carpet cloak. The major difference here is that the cloak needs to compensate for the reflections along the boundary of the plate that would have occurred in the absence of the voids. Here too, Figure 7-8(c) shows that the displacement fields in the presence and absence of the voids are almost identical in terms of amplitude and phase. Note that in cloaking applications, the metasurface is highly efficient energetically since all energy radiated from the metasurface is exploited. Although the invisibility cloaks designed here based on the programmable metasurface are not perfect since, for instance, prior knowledge of the incidence angle is required, the approach remains highly appealing given its simplicity and the extremely small thickness of the designed cloaks. 
a

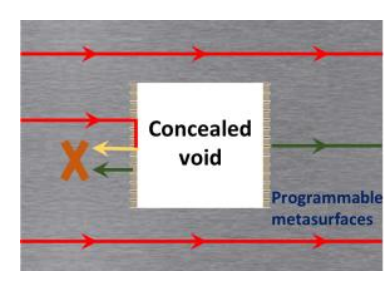

b

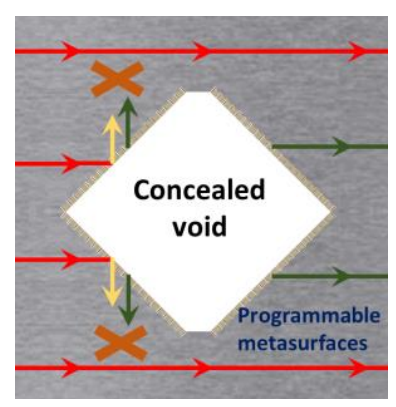

C

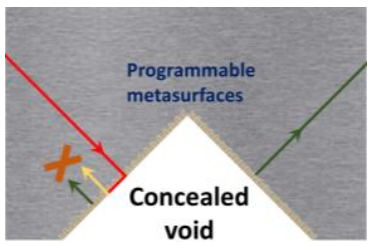

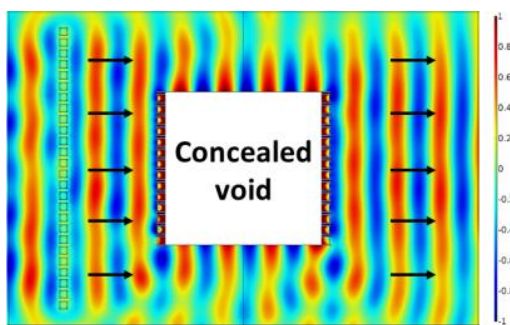
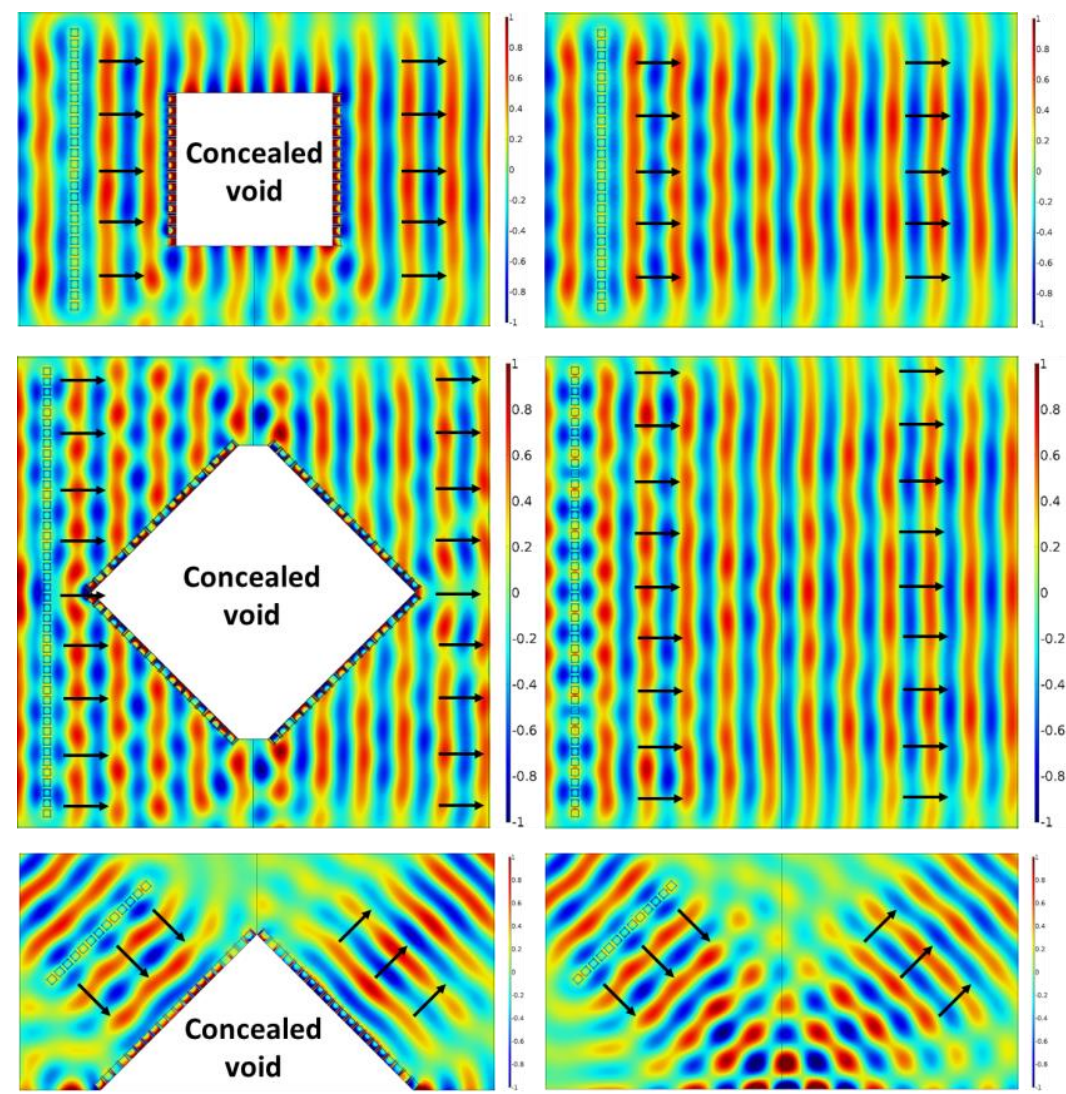

Figure 7-8 Skin cloaking of polygonal voids. (a) Numerical demonstration of skin cloaking of a rectangular void in a plate under normal incidence with the programable metasurface. Red, yellow and green arrowed lines represent incident, reflected and coherent rays, respectively. The perfect destructive wave interference between reflected and coherent waves makes voided boundary facing incident waves reflectionless and effectively invisible. The coherent rays generated on the right metasurface voided boundary makes the voided region invisible to transmitted waves. Simulated normalized out-of-plane displacement fields are almost identical between the plate with a void together with the metasurface and without the void. (b) Numerical demonstration of skin cloaking of a rectangular void in a plate under oblique incidence with the programable metasurface. (c) Numerical demonstration of skin carpet cloaking of a triangular void on a plate boundary with the programable metasurface 


\subsubsection{Broadband operability}

While operating at a subwavelength scale, the metasurface does not suffer from any frequency limitations. Note however that the programmed transfer functions are themselves frequency-dependent since the electromechanical coupling coefficient as well as the wavenumber vary with frequency (Eq. (7.10)). In particular, Figure 7-9(a) shows the numerically determined profile of the amplification ratio $C / A$ of the transfer function with respect to frequency (blue solid curve). Then, it can be seen that larger amplification ratios are required at lower frequencies than at higher ones, mainly because the electromechanical coupling coefficient $\kappa_{s} \kappa_{a}$ and wavenumber $k_{b}$ are much smaller at lower frequencies than at higher frequencies. In practice, the dependency of the amplification ratio on frequency [see Fig. 7-9(a)] can be accurately compensated for by a fourth order transfer function (green dashed curve) easily programmable into digital controllers so that the phase control of the programmable metasurface becomes frequency-independent. The wave transmittance and phase change induced by the metasurface with this fitted forth order transfer function are shown in Figs. 7-9(b) and 7-9(c), where the phase $\phi$ is prescribed as $\pi$. It can be seen that the phase jump strictly follows the prescribed values whereas transmittance present some limited fluctuations that will not significantly affect the performance of the metasurface. As an example, ray steering is demonstrated in Figs. 79(d) and 7-9(e) at two different frequencies of 5 and $15 \mathrm{kHz}$. Therein, the refraction angle is changed due to the dependency of the wavelength on frequency. 

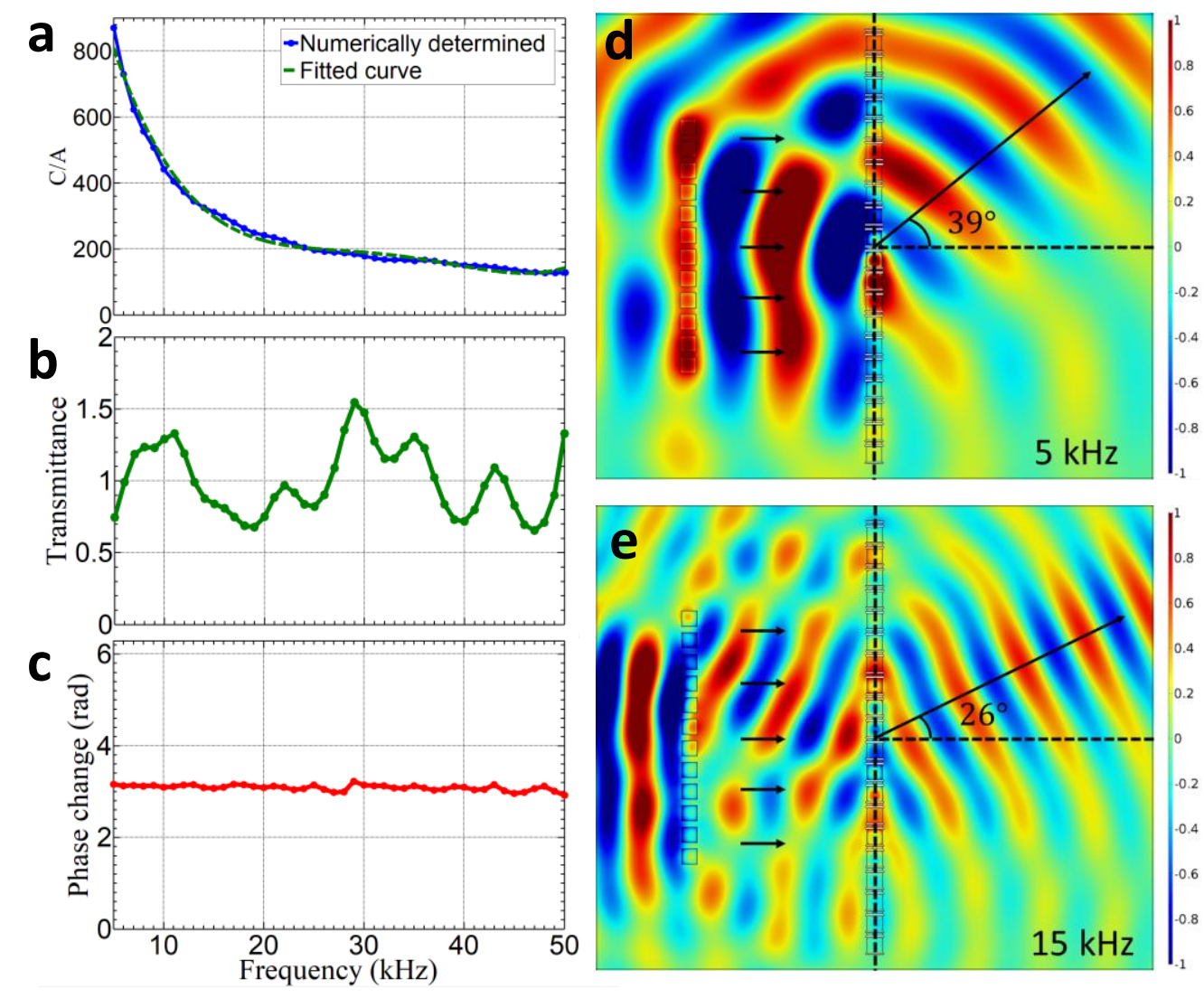

Figure 7-9 Broadband operability of the programmable metasurface. (a) Amplification ratio of the transfer function, C/A, determined numerically at different frequencies and its numerically fitted forth order curve. (b) Wave transmittance induced by the metasurface with the fitted fourth order transfer function. (c) Wave phase change induced by the metasurface with the fitted fourth order transfer function. (d,e) Simulated normalized outof-plane displacement fields manipulated by the metasurface: (d) at $5 \mathrm{kHz}$; (e) at $15 \mathrm{kHz}$

\subsection{Conclusions}

The suggested programmable elastic metasurface with self-sensing-and-actuating units provides an appealing platform for real-time, multifunctional and asymmetric trajectory control of elastic waves. By simply programming electric circuits, we were able, for the 
first time, to control in real time the ray paths of transmitted signals, be them rectilinear or curvilinear. In addition, by selecting particular transfer functions, the design can support one-way non-reciprocal wave blocking as well as cloaking functionalities. These are but a few examples of the range of functions that the programmable metasurface can achieve. The presented approach can be extended to different time and length scales and to different wave systems. The immediate applications of the concept we foresee are related to stealth technology, active noise control and the realization of ideal experimental platforms with isolated or otherwise controlled environments. 


\section{Chapter 8 Conclusions}

The dissertation introduces some active, adaptive, hybrid, and programmable metamaterials and metasurfaces for real time, reconfigurable, and multifunctional elastic wave control. By integrating analytical, numerical and experimental approaches, the designs focus on demonstrating tunable and super-broadband elastic wave band gap control and trajectory manipulations. In particular, an active elastic metamaterial with negative capacitance piezoelectric shunting circuits is presented, where the location and the width of the local resonant band gap can be effectively tuned. Followed by this concept, we experimentally demonstrate the tunable band gap with an active metamaterial tube. Our experimental results demonstrate that the tunable bandgap capacity, as high as $45 \%$, is physically realized by using both hardening and softening shunted circuits. However, the controlled band gap is still narrow in real time, and cannot be used to mitigate broadband incident loadings. Therefore, by using the frequency-dependent stiffness concept, we design an adaptive metamaterial beam with hybrid shunting circuits to realize super broadband Lamb wave band gaps. The adaptive mechanical mechanism within the tunable resonator can produce high-pass and low-pass wave filtering capabilities for the zerothorder anti-symmetric Lamb wave modes. Instead of tuning the stiffness of the resonators, we propose a hybrid metamaterial that modulating the stiffness in the host medium. Our numerical results show that the flexural wave propagation can be switched between "ON/OFF" states with different stiffness. The programmed flexural wave manipulations, broadband negative refraction and waveguiding are also demonstrated with threedimensional multi-physical numerical simulations. For elastic wave trajectory control, we

first propose a transformation method to derive the material properties of a flexural 
waveguide, and implement its functionality based on an active elastic metamaterials. The numerically demonstrated flexural waveguide can steer an elastic wave beam to different directions at broadband frequencies. Finally, we introduce an ultrathin programmable elastic metasurface design with self-sensing-and-actuating units, where real time ray steering of flexural waves in a host plate is achieved. We also experimentally demonstrate the simultaneously multifunctional control and one-way non-reciprocal blocking of waves. Skin cloaking of voids and operability across broad wave frequency ranges are tested numerically. The designs suggested in the dissertation could immediately open new possibilities in elastic wave control devices including, but not limited to structural health monitoring, stealth technology, active noise control, as well as medical instrumentation and imaging.

The research of active elastic metamaterials is highly multidisciplinary, bridging mechanical and electrical engineering and information science. By properly combining advances in each area, the active elastic metamaterial could produce new functionalities. The active approaches in metamaterial engineering are extremely necessary in some of scenarios, where passive methods cannot be used or extremely costly, i.e. to break time reversal symmetry with dynamic materials and to achieve parity-time symmetric medium with both loss and gain components. With the developments in classical and quantum wave theories and fabrications in soft and flexible materials and structures, active elastic metamaterials will have a brilliant future and attract more research attentions. 


\section{Reference}

[1] Brillouin L. Wave propagation in periodic structures. Dover Publications, second edition, 1953.

[2] Hussein M I, Leamy M J and Ruzzene M, Dynamics of phononic materials and structures: Historical origins, recent progress and future outlook. Applied Mechanics Reviews, 2014, 66: 040802.

[3] Ma G and Sheng P, Acoustic metamaterials: From local resonances to broad horizons. Science Advances, 2016, 2: e1501595.

[4] Cummer S A, Christensen J and Alù A, Controlling sound with acoustic metamaterials. 2016, Nature Reviews Materials, 1: 16001.

[5] Joannopoulos J D, Villeneuve P R, and Fan S, Photonic crystals: putting a new twist on light. Nature, 1997, 386: 143-149.

[6] Montero de Espinosa F R, Jimenez E and Torres M, Ultrasonic band gap in a periodic two-dimensional composite. Physical Review Letters, 1998, 80: 12081211.

[7] Haberman M and Guild M, Acoustic metamaterials. Physics Today, 2016, 69: 4248.

[8] Liu Z Y, Zhang X, Mao Y, Zhu Y Y, Chan C T, and Sheng P, Locally resonant sonic materials. Science, 2000, 289: 1734.

[9] Huang H H, Sun C T and Huang G L, On the negative effective mass density in acoustic metamaterials. International Journal of Engineering Science, 2009, 47: 610.

[10] Yao S S, Zhou X M and Hu G K, Experimental study on negative effective mass in 
a 1D mass-spring system. New Journal of Physics, 2008,10: 043020.

[11] Yu D L, Liu Y Z, Wang G, Zhao H G and Qiu J, Flexural vibration band gaps in Timoshenko beams with locally resonant structures. Journal of Applied Physics, 2006, 100: 124901 .

[12] Liu X N, Hu G K, Huang G L and Sun C T, An elastic metamaterial with simultaneously negative mass density and bulk modulus. Applied Physics Letters, 2011, 98: 251907

[13] Yang Z Y, Mei J, Yang M, Chan N H, and Sheng P, Membrane-type acoustic metamaterial with negative dynamic mass. Physical Review Letters, 2008, 101(20): 204301.

[14] Huang G L and Sun C T, Band gaps in a multiresonator acoustic metamaterial. ASME Journal of Vibration and Acoustics, 2010, 132: 031003.

[15] Zhu R, Huang G L, Huang $\mathrm{H} \mathrm{H}$ and Sun C T, Microstructure design and experimental validation of elastic metamaterial plates with anisotropic mass density. Physical Review B, 2012, 86:144307.

[16] Ding Y, Liu Z Y, Qiu C Y and Shi J, Metamaterial with simultaneously negative bulk modulus and mass density. Physical Review Letters, 2007, 99: 093904.

[17] Zhu R, Liu X N, Hu G K, Sun C T and Huang G L, A chiral elastic metamaterial beam for broadband vibration suppression. Journal of Sound and Vibration, 2014, 333: 2759-2773.

[18] Assouar M B, Senesi M, Oudich M, Ruzzene M, and Hou Z, Broadband plate-type acoustic metamaterial for low-frequency sound attenuation. Applied Physics Letters, 2012, 101(17): 173505-173505. 
[19] Zhou X L and Chen C Q, Tuning the locally resonant phononic band structures of two-dimensional periodic electroactive composites. Physica B: Condensed Matter, 2013, 431: 23-31.

[20] Baz A, The structure of an active acoustic metamaterial with tunable effective density. New Journal of Physics, 2009, 11: 123010.

[21] $\mathrm{Xu} \mathrm{Z}$ and Wu F, Elastic band gaps of magnetorheological elastomer vibration isolators. Journal of Intelligent Material Systems and Structures, 2014, 7(26): 858864.

[22] Yeh J Y, Control analysis of the tunable phononic crystal with electrorheological material. Physica B: Condensed Matter, 2007, 400: 137-144.

[23] Fang N, Xi D, Xu J, Ambati M, Srituravanich W, Sun C, and Zhang X, Ultrasonic metamaterials with negative modulus. Nature Material, 2006, 5(6): 452-456.

[24] Wu Y, Lai Y and Zhang Z Q, Elastic metamaterials with simultaneously negative shear modulus and mass density. Physical Review Letters, 2011, 107: 105506.

[25] Mei J, Ma G C, Yang M, Yang Z, Wen W J and Sheng P, Dark acoustic metamaterials as super absorbers for low-frequency sound. Nature Communication, 2012, 3: 756.

[26] Chen Y Y, Huang G L, Zhou X M, Hu G K and Sun C T, Analytical coupled vibroacoustic modeling of membrane-type acoustic metamaterials: Membrane model. Journal of the Acoustical Society of America, 2014, 136: 969-979.

[27] Chen Y Y, Huang G L, Zhou X M, Hu G K and Sun C T, Analytical coupled vibroacoustic modeling of membrane-type acoustic metamaterials: Plate model. Journal of the Acoustical Society of America, 2014, 136: 2926-2934. 
[28] Zhou X M, Liu X N, Hu G K and Huang G L, Micromechanics of Elastic Metamaterials. Handbook of Micromechanics and Nanomechanics, 2013, 29-72, Pan Stanford Publishing, ISBN 978-981-4411-23-3.

[29] Zhu R, Liu X N, Hu G K, Sun C T and Huang G L, Negative refraction of elastic waves at the deep subwavelength scale in a single-phase metamaterial. Nature Communication, 2014, 5: 5510.

[30] Farhat M, Enoch S, Guenneau S and Movchan A B, Broadband cylindrical acoustic cloak for linear surface waves in a fluid. Physical Review Letters, 2008, 101: 134501

[31] Pai P F, Metamaterial-based broadband elastic wave absorber. Journal of Intelligent Material Systems and Structures, 2010, 21: 517.

[32] Liu A P, Zhu R, Huang G L and Hu G K, Super-resolution imaging by resonant tunneling in anisotropic acoustic metamterials. Journal of the Acoustic Society of America, 2012, 132: 2800.

[33] Yan X, Zhu R, Huang G L and Yuan F G, Focusing guided waves using surface bonded elastic metamaterials. Applied Physics Letters, 2013, 103: 121901.

[34] Zhang S, Xia C G and Fang N, Broadband acoustic cloak for ultrasound waves. Physical Review Letters, 2011,106: 024301.

[35] Brûlé S, Javelaud E, Enoch S and Guenneau S, Experiments on seismic metamaterials: molding surface waves. Physical Review Letters, 2014, 112: 133901.

[36] Krodel S, Thomé N and Daraio C, Wide band-gap seismic metastructures. Extreme Mechanics Letters, 2015, 4: 111-117. 
[37] Wu T T, Huang Z G, Tsai T C and Wu T C, Evidence of complete band gap and resonances in a plate with periodic stubbed surface. Applied Physics Letters, 2008, 93: 111902 .

[38] Milton G W and Nicorovici N A P, On the cloaking effects associated with anomalous localized resonance. Proceedings of the Royal Society of London A, 2006, 462: 3027-3059.

[39] Wang P, Casadei F, Shan S, Weaver J C and Bertoldi K, Harnessing buckling to design tunable locally resonant acoustic metamaterials. Physical Review Letters, 2014, 113: 014301 .

[40] Chen Y Y, Barnhart M V, Chen J K, Hu G K, Sun C T, Huang G L, Dissipative elastic metamaterials for broadband wave mitigation at subwavelength scale, Composite Structures, 2016, 136:358-371.

[41] Lee H, Oh J H, Seung H M, Cho S H and Kim Y Y, Extreme stiffness hyperbolic elastic metamaterial for total transmission subwavelength imaging. Scientific Reports, 2016, 6:24026.

[42] Chen Y, Huang G L and Sun C T, Band gap control in an active elastic metamaterial with negative capacitance piezoelectric shunting. ASME Journal of Vibration and Acoustics, 2014, 136: 061008.

[43] Zhu R, Chen Y Y, Barnhart M V, Hu G K, C T Sun and Huang G L, Experimental study of an adaptive elastic metamaterial controlled by electric circuits. Applied Physics Letters, 2016, 108: 011905.

[44] Chen Y Y, Hu G K and Huang G L, An adaptive metamaterial beam with hybrid shunting circuits for extremely broadband control of flexural waves. Smart 
Materials and Structures, 2016, 25 (10):105036.

[45] Chen Y Y and Huang G L, Active elastic metamaterials for subwavelength wave propagation control. Acta Mechanica Sinica, 2015, 31(3): 349-363.

[46] Chen Y Y, Hu J and Huang G L, A design of active elastic metamaterials for control of flexural waves using the transformation method, Journal of Intelligent Material Systems and Structures, 2016, 27 (10):1337-1347.

[47] Chen Y Y, Zhu R, Barnhart M V and Huang G L, Enhanced flexural wave sensing by adaptive gradient-index metamaterials. Scientific Reports, 2016, 6:35048.

[48] Chen Y Y, Hu G K and Huang G L, A hybrid elastic metamaterial with negative mass density and tunable bending stiffness, Journal of the Mechanics and Physics of Solids, 2017, 105:179-198.

[49] Forward R L, Electronic damping of vibrations in optical structures. Journal of Applied Optics, 1979,18(5): 690-697.

[50] Hagood N W and Flotow A V, Damping of structural vibrations with piezoelectric materials and passive electrical networks. Journal of Sound and Vibration, 1991, 146(2): 243-268.

[51] Mokry P, Fukada E, and Yamamoto K, Sound absorbing system as an application of the active elasticity control technique. Journal of Applied Physics, 2003, 94(11): $7356-7362$.

[52] Wang G, Wang J, Chen S and Wen J, Vibration attenuations induced by periodic arrays of piezoelectric patches connected by enhanced resonant shunting circuits. Smart Materials and Structures, 2011, 20: 125019.

[53] Chen S B, Wen J H, Yu D L, Wang G and Wen X S, Band Gap Control of Phononic 
Beam With Negative Capacitance Piezoelectric Shunt. Chinese Physics B, 2011, 20(1): 014301.

[54] Collet M, Ouisse M and Ichchou M, Structural energy flow optimization through adaptive shunted piezoelectric metacomposites. Journal of Intelligent Material Systems and Structures, 2012, 23(15): 1661-1677.

[55] Airoldi L and Ruzzene M, Design of tunable acoustic metamaterials through periodic arrays of resonant shunted piezos. New Journal of Physics, 2011, 13(11): 113010.

[56] Casadei F, Delpero T, Bergamini A, Ermanni P and Ruzzene M, Piezoelectric resonator arrays for tunable acoustic waveguides and metamaterials. Journal of Applied Physics. 2012, 112: 064902.

[57] Bergamini A, Delpero T, De Simoni L, Di Lillo L, Ruzzene M and Ermanni P, Phononic crystal with adaptive connectivity. Advanced Materials, 2013, 2: 13431347.

[58] Collet M, Ouisse M and Tateo F, Adaptive metacomposites for vibroacoustic control applications. IEEE Sensors Journal, 2014, 14(7): 2145-2152.

[59] Wu S-Y, Multiple PZT transducers implemented with multiple mode piezoelectric shunting for passive vibration damping. SPIE Conference on Passive Damping, Newport Beach, 1999, 3672:112-122.

[60] Dell'Isola F, Maurini C and Porfiri M, Passive damping of beam vibrations through distributed electric networks and piezoelectric transducers: prototype design and experimental validation. Smart Materials and Structures, 2004, 13: 299-308.

[61] Airoldi L and Ruzzene M, Wave propagation control in beams through periodic 
multi-branch shunts. Journal of Intelligent Material Systems and Structures, 2011, 22: $1567-1579$.

[62] Behrens S, Fleming A J and Moheimani S O R, A broadband controller for shunt piezoelectric damping of structural vibration. Smart Materials and Structures, 2003, 12: 18-28.

[63] Park C H and Baz A, Vibration control of beams with negative capacitive shunting of interdigital electrode piezoceramics. Journal of Sound and Vibration, 2005, 11: $331-346$.

[64] Neubauer M, Oleskiewicz R, Popp K and Krzyzynski T, Optimization of damping and absorbing performance of shunted piezo elements utilizing negative capacitance. Journal of Sound and Vibration, 2006, 298: 84-107.

[65] Beck B S, Cunefare K A, Ruzzene M and Collet M, Experimental analysis of a cantilever beam with a shunted piezoelectric periodic array. Journal of Intelligent Material Systems and Structures, 2011, 22: 1177-1187.

[66] Tateo F, Collet M, Ouisse M, Ichchou M, Cunefare K and Abbe P, Experimental characterization of a bidimensional array of negative capacitance piezo-patches for vibroacoustic control. Journal of Intelligent Material Systems and Structures, 2014, 26: $952-964$.

[67] de Marneffe B and Preumont A, Vibration damping with negative capacitance shunts: theory and experiment. Smart Materials and Structures, 2008, 17: 035015.

[68] Nečásek J, Václavík J and Marton P, Digital synthetic impedance for application in vibration damping. Review of Scientific Instruments, 2016, 87: 024704.

[69] Fleming A J, Behrens S and Moheimani S O R, Synthetic impedance for 
implementation of piezoelectric shunt-damping circuits. Electronics Letters, 2000, 36(18): $1525-1526$.

[70] Fleming A J and Moheimani S O R, Adaptive piezoelectric shunt damping. Smart Materials and Structures, 2003, 12: 36-48.

[71] Matten G, Collet M, Cogan S and Sadoulet-Reboul E, Synthetic impedance for adaptive piezoelectric metacomposite. Procedia Technology, 2014, 15: 84-89.

[72] Wang G, Cheng J, Chen J and He Y, Multi-resonant piezoelectric shunting induced by digital controllers for subwavelength elastic wave attenuation in smart metamaterial. Smart Materials and Structures, 2017, 26:025031.

[73] De Marchi L, Marzani A, Caporale S and Speciale N, Ultrasonic guided waves characterization with warped frequency transforms. IEEE Transactions on Ultrasonics, Ferroelectrics and Frequency Control, 2009, 56: 2232-2240.

[74] Bergamini A E, Zündel M, Flores Parra E A, Delpero T, Delpero T, Ruzzene M and Ermanni P. Hybrid dispersive media with controllable wave propagation: a new take on smart materials. Journal of Applied Physics, 2015, 118:154310.

[75] Wang G and Chen S B, Large low-frequency vibration attenuation induced by arrays of piezoelectric patches shunted with amplifier-resonator feedback circuits. Smart Materials and Structures, 2016, 25:015004.

[76] Cardella D, Celli P and Gonella S, Manipulating waves by distilling frequencies: a tunable shunt-enabled rainbow trap. Smart Materials and Structures, 2016, 25(8):085017.

[77] Yi K, Collet M, Ichchou M and Li L, Flexural waves focusing through shunted piezoelectric patches. Smart Materials and Structures, 2016, 25:075007. 
[78] Ouisse M, Collet M and Scarpa F, A piezo-shunted kirigami auxetic lattice for adaptive elastic wave filtering. Smart Materials and Structures, 2016, 25:115016.

[79] Celli P and Gonella S, Tunable directivity in metamaterials with reconfigurable cell symmetry. Applied Physics Letters, 2015, 106:091905.

[80] Pendry J B, Shurig D and Smith D R, Controlling electromagnetic fields. Science, 2006, 312:1780-1782.

[81] Chen H Y and Chan C T, Acoustic cloaking in three dimensions using acoustic metamaterials. Applied Physics Letters, 2007, 91:183518.

[82] Chen H Y and Chan C T, Acoustic cloaking and transformation acoustics. Journal of Physics D: Applied Physics, 2010, 43:113001.

[83] Milton G W, Briane M and Willis J R, On cloaking for elasticity and physical equations with a transformation invariant form. New Journal of Physics, 2006, 8: 248.

[84] Farhat M, Guenneau S and Enoch S, Ultrabroadband elastic cloaking in thin plates. Physical Review Letters, 2009, 103:024301.

[85] Chang Z, Hu J, Hu G K, Tao R and Wang Y, Controlling elastic waves with isotropic materials. Applied Physics Letters, 2011, 98:121904.

[86] Hu J, Chang Z and Hu G K, Approximate method for controlling solid elastic waves by transformation media. Physical Review B, 2011, 84:201101(R).

[87] Zhou X L and Chen C Q, Tuning the locally resonant phononic band structures of two-dimensional periodic electroactive composites. Physica B: Condensed Matter, 2013, 431:23-31.

[88] Liu B and Shaw M T, Electrorheology of filled silicone elastomers. Journal 
of Rheology, 2001, 45:641-657.

[89] Wei K X, Bai Q, Meng G and Ye L, Vibration characteristics of electrorheological elastomer sandwich beams. Smart Materials and Structures, 2011, 20:055012.

[90] Hsiao H H, Chu C H, and Tsai D P, Fundamentals and applications of metasurfaces. Small Methods, 2017, 1:1600064.

[91] Yu N, Genevet P, Kats M A, Aieta F, Tetienne J P, Capasso F and Gaburro Z, Light propagation with phase discontinuities: generalized laws of reflection and refraction. Science, 2011, 334:333.

[92] Lin D, Fan P, Hasman E and Brongersma M L, Dielectric gradient metasurface optical elements. Science, 2014, 345:298.

[93] Zheng G, Mühlenbernd H, Kenney M, Li G, Zentgraf T and Zhang S, Metasurface holograms reaching 80\% efficiency. Nature Nanotechnology, 2015, 10:308.

[94] Zhang L, Mei S, Huang K and Qiu C, Advances in full control of electromagnetic waves with metasurfaces. Advanced Optical Materials, 2016, 4: 818.

[95] Chen K, Feng Y, Monticone F, Zhao J, Zhu B, Jiang T, Zhang L, Kim Y, Ding X, Zhang S, Alù A and Qiu C W, A reconfigurable active Huygens' metalens. Advanced Materials, 2017, 29:1606422.

[96] Xie Y, Wang W, Chen H, Konneker A, Popa B-I and Cummer S A, Wavefront modulation and subwavelength diffractive acoustics with an acoustic metasurface. Nature Communications, 2014, 5:5553.

[97] Li Y, Jiang X, Li R Q, Liang B, Zou X-Y, Yin L-L and Cheng J-C, Experimental realization of full control of reflected waves with subwavelength acoustic metasurfaces. Physical Review Applied, 2014, 2:064002. 
[98] Mei $\mathrm{J}$ and $\mathrm{Wu} \mathrm{Y,} \mathrm{Controllable} \mathrm{transmission} \mathrm{and} \mathrm{total} \mathrm{reflection} \mathrm{through} \mathrm{an}$ impedance-matched acoustic metasurface. New Journal of Physics, 2014, $16: 123007$.

[99] Memoli G, Caleap M, Asakawa M, Sahoo D R, Drinkwater B W and Subramanian S, Metamaterial bricks and quantization of meta-surfaces. Nature Communications, 2017, 8:14608.

[100] Popa B I, Shinde D, Konneker A and Cummer S A, Active acoustic metamaterials reconfigurable in real time. Physical Review B, 2015, 91:220303.

[101] Zhu H and Semperlotti F, Anomalous refraction of acoustic guided waves in solids with geometrically tapered metasurfaces. Physical Review Letters, 2016, 117:034302.

[102] Su X and Norris A, Focusing, refraction, and asymmetric transmission of elastic waves in solid metamaterials with aligned parallel gaps. Journal of the Acoustical Society of America, 2016, 139:3386.

[103] Liu Y, Liang Z, Liu F, Diba O, Lamb A and Li J, Source illusion devices for flexural Lamb waves using elastic metasurfaces. Physical Review Letters, 2017, 119:034301.

[104] Zhu B O, Zhao J and Feng Y, Active impedance metasurface with full $360^{\circ}$ reflection phase tuning. Scientific Reports, 2013, 3:3059.

[105] Cui T J, Qi M Q, Wan X, Zhao J and Cheng Q, Broadband diffusion of terahertz waves by multi-bit coding metasurfaces. Light: Science \& Applications, 2014, 3:e218.

[106] Liu S and Cui T J, Flexible controls of terahertz waves using coding and 
programmable metasurfaces. IEEE Journal of Selected Topics in Quantum Electronics, 2017, 23:1.

[107] Popa B I and Cummer S A, Non-reciprocal and highly nonlinear active acoustic metamaterials. Nature Communications, 2014, 5:3398.

[108] Nassar H, Xu X C, Norris A N and Huang G L, Modulated phononic crystals: Nonreciprocal wave propagation and Willis materials. Journal of the Mechanics and Physics of Solids, 2017, 101:10.

[109] Nassar H, Chen H, Norris A N, Haberman M R and Huang G L, Non-reciprocal wave propagation in modulated elastic metamaterials. Proceedings of the Royal Society of London A, 2017, 473:20170188.

[110] Pendry J B, Shurig D and Smith D R, Controlling electromagnetic fields. Science, 2006, 312:1780.

[111] Chen Y, Zheng M, Liu X, Bi Y, Sun Z, Xiang P, Yang J and Hu G, Broadband solid cloak for underwater acoustics. Physical Review B, 2017, 95:180104.

[112] Farhat M, Guenneau S and Enoch S, Ultrabroadband elastic cloaking in thin plates. Physical Review Letters, 2009, 103:024301.

[113] Ni X, Wong Z J, Mrejen M, Wang Y and Zhang X, An ultrathin invisibility skin cloak for visible light. Science, 2015, 349:1310. 


\section{VITA}

Yangyang Chen was born in 1987, and obtained his Bachelor degree in Mechanical Engineering and Master degree in Engineering Mechanics both from Ningbo University, Ningbo, Zhejiang, China. He started his Ph. D. study in the Department of Systems Engineering at the University of Arkansas at Little Rock since August 2012, and then transferred to the Department of Mechanical and Aerospace Engineering at the University of Missouri-Columbia in August 2014 to continue his Ph. D. study. 